Universidad Nacional de la Plata

Facultad de Ciencias Exactas

Departamento de Física

Trabajo de Tesis Doctoral

\title{
Búsqueda de Supersimetría en eventos con un fotón, jets y energía faltante con el detector ATLAS
}

Francisco Alonso

Dirección:

Prof. Dra. María Teresa Dova 



\title{
Búsqueda de Supersimetría en eventos con un fotón, jets y energía faltante con el detector ATLAS
}

\author{
Francisco Alonso
}

\begin{abstract}
Resumen
Supersimetría (SUSY) es una de las teorías con mayor motivación teórica para física más allá del Modelo Estándar, proporcionando un marco para la unificación de la física de partículas y la gravedad, gobernada por la escala de energía de Planck. Dado que ninguna de las partículas supersimétricas predichas ha sido observada, SUSY debe ser una simetría rota en la naturaleza. La fenomenología de SUSY está ampliamente determinada por el mecanismo de rompimiento de la supersimetría. Los modelos GGM en los que el rompimiento está mediado por los campos de gauge usuales del Modelo Estándar brindan un escenario propicio para la búsqueda de SUSY en el LHC, con espectros de masas y decaimientos característicos. En esta tesis se presenta la primera búsqueda de nueva física en un estado final con un fotón energético, jets y gran cantidad de energía faltante en colisiones protón-protón a una energía de centro de masa de $8 \mathrm{TeV}$ en el LHC. El análisis fue realizado utilizando todos los datos recolectados por el detector ATLAS durante el año 2012, que corresponden a una luminosidad total integrada de $20.3 \mathrm{fb}^{-1}$. No se observó un exceso de eventos por sobre las predicciones del Modelo Estándar, por lo cual se estableció un límite superior a 95\% CL al número de eventos provenientes de nueva física para este estado final. Adicionalmente, los resultados fueron interpretados en el contexto de un modelo de GGM SUSY considerando un neutralino NLSP mezcla bino-higgsino, excluyendo la producción de gluinos con masas de hasta $1.25 \mathrm{TeV}$, resultando en los límites más estrictos al presente.
\end{abstract}





\title{
Search for Supersymmetry in events with a photon, jets and missing energy with the ATLAS detector
}

\author{
Francisco Alonso
}

\begin{abstract}
Supersymmetry (SUSY) is one of the most motivated theories for physics beyond the Standard Model, giving a framework to the unification of the particle physics and gravity, which is governed by the Plank energy scale. As none of the predicted supersymmetric particles has been discovered, SUSY must be a broken symmetry in nature. SUSY phenomenology is hardly determined by the SUSY breaking mechanism. GGM models in which the SUSY breaking is mediated by the usual gauge fields of the Standard Model provide a suitable scenario for SUSY searches at the LHC, with very characteristic mass spectrum and decays. This thesis presents the first search of new physics with one energetic photon, jets and high missing energy in the final state in proton-proton collisions at a centre-of mass energy of $8 \mathrm{TeV}$. The analysis was realized with all the data collected by the ATLAS detector during 2012, corresponding to a total integrated luminosity of $20.3 \mathrm{fb}^{-1}$. No excess over the Standard Model predictions was observed, so an upper limit in the number of new physics events was established at $95 \% \mathrm{CL}$ for this final state. Additionally, the results were also interpreted in the context of a GGM SUSY model considering a bino-higgsino neutralino NLSP admixture, excluding the production of gluinos with masses up to $1.25 \mathrm{TeV}$, giving the most strict limits at the moment.
\end{abstract}





\section{ÍNDICE GENERAL}

$\begin{array}{ll}\text { Introducción } & 11\end{array}$

$\begin{array}{lll}\text { PARTE I MOTIVACIÓN TEÓRICA } & \mathbf{1 5}\end{array}$

1 Modelo Estándar y Supersimetría $\quad 17$

1.1 Conceptos básicos del Modelo Estándar . . . . . . . . . . . . . . . . . . . . 17

1.1.1 Las partículas elementales y sus interacciones . . . . . . . . . . . . . . 17

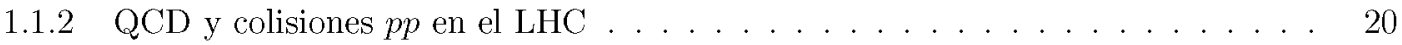

1.1 .3 Física más allá del SM . . . . . . . . . . . . . . . . . . . . . . 22

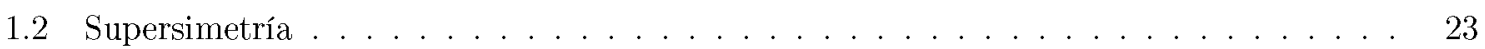

1.2.1 Modelo Estándar Supersimétrico Mínimo . . . . . . . . . . . . . . . 25

1.2 .2 Origen de la ruptura de SUSY. Modelos GMSB . . . . . . . . . . . . . . 31

1.2.3 Producción de partículas supersimétricas . . . . . . . . . . . . . . . . 35

$\begin{array}{lll}\text { PARTE II } & \text { EXPERIMENTO } & 37\end{array}$

$2 \quad$ El LHC y el detector ATLAS

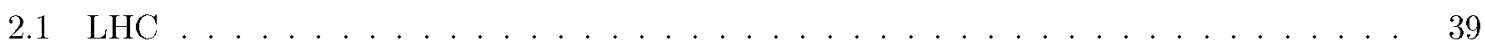

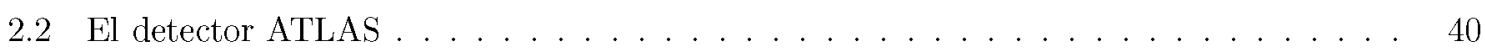

2.2 .1 Sistema de coordenadas . . . . . . . . . . . . . . . . . . . . . . . . 40

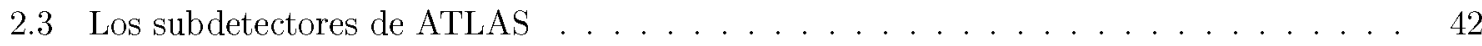

$2.3 .1 \mathrm{El}$ detector interno . . . . . . . . . . . . . . . . . . . . . . 42

2.3 .2 Calorímetros . . . . . . . . . . . . . . . . . . . . . . . . 44

2.3.3 Espectrómetro de muones . . . . . . . . . . . . . . . . . . . . . 45

$2.4 \mathrm{El} \mathrm{sistema} \mathrm{de} \mathrm{trigger} \mathrm{.} \mathrm{.} \mathrm{.} \mathrm{.} \mathrm{.} \mathrm{.} \mathrm{.} \mathrm{.} \mathrm{.} \mathrm{.} \mathrm{.} \mathrm{.} \mathrm{.} \mathrm{.} \mathrm{.} \mathrm{.} \mathrm{.} \mathrm{.} \mathrm{.} \mathrm{.} \mathrm{.} \mathrm{.} \mathrm{.} \mathrm{.} \mathrm{.} 45$

2.5 Modelo computacional y distribución de datos . . . . . . . . . . . . . . . . 47

2.6 Datos de colisiones $p p$ a $\sqrt{s}=8 \mathrm{TeV} \ldots \ldots \ldots \ldots \ldots \ldots \ldots$

3 Reconstrucción e identificación de objetos físicos $\quad 51$

3.1 Reconstrucción de trazas y vértices . . . . . . . . . . . . . . . 51

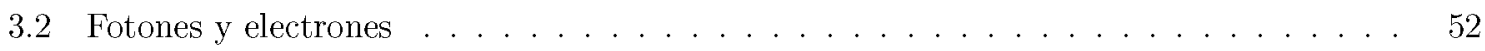

3.2 .1 Reconstrucción . . . . . . . . . . . . . . . . . . . . . 52

3.2 .2 Identificación de fotones . . . . . . . . . . . . . . . . . . 53

3.2 .3 Identificación de electrones . . . . . . . . . . . . . . . . . . . . . 58

3.3 Muones . . . . . . . . . . . . . . . . . . . . . . . . . . 58

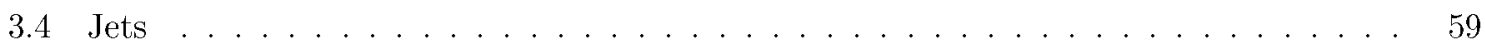

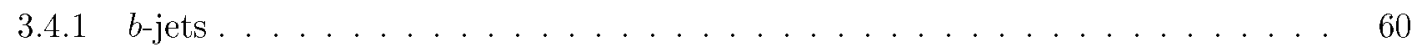

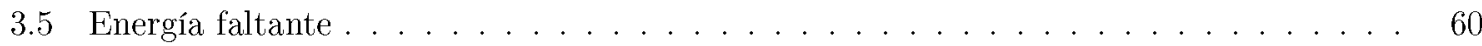


4 Métodos estadísticos para la búsqueda de nueva física $\quad 65$

4.1 Funciones de distribución de probabilidad . . . . . . . . . . . . . . . . 65

4.2 Estimadores . . . . . . . . . . . . . . . . . . . . . . . 66

4.3 Método del likelihood máximo . . . . . . . . . . . . . . . . . . . . 67

4.4 Contrastación de hipótesis . . . . . . . . . . . . . . . . . . . . . 67

4.5 Descubrimiento . . . . . . . . . . . . . . . . . . . . . . . . . . . . . . . 69

4.6 Límites de Exclusión . . . . . . . . . . . . . . . . . . . . . . . . 70

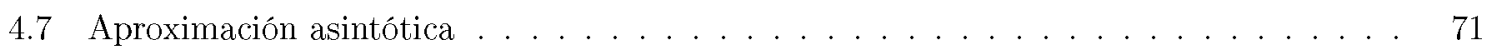

4.8 Significancia esperada . . . . . . . . . . . . . . . . . . . . . . . . . . . 72

5 Estrategia general del análisis $\quad 75$

5.1 Estrategia, señal y fondos del SM . . . . . . . . . . . . . . . . . . . . 75

5.2 Regiones de señal, control y validación . . . . . . . . . . . . . . . . . . . . . . 76

5.3 Extrapolación y factores de transferencia . . . . . . . . . . . . . . . . . 77

5.4 Construcción del modelo estadístico . . . . . . . . . . . . . . . . . . . . . . 78

5.5 Ajuste del modelo y resultados . . . . . . . . . . . . . . . . . . . . . 78

6 Modelo de señal y generación de eventos Monte Carlo $\quad 81$

6.1 Generación de eventos Monte Carlo . . . . . . . . . . . . . . . . . . . . . . . . . . . 81

6.1 .1 Espectro de masas y decaimientos . . . . . . . . . . . . . . . . 82

6.1 .2 Generador de eventos . . . . . . . . . . . . . . . . . . 82

6.1.3 Simulación del detector ATLAS . . . . . . . . . . . . . . . 84

6.2 Simulación de la señal de SUSY . . . . . . . . . . . . . . . . . . . . . . . . 84

6.2 .1 Estudios a nivel generador . . . . . . . . . . . . . . . 87

6.2 .2 Sección eficaz de producción . . . . . . . . . . . . . . . . . . 87

6.3 Simulación de los fondos del SM . . . . . . . . . . . . . . . . . . . 92

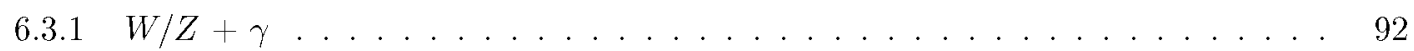

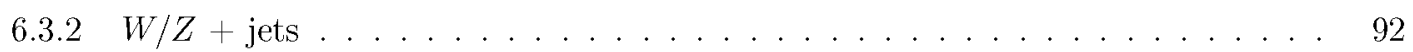

6.3 .3 Pares de tops $(+\gamma) \ldots \ldots \ldots \ldots \ldots 4 \ldots \ldots \ldots$

6.3 .4 Top $(+\gamma) \ldots \ldots \ldots \ldots \ldots . \ldots \ldots \ldots$

$6.3 .5 \gamma+$ jets y multijets . . . . . . . . . . . . . . . . . 95

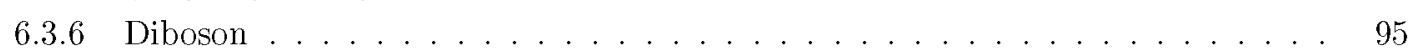

7 Selección de eventos: definición de las regiones de señal $\quad 97$

7.1 Criterios de calidad sobre los datos . . . . . . . . . . . . . . . . . . . . . 97

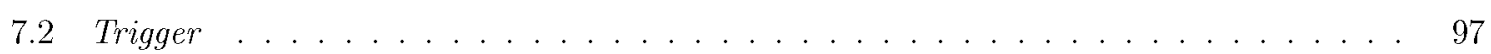

7.3 Preselección . . . . . . . . . . . . . . . . . . . . . . . . . . 98

7.3 .1 Vértice primario . . . . . . . . . . . . . . . . . . . 98

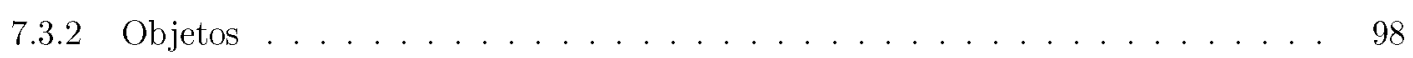

7.3.3 Eliminación de objetos superpuestos . . . . . . . . . . . . . . . . . . 99

7.4 Optimización de la selección de las regiones de señal . . . . . . . . . . . . . . . . 99

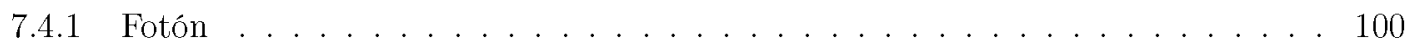

7.4 .2 Leptones . . . . . . . . . . . . . . . . . . . . . . . 101

7.4 .3 Energía faltante ......................... 101

7.4 .4 Multiplicidad y $p_{\mathrm{T}}$ de los jets . . . . . . . . . . . . . . . . . . . . . 102

7.4 .5 Separación angular entre jets y $E_{\mathrm{T}}^{\mathrm{miss}} \ldots \ldots \ldots \ldots$. . . . . . . . . 103 
7.4.6 Separación angular entre jets y fotón . . . . . . . . . . . . . . . . . 103

7.4 .7 Energía total transversa $\left(H_{\mathrm{T}}\right) \ldots \ldots \ldots \ldots \ldots \ldots \ldots$

7.4 .8 Variables de forma adicionales . . . . . . . . . . . . . . . 106

7.5 Selección final de las regiones de señal . . . . . . . . . . . . 106

7.6 Aceptancia y eficiencia . . . . . . . . . . . . . . . . . . . . 107

8 Estimación de los fondos $\quad 111$

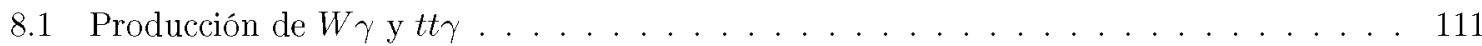

8.1 .1 Regiones de validación . . . . . . . . . . . . . . . . . . . . . . . . . 112

8.2 Producción de fotones directos $(\gamma+$ jets $) \ldots \ldots \ldots \ldots \ldots \ldots$

8.2 .1 Regiones de validación . . . . . . . . . . . . . . . . . . . . . . . . 114

8.3 Electrones identificados como fotones . . . . . . . . . . . . . . . . . . . 115

8.4 Jets identificados como fotones . . . . . . . . . . . . . . . . . . . . . . . 119

8.4.1 Descripción del método . . . . . . . . . . . . . . . . . . . . . . . . . 119

8.4 .2 Modelo de señal . . . . . . . . . . . . . . . . . . . . . . . 120

8.4 .3 Modelo de fondo . . . . . . . . . . . . . . . . . . . . . . . . . . 121

8.4.4 Ajuste combinado y estimación del fondo . . . . . . . . . . . . . . . . . 124

$9 \begin{array}{ll}9 \text { Resultados e interpretación } & 127\end{array}$

9.1 Análisis estadístico . . . . . . . . . . . . . . . . . . . . . 127

9.2 Incertezas sistemáticas . . . . . . . . . . . . . . . . . . . . . . 128

9.2 .1 Incertezas experimentales . . . . . . . . . . . . . . . . . . . . 128

9.2 .2 Incertezas teóricas . . . . . . . . . . . . . . . . . . . . . . . . . . 130

9.3 Ajuste simultáneo en las regiones de control . . . . . . . . . . . . . . . . . . 132

9.3.1 Resultados en las regiones de control . . . . . . . . . . . . . . . . . . . 133

9.3.2 Resultados en las regiones de validación . . . . . . . . . . . . . . . . . . . 134

9.3.3 Resultados en las regiones de señal . . . . . . . . . . . . . . . . . . . . . 135

9.3.4 Eventos en las regiones de señal . . . . . . . . . . . . . . . . . . . . 138

9.4 Límites a procesos de nueva física . . . . . . . . . . . . . . . . . . . . 145

9.5 Límites de exclusión en el modelo de SUSY considerado . . . . . . . . . . . . . . . 146

9.6 Búsqueda de señal de SUSY con datos a $\sqrt{s}=13 \mathrm{TeV} \ldots \ldots \ldots \ldots \ldots \ldots \ldots$

10 Conclusiones $\quad 151$

$\begin{array}{lr}\text { Agradecimientos } & 153\end{array}$

$\begin{array}{ll}\text { Bibliografía } & 155\end{array}$ 


\section{INTRODUCCIÓN}

Para explorar la región de energías del TeV, en el laboratorio CERN se construyó el Gran Colisionador de Hadrones (LHC) [1], diseñado para colisionar protones a una energía de centro de masa máxima de $\sqrt{s}=14 \mathrm{TeV}$ y una luminosidad que excede $\operatorname{los} 10^{34} \mathrm{~cm}^{-2} \mathrm{~s}^{-1}$. El LHC se puso en funcionamiento en el año 2010, marcando el inicio de una nueva era en el entendimiento de la naturaleza más fundamental y la búsqueda de nuevas partículas e interacciones. En el primer período de investigación del LHC, denominado Run 1, funcionó a $\sqrt{s}=7 \mathrm{TeV}$ durante el primer año, aumentando a $\sqrt{s}=8 \mathrm{TeV}$ a partir del año 2012 y hasta principios de 2013. Una vez realizadas las mejoras necesarias para alcanzar la energía y luminosidad nominales, en el 2015 se dio inicio al llamado Run 2, con una energía de centro de masa de $\sqrt{s}=13 \mathrm{TeV}$.

Uno de los detectores multipropósito del LHC es ATLAS ( A Torodial LHC AparatuS) [2], el detector de partículas de mayor volumen construido al presente (de $25 \mathrm{~m}$ de diámetro, $45 \mathrm{~m}$ de largo y con un peso de 7000 toneladas), que fue diseñado para estudiar un amplio espectro de fenómenos físicos en la escala de energía del TeV. Los haces de partículas del LHC colisionan en el centro de ATLAS generando miles de partículas desde el punto de interacción. Para la reconstrucción de las trazas de las partículas cargadas y los vértices de interacción ATLAS utiliza la información de un detector de píxeles, un detector de silicio y un detector de radiación de transición, que conjuntamente conforman el detector interno. El detector interno tiene una cobertura azimutal completa en la región de pseudo-rapidez $|\eta|<2.5$, y se encuentra inmerso en un campo magnético axial de $2 \mathrm{~T}$, el cual permite la reconstrucción del momento transverso de las partículas cargadas. La energía de los fotones y electrones es medida en el calorímetro electromagnético (EM), un detector de muestreo de argón líquido que cubre la región $|\eta|<3.2$. Para $|\eta|<2.5$ el calorímetro EM está dividido en tres capas longitudinales. La primera capa tiene una segmentación muy fina para facilitar la separación entre fotones y hadrones neutros, y para poder medir la dirección de las lluvias electromagnéticas, mientras que la mayor parte de la energía se deposita en la segunda capa. La energía de los jets es medida en los calorímetros EM y hadrónico. El calorímetro hadrónico está dividido en tres subregiones. La región central consiste en azulejos de centelleo activos y absorbentes de acero, mientras que las otras regiones se basan en tecnología de argón líquido. El calorímetro se encuentra rodeado por el espectrómetro de muones, el cual está inmerso en un campo magnético provisto por un sistema toroidal, y permite la reconstrucción y la determinación del momento de los muones en la región $|\eta|<2.7$.

Los eventos son retenidos para su posterior análisis utilizando un sistema de trigger de tres niveles [3] que identifica eventos consistentes con topologías de interés definidas previamente. Los algoritmos del nivel 1 están implementados en hardware, y utilizan solo una parte de la información del detector para reducir la tasa de eventos de $\mathcal{O}(1) \mathrm{GHz}$ a menos de $75 \mathrm{kHz}$. Los dos niveles siguientes, basados en software, utilizan toda la información del detector para refinar la selección de eventos, reduciendo finalmente la tasa de eventos a menos de $400 \mathrm{~Hz}$.

Uno de los grandes logros del siglo XX ha sido la revelación de teorías que describen todos los procesos de la naturaleza en términos de principios elegantes basados en consideraciones de simetría. El Modelo Estándar (SM, del inglés Standard Model) [4-6] de las partículas elementales y sus interacciones 
proporciona el marco indiscutible de la física de partículas actual. El acuerdo entre sus predicciones y los datos experimentales es excelente, en algunas casos con una precisión mayor al $1 \%$. Una de las predicciones críticas del SM está relacionada con el mecanismo de rompimiento espontáneo de la simetría electrodébil, necesaria para explicar el origen de las masas de los bosones de gauge $W$ y $Z$, mediadores de la fuerza débil, y de los fermiones [7,8]. Este mecanismo, denominado mecanismo de Brout-Englert-Higgs, introduce un campo escalar complejo que lleva a la predicción de una nueva partícula escalar, el bosón de Higgs. Uno de los resultados más importantes obtenidos con datos recolectados por ATLAS ha sido el histórico descubrimiento de una partícula consistente con el bosón predicho [9]. A pesar de los éxitos del SM, se cuenta con numerosos indicios empíricos y teóricos que promueven la existencia de nueva física a la escala del TeV y que conducen a interpretar al SM como el límite de bajas energías de alguna teoría que incluya nuevas interacciones. Uno de los problemas del SM se conoce como «problema de jerarquía» y está relacionado a la gran diferencia entre la escala electrodébil y la escala de Planck.

La observación del bosón de Higgs en los experimentos del LHC ha marcado el inicio de una nueva etapa, cambiando los objetivos de las investigaciones centradas en su búsqueda, a la fase de búsqueda de nuevas partículas e interacciones. La supersimetría (SUSY) [10-18] es una de las teorías con mayor motivación teórica para física más allá del Modelo Estándar. SUSY se presenta particularmente interesante ya que provee una solución natural a los dilemas teóricos del SM. En su realización mínima (el MSSM), se introduce una nueva partícula por cada bosón y fermión en el SM. Dado que estas partículas no han sido observadas experimentalmente, la nueva simetría debe estar rota en la naturaleza, presumiblemente a la escala del $\mathrm{TeV}$ (a fin de solucionar el llamado problema de fine-tuning). El MSSM posee 105 parámetros libres que se reducen suponiendo algún mecanismo de ruptura de la simetría. Los modelos de SUSY con rompimiento de supersimetría con mediación por campos de gauge (GMSB) [19-24] suponen un sector oculto en el cual se rompe la supersimetría y este rompimiento se comunica al sector visible a través de las interacciones usuales de bosones de gauge del SM. En estos modelos la partícula supersimétrica más liviana (LSP) es el gravitino $(\widetilde{G})$, el cual es muy liviano, y bajo ciertas condiciones resulta en un candidato viable de materia oscura. El espectro de masas de las partículas supersimétricas, y en particular la naturaleza de la segunda partícula más liviana (NLSP) que resulta ser el neutralino más liviano $\widetilde{\chi}_{1}^{0}$ para la mayor parte del espacio de parámetros, dictamina las partículas observables en el detector y los posibles canales de búsqueda. En años recientes, el esfuerzo para formular GMSB de una forma menos dependiente de los modelos llevó al desarrollo de lo que se conoce como General Gauge Mediation (GGM) [25] y su fenomenología comprende una gran variedad de estados finales, que motivaron el estudio realizado en esta tesis.

Esta tesis describe la búsqueda de SUSY en el marco de los modelos GGM con un fotón energético aislado, jets y gran cantidad de energía faltante en el estado final, con los datos recolectados en colisiones protón-protón $(p p)$ a una energía de centro de masa $\sqrt{s}=8 \mathrm{TeV}$ por el detector ATLAS del LHC durante el Run 1, correspondientes a una luminosidad total integrada de $20.3 \mathrm{fb}^{-1}$. Este estado final es complementario a otras búsquedas realizadas en ATLAS con estados finales de $\gamma \gamma+E_{\mathrm{T}}^{\text {miss }}[26,27]$, $\gamma+e / \mu+E_{\mathrm{T}}^{\text {miss }}[28], \gamma+b+E_{\mathrm{T}}^{\text {miss }}[29]$, y $Z+E_{\mathrm{T}}^{\text {miss }}$ [30]. También se han realizado búsquedas similares en CMS [31,32], aunque solo para el caso de neutralinos puramente bino o wino.

La tesis está estructurada en tres partes: (I) Motivación teórica, (II) Experimento y (III) Análisis de datos.

La primera parte contiene una descripción del marco teórico en el que se encuadra y que motiva el análisis realizado. El capítulo 1 incluye en la sección 1.1 los conceptos básicos del Modelo Estándar, haciendo énfasis en el buen acuerdo con los experimentos actuales de altas energías, finalizando con algunos de los problemas teóricos y experimentales que presenta y que motivan las teorías de nueva física. En la sección 1.2 se describen conceptos de Supersimetría, y en especial los modelos GMSB, en 
cuyo contexto se realizó el análisis presentado en este trabajo.

La segunda parte consiste en la descripción del experimento. En el capítulo 2 se describe el LHC y, en particular, el detector ATLAS, mientras que en el capítulo 3 se presentan los métodos utilizados para la reconstrucción e identificación de las partículas con el detector.

La tercera parte conforma la parte central de la tesis y describe el análisis específico realizado. En el capítulo 4 se explican los conceptos estadísticos fundamentales necesarios para la búsqueda de nueva física y los métodos desarrollados durante los últimos años para los experimentos del LHC. La estrategia general del análisis se describe en el capítulo 5 en donde se discute el modelo estadístico utilizado y la necesidad de definir regiones de señal, control y validación, en particular para la determinación del fondo contaminante de la señal de nueva física buscada. En el capítulo 6 se presenta el modelo de SUSY que motiva el estudio de esta tesis. También se presentan los detalles de las simulaciones Monte Carlo empleadas para la generación de los procesos del SM que conforman el fondo del análisis y las simulaciones de la señal de SUSY. La definición y optimización de las regiones de señal se describe en el capítulo 7 , mientras que en el capítulo 8 se presentan los métodos utilizados para la estimación del fondo en estas regiones. Las incertezas sistemáticas que afectan las medidas realizadas y los resultados obtenidos de esta investigación se discuten en el capítulo 9. Las conclusiones finales de esta tesis se presentan en el capítulo 10. 
PARTE I

MOTIVACIÓN TEÓRICA 



\section{MODELO ESTÁNDAR Y SUPERSIMETRÍA}

El Modelo Estándar es un marco teórico que describe las partículas elementales y sus interacciones a través de las fuerzas electromagnética, débil y fuerte. Es una teoría cuántica de campos, y sus predicciones, cuantitativas y cualitativas, han sido verificadas experimentalmente con gran precisión.

Una de las extensiones del SM mejor motivada desde el punto de vista teórico es la Supersimetría, ya que resuelve algunas de las limitaciones del mismo. En particular, provee una solución al problema de jerarquía, permite la unificación de las fuerzas del SM y hasta provee una conexión entre estas y la gravedad, además de proporcionar candidatos para la materia oscura.

En la sección 1.1 se describen brevemente las características más relevantes del SM y se detallan algunas de las dificultades que dan lugar a los modelos de física más allá del SM, mientras que en la sección 1.2 se introduce el concepto de Supersimetría y algunas de sus características fenomenológicas que resultan importantes para esta tesis.

\subsection{Conceptos básicos del Modelo Estándar}

\subsubsection{Las partículas elementales y sus interacciones}

De acuerdo al SM toda la materia está compuesta por un número pequeño de partículas fundamentales, fermiones, de espín $\frac{1}{2}$ que se dividen en dos tipos: «quarks ${ }^{*} »$ y «leptones» (ver Tabla 1.1). Hasta el momento, ningún experimento ha podido encontrar evidencia de que estos fermiones tengan una subestructura interna. Los quarks y leptones además están divididos en tres generaciones o familias, ordenadas según su masa. Las partículas de las generaciones más altas tienen mayor masa y son inestables, decayendo en partículas de generaciones más bajas. Es por este motivo que la materia ordinaria está formada por las partículas estables de la primera generación.

La distintas interacciones entre quarks y leptones son descriptas en el SM en términos del intercambio de partículas entre estos. Estas partículas de intercambio son los «bosones de gauge», y tienen espín igual a 1 (ver Tabla 1.2). Existen cuatro tipos de interacciones fundamentales. La interacción fuerte es la responsable de mantener los quarks formando los protones, neutrones y hadrones en general, y es mediada por partículas no masivas llamadas gluones. Las interacciones electromagnéticas son las responsables de los fenómenos extra nucleares, como por ejemplo las fuerzas intermoleculares en líquidos y sólidos. Estas interacciones están mediadas por el intercambio de fotones no masivos. La interacción débil es la responsable de los procesos de desintegración de núcleos y partículas, y sus mediadores son los bosones $Z^{0}$ y $W^{ \pm}$, con masas del orden de 100 veces la masa del protón. Por último existe una interacción que actúa entre todo tipo de partículas, la interacción gravitatoria. Actualmente no

\footnotetext{
"Los extranjerismos "quarks", "jets" y los nombres de las partículas supersimétricas se escribirán a continuación sin cursiva.
} 
Tabla 1.1: Partículas elementales de materia del SM, incluyendo las tres generaciones, ordenadas según su masa. En la segunda y tercer columna se encuentra la masa [33] y la carga eléctrica, respectivamente. En el caso de los neutrinos solo existen cotas superiores de su masa.

\begin{tabular}{clllrrrr}
\hline & \multicolumn{3}{c}{ Partícula } & & Masa & $r$ & $\begin{array}{r}\text { Carga } \\
\text { Eléctrica }\end{array}$ \\
\hline \multirow{2}{*}{ Leptones } & $\mathrm{e}$ & $\mu$ & $\tau$ & $511 \mathrm{keV}$ & $105.7 \mathrm{MeV}$ & $1768 \mathrm{MeV}$ & -1 \\
& $\nu_{e}$ & $\nu_{\mu}$ & $\nu_{\tau}$ & $<2.2 \mathrm{eV}$ & $<0.17 \mathrm{MeV}$ & $<15.5 \mathrm{MeV}$ & 0 \\
\hline \multirow{2}{*}{ Quarks } & $u$ & $c$ & $t$ & $2.4 \mathrm{MeV}$ & $1.27 \mathrm{GeV}$ & $171.2 \mathrm{GeV}$ & $\frac{2}{3}$ \\
& $d$ & $s$ & $b$ & $4.8 \mathrm{MeV}$ & $104 \mathrm{MeV}$ & $4.2 \mathrm{GeV}$ & $-\frac{1}{3}$ \\
\hline
\end{tabular}

existe ninguna teoría cuántica completa que explique esta interacción fundamental, aunque hay varias teorías propuestas que postulan la existencia de una partícula de espín 2 mediadora de la gravedad, denominada gravitón. En la escala de los experimentos de partículas, la gravitatoria es la interacción más débil de todas las interacciones fundamentales, aunque es la dominante en la escala del universo, y será despreciada en lo que sigue.

Los leptones interactúan de forma débil y electromagnética en el caso de ser cargados, o solo débilmente si son neutros. En contraste, los quarks interactúan además de débil y electromagnéticamente, por medio de la interacción fuerte. Esta es la distinción fundamental entre quarks y leptones.

Adicionalmente, por cada partícula en el SM, existe una antipartícula asociada, con todos sus números cuánticos no nulos opuestos. Los bosones de gauge neutros constituyen su propia antipartícula.

Tabla 1.2: Bosones de gauge mediadores de las diferentes interacciones fundamentales incluidas en el $\mathrm{SM}$, junto con su masa [33] y carga eléctrica.

\begin{tabular}{ccrr}
\hline Fuerza & Partícula & Masa & Carga Eléctrica \\
\hline \multirow{2}{*}{ Débil } & $W^{ \pm}$ & $80.385 \pm 0.015 \mathrm{GeV}$ & \pm 1 \\
& $Z^{0}$ & $91.1876 \pm 0.0021 \mathrm{GeV}$ & 0 \\
\hline Electromagnética & $\gamma$ & 0 & 0 \\
\hline Fuerte & $g$ & 0 & 0 \\
\hline
\end{tabular}

Formalmente, el Modelo Estándar es una teoría cuántica de campos renormalizable que provee una descripción de los campos de las partículas elementales, y las interacciones fuerte, débil y electromagnética. Estas interacciones surgen del requerimiento de que la teoría sea invariante bajo transformaciones de gauge locales del grupo de simetría:

$$
\mathrm{SU}(3)_{\mathrm{C}} \times \mathrm{SU}(2)_{\mathrm{L}} \times \mathrm{U}(1)_{\mathrm{Y}}
$$

El subgrupo $\mathrm{SU}(2)_{\mathrm{L}} \times \mathrm{U}(1)_{\mathrm{Y}}$ representa el sector electrodébil, es decir, la electrodinámica cuántica (QED) más las interacciones débiles, donde $\mathrm{L}^{*}$ e Y se refieren al isoespín débil y la hipercarga débil, que son las cargas de $\mathrm{SU}(2)$ y U(1), respectivamente. La adición del grupo $\mathrm{SU}(3)_{\mathrm{C}}$ incluye la cromodinámica cuántica (QCD), que es la teoría de campos de gauge que describe las interacciones fuertes de los quarks y gluones que poseen carga de color, indicada por $\mathrm{C}$.

La masa de las partículas en el SM es introducida mediante el llamado mecanismo de Brout-EnglertHiggs [7,8], vía la ruptura espontánea de la simetría electrodébil:

\footnotetext{
${ }^{*} \mathrm{~L}$ indica que $\mathrm{SU}(2)_{\mathrm{L}}$ actúa solo sobre los fermiones de quiralidad izquierda
} 


$$
\mathrm{SU}(3)_{\mathrm{C}} \times \mathrm{SU}(2)_{\mathrm{L}} \times \mathrm{U}(1)_{\mathrm{Y}} \rightarrow \mathrm{SU}(3)_{\mathrm{C}} \times \mathrm{U}(1)_{\mathrm{EM}}
$$

que resulta en la generación de los bosones de gauge masivos $W^{ \pm}$y $Z$. En este mecanismo, además, un nuevo campo escalar debe ser agregado al lagrangiano, dando lugar a la aparición de un nuevo bosón masivo de espín 0, al que se lo llamó «bosón de Higgs» $(H)$.

Weinberg y Salam fueron los primeros en aplicar este mecanismo al rompimiento de la simetría electrodébil $[4,5]$ y mostraron cómo este mecanismo podía ser incorporado a la teoría electrodébil de Glashow [6], dando inicio a lo que hoy se conoce como el Modelo Estándar de la física de partículas.

La relación entre las masas de los bosones $W^{ \pm}$y $Z$ predicha por el SM está dada por $m_{W} / m_{Z}=$ $\cos \theta_{W}$, donde $\theta_{W}$ es el ángulo de mezcla de Weinberg, y relaciona la constante de acoplamiento débil (g) con la electromagnética $\left(g^{\prime}\right) \operatorname{comotan} \theta_{W}=g^{\prime} / g$. Los bosones $W^{ \pm}$y $Z$ fueron descubiertos en 1982 por las colaboraciones UA1 y UA2 del experimento SppS del CERN.

No solo los bosones de gauge adquieren masa debido al mecanismo de Brout-Englert-Higgs, también lo hacen los fermiones que forman la materia. El descubrimiento del quark top en 1995 por las colaboraciones D0 y CDF, con una masa de $\sim 173 \mathrm{GeV}$, completó las familias de las partículas que conforman la materia.

Desde el punto de vista teórico la masa del bosón de Higgs es un parámetro libre dentro del SM y por lo tanto ninguna predicción puede ser hecha sobre su valor. La búsqueda del bosón de Higgs, la única partícula del SM que no había sido descubierta aún (hasta 2012), fue uno de los grandes objetivos por los cuales se diseñó y construyó el LHC. En el año 2012 el CERN anunció el descubrimiento de una partícula consistente con el bosón de Higgs por parte de los dos grandes experimentos del LHC, ATLAS y CMS [9,34]. La medición combinada entre ATLAS y CMS de la masa del Higgs es $125.09 \pm 0.21$ (stat.) \pm 0.11 (sist.) GeV [35]. El detalle de estas mediciones puede verse en la Figura 1.1. El descubrimiento del bosón de Higgs completó el espectro de partículas del SM.

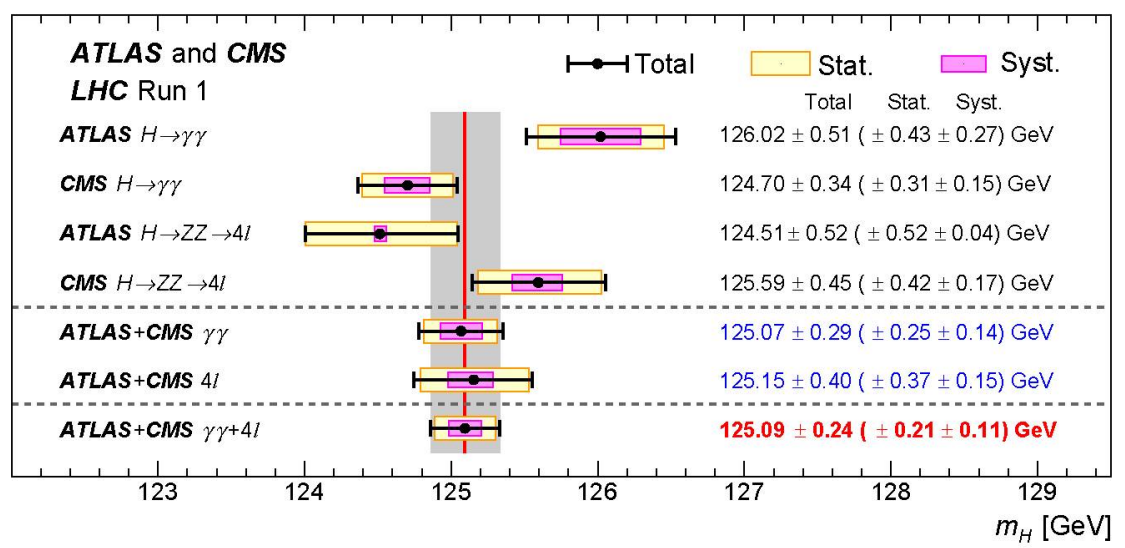

Figura 1.1: Resumen de las mediciones de la masa del bosón de Higgs de los distintos análisis de ATLAS y CMS, y del análisis combinado. Se indican las incertezas sistemáticas (bandas de color magenta), estadísticas (bandas de color amarillo), y total (líneas negras). La línea roja vertical y la correspondiente sombra gris indican el valor central y la incerteza total de la medida combinada, respectivamente [35]. 


\subsubsection{QCD y colisiones $p p$ en el LHC}

La cromodinámica cuántica (QCD) [36] es la teoría de campos de gauge renormalizable que describe la interacción fuerte entre las partículas que poseen carga de color: quarks y gluones. A diferencia de QED, los mediadores de esta interacción, los gluones, poseen carga de color, por lo que pueden interactuar entre ellos, dando lugar a un comportamiento inusual de la interacción fuerte. La fuerza fuerte aumenta linealmente con la distancia entre dos cargas. La constante de acoplamiento $\alpha_{s}$ depende entonces de la distancia entre las cargas o de la escala de energía de la interacción. Se dice que la constante "corre", siendo grande a bajas energía (o grandes distancias) y haciéndose más chica a altas energías (o menores distancias).

El efecto neto de esta característica del acoplamiento fuerte es el confinamiento, es decir, que las partículas con color no puedan existir libremente. Solo estados de color neutro de múltiples partículas de color pueden ser observados en la naturaleza viajando distancias macroscópicas. Los estados que consisten en un par quark-antiquark son denominados mesones y los formados por tres quarks son denominados bariones. El protón en un barión constituido por dos quarks u y un quark $d$, donde cada quark tiene uno de las tres posibles cargas de color para dejar un estado neutro. Estos tres quarks son llamados quarks de valencia del protón, y están rodeados por un mar de gluones y pares de quark-antiquark que surgen de fluctuaciones cuánticas. Otra consecuencia de la estructura de la interacción fuerte es que los cálculos perturbativos no son posibles en el régimen de grandes valores de $\alpha_{s}$.

El LHC es un colisionador de protones, por lo tanto es esencial una precisa descripción de la estructura del protón, ya que una colisión $p p$ a altas energías es básicamente una colisión de dos constituyentes del mismo. A altas energías es posible entonces aplicar el llamado «modelo de partones», en el cual los hadrones están compuestos por partículas puntuales. Este modelo fue introducido por Feynman [37] y Bjorken [38] a fines de los años 60, para interpretar los resultados de los experimentos de dispersión inelástica profunda (DIS) electrón-nucleón en SLAC. Esta descripción ha probado ser una buena aproximación para las interacciones partón-partón de gran trasferencia de momento pero no es apropiado para modelar la interacción a bajas energías. Los quarks de valencia y los quarks y antiquarks del mar junto con los gluones son llamados «partones» del protón. Cada partón lleva solo una fracción del momento y la energía del protón. Para la medición de una sección eficaz de dispersión fuerte que involucre quarks y gluones en el estado inicial, es necesario conocer el momento de las partículas incidentes. Como los partones solo llevan una fracción del momento del protón, y están en interacción permanente entre ellos, el momento es desconocido, por lo que la escala de energía $Q$ de las colisiones varía. Además, como se mencionó, los quarks $(q)$ y gluones $(g)$ salientes no pueden observarse directamente debido al confinamiento, pero son observados en el detector como jets. Entonces no es posible medir una sección eficaz partónica como $\sigma(q g \rightarrow q g)$, pero se puede hacer una medida inclusiva, como la sección eficaz hadrónica $\sigma(p p \rightarrow j j)$ con dos jets en el estado final. En teoría de perturbaciones, para pasar desde la sección eficaz partónica a la sección eficaz hadrónica es necesario conocer la probabilidad de que un partón de tipo $n$ sea encontrado con una fracción de momento $x$, es decir, las funciones de distribución partónica (PDF). Estas funciones son determinadas a partir de datos obtenidos de los propios experimentos de altas energías, ya que no pueden determinarse a partir de la teoría.

Esta conexión entre los hadrones observables y el nivel partónico es posible gracias al concepto de «factorización», que permite una separación sistemática entre las interacciones de corta distancia (de los partones) y las interacciones de larga distancia (responsables del confinamiento de color y la formación de hadrones). El teorema de factorización [39] establece que la sección eficaz de producción de cualquier proceso de QCD del tipo $A+B \rightarrow X$, siendo $a_{i}\left(b_{j}\right)$ los constituyentes del hadrón inicial 
$A(B)$, puede ser expresada como:

$$
\sigma_{A B \rightarrow X}=\sum_{i j} \int d x_{a_{i}} d x_{b_{j}} f_{A / a_{i}}\left(x_{a_{i}}, \mu_{F}^{2}\right) f_{B / b_{j}}\left(x_{b_{j}}, \mu_{F}^{2}\right) \sigma_{a_{i} b_{j} \rightarrow X}\left(\mu_{F}^{2}, \mu_{R}^{2}\right)
$$

donde $x_{i}\left(x_{j}\right)$ es la fracción del momento del hadrón $A(B)$ que lleva el partón $a_{i}\left(b_{j}\right)$ y $\sigma_{a_{i} b_{j} \rightarrow X}$ es la sección eficaz de la interacción a nivel partónico, calculada a un dado orden de perturbaciones y una escala de renormalización $\mu_{R}$. La escala de renormalización es introducida para absorber las divergencias ultravioletas que aparecen en los cálculos perturbativos más allá de LO. Las funciones $f_{h / n}\left(x_{n}, \mu_{F}^{2}\right)$ son las PDF, que representan la probabilidad de encontrar un partón de tipo $n$ en el hadrón $h$ con una fracción de momento $x_{n}$, dada una escala de factorización $\mu_{F}$. Esta escala es un parámetro arbitrario introducido para tratar singularidades que aparecen en el régimen no perturbativo. Estas divergencias son absorbidas, en forma similar a la renormalización, dentro de las funciones de distribución partónicas a la escala $\mu_{F}$. Si bien las PDF no pueden ser determinadas perturbativamente, se puede predecir su dependencia con el impulso transferido $Q^{2}$ por medio de las ecuaciones de evolución DGLAP [40-42]. De esta forma, la medida experimental de su forma funcional a un dado $Q_{0}^{2}$ fijo permite obtener predicciones de las PDF para un amplio espectro de $Q^{2}$.

A modo de ejemplo, en la Figura 1.2, se muestra el buen acuerdo entre la sección eficaz de algunos procesos del SM medidas por ATLAS y las predicciones teóricas. Las observaciones experimentales realizadas en LHC resultan compatibles con el SM a un nivel de muy alta precisión.

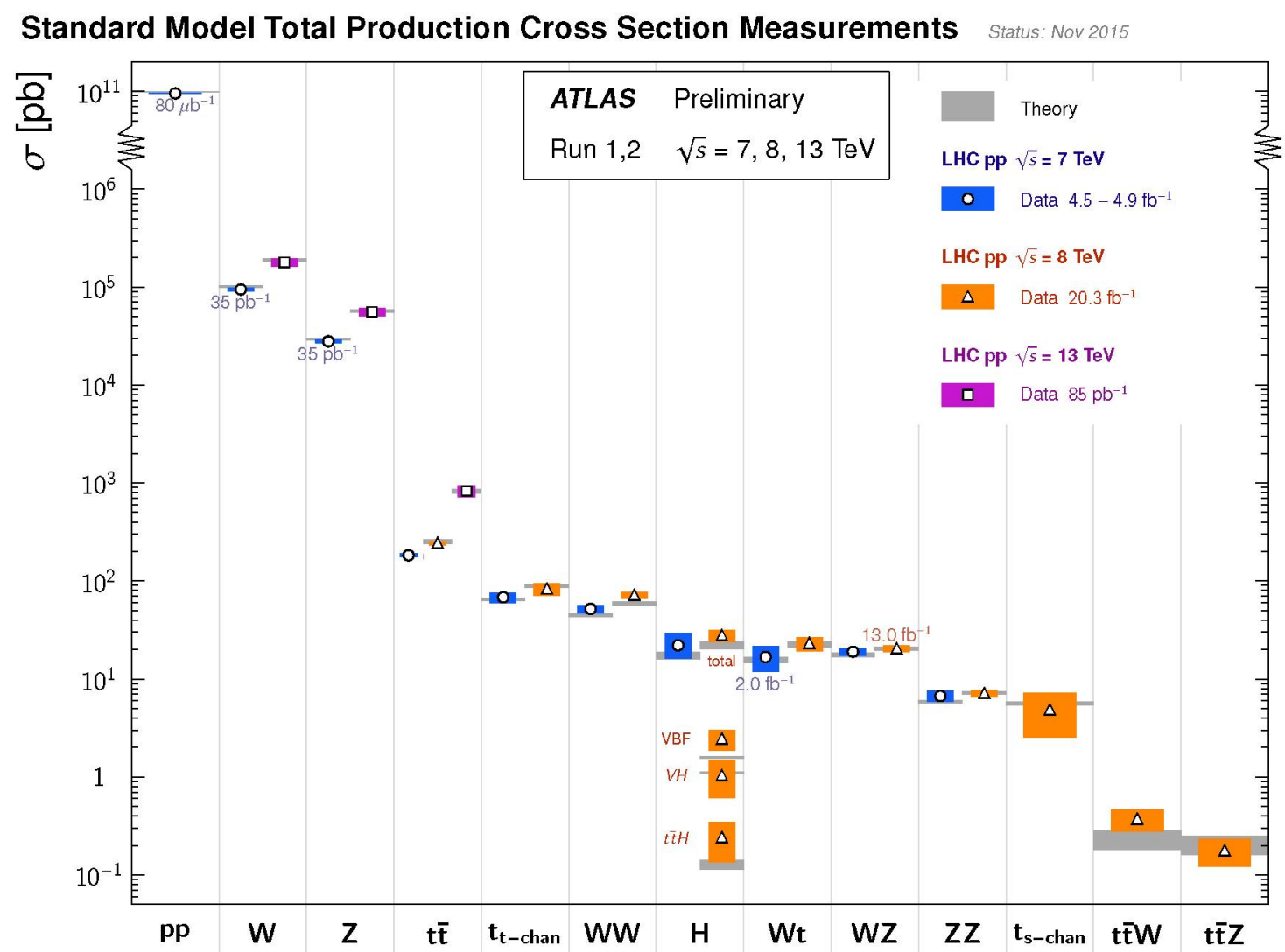

Figura 1.2: Resumen de las distintas medidas de sección eficaz de producción de procesos del SM, comparadas con sus valores teóricos esperados. Los valores teóricos esperados fueron calculados como mínimo a NLO [43]. 


\subsubsection{Física más allá del SM}

El SM provee una descripción notablemente exitosa de todos los fenómenos accesibles con los experimentos de altas energías disponibles actualmente. Sin embargo, también se sabe que el SM deja cuestiones sin resolver, tanto desde el punto de vista teórico, como experimental.

El hecho de que existan cuatro tipos de interacciones distintas e independientes es un poco insatisfactorio y desde Einstein se ha especulado que ellas sean distintas manifestaciones de un único campo unificado. El descubrimiento de los bosones $Z$ y $W$, tal como fueron predichos por la teoría electrodébil, significaron un triunfo de la idea de una teoría unificada de las fuerzas. Cabe destacarse que los resultados de las medidas de precisión de la sección eficaz diferencial de las corrientes cargadas y neutras en la dispersión inelástica profunda, obtenidos en HERA [44], proporcionaron una clara evidencia de la unificación electrodébil.

Todo parece indicar que el SM es una teoría efectiva a bajas energías, muy precisa hasta escalas de energía del orden de los $100 \mathrm{GeV}$. Sin embargo, los físicos consideran que el éxito del SM no se extenderá a energías mayores. Esta idea impulsa intentos de incorporar el SM en una teoría más fundamental. Incluso ante la ausencia de la gran unificación de las fuerzas electrodébil y fuerte a una escala muy alta de energía, el SM debería ser modificado para incorporar los efectos de la gravedad a la escala de Planck.

Otro síntoma de incompletitud es la gran cantidad de parámetros libres que deben ajustarse a los datos observados, ya que no resultan de principios teóricos más fundamentales. El SM tiene 19 parámetros libres: 13 del sector de Yukawa, 2 del sector de Higgs, las tres constantes de acoplamiento, y una fase del lagrangiano de QCD.

En este contexto también resulta inexplicable por qué el cociente entre la escala electrodébil y la escala de Planck $M_{W} / M_{P} \sim 10^{-17}$ es tan chico, lo que se conoce como «problema de jerarquía». Por otro lado, en el SM, la escala de las interacciones electrodébiles se derivan de un campo escalar elemental que adquiere un valor de expectación de vacío $v=2 M_{W} / g=246 \mathrm{GeV}$. Sin embargo, si se acopla una teoría de partículas escalares a nueva física a alguna escala arbitraria $\Lambda$, las correcciones radiativas al cuadrado de la masa escalar son del orden de $\Lambda^{2}$, debido a las divergencias cuadráticas en la autoenergía, lo cual indica la sensibilidad cuadrática a la mayor escala de energía de la teoría. Por esto, la masa "natural" de cualquier partícula escalar es $\Lambda$. Así, para tener una teoría electrodébil exitosa, la masa del Higgs debe ser del orden de la escala electrodébil. Este hecho, que la masa del bosón de Higgs no puede ser igual a su valor natural de $\Lambda=M_{P}$, es llamado «problema de naturalidad».

Desde el punto de vista experimental, también existen algunos resultados que no pueden acomodarse dentro del SM. El SM considera a los neutrinos como partículas no masivas, pero distintos experimentos $[45,46]$ han observado que los mismos presentan oscilaciones de sabor, lo que puede explicarse en el caso en que estos tengan una masa no nula. Por el momento solo existen límites superiores para estas masas (ver Tabla 1.1), que son muy pequeñas comparadas con las de los demás fermiones. El término de masa para fermiones puede escribirse usando el doblete de Higgs si existen grados de libertad asociados con los fermiones de quiralidad izquierda y derecha para un dado sabor. La masa obtenida por esta interacción es llamada masa de Dirac. Para que este mecanismo puede ser utilizado en los neutrinos, deberían existir los neutrinos de quiralidad derecha.

El SM tampoco provee un candidato para explicar la naturaleza de la materia oscura. La existencia de la materia oscura fue inferida por primera vez como resultado de las inconsistencias observadas entre la masa estimada de las curvas de rotación galácticas y de su luminosidad [47]. Solo el $4 \%$ del universo consiste de la materia que conocemos [48], cerca del $23 \%$ es materia oscura, y el restante $73 \%$ es energía oscura. La única partícula del SM que podría ser un candidato viable de materia oscura es el neutrino, pero como su masa es muy chica para poder explicar estos fenómenos, ha sido descartado. 
Son varias las teorías que intentan explicar parcial o totalmente los problemas mencionados anteriormente. Estas se conocen como teorías de física más allá del SM y entre ellas se encuentra la Supersimetría, modelos con dimensiones extra, teoría de cuerdas, teorías tecnicolor, etc. En la siguiente sección se explica brevemente en qué consiste una de las teorías de física más allá del SM más motivadas desde el punto de vista teórico, que es la Supersimetría.

\subsection{Supersimetría}

El Modelo Estándar de la física de altas energías, descripto en la sección 1.1, ha tenido un gran éxito en la descripción de los fenómenos conocidos hasta la escala del TeV explorados por los experimentos en los últimos años. A pesar de esto, no hay dudas respecto a que es necesaria una nueva teoría a la escala reducida de Planck $M_{P}$, donde los efectos cuánticos gravitacionales son importantes. El solo hecho de que la relación $M_{W} / M_{P}$ es tan chica es una gran motivación para la física más allá del Modelo Estándar («problema de jerarquía») [49]. Esta no es una dificultad intrínseca del SM, sino una preocupante sensibilidad del potencial de Higgs a nueva física en casi cualquier extensión imaginable del SM.

La parte eléctricamente neutra del campo de Higgs del SM es un escalar complejo $H$ con un potencial clásico $V=m_{H}^{2}|H|^{2}+\lambda|H|^{4}$. El SM necesita un valor de expectación del vacío (VEV) para $H$ no nulo, en el mínimo del potencial. Esto ocurre si $\lambda>0$ y $m_{H}^{2}<0$, resultando en $\langle H\rangle=\sqrt{-m_{H}^{2} / 2 \lambda}$. Experimentalmente, de las medidas de las propiedades de las interacciones débiles, se sabe que el valor de $\langle H\rangle$ es de aproximadamente $174 \mathrm{GeV}$. El descubrimiento del bosón de Higgs en el 2012 con una masa cercana a $125 \mathrm{GeV}$ implica que, suponiendo que el SM es correcto como una teoría efectiva, $\lambda=0.126$ y $m_{H}^{2}=-(92.9 \mathrm{GeV})^{2}$.

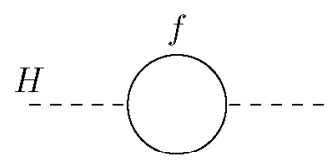

Figura 1.3: Correcciones cuánticas a un loop al parámetro de masa del Higgs $m_{H}^{2}$ debido a la masa de un fermión de Dirac $f$.

El problema es que $m_{H}^{2}$ recibe grandes correcciones cuánticas de los efectos virtuales de cada partícula a la que se acopla, directa o indirectamente, el campo de Higgs. Por ejemplo, en la Figura 1.3 se tiene una corrección a $m_{H}^{2}$ del loop que contiene un fermión de Dirac $f$ con masa $m_{f}$. Si el campo de Higgs se acopla a $f$ con un término en el lagrangiano igual a $-\lambda_{f} H \bar{f} f$, el diagrama de Feynman en la Figura 1.3 genera una corrección:

$$
\Delta m_{H}^{2}=-\frac{\left|\lambda_{f}\right|^{2}}{8 \pi^{2}} \Lambda_{\mathrm{UV}}^{2}+\ldots
$$

donde $\Lambda_{\mathrm{UV}}$ es el corte usado para regular la integral en el loop. Debe ser interpretado como la mínima escala de energía a la cual entra la nueva física para alterar el comportamiento de la teoría a altas energías. Los puntos suspensivos representan términos proporcionales a $m_{f}^{2}$, que crecen a lo sumo logarítmicamente con $\Lambda_{\mathrm{UV}}$. Cualquiera de los leptones o quarks del SM puede jugar el rol de $f$ (para el caso de quarks la corrección tiene que ser multiplicada por 3 para tener en cuenta el color) y la corrección más grande será cuando $f$ es el quark top con $\lambda_{f} \approx 1$.

El problema aparece si $\Lambda_{\mathrm{UV}}$ es del orden de $M_{P}$, ya que la corrección a $m_{H}^{2}$ es 30 órdenes de 
magnitud más grande que el valor requerido de $m_{H}^{2} \sim(100 \mathrm{GeV})^{2}$. Este es solo un problema para las correcciones al cuadrado de la masa del bosón de Higgs escalar, porque las correcciones cuánticas a las masas de los fermiones y los bosones de gauge no tienen una sensibilidad cuadrática directa a $\Lambda_{\mathrm{UV}}$ como la que está en la ecuación (1.4). Sin embargo, los quarks, leptones y los bosones de gauge electrodébiles $Z^{0}, W^{ \pm}$del SM, todos obtienen masa de $\langle H\rangle$, por lo tanto el espectro completo de masas del SM es directa o indirectamente sensible a la escala de corte $\Lambda_{\mathrm{UV}}$.

Se puede pensar que la solución es elegir un $\Lambda$ no demasiado grande, pero igualmente se debería mezclar todavía algo de nueva física a la escala $\Lambda_{\mathrm{UV}}$ que no solo altere los propagadores en el loop, sino que corte la integral. Esto no es fácil en una teoría cuyo lagrangiano no contiene más de dos derivadas, y las teorías de mayor orden en derivadas generalmente sufren de fallas de unitariedad o causalidad.

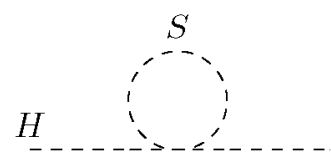

Figura 1.4: Correcciones cuánticas a un loop al parámetro de masa del Higgos $m_{H}^{2}$ debido a la masa de un campo escalar $S$.

En el caso de que exista un escalar complejo pesado $S$ con masa $m_{S}$ que se acopla al Higgs con un término en el lagrangiano $-\lambda_{S}|H|^{2}|S|^{2}$, el diagrama de Feynman es el que se muestra en la Figura 1.4 y este da lugar a una corrección:

$$
\Delta m_{H}^{2}=\frac{\lambda_{S}^{2}}{16 \pi^{2}}\left[\Lambda_{\mathrm{UV}}^{2}-2 m_{S}^{2} \ln \left(\Lambda_{\mathrm{UV}}^{2} / m_{S}\right)+\ldots\right]
$$

Si el bosón de Higgs es una partícula fundamental y hay nueva física a una escala mucho mayor que la escala electrodébil es necesario algún tipo de cancelación entre las distintas contribuciones a $\Delta m_{H}^{2}$.

La cancelación sistemática de las contribuciones a $\Delta m_{H}^{2}$ puede ser realizada por una simetría. Comparando las ecuaciones (1.4) y (1.5) se puede ver que existe una diferencia de signo entre las contribuciones del loop fermiónico y bosónico. Si se considera la existencia de una simetría que relacione fermiones y bosones, la cancelación de todas estas contribuciones a las masas escalares no solo es posible, sino que es inevitable. A esta simetría se la denomina supersimetría o SUSY [49].

Una transformación supersimétrica convierte un estado bosónico en uno fermiónico, y viceversa. El operador $Q$ que genera estas transformaciones debe ser un espinor anticonmutativo, con

$$
Q \mid \text { bosón }\rangle=\mid \text { fermión }\rangle, \quad Q \mid \text { fermión }\rangle=\mid \text { bosón }\rangle
$$

Los espinores son intrínsecamente objetos complejos, por lo tanto el conjugado hermítico de $Q$ es también un generador de la simetría. Debido a que $Q$ y $Q^{\dagger}$ son operadores fermiónicos, llevan momento angular de espín $\frac{1}{2}$, por lo tanto es claro que SUSY debe ser una simetría espacio-temporal y los operadores $Q$ y $Q^{\dagger}$ deben satisfacer un álgebra de la siguiente forma,

$$
\begin{aligned}
\left\{Q, Q^{\dagger}\right\} & =P^{\mu} \\
\{Q, Q\} & =\left\{Q^{\dagger}, Q^{\dagger}\right\}=0 \\
{\left[P^{\mu}, Q\right] } & =\left[P^{\mu}, Q^{\dagger}\right]=0
\end{aligned}
$$

donde $P^{\mu}$ es el momento generador de las traslaciones espacio-temporales. 
Los estados de partícula de una teoría supersimétrica son representados en el álgebra de SUSY como supermultipletes. Cada supermultiplete contiene ambos estados, fermión y bosón, que son comúnmente llamados supercompañeros uno de otro.

Los generadores $Q$ y $Q^{\dagger}$ conmutan con los generadores de las transformaciones de gauge, por lo tanto las partículas en un mismo supermultiplete tienen que estar en la misma representación del grupo de gauge, y tener la misma carga eléctrica, isoespín y color. Y como el operador de masa $-P^{2}$ también conmuta con los generadores y con todos los operadores de rotación y traslación, deberán tener los mismos autovalores de $-P^{2}$, y entonces la misma masa.

Es fácil probar que cada supermultiplete tiene que contener igual número de grados de libertad fermiónico que bosónico, $n_{B}=n_{F}$. La posibilidad más simple para satisfacer esto es tener un único fermión de Weyl $\left(n_{F}=2\right)$ y dos escalares reales (cada uno con $n_{B}=1$ ). Estos dos escalares se suelen poner como un único campo escalar complejo. Esta combinación de un fermión de Weyl de dos componentes y un campo escalar complejo es llamado un supermultiplete quiral (ó escalar).

Otra posibilidad es que el supermultiplete contenga un bosón vectorial de espín 1. Para que la teoría sea renormalizable, tiene que ser un bosón de gauge no masivo, al menos antes de que la simetría de gauge sea espontáneamente rota. En este caso, este bosón contiene dos estados de helicidad, $n_{B}=2$. Por lo tanto su supercompañero es un fermión de Weyl de espín $\frac{1}{2}$, con dos estados de helicidad, $n_{F}=2$. Si en vez de esto, se intenta usar un fermión de espín $\frac{3}{2}$ la teoría no sería renormalizable. Los bosones de gauge deben transformar como la representación adjunta del grupo de gauge, por lo que sus compañeros fermiónicos, llamados «gauginos», también. Esta combinación de gauginos de espín $\frac{1}{2}$ y bosones de gauge de espín 1 es llamada supermultiplete de gauge (ó vectorial). En el caso de incluir la gravedad, el gravitón de espín 2 (con dos estados de helicidad, $n_{B}=2$ ) tiene un supercompañero de espín $\frac{3}{2}$ llamado «gravitino».

Existen otras combinaciones posibles de partículas que pueden satisfacer estas condiciones, pero siempre se reducen a combinaciones de supermultipletes quirales o de gauge, excepto en ciertas teorías con supersimetría extendida. Estas teorías tienen más de una copia de los generadores $Q$ y $Q^{\dagger}$, pero no son muy prometedoras desde el punto de vista fenomenológico. La teoría no extendida y fenomenológicamente viable es llamada generalmente $N=1$, donde $N$ se refiere al número de supersimetrías (el número de las distintas copias de $Q$ y $Q^{\dagger}$ ).

\subsubsection{Modelo Estándar Supersimétrico Mínimo}

En una extensión supersimétrica del SM, cada una de las partículas elementales conocidas está contenida en un supermultiplete quiral o de gauge, que debe compartir con un supercompañero con espín que difiera en $\frac{1}{2}$. La extensión que requiere la introducción de la mínima cantidad de partículas se conoce como «Modelo Estándar Supersimétrico Mínimo», o MSSM.

Para entender las consecuencias fenomenológicas del MSSM, es necesario conocer cómo las distintas partículas encajan en los supermultipletes y atribuirles nombres apropiados. Solo los supermultipletes quirales pueden contener fermiones cuya parte izquierda y derecha transforman de forma diferente bajo el grupo de gauge. Todos los fermiones del SM (quarks y leptones) tienen esta propiedad, por lo tanto deben ser miembros de supermultipletes quirales. Los nombres de los compañeros de espín 0 de los quarks o leptones son construidos anteponiendo una "s" (de scalar), y son llamados «squarks»y «sleptones». La parte izquierda y derecha de los quarks y leptones son fermiones de Weyl con diferentes propiedades de transformación de gauge del SM, entonces cada uno debe tener un compañero escalar complejo. Por ejemplo, los supercompañeros de la parte izquierda y derecha del campo de Dirac de los electrones son llamadas $\widetilde{e}_{\mathrm{L}}$ y $\widetilde{e}_{\mathrm{R}}$, aunque el subíndice no se refiere a la helicidad de los slectrones (ya que tiene espín 0) sino a la de sus supercompañeros. Lo mismo ocurre para $\widetilde{\mu}_{\mathrm{L}}, \widetilde{\mu}_{\mathrm{R}}, \widetilde{\tau}_{\mathrm{L}} \mathrm{y} \widetilde{\tau}_{\mathrm{R}}$. Los 
neutrinos del SM son siempre izquierdos por lo que sus supercompañeros se denotan $\widetilde{\nu}$. Para el caso de los quarks se tiene a $\widetilde{q}_{\mathrm{L}}$ y $\widetilde{q}_{\mathrm{R}}$, con $q=u, d, s, c, b, t$. Las interacciones de gauge de cada uno de los campos de squarks y sleptones son las mismas que la de los correspondientes fermiones del SM.

El bosón escalar de Higgs debe estar en un supermultiplete quiral ya que tiene espín 0. Dada la naturaleza de los campos quirales introducidos en la implementación de SUSY, el campo escalar de Higgs no es suficiente para dar masa a los fermiones de helicidad izquierda y derecha. Se debe agregar un nuevo campo escalar para compensar. En el SM, el campo de Higgs es un doblete, y de los cuatro grados de libertad solo uno permanece como consecuencia de la ruptura de la simetría electrodébil, resultando en un bosón de Higgs. En el MSSM se necesitan dos dobletes de Higgs, $H_{u}=\left(H_{u}^{+}, H_{u}^{0}\right)$ y $H_{d}=\left(H_{d}^{0}, H_{d}^{-}\right)$. Esta notación tiene como ventaja que es fácil recordar el VEV de qué Higgos le da masa a cada tipo de quark. El escalar neutro que corresponde al bosón de Higgs del SM es una combinación lineal de $H_{u}^{0}$ y $H_{d}^{0}$. La nomenclatura usual para referirse a los supercompañeros de espín $\frac{1}{2}$ es agregar "ino" a la partícula del SM, por lo tanto los compañeros fermiónicos de los escalares de Higgs son denominados «higgsinos», y se denotan con $\widetilde{H_{u}}$ y $\widetilde{H_{d}}$.

Los bosones vectoriales del SM tienen que estar en supermultipletes de gauge. Sus supercompañeros fermiónicos son llamados «gauginos». Las interacciones de gauge de color de QCD son mediadas por el gluón, cuyo compañero supersimétrico de espín $\frac{1}{2}$ es el «gluino». Los gauginos supercompañeros de los bosones de gauge electrodébiles, luego de mezclarse con los supercompañeros de los bosones de Higgs, dan lugar a los autoestados de masa denominados «charginos» y «neutralinos». En la Tabla 1.3 se puede ver el espectro completo del MSSM.

\begin{tabular}{ccc}
\hline Supermultiplete & Bosón & Fermión \\
\hline gluon, gluino & $g$ & $\widetilde{g}$ \\
\hline W, wino & $W^{ \pm}, W^{0}$ & $\widetilde{W}^{ \pm}, \widetilde{W}^{0}$ \\
B, bino & $B$ & $\widetilde{B}$ \\
\hline sleptón, leptón & $(\widetilde{\nu}, \widetilde{e})_{L}$ & $(\nu, e)_{L}$ \\
$(\times 3$ generaciones $)$ & $e_{\mathrm{R}}$ & $e_{\mathrm{R}}$ \\
\hline squark, quark & $\left(\widetilde{u}_{\mathrm{L}}, \widetilde{d}_{\mathrm{L}}\right)$ & $\left(u_{\mathrm{L}}, d_{\mathrm{L}}\right)$ \\
$(\times 3$ generaciones $)$ & $\widetilde{u}_{\mathrm{R}}$ & $u_{\mathrm{R}}$ \\
& $\widetilde{d}_{\mathrm{R}}$ & $d_{\mathrm{R}}$ \\
\hline Higgs, higgsinos & $\left(H_{d}^{0}, H_{d}^{-}\right)$ & $\left(\widetilde{H}_{d}^{0}, \widetilde{H}_{d}^{-}\right)$ \\
& $\left(H_{u}^{+}, H_{u}^{0}\right)$ & $\left(\widetilde{H}_{u}^{+}, \widetilde{H}_{u}^{0}\right)$ \\
\hline
\end{tabular}

Tabla 1.3: Supermultipletes quirales y de gauge del MSSM.

Los supermultipletes quirales y de gauge de la Tabla 1.3 conforman el contenido de partículas del MSSM. Desde el punto de vista experimental, ninguna de las compañeras supersimétricas de las partículas del SM han sido observadas hasta el momento. Si una teoría es invariante bajo transformaciones supersimétricas, las partículas y sus correspondientes supercompañeras deben tener masas idénticas. Es decir, si la supersimetría no estuviera rota, deberían existir selectrones con una masa igual a $m_{e} \sim 0.511 \mathrm{MeV}$, y lo mismo para los demás sleptones y squarks. Y también deberían existir los gluinos y fotinos sin masa. El hecho de que no hayan sido descubiertas hasta el momento es motivo evidente de que SUSY es una simetría que está rota en el estado de vacío elegido por la naturaleza.

Pero una de las principales motivaciones para introducir esta nueva simetría fue que las divergencias cuadráticas en el cuadrado de las masas escalares podían anularse a todo orden en teoría de 
perturbaciones, y eso queda garantizado solo si esta simetría no está rota. Aunque no se conoce el mecanismo de ruptura de SUSY, este debe ser implementado de forma de que SUSY todavía pueda proveer la solución al problema de jerarquía incluso en presencia del rompimiento de esta. Para ello, las relaciones entre los acoplamientos adimensionales de la teoría antes del rompimiento deben mantenerse. Es por esta razón que el rompimiento de la supersimetría debe ser «suave», y el lagrangiano efectivo del MSSM tiene que poder ser escrito como:

$$
\mathcal{L}=\mathcal{L}_{\mathrm{SUSY}}+\mathcal{L}_{\text {soft }}
$$

donde $\mathcal{L}_{\text {SUSY }}$ contiene todas las interacciones de gauge y de Yukawa y preserva la invariancia supersimétrica. El conjunto de parámetros que aparecen en el lagrangiano $\mathcal{L}_{\mathrm{SUSY}}$ son:

- las constantes de acoplamiento de gauge $g_{s}, g$ y $g^{\prime}$ correspondientes a los grupos de gauge $\mathrm{SU}(3)_{\mathrm{C}}$, $\mathrm{SU}(2)_{\mathrm{L}}$ y $\mathrm{U}(1)_{\mathrm{Y}}$, respectivamente

- los acoplamientos de Yukawa que describen las interacciones entre fermiones y bosones de Higgs

- el parámetro de masa del campo de Higgs $\mu$.

El lagrangiano que rompe SUSY, $\mathcal{L}_{\text {soft }}$, no está completamente determinado y su forma explícita así como el conjunto de parámetros involucrados dependen del mecanismo particular de ruptura de SUSY. Debido a que este mecanismo es desconocido, se puede suponer un conjunto de términos de ruptura de la forma más general posible, sin indagar en sus orígenes, que se fijan solo pidiendo la invariancia frente $\mathrm{SU}(3)_{\mathrm{C}} \times \mathrm{SU}(2)_{\mathrm{L}} \times \mathrm{U}(1)_{\mathrm{Y}}, \mathrm{y}$ que sean suaves a fin de mantener la cancelación de las divergencias cuadráticas. Estos términos soft proveen exitosamente las masas de las partículas supersimétricas, a fin de que sean más pesadas que sus correspondientes compañeras del SM, y la ruptura espontánea de la simetría electrodébil requerida a bajas energías necesaria para explicar la generación de las masas de las partículas del SM. Si la escala de masa más grande asociada con los términos soft se llama $m_{\text {soft }}$, las correcciones adicionales no supersimétricas al cuadrado de la masa escalar del Higgs debe anularse en el límite $m_{\text {soft }} \rightarrow 0$.

Debido a que la diferencia de masas entre las partículas conocidas del SM y sus supercompañeras son determinadas por los parámetros $m_{\text {soft }}$ que aparecen en $\mathcal{L}_{\text {soft }}$, las masas de las partículas supersimétricas no pueden ser demasiado grandes. De otra forma se perdería la solución al problema de jerarquía. Pero por otro lado, también existe una razón por la cual las partículas supersimétricas deben ser lo suficientemente pesadas para no haber sido descubiertas hasta ahora. Todas las partículas del MSSM que han sido observadas tienen algo en común: deberían no tener masa en ausencia del rompimiento de la simetría electrodébil. En particular, las masas de los bosones $W^{ \pm}, Z^{0}$ y todos los quarks y leptones son iguales al producto de constantes de acoplamiento adimensionales por el $\langle H\rangle \sim 174 \mathrm{GeV}$, mientras que el fotón y el gluón necesitan ser no masivos por la invariancia de gauge electromagnética y de QCD. Por el contrario, todas las partículas del MSSM no descubiertas tienen la propiedad contraria. Cada una de ellas puede tener un término de masa en el lagrangiano en ausencia del rompimiento de la simetría electrodébil.

El conjunto de nuevos parámetros que aparece en el lagrangiano de ruptura de SUSY incluye [33,50]:

- Las masas soft del sector de Higgs, $m_{1}, m_{2}$ y $m_{12}$, donde $m_{12}^{2} \equiv B \mu, \mu$ es el parámetro introducido anteriormente y $B$ es el parámetro de ruptura de SUSY.

- Las masas soft de los squarks y sleptones de cada generación: $m_{\widetilde{Q}}, m_{\widetilde{U}}, m_{\widetilde{D}}, m_{\widetilde{L}}$ y $m_{\widetilde{E}}$.

- Los acoplamientos trilineales de squarks y sleptones $A_{q}$ y $A_{l}$. 
- Las masas soft de los gauginos, $M_{3}, M_{2}$ y $M_{1}$, asociadas a los grupos de gauge del SM SU $(3)_{\mathrm{C}}$, $\mathrm{SU}(2)_{\mathbf{L}}$ y $\mathbf{U}(1)_{\mathrm{Y}}$, respectivamente.

En un modelo con una sola generación de quarks, leptones y sus supercompañeros, el MSSM tendría 14 nuevos parámetros. En el modelo completo de tres generaciones, el número de nuevos parámetros es sustancialmente mayor ya que los parámetros de masa de los squarks y sleptones y los parámetros $A$ serán matrices de $3 \times 3$, y la posibilidad de mezcla entre generaciones puede llevar a complicaciones adicionales. Sin embargo, no todos estos parámetros son físicos. Algunos de ellos pueden ser eliminados expresando los autoestados de interacción en términos de los autoestados de masa, con la apropiada redefinición de los campos del MSSM para remover los grados de libertad no físicos. El análisis de la referencia [51] muestra que el MSSM posee 124 parámetros independientes. De estos, 18 corresponden a los parámetros del SM, uno corresponde al sector de Higgs (el análogo a la masa del Higgs del SM), y 105 son nuevos parámetros del modelo.

En un tratamiento fenomenológico completo todos los parámetros del MSSM deberían dejarse libres y determinarse a partir de los datos observados, y luego de que los parámetros hayan sido medidos, se podría intentar extraer información de la física subyacente que está asociada con escalas de energía mayores a la de los experimentos. Sin embargo, realizar predicciones y análisis fenomenológicos con esta cantidad de parámetros es impracticable, por lo cual es necesario realizar suposiciones para reducir los grados de libertad. Es debido a este motivo que no existe una definición precisa del MSSM y es importante conocer cuales son las suposiciones que se han hecho cuando se realiza un determinado análisis.

Hay un propiedad adicional del MSSM que hace que sea menos restrictivo que el SM. Es bien sabido que si se impone la invariancia del lagrangiano del SM bajo la simetría $\mathrm{SU}(3)_{\mathrm{C}} \times \mathrm{SU}(2)_{\mathrm{L}} \times \mathrm{U}(1)_{\mathrm{Y}}$, se encuentra que todos los términos de dimensión 4 o menor preservan automáticamente el número bariónico $B$ y leptónico $L$. Este no es el caso del MSSM, en el que la forma general del superpotencial tiene como inconveniente que el número leptónico y bariónico no es una cantidad conservada. Esto implica que el protón puede decaer a través del intercambio del compañero escalar del quark $d$, en clara contradicción con la evidencia experimental que establece un límite superior en la vida media del protón de $>10^{30}$ años a $90 \%$ CL [33].

A fin de evitar este problema, la estrategia más comúnmente utilizada consiste en introducir una nueva simetría. A esta simetría se la conoce como paridad-R y se define como $P_{R}=(-1)^{3(B-L)+2 s}$, donde $B$ y $L$ son respectivamente el número bariónico y leptónico, y $s$ es el espín de la partícula. Las partículas supersimétricas tienen $P_{R}=-1$, mientras que las partículas del SM tienen $P_{R}=+1$.

Si la paridad-R es exactamente conservada, no puede haber mezcla entre las spartículas y las partículas del SM. Cada vértice de interacción en la teoría contiene entonces un número par de spartículas, lo que conlleva a algunas consecuencias fenomenológicas de extrema importancia:

- En experimentos de colisionadores, las partículas supersimétricas pueden solo ser producidas en número par, en general de a dos.

- La partícula supersimétrica más liviana, llamada LSP, es estable y no tiene ninguna partícula con $P_{R}=-1$ a la cual decaer. Si la LSP es eléctricamente neutra, interactúa solo de forma débil con la materia ordinaria, y por lo tanto resulta en un candidato muy atractivo para la materia oscura no bariónica que es requerida por la cosmología. Desde el punto de vista experimental la LSP solo será visible a través de la energía faltante dejada en el detector.

- Cada partícula supersimétrica que no sea la LSP debe decaer eventualmente en un estado que contenga un número impar de LSP (en general una). 


\section{El espectro de masas del MSSM}

Una característica importante del MSSM es que las nuevas partículas, que están listadas en la Tabla 1.3, no son necesariamente autoestados de masa de la teoría. Después del rompimiento de la simetría electrodébil y de la supersimetría, puede haber mezcla entre los gauginos y higgsinos, y entre los squarks y sleptones y los Higgs escalares que tienen la misma carga eléctrica.

En el MSSM, la descripción del rompimiento de la simetría electrodébil es un poco más complicada que en el SM debido al hecho de que hay dos dobletes complejos de Higgs $H_{u}$ y $H_{d}$ en vez de solo uno. Examinar los distintos autovalores de masa de la teoría, no es un análisis trivial, ya que cualquier conjunto de partículas con los mismos números cuánticos puede mezclarse. En las siguientes secciones se analizan los distintos sectores del modelo.

- Squarks y sleptones

Cualquier par de escalares con la misma carga eléctrica, paridad-R y color pueden mezclarse entre ellos. Después del agregado de los términos del rompimiento soft de la supersimetría, los autoestados de masa de los squarks y sleptones del MSSM pueden obtenerse diagonalizando tres matrices de $6 \times 6$ para squarks up, down y sleptones cargados, y una matriz adicional de $3 \times 3$ para los sneutrinos.

\section{- Neutralinos y Charginos}

Los higgsinos y los gauginos electrodébiles se mezclan entre ellos debido al efecto del rompimiento de la simetría electrodébil. Los higgsinos neutros $\left(\widetilde{H}_{u}^{0}\right.$ y $\left.\widetilde{H}_{d}^{0}\right)$ y los gauginos neutros $\left(\widetilde{B}, \widetilde{W}^{0}\right)$ se combinan para formar cuatro autoestados de masa llamados «neutralinos». Los higgsinos cargados $\left(\widetilde{H}_{u}^{+}\right.$y $\left.\widetilde{H}_{d}^{-}\right)$y los winos $\left(\widetilde{W}^{+}\right.$y $\left.\widetilde{W}^{-}\right)$se mezclan para formar dos autoestados de masa con carga \pm 1 llamados «charginos».

La mezcla de los gauginos y higgsinos cargados está descripta a orden árbol por la matriz de $2 \times 2$ :

$$
M_{C}=\left(\begin{array}{cc}
M_{2} & \sqrt{2} m_{W} \operatorname{sen} \beta \\
\sqrt{2} m_{W} \cos \beta & \mu
\end{array}\right)
$$

la cual se debe diagonalizar para determinar los estados físicos y las masas de los charginos. Estos dos autoestados de masa se indican con $\widetilde{\chi}_{1}^{ \pm}$y $\widetilde{\chi}_{2}^{ \pm}$y su masa está dada por:

$$
M_{\tilde{\chi}_{1,2}}^{2}=\frac{1}{2}\left\{M_{2}^{2}+\mu^{2}+2 m_{W}^{2} \mp\left[\left(M_{2}^{2}-\mu^{2}\right)^{2}+4 m_{W}^{4} \cos ^{2} 2 \beta+4 m_{W}^{2}\left(M_{2}^{2}+\mu^{2}+2 M_{2} \mu \operatorname{sen} 2 \beta\right)\right]^{1 / 2}\right\}
$$

En el caso de los neutralinos la matriz de masa es:

$$
M_{N}=\left(\begin{array}{cccc}
M_{1} & 0 & -\cos \beta \operatorname{sen} \theta_{W} m_{Z} & \operatorname{sen} \beta \operatorname{sen} \theta_{W} m_{Z} \\
0 & M_{2} & \cos \beta \cos \theta_{W} m_{Z} & -\operatorname{sen} \beta \cos \theta_{W} m_{Z} \\
-\cos \beta \operatorname{sen} \theta_{W} m_{Z} & \cos \beta \cos \theta_{W} m_{Z} & 0 & -\mu \\
\operatorname{sen} \beta \operatorname{sen} \theta_{W} m_{Z} & -\operatorname{sen} \beta \cos \theta_{W} m_{Z} & -\mu & 0
\end{array}\right)
$$

Para el caso de los neutralinos se utiliza la siguiente notación $\tilde{\chi}_{i}^{0}(i=1,2,3,4)$ y al igual que en 
el caso de los charginos son ordenados de forma ascendente según su masa (es decir, el $i=1$ es el más liviano).

Si un estado de chargino o neutralino se aproxima a un estado particular de gaugino o higgsino, es conveniente usar la nomenclatura correspondiente. Si $M_{1}$ y $M_{2}$ son pequeñas comparadas a $m_{Z}$ y $|\mu|$, el neutralino más liviano va a ser puramente fotino. Si $M_{1}$ y $m_{Z}$ son pequeños comparados a $M_{2}$ y $|\mu|$, entonces el neutralino más liviano va a ser puramente bino. Si $M_{2}$ y $m_{Z}$ son pequeñas comparadas con $M_{1} \mathrm{y}|\mu|$, el par de charginos más liviano y neutralino va a constituir un triplete de winos puro degenerado en masas. Finalmente, si $|\mu|$ y $m_{Z}$ son chicos comparados con $M_{1}$ y $M_{2}$, el neutralino más liviano va a ser un higgsino puro. Cada unos de los casos anteriores va a llevar a un fenomenología muy distinta.

- Gluinos

Como el gluino es un fermión de color con ocho componentes, no puede mezclarse con ninguna otra partícula del MSSM (aunque haya violación de paridad-R). Esto significa que el único parámetro de masa soft que entra en el cálculo de la masa de los gluinos es, a orden árbol, $M_{3}$, siendo $m_{\widetilde{g}}=M_{3}$.

- Sector de Higgs

Los campos escalares de Higgs en el MSSM consisten en dos dobletes de $\mathrm{SU}(2)_{\mathrm{L}}$ complejos $H_{u}$ y $H_{d}$, con 8 grados de libertad escalares. Después del rompimiento de la simetría electrodébil, tres de estos son bosones de Nambu-Goldstone, que se convierten en los modos longitudinales de los bosones vectoriales $Z$ y $W^{ \pm}$. Los restantes cinco grados de libertad producen los cinco bosones de Higgs físicos del modelo: el par de bosones de Higgs cargados $H^{ \pm}$; un bosón de Higgs neutral CP-impar $A^{0}$; y los bosones de Higgs neutral CP-par, $H^{0}, h^{0}$, donde por convención $h^{0}$ es más liviano que $H^{0}$.

La particularidad del sector de bosones de Higgs del MSSM es que las masas y mezclas de los bosones de Higgs están determinadas, a orden árbol y debido a la supersimetría, por solo dos parámetros que usualmente se eligen $\operatorname{como} m_{A_{0}} \mathrm{y} \tan \beta$.

\section{Decaimiento de spartículas}

- Sleptones y squarks

Los sleptones puede decaer en un leptón y un chargino/neutralino,

$$
\begin{aligned}
& \tilde{\ell} \rightarrow \ell \widetilde{\chi}^{0}, \nu \widetilde{\chi}^{ \pm} \\
& \widetilde{\nu} \rightarrow \nu \tilde{\chi}^{0}, \ell \widetilde{\chi}^{ \pm}
\end{aligned}
$$

Como los sleptones derechos no se acoplan al gaugino de $\mathrm{SU}(2)_{\mathrm{L}}$, comúnmente prefieren el decaimiento directo a $\ell \widetilde{\chi}_{1}^{0}$.

Los squarks pueden tener decaimiento a dos cuerpos en un squark-gluino si es cinemáticamente permitido, porque tienen acoplamiento fuerte. Por otro lado, los squarks pueden decaer en un quark y un neutralino/chargino.

$$
\widetilde{q} \rightarrow q \widetilde{g}, q \widetilde{\chi}^{0}, q^{i} \widetilde{\chi}^{ \pm}
$$


- Neutralinos y charginos

Como cada neutralino y chargino contiene al menos una mezcla de los gauginos electrodébiles $\widetilde{B}$, $\widetilde{W}^{0}$ o $\widetilde{W}^{ \pm}$, heredan los acoplamientos de intensidad electrodébil a los pares de fermiones escalares. Si los sleptones o squarks son lo suficientemente livianos, un neutralino o chargino va a decaer a un par leptón+slepton o quark+squark. También pueden decaer en un neutralino o chargino más liviano y un Higgs escalar o un bosón de gauge electrodébil. Los posibles decaimientos son

$$
\begin{aligned}
& \widetilde{\chi}_{i}^{0} \rightarrow Z \widetilde{\chi}_{j}^{0}, \quad W \widetilde{\chi}_{j}^{ \pm}, \quad h^{0} \widetilde{\chi}_{j}^{0}, \quad \ell \widetilde{\ell}, \quad \nu \widetilde{\nu}, \quad\left[A^{0} \widetilde{\chi}_{j}^{0}, \quad H^{0} \widetilde{\chi}_{j}^{0}, \quad H^{ \pm} \widetilde{\chi}_{j}^{\mp}, \quad q \widetilde{q}\right], \\
& \widetilde{\chi}_{i}^{ \pm} \rightarrow W^{ \pm} \widetilde{\chi}_{j}^{\mp}, \quad Z \widetilde{\chi}_{1}^{ \pm}, \quad h^{0} \widetilde{\chi}_{1}^{ \pm}, \quad \ell \widetilde{\nu}, \quad \nu \widetilde{\ell}, \quad\left[A^{0} \widetilde{\chi}_{1}^{ \pm}, \quad H^{0} \widetilde{\chi}_{1}^{ \pm}, \quad H^{ \pm} \widetilde{\chi}_{j}^{0}, \quad q \widetilde{q}^{\prime}\right]
\end{aligned}
$$

donde los estados finales entre corchetes son los cinemáticamente menos probables.

\section{- Gluinos}

El gluino solo puede decaer a través de un squark, ya sea on-shell o virtual. Si el decaimiento a dos cuerpos está abierto, este va a dominar debido al acoplamiento gluino-quark-squark es fuerte. En el caso de que todos los squarks sean más pesados que el gluino, este va a decaer solo vía squarks virtuales.

$$
\begin{aligned}
& \widetilde{g} \rightarrow q \widetilde{q}, \\
& \widetilde{g} \rightarrow q \tilde{\chi}^{0}, \quad q q^{\prime} \tilde{\chi}^{ \pm}
\end{aligned}
$$

\subsubsection{Origen de la ruptura de SUSY. Modelos GMSB}

Como fue mencionado en la sección anterior, debido a que no se ha observado ninguna de las partículas supersimétricas predichas, si existe SUSY, debe estar rota, y para mantener la solución al problema de jerarquía incluso en presencia del rompimiento de ésta, el rompimiento debe ser suave, incluyendo términos soft al lagrangiano. En el MSSM, el rompimiento de SUSY es introducido explícitamente. El rompimiento de una simetría global siempre implica un modo no masivo de NambuGoldstone con los mismos números cuánticos que el generador de la simetría rota. En el caso de la supersimetría global, el generador es la carga fermiónica $Q_{\alpha}$, por lo tanto la partícula de NambuGoldstone tiene que ser un fermión de Weyl no masivo neutro, llamado goldstino. Es claro ahora que el rompimiento espontáneo de la supersimetría requiere la extensión del MSSM. El rompimiento espontáneo de SUSY tiene que ocurrir en un «sector oculto» de partículas que no tienen acoplamientos directos con los supermultipletes quirales («sector visible») del MSSM. Sin embargo, estos dos sectores comparten algunas interacciones que son las responsables de mediar el rompimiento de la supersimetría desde el sector oculto al visible, resultando en los términos soft del MSSM (ver Figura 1.5).

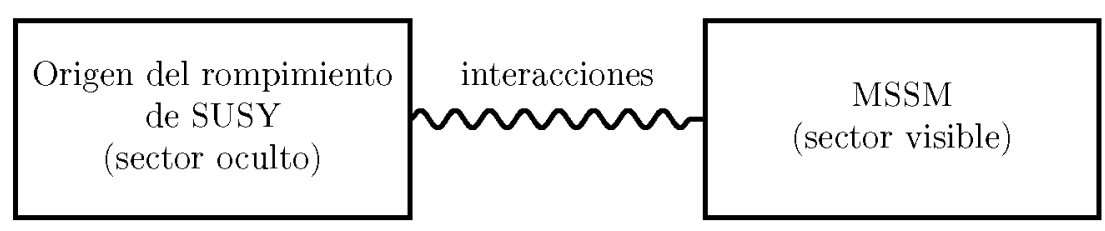

Figura 1.5: Esquema de la supuesta estructura del rompimiento de supersimetría. 
Existen diferentes propuestas acerca de las interacciones mediadores. Una de ellas (e históricamente la más popular) es que estas interacciones son gravitacionales (mSUGRA).

Una segunda posibilidad es que estas interacciones mediadores sean las interacciones de gauge electrodébiles y QCD ordinarias. En estos escenarios donde el rompimiento de supersimetría está mediado por campos de gauge (Gauge-mediated supersymmetry breaking ó GMSB) [52], los términos soft del MSSM provienen de diagramas a un loop que involucran algunas partículas mensajeras.

Si se tiene en cuenta a la gravedad, SUSY tiene que ser promovida a una simetría local. Esta teoría supersimétrica local es llamada «supergravedad». Esto unifica necesariamente las simetrías espaciotemporales ordinarias de la Relatividad General con las transformaciones locales supersimétricas. En esta teoría, el gravitón de espín 2, tiene un supercompañero fermión de espín $\frac{3}{2}$ llamado «gravitino». Mientras la supersimetría no esté rota, el gravitón y el gravitino son no masivos con dos estados de helicidad de espín. Una vez que SUSY es espontáneamente rota, el gravitino adquiere una masa absorbiendo el goldstino, que se convierte en sus componentes longitudinales (helicidad $\pm \frac{1}{2}$ ). Este mecanismo es llamado super-Higgs, y es el análogo al mecanismo de Higgs ordinario de las teorías de gauge, donde los bosones de gauge $W^{ \pm}$y $Z^{0}$ en el Modelo Estándar adquieren masa absorbiendo los bosones de Nambu-Goldstone asociados con la invariancia de gauge electrodébil espontáneamente rota. La masa del gravitino es tradicionalmente llamada $m_{3 / 2}$, y puede ser estimada como $m_{3 / 2} \sim\langle F\rangle / M_{P}$, donde $F$ está relacionada a la escala del rompimiento de SUSY. Esto implica distintos valores esperados para la masa del gravitino dependiendo el modelo de mediadores propuesto.

En modelos de mediación por gravedad, la masa del gravitino es comparable a la masa de las partículas del MSSM, por lo tanto es esperado que sea de al menos el orden de $100 \mathrm{GeV}$. En contraste, los modelos GMSB predicen un gravitino mucho más liviano que las spartículas del MSSM. En este caso, el gravitino es la LSP, y todas las spartículas del MSSM van a decaer eventualmente en un estado final que incluye al gravitino.

En los modelos de rompimiento de la supersimetría mediado por campos de gauge o GMSB, las interacciones ordinarias de gauge son las responsables de la aparición del rompimiento soft de la supersimetría en el MSSM. La mediación por campos de gauge es una de las maneras más simples y robustas para transmitir el rompimiento de SUSY al MSSM. A pesar de su simpleza, existe un gran número de modelos de GMSB. Para poder hacer un análisis fenomenológico dentro del marco de GMSB de una forma no tan dependiente del modelo, pero con un correcta base teórica, en [25] se propone un marco lo más general posible para describir los efectos de un sector oculto arbitrario, definiendo al mecanismo de mediación por campos de gauge como: en el límite en que las constantes de acoplamiento del MSSM $\alpha_{i} \rightarrow 0$, la teoría se desacopla en el MSSM y un sector oculto separado que rompe SUSY.

A este marco general se lo conoce como General Gauge Mediation o GGM. El conjunto de parámetros independientes de GGM está compuesto por las tres masas complejas de gauginos $M_{1}, M_{2}$ y $M_{3}$, y tres parámetros reales que controlan las masas de los cinco sfermiones $m_{Q, U, D, L, E}^{2}$. Además, los términos trilineales $A$ siempre son pequeños.

Estos modelos ofrecen una rica variedad de estados finales en colisionadores [53]. La característica más destacable de estos modelos es el gravitino liviano $m_{3 / 2} \ll M_{W}$. La masa del gravitino en modelos GGM es típicamente del orden del eV (en algunos casos puede llegar al GeV), lo cual implica que el $\widetilde{G}$ es la LSP. Esto asegura que los efectos de mediación por campos de gauge dominen por sobre la mediación por gravedad.

Uno de los aspectos teóricos interesantes de SUSY es que si esta simetría es realizada como una simetría local, incorpora de forma necesaria y automática la gravedad. La masa del gravitino está relacionada con la escala del rompimiento de SUSY por, 


$$
m_{\bar{G}}=\frac{\Lambda_{\mathrm{SUSY}}^{2}}{\sqrt{3} M_{P}}
$$

La escala $\Lambda_{\mathrm{SUsY}}$ tiene que ser al menos la masa de las partículas supersimétricas más pesadas. Si consideramos que $\Lambda_{\mathrm{SUSY}}>1 \mathrm{TeV}$ entonces $m_{\widetilde{G}}>10^{-4} \mathrm{eV}$. Y por otro lado, los límites cosmológicos $[54,55]$ bajo ciertas condiciones, ponen un límite superior $m_{\widetilde{G}}<10^{4} \mathrm{eV}$.

El hecho de que la LSP sea siempre el gravitino, determina que la partícula más liviana del MSSM es entonces la NLSP, y siempre decae a un gravitino y su compañero del SM. Como este decaimiento está altamente suprimido por la escala de rompimiento de SUSY, la NLSP puede decaer de forma inmediata ( prompt) o luego de haber atravesado una cierta distancia en el detector. En lo que sigue solo se consideran los decaimientos prompt. Esta supresión de la tasa de decaimiento también significa que todas las spartículas pesadas decaen pasando por la NLSP antes de decaer en un gravitino. Es por este motivo que la naturaleza de la NLSP es el aspecto más importante del espectro de GMSB para los estados finales en colisionadores.

La topología típica de un evento GGM está ilustrada en la Figura 1.6, en la cual se pueden observar varias características importantes. El gravitino siempre va a escapar del detector, dando lugar a una cantidad significativa de energía faltante. Mientras tanto, el compañero del SM va a tender a ser central y energético (suponiendo que la NLSP decae dentro del detector), ya que es en general mucho más liviano que la NLSP. Dado que en cada evento SUSY se producen dos NLSPs, es claro que los estados finales en un colisionador van a estar determinadas por la naturaleza de la NLSP.

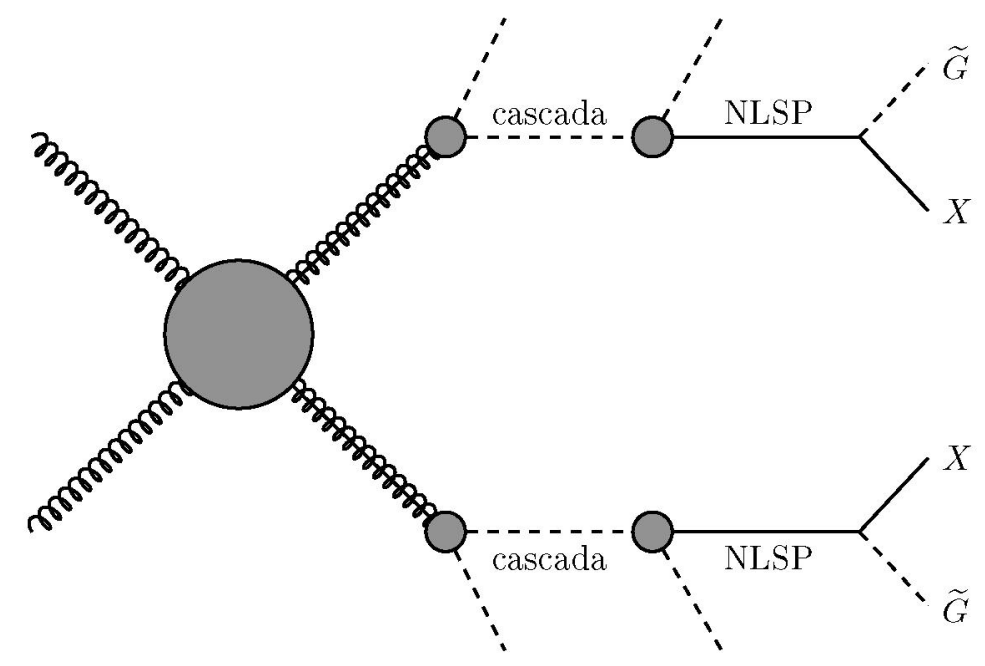

Figura 1.6: Esquema de la topología típica de un evento GGM en un colisionador de hadrones. En la interacción fuerte se produce un par de gluinos que luego decaen en forma de cascada, hasta llegar a la NLSP, que decae en un gravitino $\widetilde{G}$ y su compañero del SM $(X)$. Los gravitinos escapan el detector dejando un gran cantidad de energía faltante.

\section{Neutralino NLSP}

Como se ha mencionado, la segunda partícula supersimétrica más liviana (NLSP) juega un rol fundamental en la fenomenología de los modelos GGM. Suponiendo que se conserva la paridad-R, todas las partículas supersimétricas van a decaer rápidamente en una cascada hasta la NLSP, y esta va a decaer en un gravitino y una partícula del SM. Por este motivo, la naturaleza de la NLSP determina los diferentes estados finales en los colisionadores. En principio la NLSP puede ser cualquier partícula 
del MSSM dependiendo de la elección de los parámetros del modelo. Para la mayor parte del espacio de parámetros será el neutralino o el stau, y para algunas regiones muy restrictivas, el sneutrino [52]. Las partículas, que aunque no son NLSP, tienen un decaimiento dominante en su compañero supersimétrico y el $\widetilde{G}$ se denominan co-NLSP.

Todos estos casos corresponden a una fenomenología completamente diferente en los experimentos de altas energías. Para el trabajo de esta tesis resulta de interés el caso en que la NLSP es el neutralino más liviano, ya que en ese caso son comunes los estados finales con fotones.

Como se detalla en la sección 1.2.1, los autoestados de masa de los neutralinos dependen de cuatro parámetros: $M_{1}, M_{2}, \mu$ y $\tan \beta$. Dependiendo de los valores de estos cuatro parámetros, el decaimiento dominante será distinto. Como el espacio de parámetros del neutralino es de cuatro dimensiones, resulta difícil hacer un estudio detallado de la fenomenología asociada. En general se estudian algunos límites simplificados, donde el neutralino es básicamente uno de los autoestados de gauge puro, es decir, es puramente bino, wino o higgsino.

El neutralino NLSP decae a $X+\widetilde{G}$ donde $X=\gamma, Z, h, y$ los distintos autoestados de gauge se caracterizan por tener distintas tasas de decaimiento (BR) a los diferentes $X$ [56]. Los binos decaen a fotones con un $\mathrm{BR} \sim \cos ^{2} \theta_{W}$, con una componente menor a $Z$ 's, con $\mathrm{BR} \sim \sin ^{2} \theta_{W}$ (ver figura Figura 1.7). Por otro lado estos BR se intercambian en el caso de que la NLSP sea el wino neutro, que decae mayormente a $Z$ 's. Si la NLSP es higgsino, este decae en forma dominante a $Z$ ó $h$, con BR que depende en el valor de $\tan \beta$ y del signo de $\mu$. Hay tres casos: (i) el decaimiento del higgsino es preferentemente a $Z$ para bajo $\tan \beta$ y $\mu$ positivo, (ii) mayormente a $h$ para bajo tan $\beta$ y $\mu$ negativo, y (iii) una mezcla de $Z$ y $h$ a moderado y alto $\tan \beta$.
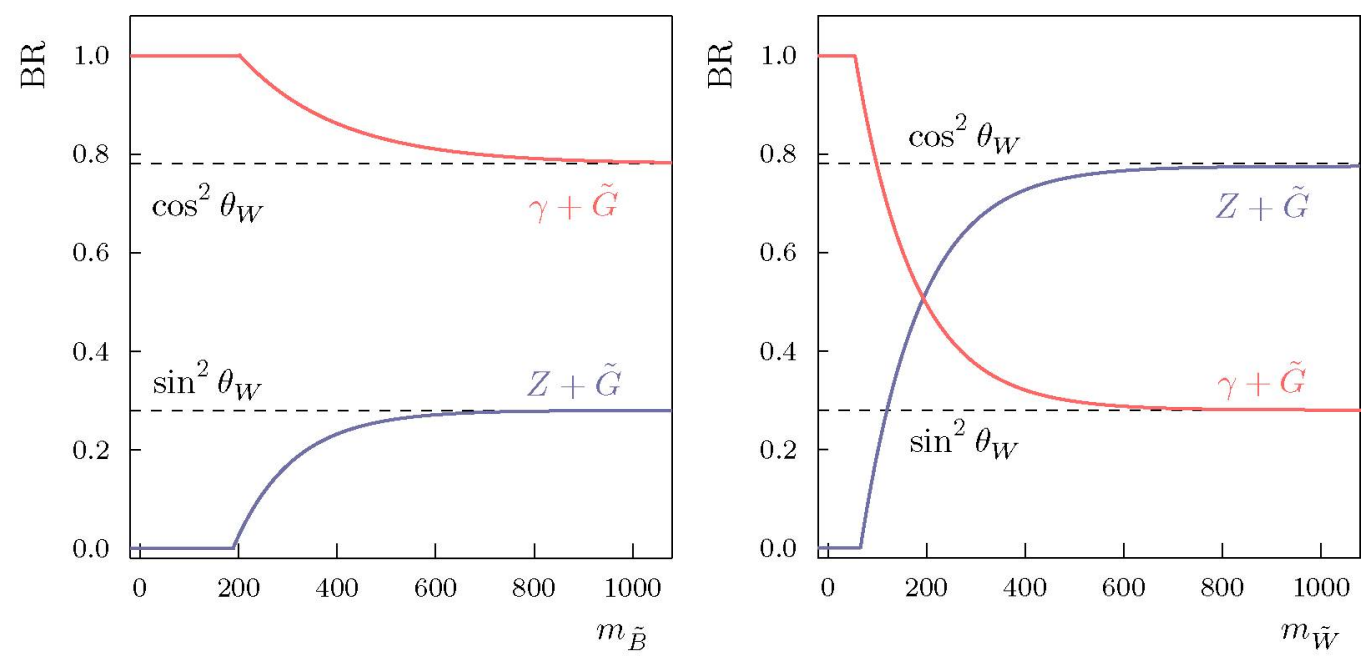

Figura 1.7: Tasas de decaimiento del neutralino más liviano a gravitino más fotones o bosones $Z$ en función de la masa del mismo. En la izquierda el caso en que el neutralino es puramente bino, y a la derecha puramente wino.

Cuando la NLSP es mayormente wino, hay una muy pequeña degeneración entre el wino neutro y el cargado. Cuando esto pasa, el decaimiento de tres cuerpos al wino neutro comienza a ser excluido, y el wino cargado prefiere decaer directamente a $W^{ \pm}$y un gravitino. En otras palabras, el wino neutro y el wino cargado se vuelven co-NLSPs, y los estados finales van a contener W's, $Z$ 's y fotones.

Como en cada evento de SUSY hay un par de neutralinos, los estados finales involucrarán una combinación de pares de $\gamma+\widetilde{G}, Z+\widetilde{G}$ y $h+\widetilde{G}$. Adicionalmente, si hay una diferencia chica entre la 
masa del neutralino y del chargino más liviano, puede haber cadenas de decaimiento que terminen en $W^{ \pm}+\widetilde{G}$.

El escenario más estudiado y más buscado es cuando la NLSP es un neutralino puramente bino. En este caso el estado final consiste en $\gamma \gamma+E_{\mathrm{T}}^{\text {miss }}$. Sin embargo en el caso más general de neutralino NLSP, aparecen muchas otros canales menos explorados que involucran leptones, $Z, W$, jets y higgses de alto $p_{\mathrm{T}}$ y energía faltante.

En el caso en el cual el neutralino es una mezcla mayoritaria de bino y higgsino, el estado final dependerá del parámetro de masa del higgsino $\mu$. Si $\mu<0$, el estado final de la cascada va a tener una gran contribución de $\widetilde{\chi}_{1}^{0} \rightarrow h \widetilde{G}$ donde el Higgs decaerá mayormente como $h \rightarrow b \bar{b}$, dejando un estado final con un fotón, $b$-jets y $E_{\mathrm{T}}^{\mathrm{miss}}$. En el escenario donde $\mu>0$ el decaimiento dominante estará compartido entre $\widetilde{\chi}_{1}^{0} \rightarrow \gamma \widetilde{G}$ y $\widetilde{\chi}_{1}^{0} \rightarrow Z \widetilde{G}$, y el decaimiento a Higgs estará suprimido, dando como estado final un fotón, múltiples jets (incluyendo los dos del decaimiento del bosón $Z$ ) y $E_{\mathrm{T}}^{\text {miss }}$.

Esta tesis se centrará en este último caso. En el capítulo 6 se describirá en detalle el modelo de señal que motiva el estado final del presente análisis.

\subsubsection{Producción de partículas supersimétricas}

En los colisionadores hadrónicos, las partículas supersimétricas pueden ser producidas de a pares a partir de colisiones partón-partón de interacción electrodébil (ver Figura 1.8):

$$
\begin{aligned}
& q \bar{q} \rightarrow \tilde{\chi}^{+} \tilde{\chi}^{-}, \tilde{\chi}^{0} \widetilde{\chi}^{0} \\
& q \bar{q} \rightarrow \widetilde{\ell}_{i}^{+} \tilde{\ell}_{j}^{-}, \widetilde{\nu}_{\ell} \widetilde{\nu}_{\ell}^{*}
\end{aligned}
$$

y a partir de interacciones fuertes (ver Figura 1.9):

$$
\begin{aligned}
g g & \rightarrow \widetilde{g} \widetilde{g}, \widetilde{q}_{i}{\widetilde{q_{j}}}^{*}, \\
g q & \rightarrow \widetilde{g} \widetilde{q}_{i}, \\
q \bar{q} & \rightarrow \widetilde{g} \widetilde{g}, \widetilde{q_{i}}{\widetilde{q_{j}}}^{*}, \\
q q & \rightarrow \widetilde{q_{i}} \widetilde{q}_{j},
\end{aligned}
$$
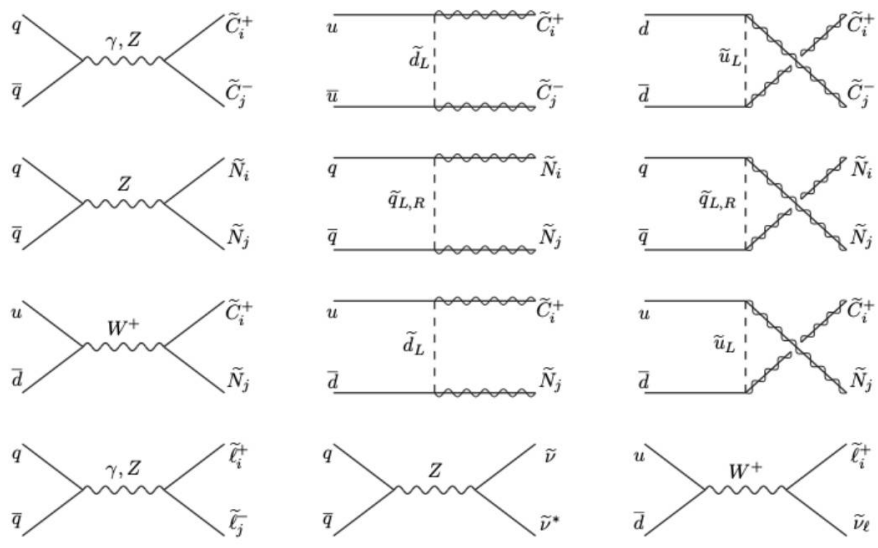

Figura 1.8: Diagramas de Feynman para la producción electrodébil de spartículas en colisionadores de hadrones vía aniquilación quark-antiquark. 

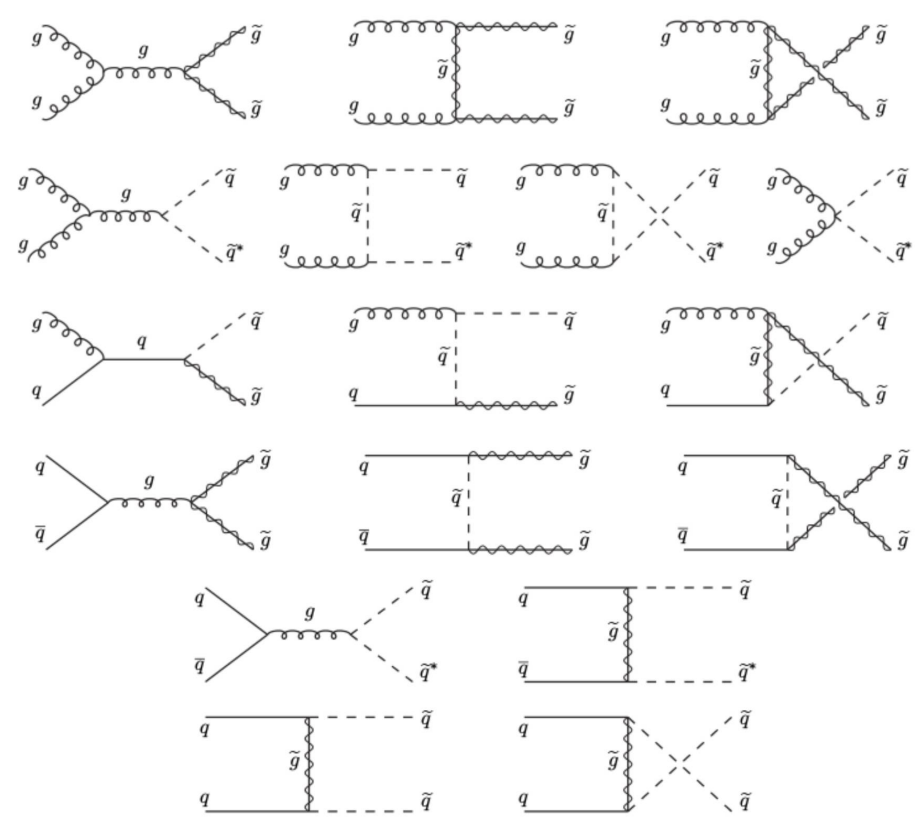

Figura 1.9: Diagramas de Feynman para la producción de gluinos y squarks en colisionadores de hadrones vía fusión gluón-gluón y gluón-squark, aniquilación quark-antiquark y dispersión quark-quark.

En la Figura 1.10 se puede ver la sección eficaz para la producción de squarks y gluinos en el LHC comparada para los distintos canales de producción. La producción por interacción fuerte es mucho mayor a la de spartículas que interactúan débilmente, y por esta razón la mayoría de las búsquedas se basan en la producción de gluinos y squarks.

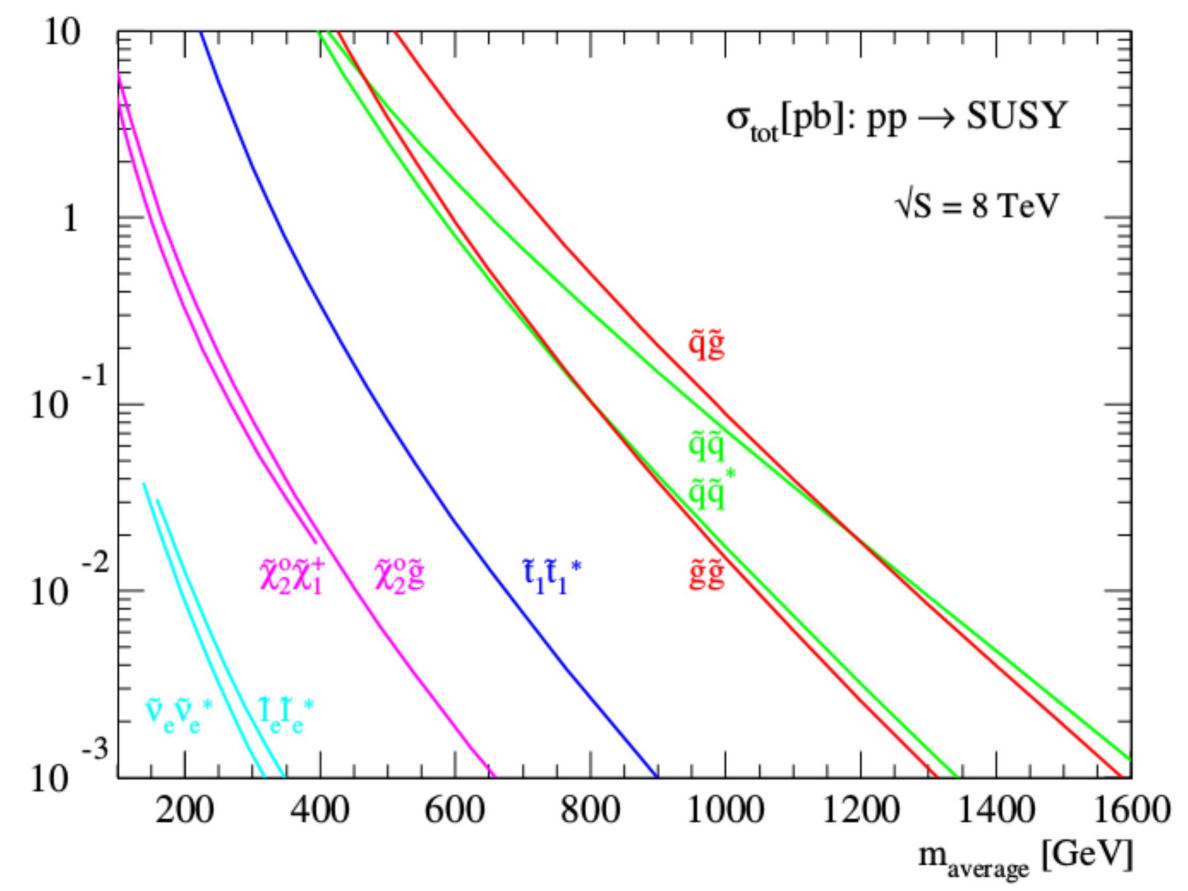

Figura 1.10: Sección eficaz de producción de partículas supersimétricas, como función de su masa, para los distintos canales de producción en el LHC con $\sqrt{s}=8 \mathrm{TeV}$ calculada a NLO usando Prospino [57]. 


\section{PARTE II \\ EXPERIMENTO}





\section{EL LHC Y EL DETECTOR ATLAS}

\subsection{LHC}

El Gran Colisionador de Hadrones (LHC, del inglés Large Hadron Collider) [1] es el acelerador de hadrones del Centro Europeo para la Investigación Nuclear (CERN), ubicado en la frontera entre Francia y Suiza. Posee una longitud de $27 \mathrm{~km}$ y fue construido en el mismo túnel en el que funcionaba el acelerador $e^{+} e^{-}$LEP [58] (entre 1989 y 2000), a una profundidad variable entre 50 y $174 \mathrm{~m}$ de la superficie.

El complejo de aceleradores del CERN (ver Figura 2.1) esta formado por una sucesión de aceleradores que culmina con el LHC, que es el último elemento en la cadena, y está diseñado para acelerar cada haz de protones a $7 \mathrm{TeV}$, alcanzando en las colisiones energías de centro de masa de $14 \mathrm{TeV}$ una luminosidad de $10^{34} \mathrm{~cm}^{-2} \mathrm{~s}^{-1}$. Los demás aceleradores en la cadena poseen además otros experimentos que utilizan los haces a menores energías.

El número de eventos de un dado proceso producidos en un colisionador, como el LHC, está dado por:

$$
N=L \sigma
$$

donde $\sigma$ es la sección eficaz del proceso físico y $L$ es la luminosidad integrada del acelerador.

La luminosidad instantánea es uno de los parámetros más importantes para caracterizar el funcionamiento del acelerador, definida como el número de partículas (protones o iones pesados en el caso del LHC) por unidad de tiempo y unidad de área, y puede calcularse mediante la relación:

$$
\mathcal{L}=f_{\text {rev }} n_{b} \frac{N_{1} N_{2}}{A}
$$

donde $f_{\mathrm{rev}}$ es la frecuencia de revolución $(\sim 11 \mathrm{kHz}), n_{b}$ es el número de bunches (paquetes de protones) por haz, $N_{i}$ es el número de partículas en cada bunch y $A$ es la sección efectiva del haz, que puede expresarse en término de los parámetros del acelerador como:

$$
A=\frac{4 \pi \epsilon_{n} \beta^{*}}{\gamma F}
$$

donde $\epsilon_{n}$ es la emitancia transversal normalizada (la dispersión transversal media de las partículas del haz en el espacio de coordenadas e impulsos), $\beta^{*}$ es la función de amplitud en el punto de interacción, relacionada al poder de focalización de los cuadrupolos), $\gamma$ es el factor relativista de Lorentz y $F$ es un factor de reducción geométrico, debido al ángulo de cruce de los haces en el punto de interacción.

El diseño del LHC contempla trenes de 2808 paquetes de $\sim 10^{11}$ protones cada uno, espaciados temporalmente en 25 ns. Para acelerar los haces de protones y mantenerlos en sus órbitas circulares el 
LHC cuenta con 1232 dipolos magnéticos superconductores que generan un campo magnético de $8.4 \mathrm{~T}$ enfriados a $1.9 \mathrm{~K}$. El sistema de focalización de los haces consiste de 392 cuadrupolos magnéticos que generan campos magnéticos de $6.8 \mathrm{~T}$. Los haces circulan en direcciones opuestas en cavidades de ultra alto vacío a presión de $10^{-10}$ torr.

Durante el año 2012, las colisiones se realizaron a $4 \mathrm{TeV}$ por haz $(\sqrt{s}=8 \mathrm{TeV})$ y con una luminosidad que fue incrementándose hasta alcanzar los $2 \cdot 10^{32} \mathrm{~cm}^{-2} \mathrm{~s}^{-1}$ en Octubre. Durante los años 2013 y 2014 el LHC no estuvo en funcionamiento, y se utilizó este tiempo para prepararlo para la nueva etapa que comenzó en el año 2015, en el cual se alcanzó una energía de $\sqrt{s}=13 \mathrm{TeV}$, una energía que nunca antes había sido alcanzada y cercana a la de su diseño original (14 TeV).

\subsection{El detector ATLAS}

ATLAS ( A Torodial LHC AparatuS) es un detector de partículas multipropósito del LHC, diseñado y construido para estudiar las colisiones protón-protón (y de iones pesados) y un gran espectro de procesos físicos en la escala de energía del TeV.

El esquema general del detector se muestra en la Figura 2.2, donde se señalan los componentes principales. ATLAS está diseñado en capas de subdetectores que cumplen diferentes roles en la identificación de las partículas producidas durante las colisiones (ver Figura 2.3). Desde el punto de la colisión hacia afuera ATLAS se compone de un detector interno de trazas (ID) compuesto de un detector de píxeles, un detector de bandas de silicio (SCT) y un detector de radiación de transición (TRT). Por sobre el detector interno se encuentra un solenoide superconductor que genera un campo magnético de $\sim 2 \mathrm{~T}$, a fin de curvar la trayectoria de las partículas cargadas. A continuación están ubicados los calorímetros. En primer lugar el calorímetro electromagnético para medir la energía cinética de electrones y fotones, y posteriormente el calorímetro hadrónico para medir la energía de los jets. En la capa más externa se encuentra el espectrómetro de muones que le da a ATLAS el tamaño total de aproximadamente $45 \mathrm{~m}$ de largo y más de $25 \mathrm{~m}$ de alto. Intercalado con éste se encuentra el sistema de toroides que genera el campo magnético de $\sim 4 \mathrm{~T}$ para curvar la trayectoria de los muones hacia el final de su pasaje por el detector ATLAS.

El detector ATLAS se divide geométricamente en dos regiones, la parte central denominada barrel, y la región de las tapas llamadas end-caps. En cada una de estas regiones la ubicación de los subdetectores es distinta. En la región barrel, los subdetectores están ubicadas como cilindros concéntricos, mientras que en la región end-cap están ubicados como discos consecutivos perpendiculares a la dirección del haz.

\subsubsection{Sistema de coordenadas}

El sistema de coordenadas y la nomenclatura utilizada para describir el detector ATLAS y las trayectorias de las partículas que emergen de las colisiones son resumidas en esta sección, ya que se utilizarán a lo largo de la tesis. Se define como origen de coordenadas al punto de interacción nominal, mientras que la dirección del haz define el eje $z$ y el plano $x-y$ es el transversal a la dirección del haz. El eje $x$ se define desde el punto de interacción, apuntando al centro del anillo del LHC, y el eje $y$ se define apuntando hacia arriba.

Para describir la posición de los distintos subdetectores y la trayectoria de las partículas dentro de ATLAS se utilizan frecuentemente sistemas de coordenadas cilíndricas o polares. El radio $R$ se define como la distancia perpendicular al eje del haz. El ángulo azimutal $\phi$ es medido alrededor del eje del haz, mientras que el ángulo polar $\theta$ es el ángulo respecto al eje del haz.

Una cantidad muy importante utilizada en física de altas energías es la llamada rapidez: 


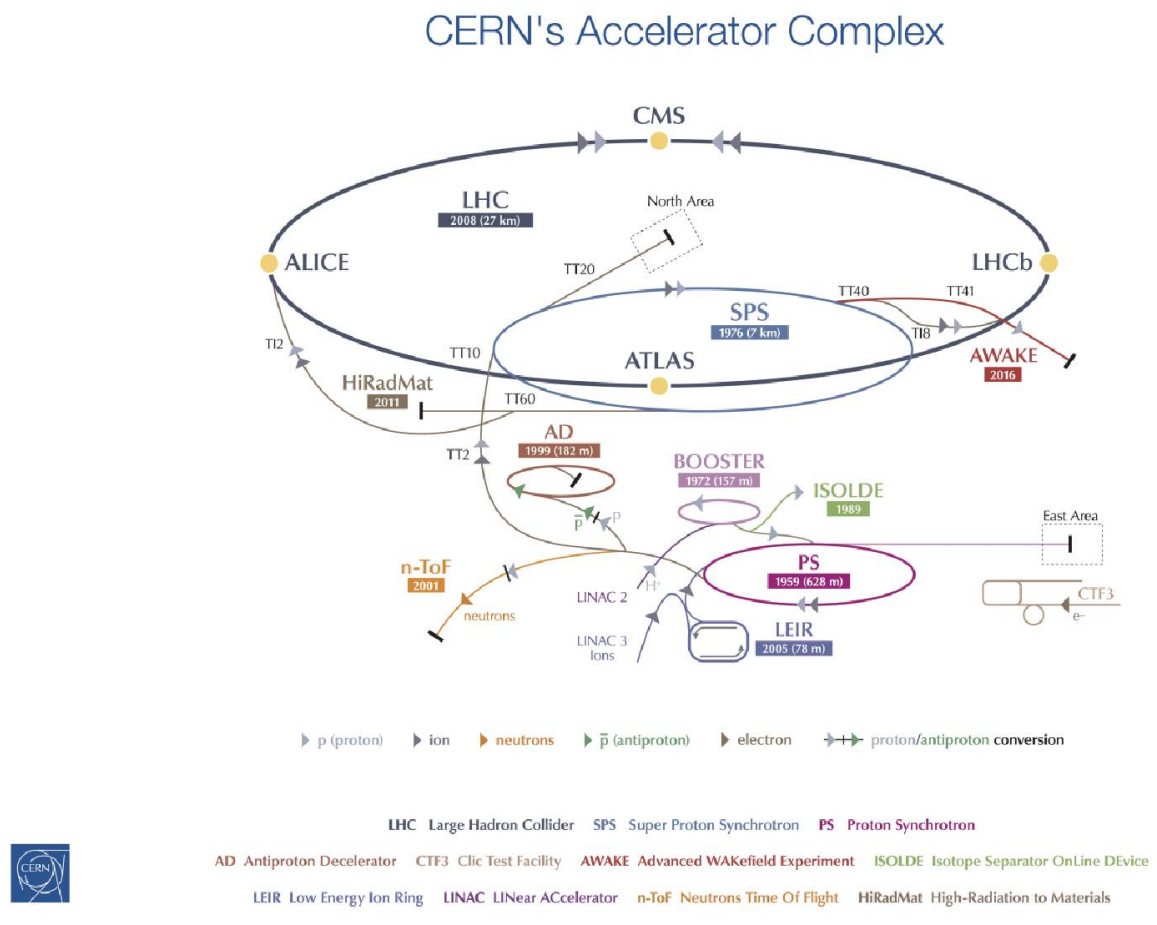

Figura 2.1: Complejo de aceleradores del CERN, incluyendo al LHC y a la serie de aceleradores utilizados para proveer de protones al LHC. También pueden verse los diferentes experimentos ubicados en el acelerador.

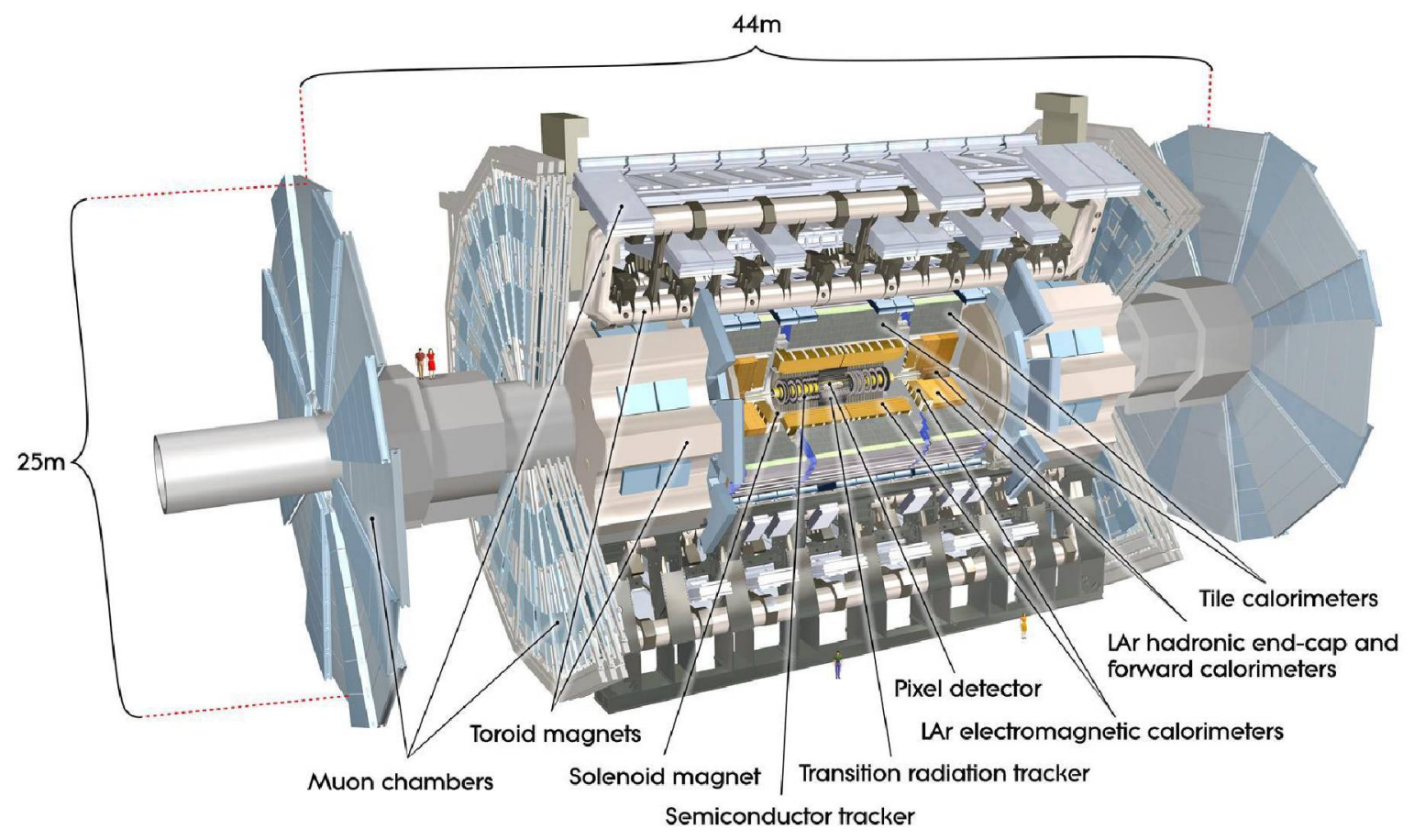

Figura 2.2: Esquema general del detector de ATLAS. Las dimensiones del detector son $25 \mathrm{~m}$ de altura y $44 \mathrm{~m}$ de largo. El peso promedio es de aproximadamente 7000 toneladas. 


$$
y=\frac{1}{2} \ln \left(\frac{E+p_{z}}{E-p_{z}}\right)
$$

donde $E$ es la energía total de la partícula y $p_{z}$ es la componente longitudinal de su impulso. En el límite de altas energías esta cantidad se aproxima (en forma exacta para objetos no masivos) por la llamada pseudorapidez, $\eta$, relacionada con el ángulo polar $\theta$ como:

$$
\eta=-\ln \tan \left(\frac{\theta}{2}\right)
$$

La razón detrás de esta transformación de coordenadas es el hecho que la multiplicidad de partículas producidas es aproximadamente constante como función de $\eta$, y que la diferencia de pseudo-rapidez entre dos partículas es invariante frente a transformaciones (boosts) de Lorentz a lo largo de la dirección del haz. En el caso de colisiones hadrónicas, la fracción del impulso del protón adquirida por cada uno de las partones interactuantes es desconocida. Parte de este impulso es transferido en la interacción dura, mientras cierta fracción remanente escapa el detector a lo largo del haz. Así, no es posible reconstruir el movimiento longitudinal del centro de masa en la interacción, y aplicar leyes de conservación sobre la cinemática de cada evento. Sin embargo, dado que los protones inciden a lo largo de la dirección del haz, el impulso total transverso es conservado durante la colisión. Por esta razón, solo las componentes transversales son utilizadas en la descripción de la cinemática del evento, por ejemplo $p_{\mathrm{T}}(=p \sin \theta)$. En términos de la pseudo-rapidez, se define la energía transversa $\left(E_{\mathrm{T}}=E \sin \theta\right)$ de una partícula como:

$$
E_{\mathrm{T}}=\frac{E}{\cosh \eta}
$$

donde $E$ es su energía total.

\subsection{Los subdetectores de ATLAS}

\subsubsection{El detector interno}

El esquema del detector interno se muestra en la Figura 2.4 y a continuación se describen los distintos componentes del mismo. Este sistema combina detectores de muy alta resolución para distancias cortas al punto de interacción con detectores continuos de trazas a distancias más lejanas. El detector interno está contenido dentro del solenoide que provee un campo magnético nominal de $2 \mathrm{~T}$.

\section{Detector de Píxeles}

Más cerca del punto de interacción se encuentra el detector de píxeles [59], cuyo objetivo principal consiste en la medición de la posición de trazas de partículas cargadas con la más alta precisión posible y es de vital importancia para la reconstrucción de los vértices primarios y secundarios. El principio de detección para partículas cargadas es la medida de la deposición de la carga inducida en una capa de silicio por ionización. Se compone de tres capas en la región barrel (a 4, 10 y $13 \mathrm{~cm}$ del tubo del haz) y tres discos en cada end-cap. La primer capa, conocida como capa- $B$, está ubicada a $50.5 \mathrm{~mm}$ del punto de interacción, permitiendo obtener una resolución óptima del parámetro de impacto. El sistema contiene en total 80 millones de sensores electrónicos, capaces de resolver la posición de las partículas mejor que $14 \mu \mathrm{m}$.

\section{Detector Semiconductor de Trazas (SCT)}

Por fuera del detector de píxeles se encuentra el detector semiconductor de trazas (SCT) que consta de ocho capas de detectores de micro bandas de silicio que provee puntos de alta precisión en las 


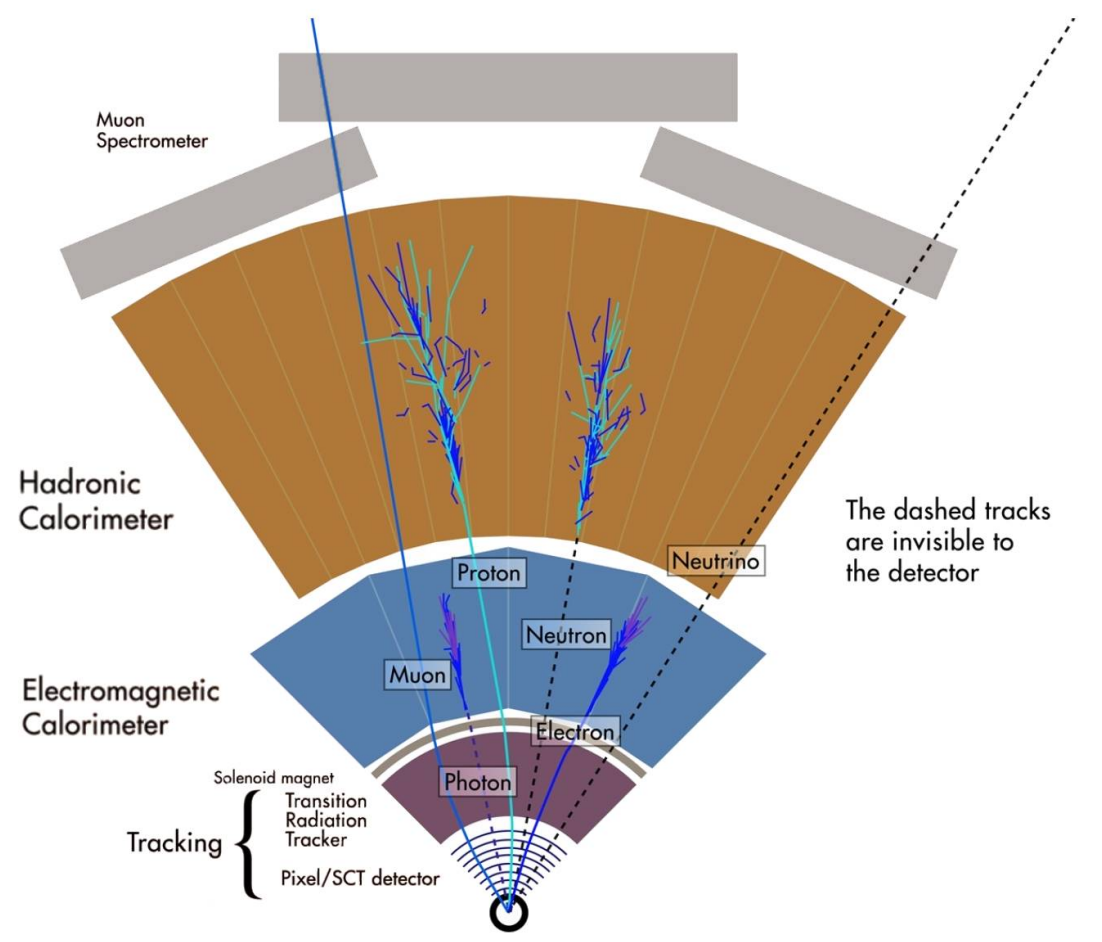

Figura 2.3: Esquema del corte transversal del detector de ATLAS, ilustrando los distintos subdetectores y el pasaje de las distintas partículas.

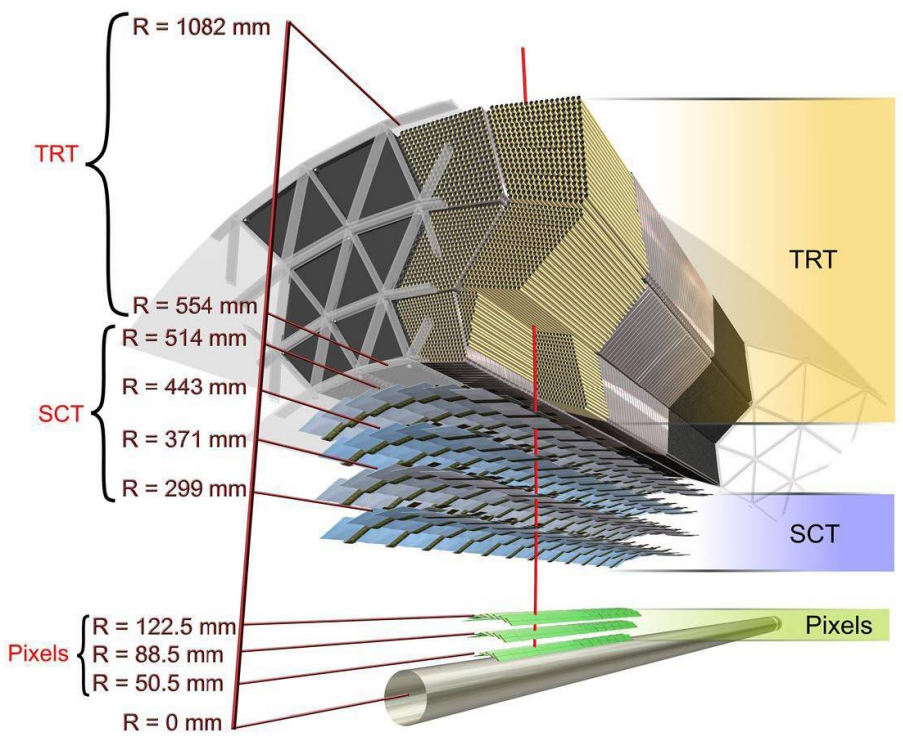

Figura 2.4: Esquema del detector interno mostrando la traza de una partícula cargada de $p_{\mathrm{T}}=10 \mathrm{GeV}$ atravesándolo. La trayectoria atraviesa el tubo del haz de berilio, las tres capas del detector de píxeles de silicio (Pixels), las cuatro capas dobles de sensores semiconductores (SCT), y aproximadamente 36 tubos contenidos en los módulos del detector por radiación de transición (TRT). 
coordenadas $(\mathrm{R} \phi, \mathrm{z})$. La resolución espacial es de $16 \mu \mathrm{m}$ en $\mathrm{R} \phi$ y de $580 \mu \mathrm{m}$ en z y tiene 6.2 millones de canales. Las trazas pueden distinguirse si están separadas más de $\sim 200 \mu \mathrm{m}$. El SCT cubre el rango de pseudorapidez de $|\eta|<2.5$.

\section{Detector de Radiación de Transición (TRT)}

La parte más externa del detector de trazas es el detector de radiación de transición (TRT). Este detector está basado en el uso de tubos detectores que pueden operar a alta frecuencia de eventos gracias a su pequeño diámetro $(4 \mathrm{~mm})$ y el aislamiento de sus hilos centrales en volúmenes de gas individuales.

El TRT además de detectar el pasaje de partículas cargadas, detecta la radiación de transición que permite distinguir entre partículas cargadas pesadas y livianas. La separación entre señales de trazas y de radiación por transición se hace analizando tubo por tubo impactos de alto umbral e impactos de baja señal. El largo de los tubos varía según la zona del detector, llegando hasta los $144 \mathrm{~cm}$ en la zona central. El barrel contiene 50000 tubos y las end-caps contienen 320000 tubos orientados radialmente. El número total de canales es de 420000 y la resolución espacial es de $0.17 \mathrm{~mm}$.

\subsubsection{Calorímetros}

Un esquema de los calorímetros de ATLAS puede verse en la Figura 2.5. Consta de un calorímetro electromagnético cubriendo la región de pseudorapidez $|\eta|<3.2$, un calorímetro hadrónico en la sección barrel cubriendo la región $|\eta|<3.2$, calorímetros hadrónicos en las end-cap cubriendo la región $1.5<|\eta|<3.2$, y calorímetros forward cubriendo $3.1<|\eta|<4.9$.

\section{Calorímetro electromagnético}

El calorímetro electromagnético [60] se divide en una parte central $(|\eta|<1.475)$ y los end-caps $(1.375<|\eta|<3.2)$. La parte central está compuesta por dos mitades, separadas por una distancia pequeña $(6 \mathrm{~mm})$ a $z=0$. Las tapas del calorímetro están divididas en dos ruedas coaxiales, una rueda externa cubriendo la región $1.375<|\eta|<2.5$ y una parte interna que cubre la región $2.5<|\eta|<3.2$.

El calorímetro electromagnético es un detector de muestreo de argón líquido (LAr) con electrodos de kapton en forma de acordeón y planchas absorbentes de plomo. El espesor total del calorímetro electromagnético es $>24 X_{0}$ en el barril y $>26 X_{0}$ en las tapas, donde $X_{0}$ es la longitud de radiación.

En la región dedicada a los estudios de física de precisión $(|\eta|<2.5)$ el calorímetro electromagnético está segmentado en tres secciones longitudinales.

La sección de las bandas (strips) que tiene un espesor constante de $\sim 6 X_{0}$ en función de $\eta$, está equipado con bandas finas de $4 \mathrm{~mm}$ de largo en la dirección $\eta$. Esta sección actúa como un detector de pre-cascada aumentando la capacidad de identificación de partículas, (como por ejemplo la distinción entre $\gamma$ y $\pi_{0}$ o entre electrón y $\pi^{ \pm}$) y dando una precisa medición de la posición en $\eta$. La sección del medio está segmentada transversalmente en torres cuadradas de $\Delta \phi \times \Delta \eta=0.025 \times 0.025\left(4 \times 4 \mathrm{~cm}^{2}\right.$ en $\eta=0$ ). El espesor total del detector hasta el final de la sección del medio es $\sim 24 X_{0}$. La sección más externa tiene una granularidad de $\Delta \phi \times \Delta \eta=0.025 \times 0.05$ y su espesor varía entre 2 y $12 X_{0}$.

\section{Calorímetro hadrónico}

El calorímetro hadrónico de ATLAS [61] cubre el rango $|\eta|<4.9$ usando diferentes materiales. La parte del barrel de este sistema consiste en un calorímetro de muestreo que utiliza acero como absorbente y tejas centelladoras como material activo. Las tejas están ubicadas radialmente y apiladas en profundidad. 
La estructura es periódica en z. Las tejas tienen un espesor de $3 \mathrm{~mm}$ y el espesor de las placas de acero en un período es de $14 \mathrm{~mm}$. El calorímetro de tejas se extiende radialmente desde un radio interno de $2.28 \mathrm{~m}$ hasta un radio externo de $4.25 \mathrm{~m}$.

En la región de end-caps, el calorímetro hadrónico se compone de dos ruedas de $2.3 \mathrm{~m}$ de radio, perpendiculares al tubo del haz, hechas con placas de cobre y tungsteno como material absorbente y argón líquido como material activo. Estos detectores extienden la aceptancia del calorímetro de ATLAS hasta prácticamente cubrir el ángulo sólido del punto de colisión.

\subsubsection{Espectrómetro de muones}

Los muones de alto $p_{\mathrm{T}}$ generados en el punto de interacción tienen un altísimo poder de penetración y son poco interactuantes. Por ello el espectrómetro de muones [62] se encuentra situado en la parte más exterior del detector ATLAS, alrededor del sistema de imanes de toroides, y está diseñado para obtener mediciones de alta precisión de posición e impulso de muones de alto $p_{\mathrm{T}}$. Este es el subdetector más grande y el que le da a ATLAS su tamaño.

La región del barril está compuesta por tres capas concéntricas de cámaras de trigger y de cámaras de precisión posicionadas a $5 \mathrm{~m}, 7.5 \mathrm{~m}$ y $10 \mathrm{~m}$ del tubo del LHC, cubriendo la región $|\eta|<1$. Las regiones de las tapas están compuestas por cuatro capas de cámaras de trigger y cámaras de precisión a $|z|=$ $7.4 \mathrm{~m}, 10.8 \mathrm{~m}, 14 \mathrm{~m}$ y $21.5 \mathrm{~m}$ cubriendo el rango de $1.0<|\eta|<2.7$. Hay una pequeña brecha en $|z|=0$ que permite el acceso de los servicios al ID.

El espectrómetro de muones es el subdetector más grande de ATLAS, construido dentro y alrededor de los imanes toroidales. Los muones son altamente penetrantes y son las únicas partículas (excepto las invisibles que no interactúan) que llegan al sistema de muones. Los muones pierden parte de su energía mientras penetran las capas internas de ATLAS antes de llegar al espectrómetro de muones. La pérdida de energía tiene que ser tenida en cuenta utilizando los depósitos de energía en los calorímetros.

\subsection{El sistema de trigger}

La parte central del sistema de adquisición de datos de ATLAS es el «trigger». El sistema de trigger puede ser pensado como un filtro que selecciona, del gran número de colisiones que tienen lugar en el experimento, los eventos que serán almacenadas para su posterior procesamiento y análisis.

El LHC está diseñado para proveer colisiones $p p$ de $\mathcal{O}(1) \mathrm{GHz}$, considerando una frecuencia de cruce de haces de $40 \mathrm{MHz}$ y $\sim 23$ interacciones por cruce. Dado que la mayoría de los eventos no son de interés para los análisis de física, y también debido a las limitaciones de almacenamiento y de poder de cómputo, el flujo de datos incidente debe ser reducido al máximo permitido para su almacenamiento permanente $(\sim 400 \mathrm{~Hz})$ [3]. Esta reducción se logra mediante una rápida y eficiente preselección de eventos, conocida como trigger.

Esencialmente, el sistema de trigger de ATLAS [2] consiste en una selección de eventos basada en tres niveles: Nivel 1 (L1), Nivel 2 (L2) y Filtro de eventos (EF), donde los dos últimos conforman el High Level Trigger (HLT). Cada nivel permite analizar los eventos con mayor detalle, aumentando la precisión de los criterios de selección y la complejidad de los algoritmos utilizados. El sistema de adquisición de datos transfiere y almacena los datos seleccionados por el trigger.

El L1 se encarga de la selección inicial, reduciendo la frecuencia de eventos que pasan al siguiente nivel a $\sim 75 \mathrm{kHz}$. Debido al tamaño limitado de las memorias temporales donde se guardan los datos de cada subdetector y al considerable tiempo de vuelo de las partículas hasta el espectrómetro de muones, la decisión debe tomarse en una escala de tiempo muy limitada (2.5 $\mu$ s). El L1 está basado en hardware y selecciona objetos de alto $p_{\mathrm{T}}$ construidos a partir de la información de varios subdetectores. 
Los muones son identificados en las cámaras de trigger descriptas en la sección 2.3.3, mientras que la información de los calorímetros, con una resolución reducida, se utiliza para identificar candidatos a electrones, fotones, jets y taus decayendo hadrónicamente. La posición de cada objeto encontrado define una «región de interés» (RoI) en un evento potencialmente interesante, que se extiende como un cono desde el punto de interacción a lo largo del detector.

En el calorímetro, el L1 se basa en las señales analógicas obtenidas en cada torre del trigger, es decir en la suma de celdas en una ventana $\Delta \eta \times \Delta \phi=0.1 \times 0.1$, definida separadamente para el calorímetro electromagnético y hadrónico. La aceptancia geométrica del L1 está ligada al diseño del detector, donde las medidas de precisión en los calorímetros y la cobertura del detector interno están limitadas a la región $|\eta|<2.5$. El trigger de fotones, electrones, muones y taus debe asegurar la cobertura en esta región. En el caso del trigger de jets, las torres del trigger se extienden hasta $|\eta|<3.2$, mientras que para el cálculo de la energía transversa total (faltante) se utiliza todo el sistema calorimétrico (es decir, $|\eta|<4.9$ ). Los resultados de los subsistemas del trigger son procesados en el Central Trigger Processor, en donde se aplica una serie de selecciones definidas como una combinación de criterios individuales, que pueden ser ajustados según la luminosidad y los requerimientos físicos particulares de cada toma de datos. Las distintas configuraciones (items) están disponibles en el L1, donde se programa el tipo de RoI (EM, TAU, JET, etc.) y los umbrales de energía total y de aislamiento requeridos en cada caso. Por ejemplo, el item L1EM14 acepta eventos donde al menos un (dos) cluster(s) en el calorímetro electromagnético posee(n) $E_{\mathrm{T}} \geq 14 \mathrm{GeV}$.

El segundo nivel del trigger (L2) se centra únicamente en las RoIs donde el L1 encontró actividad, combinando información de todos los subdetectores dentro de cada una ( $\sim 2 \%$ de la cobertura total del detector). El L2 consiste de una serie de algoritmos de reconstrucción y selección especializados, diseñados para reducir la frecuencia de eventos hasta aproximadamente $1 \mathrm{kHz}$. Estos algoritmos están implementados en clusters de procesamiento dedicados que analizan cada evento dentro de un tiempo de latencia medio de $\sim 40 \mathrm{~ms}$. El menor flujo de información en este nivel del trigger permite calcular las variables calorimétricas con mayor precisión y hacer uso de la información de las trazas reconstruidas, haciendo posible la distinción entre fotones y electrones, y el rechazo de fondo proveniente en su mayoría de jets. En general, si bien la selección se basa en las mismas variables que la identificación offline descripta en la sección 3.2 (sobre las características de las lluvias electromagnéticas), los valores de corte en cada variable son relajados (o a lo sumo igualados) respecto a la selección offline, para evitar el rechazo prematuro de candidatos que satisfacen los criterios de identificación durante el análisis final. La última etapa de la selección del trigger se lleva a cabo en el EF, que reduce la frecuencia de eventos $a \sim 400 \mathrm{~Hz}$. En este nivel se tiene acceso a toda la información del evento en los distintos subdetectores de ATLAS, con la máxima granularidad e incluyendo detalles sobre la calibración de energía de los calorímetros, la alineación de los subdetectores y el mapa de campo magnético. El tiempo de latencia relativamente largo disponible para tomar la decisión final sobre el evento $(\langle t\rangle \sim 4 \mathrm{~s})$ permite la reconstrucción completa del mismo, y el refinamiento de las variables y criterios de selección al nivel de aquellos implementados en el análisis offine. Los eventos aceptados por el EF son finalmente grabados a disco y distribuidos, accesibles offline para todos los análisis subsecuentes.

Al igual que en el L1, en cada nivel del HLT se configuran ciertos criterios según el tipo y multiplicidad del tipo partícula que se busca en el evento, y el conjunto de cortes de identificación aplicados. La nomenclatura adoptada como convención en el trigger de ATLAS tiene la forma general L_ipX_Y, donde $\mathrm{L}$ es el nivel del trigger (L2,EF), i la multiplicidad, p la partícula de interés (por ejemplo $\mathrm{g}=$ fotón, e $=$ electrón), $\mathrm{X}$ el $p_{\mathrm{T}}$ mínimo requerido e $\mathrm{Y}$ el tipo de identificación aplicada (loose, tight, etc.). Los distintos criterios del L2/EF y su item asociado en el L1 definen en conjunto una de las «cadenas» del trigger, que toman el nombre de la signatura del HLT (es decir, ipX_Y según la convención anterior) y conforman el «menú» final del trigger. 
Para cada item del trigger se puede asignar además un factor de escala o prescale (PS), que define la frecuencia con la que un dado item es evaluado por el trigger (es decir solo en uno de cada PS eventos). Se habla de una cadena de trigger unprescaled si su factor de escala es PS $=1$ en cada nivel, es decir, es evaluada en todos los eventos. La asignación de estos factores se hace incluso dinámicamente durante una toma de datos, para tener en cuenta el descenso de la luminosidad instantánea con el tiempo y mantener la tasa de procesamiento aproximadamente constante.

\subsection{Modelo computacional y distribución de datos}

El modelo computacional de ATLAS está diseñado para permitir a todos los miembros de la colaboración un acceso ágil, directo y distribuido a la gran cantidad de datos recolectados por el detector $(\sim \mathrm{PB} / \mathrm{año}$ ), así como a las diversas simulaciones MC. El modelo se basa en la tecnología GRID, compartiendo el poder de procesamiento y la capacidad de almacenamiento disponibles en distintos centros de cómputo asociados alrededor del mundo.

El software de ATLAS se desarrolla dentro un entorno C++ común llamado ATHENA [63-65], basado en el proyecto GAUDI [66]. Todo el procesamiento de los datos en ATLAS se realiza dentro de este entorno, incluyendo la implementación y configuración del HLT, la simulación de la respuesta del detector, la generación de las muestras MC de los distintos procesos físicos, y la reconstrucción y análisis de los datos. Los eventos aceptados por el trigger deben ser procesados para reducir su tamaño y ser utilizados para los análisis offline. A la salida del HLT, los eventos son almacenados como RDOs (Raw Data Objects). Luego de aplicar los algoritmos de reconstrucción y calibración, las colecciones de los distintos objetos físicos obtenidas (fotones, electrones, etc.) son almacenadas en formato ESD (Event Summary Data) y AOD (Analysis Data Object), una versión reducida del primero ( $\sim 100 \mathrm{kB} /$ evento). A partir de las ESD/AOD, se ha definido un formato de datos significativamente más pequeño (10-15 kB/evento) conocido como D3PD (Derived Physics Data), sobre el que se realiza el análisis final. Las $D 3 P D$ son ntuplas almacenadas en un formato de archivo accesibles vía el entorno de análisis de datos ROOT [67], que contienen un conjunto de variables para diferentes objetos físicos, según las necesidades de cada grupo de análisis dentro de ATLAS. Para el análisis de esta tesis, se utilizaron las D3PD definidas y producidas en forma centralizada por el grupo de SUSY. La misma cadena de reconstrucción y distribución se aplica a las simulaciones Monte Carlo, a fin de conservar un modelo de análisis único y garantizar la consistencia en la comparación de estas con los datos experimentales.

\subsection{Datos de colisiones $p p$ a $\sqrt{s}=8 \mathrm{TeV}$}

Durante la operación del detector ATLAS, cada toma de datos (Run) durante un haz estable provisto por el LHC, es dividida en bloques de luminosidad $(L B)$ de aproximadamente dos minutos, dentro de los cuales la luminosidad instantánea es prácticamente constante, y en el que se espera que las condiciones del haz sean estables. Producto de la complejidad del experimento y de las demandantes condiciones de funcionamiento del LHC, se pueden observar ocasionalmente ciertas ineficiencias en los diversos subdetectores y/o en la cadena de procesamiento de los datos recolectados. Durante cada Run los distintos subcomponentes del detector son monitoreados y cada problema es registrado, incluyendo que componentes están inactivos, o si hay problemas en la infraestructura o en el haz.

Para asegurar la calidad de los datos a ser considerados en los análisis físicos de ATLAS, los grupos responsables de cada subdetector definen un conjunto de criterios de calidad [68], con los cuales se construyen listas, llamadas GRL (Good Runs List), de las Runs y los rangos de LB dentro de ellas 
que son apropiados para cada tipo de análisis. Se producen de forma centralizada para brindar listas oficiales comunes para los distintos grupos dentro de ATLAS y son distribuidas en un formato XML para luego ser utilizadas durante el análisis final. Cada análisis elige que GRL utiliza dependiendo de su tolerancia a las fallas de los subdetectores.

El presente análisis utiliza el conjunto de eventos recolectados de las colisiones pp a una energía de centro de masa $\sqrt{s}=8 \mathrm{TeV}$ con el detector ATLAS durante el año 2012. Estos eventos recolectados corresponden a una luminosidad total integrada de $21.7 \mathrm{fb}^{-1}$. Dado que en el análisis se utilizan fotones, electrones, muones, jets y energía faltante, es imprescindible que todos los subsistemas del detector ATLAS hayan operado en condiciones normales durante la toma de datos. Este requerimiento adicional resulta en una reducción de los datos de $\sim 6 \%$, dejando una luminosidad total de $\int L d t=$ $20.3 \pm 0.6(2.8 \%) \mathrm{fb}^{-1}[69]$ para análisis físicos.

Otro concepto importante de la toma de datos en ATLAS es el pile-up, el cual ocurre cuando las partículas producidas en más de una colisión partón-partón llegan al detector al mismo tiempo, o más generalmente que sus señales se superponen de una forma que no puede ser separadas. Cuando los bunches de protones colisionan, la probabilidad de una interacción es proporcional a la densidad de partículas, o mejor, al flujo de partículas, el cual se expresa con la luminosidad instantánea. El número de colisiones de partículas real que tienen lugar cuando dos bunches se cruzan es una variable aleatoria que sigue una distribución de Poisson. Para bajas luminosidades, en la mayoría de los cruces de haces, no ocurren colisiones, pero para luminosidades instantáneas altas, en la mayoría de los cruces se producen muchas colisiones de partículas al mismo tiempo. Dependiendo del subdetector y el tipo de medición, puede o no ser posible distinguir entre las partículas provenientes de diferentes interacciones simultáneas. Esto es llamado pile-up in-time. En cambio, el pile-up out-of-time incluye los efectos que se originan cuando el tiempo que el detector necesita para volver a su estado de espera es mayor al tiempo entre cruces de bunches. Una medida cuantitativa del pile-up y la actividad del evento, es el valor medio de interacciones inelásticas $p p$ por cruce de bunches, $\langle\mu\rangle$.

Durante el período de toma de datos del 2012, el pico de luminosidad instantánea aumentó desde $2.74 \cdot 10^{30} \mathrm{~cm}^{-2} \mathrm{~s}^{-1}$ hasta $7.61 \cdot 10^{33} \mathrm{~cm}^{-2} \mathrm{~s}^{-1}$, y el número medio de interacciones por cruce de haz varió entre 5.9 y 36.53. La distribución de la luminosidad acumulada durante la toma de datos y del número de interacciones por colisión pude verse en la Figura 2.6. 


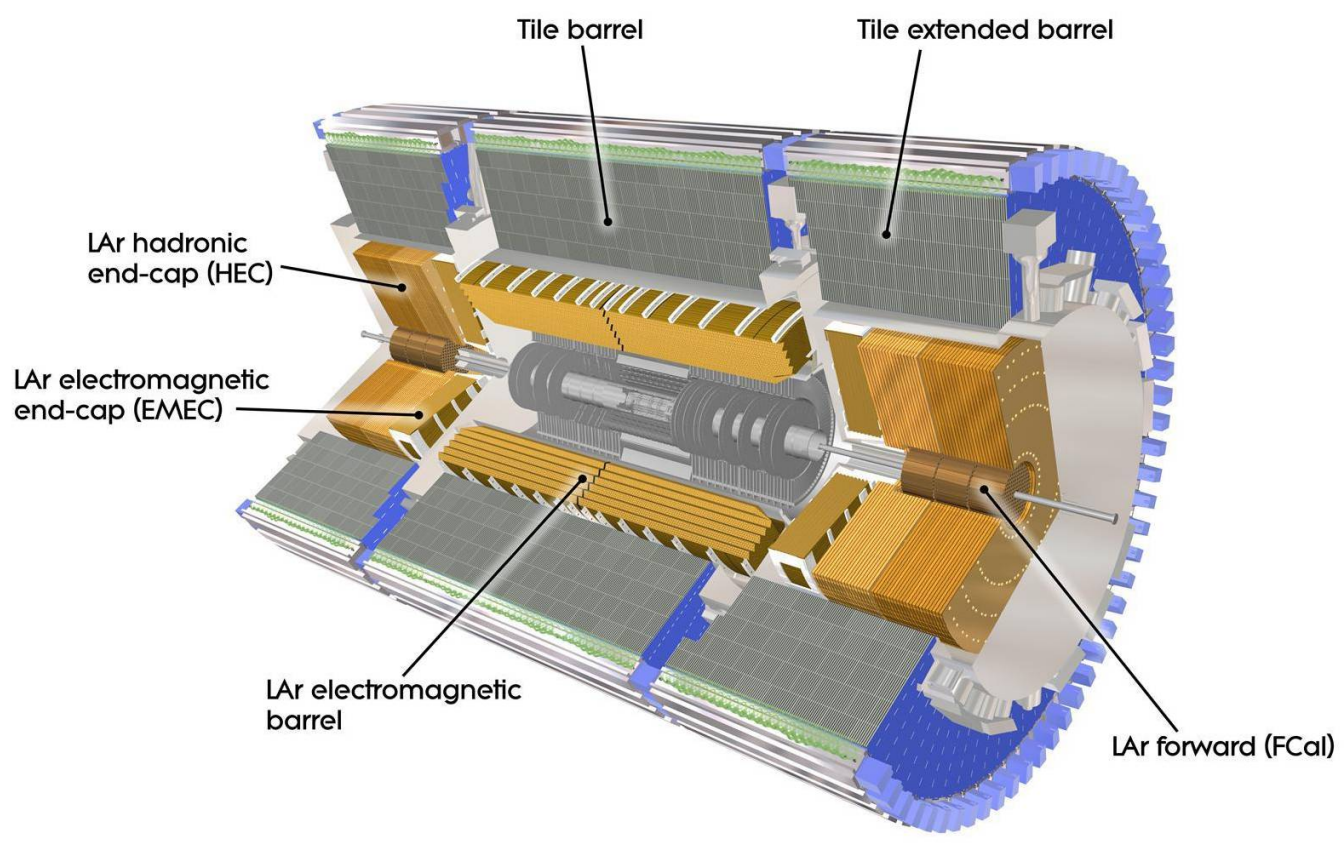

Figura 2.5: Sistema de calorímetros del detector de ATLAS
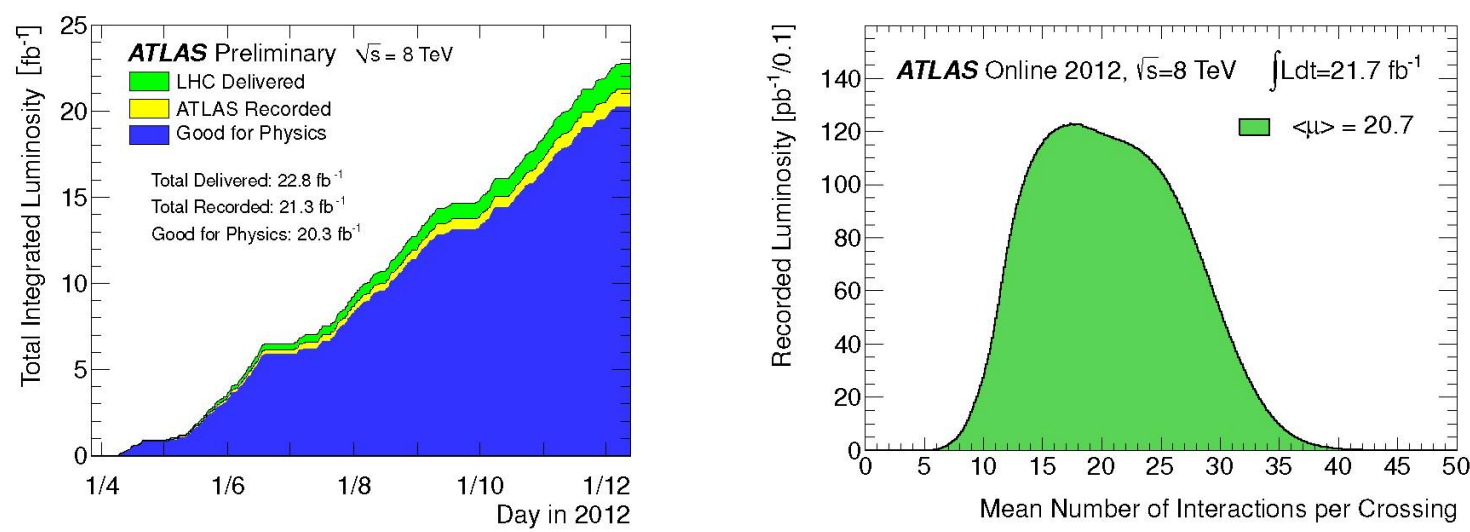

Figura 2.6: Izquierda: Luminosidad acumulada como función del tiempo, entregada por el LHC (verde), guardada por ATLAS (amarillo), y que pasa los criterios de calidad (azul), durante el funcionamiento del LHC con haces estables en colisiones pp a $\sqrt{s}=8 \mathrm{TeV}$ durante el año 2012 [70]. Derecha: Distribución del valor medio del número de interacciones por cruce de haz $(\mu)$ durante la toma de datos en el año 2012 pesado con la luminosidad. La luminosidad integrada y el valor medio de $\mu$ están detallados en la figura. 


\section{RECONSTRUCCIÓN E IDENTIFICACIÓN DE OBJETOS FÍSICOS}

Los objetos físicos resultantes de las partículas originadas en las colisiones $p p$ son reconstruidos a partir de las señales provenientes del detector ATLAS. La reconstrucción de estos objetos no se realiza en tiempo real mientras se recolectan datos (online), sino luego de la toma de datos (offline).

En este capítulo se describe la reconstrucción, calibración e identificación de los principales objetos físicos utilizados en el presente análisis: fotones, jets, muones, electrones y energía faltante. Notar que la correcta identificación de todos los objetos en el evento es requerida para que el cálculo de la energía faltante, que es la energía de las partículas que escapan el detector, sea lo más preciso posible.

\subsection{Reconstrucción de trazas y vértices}

En cada colisión protón-protón del LHC, se produce un gran número de partículas cargadas, resultando en un gran número de impactos en el detector interno de ATLAS. El software encargado de la reconstrucción de trazas debe distinguir los impactos de las diferentes partículas cargadas y determinar las trayectorias que mejor se asocian a las mediciones. El detector interno permite la reconstrucción de las trazas de las partículas cargadas con un momento transverso $p_{\mathrm{T}}>0.5 \mathrm{GeV}$ y $|\eta|<2.5$. Sin embargo, la eficiencia a bajo momento está limitada debido a la gran cantidad de material en el mismo.

La reconstrucción de trazas en el detector interno comienza convirtiendo la información de los subdetectores en puntos espaciales. Seguidamente se realiza la búsqueda de trazas, utilizando dos técnicas. Primero, a partir de la combinación de los puntos en el detector de píxeles y la primera capa del SCT, se forman los candidatos a trazas. Estos candidatos son ajustados y se realiza una limpieza aplicando distintos cortes de calidad. Las trazas seleccionadas son extendidas al TRT, y ajustadas nuevamente utilizando toda la información disponible. De forma complementaria se buscan segmentos de trazas en el TRT que no hayan sido usadas, y se extienden a los demás detectores.

La última etapa consiste en la reconstrucción de los vértices primarios a partir de un algoritmo dedicado, seguido de algoritmos para reconstruir conversiones de fotones y vértices secundarios. Encontrar los puntos de intersección entre las distintas trazas reconstruidas permite identificar el punto de interacción $p p$ como también los vértices de decaimiento de las partículas inestables producidas durante la colisión. La mayoría de las trazas reconstruidas van a originarse en el punto de colisión, indicando el vértice primario de la colisión. Además, algunas partículas pueden decaer a una distancia medible del vértice primario, y las trazas originadas de esos decaimientos pueden utilizarse para identificar los vértices secundarios. Un vértice primario es formado generalmente por la intersección de 20 o más trazas reconstruidas, mientras que los vértices secundarios suelen tener solo 2 o 3 . Primero se 
realiza la búsqueda de los vértices a partir de todas las trazas reconstruidas en el detector interno. La posición de los vértices es determinada mediante un algoritmo de ajuste $\chi^{2}$ sobre los vértices y las trazas de su entorno. Entre todos los candidatos hallados, se elige como primario aquel vértice que $\operatorname{maximiza} \sum_{\text {trazas }} p_{T}^{2}$.

\subsection{Fotones y electrones}

La reconstrucción de fotones y electrones en ATLAS se basa en las deposiciones locales de energía halladas en el calorímetro electromagnético. Como fotones y electrones dejan señales similares en el calorímetro electromagnético, su reconstrucción se realiza en paralelo, distinguiendo entre unos y otros mediante la información de las trazas reconstruidas en el detector interno.

A fin de reducir la gran contaminación de falsos candidatos a fotones en la muestra reconstruida debido, principalmente, al decaimiento de mesones livianos, como por ejemplo $\pi^{0} \rightarrow \gamma \gamma$, se aplica una serie de criterios de identificación, basados en las características de las lluvias electromagnéticas esperadas en cada caso, y también criterios de aislamiento.

\subsubsection{Reconstrucción}

La lógica de reconstrucción se basa en un algoritmo de clusterización que busca deposiciones locales de energía en el calorímetro electromagnético dentro de una ventana rectangular en el plano $(\eta, \phi)$ de tamaño fijo (sliding window algorithm) [71]. La posición de la ventana se ajusta buscando que la energía transversa de todas las celdas contenidas sea un máximo local, con un valor mínimo de $2.5 \mathrm{GeV}$. El tamaño óptimo de la ventana depende del tipo de partícula a reconstruir y de la región del calorímetro. Las lluvias electromagnéticas iniciadas por electrones son en general más anchas que las de fotones, debido a su mayor probabilidad de interacción con el material previo al calorímetro electromagnético y a la radiación de fotones de bremsstrahlung. La energía total y la posición de estos clusters es luego calibrada, separadamente para electrones y fotones, a fin de tener en cuenta diversos efectos como la pérdida de energía en el material inactivo del calorímetro o la deposición lateral y longitudinal fuera del cluster. A partir de simulaciones Monte Carlo, se estima que la eficiencia de esta reconstrucción inicial es del $100 \%$ para objetos con $E_{\mathrm{T}}>20 \mathrm{GeV}$.

Como punto de partida en la separación de electrones y fotones, las trazas, reconstruidas en el detector interno, son asociadas a un cluster si la distancia entre el baricentro del cluster y el punto de intersección de la traza extrapolada con la segunda capa del calorímetro es menor a 0.05 en $\eta$ y 0.05 (0.2) en $\phi$ en la dirección a (opuesto a) la curvatura de las trazas en el campo magnético del solenoide de ATLAS. Solo las trazas asociadas de esta forma con un cluster son retenidas para la reconstrucción de electrones y fotones.

Aquellos clusters electromagnéticos asociados en el espacio $(\eta, \phi)$ con una traza reconstruida con $p_{\mathrm{T}}>0.5 \mathrm{GeV}$ son clasificados como electrones. La definición para fotones es un poco más compleja ya que estos pueden convertir en un par $e^{+} e^{-}$en el volumen anterior al calorímetro. Los fotones convertidos están caracterizados por la presencia de al menos una traza asociada proveniente de un vértice reconstruido en el ID. La probabilidad de conversión varía entre un $40 \%$ y un $80 \%$, aunque solo aquellas que ocurren antes del TRT son eficientemente reconstruidas.

Los fotones son clasificados como no-convertidos si no tienen trazas de un vértice de conversión asociadas al cluster, y como convertidos en el caso contrario. Un vértice de conversión es formado cuando dos trazas que pasan un cierto umbral en el TRT, forman un vértice consistente como provenientes de una partícula no masiva. Para aumentar la eficiencia de reconstrucción de fotones convertidos, los candidatos a conversión donde solo una de las dos trazas es reconstruida (y no tiene ningún impacto 
en la capa más interior del detector de píxeles) también son retenidos.

Un algoritmo final [71] se utiliza para resolver la ambigüedad entre candidatos a fotones convertidos que son también reconstruidos como electrones y permite la recuperación de fotones que fueron inicialmente clasificados como candidatos a electrones. Para candidatos a fotones convertidos que también son reconstruidos como electrones, se evalúa la traza del electrón contra la traza originada del candidato a vértice de conversión asociada al mismo cluster. Si la traza coincide con una traza proveniente de un vértice de conversión, el candidato a fotón convertido es retenido.

Cuando se calcula el $p_{\mathrm{T}}$ del fotón, la energía es tomada siempre del cluster del calorímetro, apropiadamente calibrada [72]. Para fotones no-convertidos, el $\eta$ es calculado usando las dos primeras capas del calorímetro electromagnético. Para fotones convertidos, donde la traza o las trazas que provienen del vértice de la conversión contiene más de tres impactos en el detector de silicio, la dirección $\eta$ se determina extrapolando del cluster del calorímetro al vértice de la conversión. Para fotones convertidos que tienen trazas solo en el TRT, $\eta$ es calculada del calorímetro, como en el caso de los no-convertidos.

De simulaciones Monte Carlo, se calcula que el $96 \%$ de los fotones con $E_{\mathrm{T}}>25 \mathrm{GeV}$ son reconstruidos como candidatos a fotón, mientras que el $4 \%$ restante son incorrectamente reconstruidos como electrones [71].

En el caso de electrones, la eficiencia de reconstrucción es de alrededor de $97 \%$ para electrones con $E_{\mathrm{T}}=15 \mathrm{GeV}$ y alcanza $99 \%$ para $E_{\mathrm{T}}=50 \mathrm{GeV}$. Esta eficiencia varía de $99 \%$ a bajo $|\eta|$ hasta $95 \%$ a alto $|\eta|$ (para electrones con $E_{\mathrm{T}}>15 \mathrm{GeV}$ ) [73].

\subsubsection{Identificación de fotones}

Para distinguir entre candidatos de fotones reales (provenientes de la colisión) de fotones de fondo (provenientes de decaimientos de hadrones), es necesario contar con un algoritmo de identificación con una alta eficiencia y un alto rechazo de fondo para candidatos con $E_{\mathrm{T}}$ desde los $10 \mathrm{GeV}$ hasta la escala del $\mathrm{TeV}$. La identificación de fotones se basa en un conjunto de cortes rectangulares en una serie de variables discriminatorias que caracterizan el desarrollo lateral y longitudinal de la lluvia en el calorímetro electromagnético y la fracción de la lluvia filtrada en el calorímetro hadrónico. Los fotones reales producen generalmente depósitos de energía más angostos en el calorímetro electromagnético y tienen una menor filtración en el calorímetro hadrónico comparado con los fotones de fondo provenientes generalmente de jets, debido a la presencia de hadrones adicionales cercanos al candidato a fotón. Además, los candidatos provenientes de decaimientos aislados $\pi^{0} \rightarrow \gamma \gamma$, están caracterizados por dos máximos locales de energía separados, en las strips de la primer capa del calorímetro, debido a la presencia de los dos fotones cercanos.

A continuación se detallan las variables discriminatorias utilizadas en la identificación de fotones. La primera variable utiliza la energía medida en el calorímetro hadrónico:

- Filtración hadrónica: es la energía transversa depositada en el calorímetro hadrónico, normalizada a la energía transversa del cluster electromagnético

$$
R_{\operatorname{had}_{(1)}}=\frac{E_{\mathrm{T}}^{\text {had }}}{E_{\mathrm{T}}}
$$

En la región de transición barrel-endcap del calorímetro hadrónico $(0.8 \leq|\eta| \leq 1.37)$ se utiliza el depósito de energía en todo el calorímetro hadrónico para minimizar los efectos de la degradación de resolución $\left(R_{\text {had }}\right)$. En el resto del detector, se mide solo la energía depositada en la primera capa del calorímetro hadrónico $\left(R_{\text {had }_{(1)}}\right)$. 
Las siguientes variables utilizan la información de la segunda capa del calorímetro electromagnético:

- Perfil lateral de energía en $\eta$

$$
R_{\eta}=\frac{E_{3 \times 7}^{s 2}}{E_{7 \times 7}^{s 2}}
$$

donde $E_{i \times j}^{s 2}$ es la suma de las celdas en la segunda capa del calorímetro electromagnético contenidas en una ventana $i \times j$ (en unidades de celda $\eta \times \phi)$.

- Perfil lateral de energía en $\phi$

$$
R_{\phi}=\frac{E_{3 \times 3}^{s 2}}{E_{3 \times 7}^{s 2}}
$$

definida en modo similar a $R_{\eta}$. Sin embargo, $R_{\phi}$ se comporta muy distinto para fotones convertidos y fotones no convertidos. Por acción del campo magnético, los electrones y positrones generados en la conversión curvan su trayectoria en direcciones opuestas en $\phi$, dando lluvias electromagnéticas más anchas que los fotones no convertidos en esta dirección.

- RMS del perfil lateral de energía en $\eta$

$$
w_{\eta_{2}}=\sqrt{\frac{\sum E_{i} \eta_{i}^{2}}{\sum E_{i}}-\left(\frac{\sum E_{i} \eta_{i}}{\sum E_{i}}\right)}
$$

mide el ancho lateral de las lluvias electromagnéticas, donde $E_{i}$ es la energía de la i-ésima celda del calorímetro electromagnético contenida en una ventana $3 \times 5$ celdas en $\eta \times \phi$.

Las siguientes son las variables que utilizan la información de la primera capa del calorímetro electromagnético, la cual está compuesta por celdas en forma de bandas (strips) que permiten una muy buena separación entre fotones aislados y fotones provenientes del decaimiento del $\pi^{0}$. La Figura 3.1 muestra el perfil de una lluvia electromagnética típica de cada tipo en eventos de datos reales donde se puede apreciar la estructura del perfil de energía en la primera capa del calorímetro EM en el caso de un decaimiento $\pi^{0} \rightarrow \gamma \gamma$.
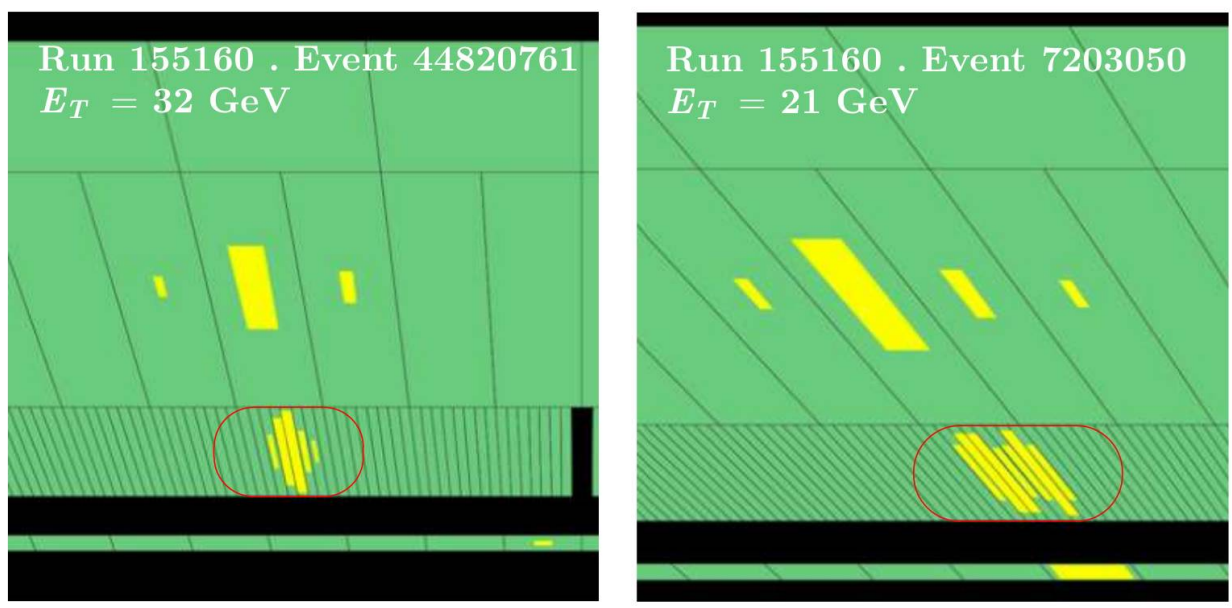

Figura 3.1: Deposiciones de energía típicas para un fotón aislado (izquierda) y para un $\pi^{0}$ decayendo a dos fotones (derecha). 
- Perfil lateral de energía en $\eta$

$$
F_{\text {side }}=\frac{E( \pm 3)-E( \pm 1)}{E( \pm 1)}
$$

mide la contención lateral de la cascada electromagnética a lo largo de $\eta \cdot E( \pm n)$ es la energía en las $\pm n$ celdas alrededor de aquella con la deposición máxima.

- RMS del perfil lateral de energía en $\eta$ (3 strips)

$$
w_{s, 3}=\sqrt{\frac{\sum E_{i}\left(i-i_{\max }\right)^{2}}{\sum E_{i}}}
$$

mide el ancho de la lluvia electromagnética a lo largo de $\eta$ en la primera capa del calorímetro electromagnético usando solo la banda con mayor deposición de energía $\left(E_{i_{\max }}\right)$ y sus vecinas inmediatas.

- RMS del perfil lateral de energía en $\eta$ (total)

$w_{s, \text { tot }}$ está definida de la misma forma que $w_{s, 3}$, pero utiliza todas las bandas de la primera capa del calorímetro electromagnético en una ventana $\Delta \eta \times \Delta \phi=0.0625 \times 0.2$, que corresponde aproximadamente a $20 \times 2$ bandas en $\eta \times \phi$.

- Diferencia al segundo máximo

$$
\Delta E=\left(E_{\max , 2}^{s 1}-E_{\min }^{s 1}\right)
$$

es la diferencia entre la energía de la banda con la segunda energía más grande $\left(E_{\max , 2}^{s 1}\right.$, y la mínima energía $\left(E_{\mathrm{min}}^{s 1}\right.$ ) entre la anterior y la celda con la máxima deposición. En caso de no haber segundo máximo se fija $\Delta E=0$.

- Asimetría de los dos máximos locales en $\eta$

$$
E_{\text {ratio }}=\frac{E_{\max , 1}^{s 1}-E_{\max , 2}^{s 1}}{E_{\max , 1}^{s 1}+E_{\max , 2}^{s 1}}
$$

mide la diferencia relativa entre las energías de las dos celdas con máxima deposición. En caso de no haber segundo máximo se fija $E_{\text {ratio }}=1$.

A partir de estas variables discriminatorias se definen dos conjuntos de cortes: loose y tight. La selección loose se basa solo en las formas de la lluvia en la segunda capa del calorímetro EM y en la energía depositada en el calorímetro hadrónico, y es utilizada especialmente por el trigger. La selección tight agrega además información de la capa de strips del calorímetro, que provee un buen rechazo de fondo, además de ajustar los cortes en las demás variables. Las variables utilizadas en cada conjunto pueden verse detalladas en la Tabla 3.1.

Los cortes de cada conjunto de identificación en las variables que describen la forma de la lluvia son independientes de la energía transversa del candidato a fotón, pero varían según la pseudo-rapidez reconstruida del fotón, para tener en cuenta las variaciones en la cantidad de material y la geometría del calorímetro. La selección tight se optimiza de forma separada para fotones convertidos y no-convertidos para proveer una eficiencia de identificación de alrededor de $85 \%$ para candidatos a fotón con energía transversa $E_{\mathrm{T}}>40 \mathrm{GeV}$ y un alto rechazo de fondo [71]. 
Tabla 3.1: Definición de las diferentes variables usadas para la selección loose (L), medium (M) y tight (T) de fotones y electrones. Las cruces " $x$ " indican las variables que son utilizadas en cada selección. Además de las variables adicionales utilizadas en cada caso, también se incrementan los cortes en las mismas.

\begin{tabular}{|c|c|c|c|c|c|c|c|}
\hline \multirow[b]{2}{*}{ Categoría } & \multirow[b]{2}{*}{ Descripción } & \multirow[b]{2}{*}{ Nombre } & \multicolumn{2}{|c|}{$\gamma$} & \multicolumn{3}{|c|}{$e$} \\
\hline & & & $\mathrm{L}$ & $\mathrm{T}$ & $\mathrm{L}$ & $\mathrm{M}$ & $\mathrm{T}$ \\
\hline Aceptancia & $|\eta|<2.37$, excluyendo $1.37<|\eta|<1.52$ & - & & $\times$ & & $\times$ & $\times$ \\
\hline \multirow[t]{2}{*}{ Fuga hadrónica } & $\begin{array}{l}\text { Cociente entre } E_{\mathrm{T}} \text { en la primera capa del ca- } \\
\text { lorímetro hadrónico y } E_{\mathrm{T}} \text { del cluster electro- } \\
\text { magnético }(|\eta|<0.8 \text { y }|\eta| \geq 1.37)\end{array}$ & $R_{\mathrm{had}_{1}}$ & $x$ & $x$ & $x$ & $x$ & $x$ \\
\hline & $\begin{array}{l}\text { Cociente entre } E_{\mathrm{T}} \text { en todo el calorímetro ha- } \\
\text { drónico y } E_{\mathrm{T}} \text { del cluster electromagnético } \\
(|\eta| \leq 0.8 \text { y }|\eta|<1.37)\end{array}$ & $R_{\mathrm{had}}$ & $\times$ & $x$ & $x$ & $\times$ & $x$ \\
\hline \multirow[t]{3}{*}{$\begin{array}{r}\text { Calorímetro EM } \\
\qquad\left(2^{\text {da }} \text { capa }\right)\end{array}$} & $\begin{array}{l}\text { Cociente entre la suma de las energías de las } \\
3 \times 7 \text { celdas y la suma de } 5 \times 7 \text { celdas, ambas } \\
\text { en torno al centro del cluster }\end{array}$ & $R_{\eta}$ & $x$ & $x$ & $x$ & $x$ & $x$ \\
\hline & Ancho lateral de la lluvia en dirección de $\eta$ & $w_{\eta_{2}}$ & $x$ & $x$ & $x$ & $x$ & $x$ \\
\hline & $\begin{array}{l}\text { Cociente entre la suma de las energías de las } \\
3 \times 3 \text { celdas y la suma de } 3 \times 7 \text { celdas, ambas } \\
\text { en torno al centro del cluster }\end{array}$ & $R_{\phi}$ & & $x$ & $\times$ & $x$ & $x$ \\
\hline \multirow[t]{5}{*}{$\begin{array}{r}\text { Calorímetro EM } \\
\left(1^{\text {ra }} \text { capa }\right)\end{array}$} & $\begin{array}{l}\text { Ancho lateral de la lluvia en } 3 \text { strips alrededor } \\
\text { del máximo }\end{array}$ & $w_{s, 3}$ & & $x$ & & $x$ & $x$ \\
\hline & Ancho lateral total de la lluvia & $w_{s, \text { tot }}$ & & $x$ & & $x$ & $x$ \\
\hline & $\begin{array}{l}\text { Fracción de energía fuera de las } 3 \text { strips cen- } \\
\text { trales pero dentro de las } 7\end{array}$ & $F_{\text {side }}$ & & $x$ & & $x$ & $x$ \\
\hline & $\begin{array}{l}\text { Diferencia entre la energía de la strip con el } \\
\text { segundo mayor depósito y la menor energía } \\
\text { entre los dos primeros máximos locales }\end{array}$ & $\Delta E$ & & $\times$ & & $x$ & $x$ \\
\hline & Asimetría entre el primer y segundo máximo & $E_{\text {ratio }}$ & & $\times$ & & $\times$ & $\times$ \\
\hline \multirow[t]{2}{*}{ Detector Interno } & Impactos en el Pixel $\geq 1$ y en el $\mathrm{SCT} \geq 7$ & - & & & & $\times$ & $x$ \\
\hline & Parámetro de impacto $\leq 1 \mathrm{~mm}$ & - & & & & $x$ & $x$ \\
\hline \multirow[t]{2}{*}{$\begin{array}{l}\text { Calorímetro EM + } \\
\text { Detector Interno }\end{array}$} & $\begin{array}{l}\Delta \eta, \Delta \phi \text { entre la traza extrapolada al caloríme- } \\
\text { tro y el cluster }\end{array}$ & $\Delta \eta, \Delta \phi$ & & & & $\times$ & $x$ \\
\hline & $\begin{array}{l}\text { Cociente entre la energía del cluster y el im- } \\
\text { pulso de la traza }\end{array}$ & $E / p$ & & & & $x$ & $x$ \\
\hline \multirow[t]{2}{*}{$\begin{array}{r}\text { Detector Interno } \\
\text { (TRT) }\end{array}$} & Impactos en el TRT & - & & & & $x$ & $x$ \\
\hline & Fracción de impactos de alto umbral en el TRT & - & & & & $x$ & $x$ \\
\hline
\end{tabular}




\section{Corrección a las variables de identificación}

Dado que la simulación del detector no es perfecta, las distribuciones de las variables discriminatorias utilizadas en la identificación de fotones presentan algunas diferencias que pueden resultar significativas entre datos y MC. Estas diferencias pueden parametrizarse a primer orden como variaciones simples y a partir de estas corregir el MC. Las diferencias para cada variable discriminatoria $\left(\mathrm{DV}^{i}\right)$ se calcula comparando las distribuciones de las mismas en los datos y en una muestra de Monte Carlo con una mezcla similar de eventos de señal y fondo. A partir de esto se calcula la diferencia entre los valores medios, lo que se denomina fudge-factor $\left(\mu^{i}\right)$ [74].

$$
\mu^{i}=\left\langle\mathrm{DV}_{\mathrm{DATA}}^{i}\right\rangle-\left\langle\mathrm{DV}_{\mathrm{MC}}^{i}\right\rangle
$$

Estos factores son utilizados para corregir las variables de las muestras simuladas, y los cortes de identificación son nuevamente aplicados a las variables corregidas.

También es necesario aplicar factores de escala a todos los fotones identificados en las muestras de MC para tener en cuenta las diferencias observadas en la eficiencia de identificación respecto a los datos. Estos factores son derivados de forma separada para fotones convertidos y no-convertidos y son provistos de forma central por el grupo EgAmma de ATLAS.

\section{Aislamiento}

Para la selección final de fotones, se aplica además un corte en la energía transversa de aislamiento $E_{\mathrm{T}}^{\text {iso }}$.

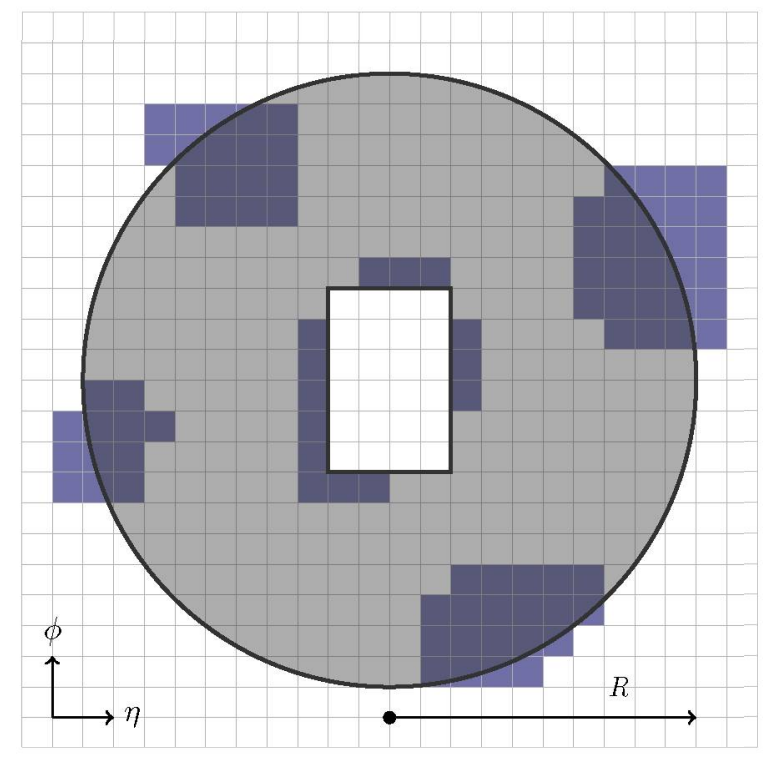

Figura 3.2: Esquema ilustrando el cálculo de la energía de aislamiento del fotón. La grid representa la granularidad de las celdas del calorímetro electromagnético. El cono de color gris de radio $R$ rodea al candidato. La energía del candidato a fotón o electrón está mayormente contenida en el rectángulo central de $5 \times 7$ en $\Delta \eta \times \Delta \phi$.

La energía de aislamiento es una herramienta poderosa para distinguir electrones y fotones directos (producidos en las colisiones) de los falsos candidatos o fotones y electrones no directos provenientes de jets (producidos de decaimientos de hadrones). Esta energía de aislamiento se estima a partir de la energía depositada en un cono alrededor del candidato a electrón o fotón. Para fotones y electrones 
directos, no hay energía depositada en este cono aparte de los objetos de baja energía provenientes de los remanentes de la colisión, interacciones múltiples y ruido de pile-up. El ruido electrónico del calorímetro también contribuye al aumento de la energía de aislamiento. En cambio, para los candidatos falsos y no-directos se observa una energía adicional (potencialmente grande) en dicho cono proveniente de los objetos que los acompañan en el jet.

En la Figura 3.2 se ilustra de forma esquemática como se construye la variable de aislamiento. Se considera un cono alrededor del candidato de un tamaño $R$ (generalmente 0.2 o 0.4 ) y se suma la energía de todas las celdas que pertenecen a un cluster topológico. Los clusters topológicos son construidos usando el cociente señal-fondo de energía y sus vecinos próximos, y son tomados a la escala electromagnética (sin calibrar). La variable final de aislamiento es construida sumando la energía transversa de los clusters con energía positiva cuyo baricentro yace dentro del cono de aislamiento. A esta energía se le resta la energía de las celdas del calorímetro en un rectángulo de $5 \times 7$ celdas (en $\Delta \eta \times \Delta \phi$ en la segunda capa del calorímetro) centrado en el candidato, a fin de remover la energía del propio fotón o electrón.

A pesar que este método es muy eficiente, una fracción de la energía (que aumenta con $p_{\mathrm{T}}$ ) se filtra fuera de este rectángulo y es necesario aplicar una pequeña corrección adicional basada en Monte Carlo.

\subsubsection{Identificación de electrones}

Criterios similares de calidad a los descriptos en la sección anterior se aplican a todos los candidatos a electrones para identificar candidatos falsos debido a problemas instrumentales.

La energía de los electrones es reconstruida a partir de clusters en el calorímetro electromagnético sin tener en cuenta su masa, mientras que la información del detector de trazas es usada para reconstruir su dirección. La escala de energía en MC es corregida para igualar la observada en datos.

Para electrones se definen tres conjuntos de cortes de identificación: loose, medium y tight. Estos se describen con más detalle en [75], y en la Tabla 3.1 pueden verse las variables discriminatorias utilizadas en cada uno.

La eficiencia de identificación fue medida utilizando electrones de los decaimientos de $J / \psi \rightarrow e e$ y $Z \rightarrow$ ee y se combinan para aumentar la precisión de resultados. Esta eficiencia tiene una alta dependencia con $E_{\mathrm{T}}$ y para los criterios más ajustados también con $\eta$, y varía entre $96 \%$ para el criterio loose y $78 \%$ para el criterio tight para electrones con $E_{\mathrm{T}}>15 \mathrm{GeV}$. Al igual que en el caso de los fotones, se aplican factores de escala a los electrones del MC para corregir las diferencias observadas en la eficiencia entre datos y $\mathrm{MC}$.

\subsection{Muones}

Los candidatos a muon son reconstruidos usando el algoritmo de reconstrucción STACO [76] que combina la información del detector de trazas del espectrómetro de muones y el detector interno.

Los muones son clasificados en dos categorías. La primera es denominada combined, y en este caso la reconstrucción de los candidatos a muon comienza en el espectrómetro de muones, donde se reconstruyen los segmentos de sus trazas a partir de los impactos en el detector. Cuando se identifica una traza a partir de estos segmentos, estos se ajustan, permitiendo extraer una medida preliminar del momento. Los segmentos de trazas son luego extrapolados al detector interno donde se busca una traza en el mismo que coincida con aquella del detector de muones. Si se encuentra una traza asociada, estas son combinadas utilizando el algoritmo de combinación estadística (STACO). En ausencia de una traza asociada, la combinación es igualmente aceptada si el $\chi^{2}$ global es menor a un umbral máximo. 
La segunda clasificación comienza buscando trazas en el detector interno que luego son extrapoladas al espectrómetro de muones y asociadas a, al menos, un segmento de traza en el MDT o CSC. Estos muones son llamados segment-tagged.

Para la identificación de muones se implementan tres conjuntos de cortes optimizados para suprimir de forma eficiente trazas falsas y muones que son a veces creados de una alta multiplicidad de impactos en el MS en eventos donde algunas partículas de jets muy energéticos llegan al sistema de muones. Asimismo, discrimina en contra de fondo de muones de decaimientos leptónicos de hadrones pesados. Siguiendo una aproximación similar a la de electrones, se definen los tres conjuntos de cortes: loose, medium y tight. Esencialmente, estos definen el umbral de $p_{\mathrm{T}}$, el requerimiento en el número de impactos, el parámetro de impacto lateral y longitudinal con respecto al vértice primario para rechazar posibles rayos cósmicos, entre otros. Los criterios de identificación detallados pueden verse en [2].

Se requiere además que los candidatos a muon pasen un criterio de aislamiento de la traza: que la suma del $p_{\mathrm{T}}$ de las trazas, excluyendo la traza del muon, en un cono de $\Delta R<0.3$ sea menor que $12 \%$ del $p_{\mathrm{T}}$ del muon. Las trazas tienen que tener cuatro impactos en el detector de silicio y el parámetro de impacto a lo largo de la línea del haz $\left(z_{0}\right)$ debe ser de menos de $10 \mathrm{~mm}$. Es necesario aplicar factores de corrección para que la eficiencia en el MC sea igual a la medida en datos a partir del decaimiento $Z \rightarrow \mu \mu$, los cuales son provistos por el grupo de performance de muones de ATLAS.

\subsection{Jets}

Los partones originados en una colisión $p p$, se observan en el detector como jets de hadrones estables resultado de la hadronización de los mismos. Estos jets atraviesan el detector interno, donde los hadrones cargados dejan su traza, antes de depositar su energía en los calorímetros electromagnético y hadrónico. Dada la complejidad de estos objetos, es necesario contar con una definición de los mismos, es decir, un algoritmo de reconstrucción.

Los jets hadrónicos utilizados en los análisis de ATLAS son reconstruidos utilizando un algoritmo de reconstrucción que empieza con los depósitos de energía de las lluvias electromagnéticas y hadrónicas en los calorímetros. El cuadrimomento es reconstruido a partir de la energía y los ángulos respecto al vértice primario.

Como en el caso de electrones y fotones, los depósitos de energía en los calorímetros son agrupados en clusters. En este caso los clusters son preseleccionados a partir de las celdas calorimétricas que tengan depósitos de energía con una magnitud mayor en al menos $4 \sigma$ del ruido esperado en las celdas. Las contribuciones de energía de todas las celdas vecinas a las celdas preseleccionadas (en 3 dimensiones) son sumadas a la energía de la celda preseleccionada, formando un pre-cluster. A continuación, si la energía depositada en una de las celdas vecinas a la celda preseleccionada es mayor a $2 \sigma$ por encima del valor de fondo de las celdas, estas son agregadas al pre-cluster. Finalmente se agrega una capa de celdas que rodean el pre-cluster formando lo que se llama un cluster topológico, o topo-cluster. Estos topo-clusters son usados por los algoritmos utilizados para encontrar jets.

Existen muchos algoritmos para la reconstrucción de jets, con sus ventajas y desventajas. Los jets utilizados en este análisis son reconstruidos usando el algoritmo anti- $k_{t}$ [77] con un parámetro de distancia $D=0.4$ en el espacio $(\eta, \phi)$ a partir de clusters calorimétricos topológicos [78] formados como se describió previamente.

Este algoritmo hace uso del $p_{\mathrm{T}}$ de cada topo-cluster en el evento y su separación relativa. Para cada par de objetos (los topo-cluster individuales o varios topo-clusters que hayan sido agrupados por el algoritmo) $i$ y $j$ se define la cantidad $d_{i j}$ : 


$$
d_{i j}=\min \left(p_{\mathrm{T}, i}^{-2}, p_{\mathrm{T}, j}^{-2}\right) \frac{\Delta R_{i j}^{2}}{D^{2}}
$$

donde $\Delta R_{i j}^{2}$ es la distancia entre los objetos en el plano $(\eta, \phi)$, y $D$ es el parámetro de distancia que describe el tamaño típico del jet definido por el algoritmo. La iteración es realizada sobre todos los pares constituyentes y también entre cada objeto y el haz, con la cantidad especial $d_{i B}=1 / p_{\mathrm{T}}$. Los objetos $i$ y $j$ son unidos si $d_{i j}$ es menor que $d_{i B}$. Si $\operatorname{mín}\left(d_{i j}, d_{i B}\right)=d_{i B}$, el jet $i$ es declarado un un jet final y removido de la lista de partículas en la nueva iteración. Este proceso es repetido hasta que todos los objetos sean combinados, completando la reconstrucción de todos los jets.

La calibración de la energía de los jets tiene como objetivo corregir la energía del jet medida con los calorímetros a la energía verdadera del correspondiente jet de partículas estables que entra en el detector [79]. Inicialmente, la energía de los jets es reconstruida a partir de las deposiciones en los calorímetros. Esta calibración determina correctamente la energía depositada en el detector solo para lluvias electromagnéticas, por lo que esta escala de energía es denominada escala EM. La escala EM fue establecida utilizando medidas con haces de prueba para electrones en los calorímetros de la zona de barrel [80-82] y end-cap [83,84]. Para lluvias hadrónicas, la escala EM lleva a la subestimación de la energía del jet de un 15-55\%, debido a distintos efectos del detector. Entre otros se encuentran la medición parcial de la energía depositada por los hadrones, las pérdidas de energía en las regiones inactivas del detector, deposiciones de energía de partículas no contenidas en el calorímetro, depósitos de energía de partículas dentro del jet verdadero que no son incluidas en el jet reconstruido. Para compensar la diferencia entre la energía medida de los objetos puramente EM y la energía del jet hadrónico, debe aplicarse una calibración adicional para convertir la escala EM de los calorímetros de ATLAS a la escala hadrónica. En el caso más simple la energía medida del jet es corregida utilizando simulaciones MC y esta calibración es llamada EM + JES.

En este análisis se utiliza una calibración distinta, denominada LCW + JES. Como primer paso los clusters son calibrados usando el método de calibración por pesado de clusters locales (LCW), que consiste básicamente en pesar de forma diferenciada los depósitos de energía que vienen de lluvias en el calorímetro electromagnético o hadrónico. Estas correcciones se aplican a la energía de los topo-clusters independientemente del algoritmo utilizado para la reconstrucción de los jets y son derivadas de simulaciones Monte Carlo. La calibración final en la energía del jet también incluye la escala de energía (JES) que corrige la respuesta del calorímetro con la energía verdadera del jet proveniente de MC.

Como último paso, se requiere que todos los jets reconstruidos pasen ciertos criterios de selección, para remover jets que no provengan de colisiones $[85,86]$.

\subsection{1. $\quad b$-jets}

Para identificar jets provenientes de quarks $b$ ( $b$-jets) se utilizó el algoritmo MV1 [87,88]. Este algoritmo utiliza información sobre los parámetros de impacto de la traza y los vértices secundarios reconstruidos en el detector interno para distinguir jets que contengan hadrones $b$.

\subsection{Energía faltante}

La conservación del momento en el plano transversal al haz $(x-y)$ implica que el momento transverso en el estado final debe ser cero. Cualquier desequilibrio es lo que se llama momento transverso faltante $\left(\boldsymbol{E}_{\mathrm{T}}^{\mathrm{miss}}\right)$ y puede indicar la presencia de partículas indetectables como neutrinos o nuevas partículas estables, o que interactúan débilmente con la materia.

El momento faltante transverso es reconstruido como el valor negativo de la suma del momento 
transverso $\left(p_{\mathbf{T}}\right)$ de todas las partículas detectadas, y su magnitud se denomina con el símbolo $E_{\mathrm{T}}^{\text {miss }}$, y se la llama energía faltante. La medida de $E_{\mathrm{T}}^{\text {miss }}$ depende fundamentalmente del tratamiento y desempeño de los objetos físicos reconstruidos como electrones, fotones, muones, taus, y jets, que son considerados en el cálculo.

La energía faltante transversa es calculada con un algoritmo basado en objetos [89]. El algoritmo utiliza para el cálculo de la energía faltante, los objetos físicos reconstruidos y calibrados descriptos en las secciones anteriores. Los depósitos de energía en el calorímetro (topo-clusters) son asociados a los objetos de alto $p_{\mathrm{T}}$ en el siguiente orden: electrones, fotones, jets y muones. Los depósitos que no están asociados a ningún objeto son incluidos en el término soft. La energía faltante es calculada entonces como la suma de los distintos términos:

$$
E_{x(y)}^{\mathrm{miss}}=\left(E_{x(y)}^{\mathrm{miss}}\right)^{e}+\left(E_{x(y)}^{\mathrm{miss}}\right)^{\gamma}+\left(E_{x(y)}^{\mathrm{miss}}\right)^{\mathrm{jet}}+\left(E_{x(y)}^{\mathrm{miss}}\right)^{\mu}+\left(E_{x(y)}^{\mathrm{miss}}\right)^{\mathrm{soft}}
$$

donde cada término es calculado como el negativo de la suma de los objetos reconstruidos y calibrados más el término soft. El término de electrones $\left(E_{x(y)}^{\text {miss }}\right)^{e}$ incluye electrones que pasan el criterio de identificación medium con $p_{\mathrm{T}}>10 \mathrm{GeV}$. La contribución de fotones $\left(E_{x(y)}^{\mathrm{miss}}\right)^{\gamma}$ utiliza los fotones que pasan el criterio de identificación tight con $p_{\mathrm{T}}>20 \mathrm{GeV}$. En la contribución de jets se incluyen los jets con $p_{\mathrm{T}}>20 \mathrm{GeV}$ en $\left(E_{x(y)}^{\mathrm{miss}}\right)^{\text {jet }}$. La contribución de muones son incluidos en $\left(E_{x(y)}^{\mathrm{miss}}\right)^{\mu}$ utilizando los muones que pasan los criterios de identificación descriptos anteriormente con $p_{\mathrm{T}}>10 \mathrm{GeV}$ exceptuando el criterio de aislamiento. El término restante $\left(E_{x(y)}^{\text {miss }}\right)^{\text {soft }}$ es calculado de los topo-cluster calibrados y las trazas que no son asociadas a ninguno de los objetos reconstruidos, usando un algoritmo de flujo de energía. La falta del término de taus significa que los taus decayendo hadrónicamente se incluyen en el término de jets o el término soft dependiendo del $p_{\mathrm{T}}$ del jet asociado.

Finalmente, la energía faltante $E_{\mathrm{T}}^{\text {miss }}$ y el ángulo azimutal de la misma $\phi^{\text {miss }}$ son calculados como:

$$
\begin{aligned}
& E_{\mathrm{T}}^{\mathrm{miss}}=\sqrt{\left(E_{x}^{\mathrm{miss}}\right)^{2}+\left(E_{y}^{\mathrm{miss}}\right)^{2}} \\
& \phi^{\mathrm{miss}}=\arctan \left(E_{y}^{\mathrm{miss}} / E_{x}^{\mathrm{miss}}\right)
\end{aligned}
$$


PARTE III

ANÁlisis DE DATOS 



\section{MÉTOdOs ESTAdísTiCOS PARA LA BÚSQUEDA DE NUEVA FÍSICA}

Dada la naturaleza probabilística de las colisiones en el LHC y el bajo número de eventos esperado en las búsquedas de nueva física, es necesario contar con una poderosa estructura estadística para interpretar los resultados, especialmente para identificar una nueva señal sobre las posibles fluctuaciones de los fondos del SM.

En este capítulo se introducen los conceptos básicos necesarios para entender el tratamiento estadístico de los datos realizado para esta tesis. Está enfocado en la búsqueda de nueva física en altas energías, su descubrimiento y/o el establecimiento de límites de exclusión.

Este capítulo utiliza como referencia los libros [90,91]. Muchos de los métodos estadísticos más avanzados fueron desarrollados especialmente para el LHC, y se encuentran descriptos en los artículos [92-95]. Además se utilizaron los manuales y artículos que describen las herramientas estadísticas utilizadas $[96,97]$.

\subsection{Funciones de distribución de probabilidad}

Un observable $x$ es por naturaleza frecuentista, es decir, si realizamos el experimento muchas veces, vamos a obtener distintos valores para $x$ y este conjunto de valores dará lugar a una función densidad de probabilidad (pdf) de $x$, que llamamos $f(x)$. En el caso más general, se cuenta con una familia de pdfs $f(x ; \boldsymbol{\theta})$ a la cual se denomina «modelo», y cuyos parámetros representan, por ejemplo, parámetros de la teoría física, alguna propiedad desconocida de la respuesta del detector, o incertezas sistemáticas del análisis.

A continuación se describen algunas funciones de probabilidad importantes que serán utilizadas a lo largo de este capítulo.

\section{Distribución Gaussiana}

La pdf gaussiana (o normal) de una variable continua $x(-\infty<x<\infty)$ se define como:

$$
f(x ; \mu, \sigma)=\frac{1}{\sqrt{2 \pi \sigma^{2}}} \exp \left(\frac{-(x-\mu)^{2}}{2 \sigma^{2}}\right)
$$

Los nombres de los parámetros están claramente motivados por los valores del valor esperado y la varianza de $x$, ya que $E[x]=\mu$ y $V[x]=\sigma^{2}$. Un caso especial es cuando $\mu=0$ y $\sigma=1$, que se denomina gaussiana estándar. La importancia de la distribución normal proviene del Teorema Central del Límite. El teorema dice que la suma de $n$ variables continuas independientes $x_{i}$ con media $\mu_{i}$ y varianza $\sigma_{i}^{2}$ está distribuida de acuerdo a una gaussiana con media $\mu=\sum_{i=1}^{n} \mu_{i}$ y varianza $\sigma^{2}=\sum_{i=1}^{n} \sigma_{i}^{2}$ en el límite 
de $n \rightarrow \infty$. Esto se satisface (bajo ciertas condiciones generales) sin importar las pdfs individuales de $x_{i}$.

\section{Distribución $\chi^{2}$}

La distribución $\chi^{2}$ de una variable continua $z(0 \leq z \leq \infty)$ se define como:

$$
f(z ; n)=\frac{1}{2^{n / 2} \Gamma(n / 2)} z^{n / 2-1} e^{-z / 2}, \quad n=1,2, \ldots
$$

donde $n$ es llamado el número de grados de libertad. Dadas $N$ variables $x_{i}$ gaussianas con media $\mu_{i} \mathrm{y}$ $\sigma_{i}^{2}$, la variable $z=\sum_{i=1}^{N} \frac{\left(x_{i}-\mu_{i}\right)^{2}}{\sigma_{i}^{2}}$ está distribuida de acuerdo a una distribución $\chi^{2}$ con $N$ grados de libertad.

\section{Distribución de Poisson}

La distribución de Poisson es una distribución de probabilidad discreta que describe, a partir de una frecuencia de ocurrencia media, la probabilidad de que ocurra un determinado número de eventos durante cierto período de tiempo. Un ejemplo típico que puede ser descripto por esta distribución es el número de clicks de un contador Geiger en un determinado intervalo de tiempo.

Se la puede pensar como un límite de la distribución binomial cuando el número $(N)$ de experimentos tiende a infinito y la probabilidad $(p)$ a cero, pero el producto es constantes $N p=\nu$. En ese caso la función de probabilidad está dada por:

$$
\operatorname{Pois}(n ; \nu)=\frac{\nu^{n}}{n !} e^{-\nu}
$$

Su valor esperado es $E[n]=\nu$ y la varianza $V[n]=\nu$. Cuando $\nu \rightarrow \infty$ la distribución de Poisson converge a una distribución normal de media $\nu$ y ancho $\sqrt{\nu}$.

\subsection{Estimadores}

La estimación de parámetros de las distribuciones observadas es una de las tareas fundamentales del análisis de datos, y puede considerarse como la medición de un parámetro (que tiene un valor fijo pero desconocido) basado en un número limitado de observaciones experimentales. Dado el experimento, la estimación puntual consiste en determinar un valor único lo más próximo posible al valor verdadero.

Supongamos un conjunto de $N$ observaciones $\boldsymbol{x}=\left(x_{1}, x_{2}, \ldots, x_{N}\right)$, donde las medidas $x_{i}$ son estadísticamente independientes y cada una está descripta por una función de densidad de probabilidad $f(x ; \boldsymbol{\theta})$ que no se conoce, donde $\boldsymbol{\theta}$ es un conjunto de parámetros con valores desconocidos, que tienen valor verdadero $\boldsymbol{\theta}_{0}$.

Un estimador es una función de los datos observados $\boldsymbol{x}$ que provee valores numéricos, los valores estimados $\hat{\boldsymbol{\theta}}$, para el vector de parámetros $\boldsymbol{\theta}$.

Algunas propiedades que es importante que cumplan los estimadores son:

- Consistencia: Un estimador se dice consistente (o asintóticamente consistente) si converge al valor verdadero $\boldsymbol{\theta}_{0}$ con el número de medidas $N$ : $\operatorname{lím}_{N \rightarrow \infty} \hat{\boldsymbol{\theta}}=\boldsymbol{\theta}_{0}$.

- Sesgo: El sesgo está definido como la diferencia entre el valor esperado del estimador y el valor verdadero: $E[\hat{\boldsymbol{\theta}}]-\boldsymbol{\theta}_{0}$ y un estimador es no sesgado cuando el sesgo es cero.

- Eficiencia: Un estimador es eficiente si su varianza $V[\hat{\boldsymbol{\theta}}]$ es lo más pequeña posible. 
Los dos métodos más utilizados para la estimación de parámetros son el de likelihood máximo y el de cuadrados mínimos.

\subsection{Método del likelihood máximo}

Para $N$ mediciones estadísticamente independientes cada una descripta por una pdf $f(x, \boldsymbol{\theta})$, la pdf conjunta para los valores observados $x$ está dada por $f(\boldsymbol{x}, \boldsymbol{\theta})=\prod_{i} f\left(x_{i}, \boldsymbol{\theta}\right)$. La función likelihood se define como la pdf conjunta evaluada en los datos observados,

$$
L(\boldsymbol{\theta})=f\left(\left(x_{1}, x_{2}, \ldots, x_{n}\right) ; \boldsymbol{\theta}\right)=\prod_{i=1}^{N} f\left(x_{i} ; \boldsymbol{\theta}\right)
$$

El estimador de máximo likelihood (MLE) de los parámetros $\boldsymbol{\theta}$ son los valores $\hat{\boldsymbol{\theta}}$ para los cuales la función likelihood $L(\boldsymbol{\theta})$ tiene su máximo global. En la práctica, es conveniente trabajar con el logaritmo de la función likelihood (log-likelihood), y buscar el mínimo del negativo de esta función:

$$
-\ln L(\boldsymbol{\theta})=-\sum_{i=1}^{N} \ln f\left(x_{i} ; \boldsymbol{\theta}\right)
$$

En el límite asintótico, cuando el número de mediciones $N$ tiende a infinito, el MLE es consistente, es decir, para cada parámetro $\theta$ el valor estimado $\hat{\theta}$ converge al valor verdadero $\theta_{0}$. En este límite también el MLE es no sesgado y tiene su menor varianza. Esto significa que ningún otro estimador puede ser más eficiente. Para un número finito de eventos $N$, sin embargo, el MLE tiene un sesgo proporcional a $1 / N$.

Cuando se repite un experimento muchas veces bajo las mismas condiciones, el número de eventos $N$ de un proceso fluctúa de acuerdo a una distribución de Poisson alrededor del valor esperado $\nu$. Este término de Poisson puede incorporarse entonces a la función likelihood, obteniendo lo que se llama «likelihood extendido»:

$$
L(\nu, \boldsymbol{\theta})=\operatorname{Pois}(N ; \nu) \prod_{i=1}^{N} f\left(x_{i} ; \boldsymbol{\theta}\right)
$$

\subsection{Contrastación de hipótesis}

En las secciones anteriores se describió cómo pueden utilizarse los datos observados para estimar parámetros. En esta sección se describirá cómo estos datos experimentales también pueden ser usados para contrastar hipótesis, es decir, para rechazar una teoría o hipótesis, o también para elegir entre hipótesis alternativas.

En general, se llama hipótesis nula $\left(H_{0}\right)$ a la hipótesis sujeta a prueba y usualmente corresponde a la hipótesis que consideramos verdadera. Esta hipótesis es comparada contra una hipótesis alternativa $H_{1}$, o varias $H_{i}$, distintas a $H_{0}$.

En el caso de búsqueda de nueva física en altas energías, la hipótesis que juega el rol de hipótesis nula es la hipótesis de que solo los procesos del SM contribuyen a los datos observados. Esta hipótesis también suele llamarse hipótesis de «solo-fondo». La hipótesis alternativa es la hipótesis en la cual, además de los procesos del SM, también contribuyen procesos de nueva física y se denomina hipótesis de «señal + fondo».

Una hipótesis se dice simple cuando está completamente determinada, mientras que si tiene uno o más parámetros libres, se dice compuesta. Cada hipótesis queda determinada por la pdf que describe a 
los observables bajo esa hipótesis $f(\boldsymbol{x} \mid H)$.

Una vez definidas las hipótesis, se quiere saber si los datos medidos son compatibles con la hipótesis nula o si la hipótesis nula puede ser rechazada en base a estos datos. Con este objetivo se define un «estadístico de prueba» $t(\boldsymbol{x})$ que es función de los datos observados. En el caso de considerar un estadístico de prueba escalar, $t(\boldsymbol{x}) \rightarrow \mathbb{R}$, éste contendrá toda la información de discriminación entre $H_{0}$ y $H_{1}$ en un solo número.

Cada estadístico de prueba tendrá su pdf asociada $g(t \mid H)$ y la decisión sobre la hipótesis estará basada en el valor del estadístico observado $t_{\mathrm{obs}}$ y la definición de una región critica $R$. La región critica queda definida por un valor de corte $t_{c}$ y para el caso de que la hipótesis alternativa tiende a tener valores de $t$ mayores que bajo $H_{0}$, la región critica corresponde a $t \geq t_{c}$ (ver Figura 4.1).

Si el valor observado se encuentra fuera de la región critica (dentro de la región de aceptancia) la hipótesis $H_{0}$ no puede ser rechazada, y si está dentro es rechazada.

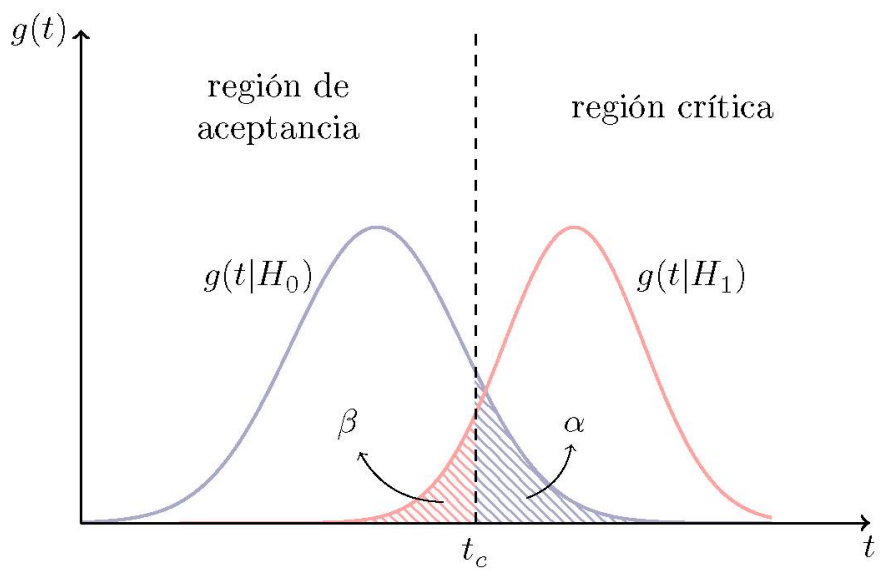

Figura 4.1: Funciones de densidad de probabilidad para el estadístico de prueba $t$ bajo la hipótesis nula $H_{0}$ (azul) y la hipótesis alternativa $H_{1}$ (rojo). Las regiones de aceptancia y rechazo quedan definidas por $t_{c}$.

La probabilidad de rechazar $H_{0}$ siendo ésta verdadera es denominada tamaño de la contrastación $\alpha$ :

$$
\alpha=\int_{R} g\left(t \mid H_{0}\right) d t
$$

lo cual también determina el nivel de significancia como $(100-\alpha) \%$. Este error, de rechazar $H_{0}$ cuando la hipótesis es verdadera, es llamado error de tipo I. Por otro lado el error de aceptar $H_{0}$ cuando es falsa se llama error de tipo II, y su probabilidad de ocurrencia, $\beta$, depende de la hipótesis alternativa $H_{1}$ y del poder del contraste que se define como $(1-\beta)$ :

$$
1-\beta=\int_{R} g\left(t \mid H_{1}\right) d t
$$

En principio, cualquier función de los datos puede ser utilizada como estadístico de prueba, pero un buen estadístico será aquel para el cual sus distribuciones para la hipótesis nula y alternativa estén claramente separadas. Para esto se busca el estadístico de prueba que tenga el mayor poder posible $(1-\beta)$, para un dado tamaño $\alpha$.

Para el caso de hipótesis simples, el teorema de Neyman-Pearson [98] afirma que el estadístico de prueba con mayor poder para un tamaño está dado por: 


$$
t(\boldsymbol{x})=\frac{f\left(\boldsymbol{x} \mid H_{1}\right)}{f\left(\boldsymbol{x} \mid H_{0}\right)}
$$

y la mejor región crítica consiste en $\operatorname{los} \boldsymbol{x}$ que satisfacen $t(\boldsymbol{x})>c_{\alpha}$ donde $c_{\alpha}$ es una constante que se ajusta para que el tamaño sea $\alpha$. Este estadístico de prueba es esencialmente el cociente de los likelihoods para las dos hipótesis.

El nivel de acuerdo entre los datos observados y una hipótesis $H$ es cuantificado calculando el «valor-p», es decir, la probabilidad, bajo la suposición de que $H$ es cierta, de observar datos de igual o menor compatibilidad con la predicción de $H$, respecto a los datos observados.

$$
p_{H}=P\left(t>t_{\mathrm{obs}} \mid H\right)=\int_{t_{\mathrm{obs}}}^{\infty} g(t \mid H) d t
$$

donde $t_{\mathrm{obs}}$ es el valor del estadístico de prueba obtenido con los datos observados.

De esta forma, un valor- $p$ grande denota que la evidencia en contra de $H_{0}$ es débil y un valor- $p$ chico denota que los datos contienen mucha evidencia en contra de $H_{0}$. Se dice que la hipótesis es excluida si el valor- $p$ se encuentra por debajo de un valor específico dado por el tamaño $\alpha$, donde $\alpha \in[0,1]$.

En física de partículas es usual convertir el valor- $p$ en la significancia equivalente, $Z$, definida de forma tal que una variable distribuida normalmente tenga una probabilidad igual a ese valor- $p$ de ser encontrada a más de $Z$ desviaciones estándar a la derecha de su media. Esto es:

$$
Z=\Phi^{-1}(1-p)
$$

donde $\Phi^{-1}$ es el cuantil (la función inversa de la distribución acumulada) de la distribución gaussiana.

También es útil definir, para poder cuantificar la sensibilidad de un experimento, la significancia esperada que se obtendría dados los datos observados bajo la suposición de ciertas hipótesis. Para esto se calcula la significancia de la hipótesis $H_{0}$, respecto a la mediana de la hipótesis $H_{1}$.

\subsection{Descubrimiento}

Generalmente, cuando se modela un fenómeno aleatorio de interés, el modelo elegido para ajustar a las observaciones de dicho fenómeno suele tener varios parámetros, de los cuales solo algunos pueden ser de interés. De manera formal a estos parámetros se los denomina «parámetros de interés» $(\mu)$ y al resto, «parámetros nuisance» $(\boldsymbol{\theta})$, y conviene separarlos explícitamente $\boldsymbol{\theta} \rightarrow(\mu, \boldsymbol{\theta})$.

Para la búsqueda de nueva física es común definir como parámetro de interés a la intensidad de la señal de forma tal que la hipótesis de solo-fondo corresponde a $\mu=0$, y la hipótesis de señal+fondo a $\mu=1$. En general, las incertezas sistemáticas son incluidas en el modelo utilizando parámetros nuisance.

En este escenario, donde hay un único parámetro de interés $\mu, \mathrm{y}$ el resto de parámetros nuisance $\boldsymbol{\theta}$, es conveniente definir el «profile likelihood ratio» (PLR),

$$
\lambda(\mu)=\frac{L(\mu, \hat{\boldsymbol{\theta}})}{L(\hat{\mu}, \hat{\boldsymbol{\theta}})}
$$

donde en el denominador, los valores $\hat{\boldsymbol{\theta}}$ y $\hat{\mu}$ son los valores estimados MLE. En el numerador, los parámetros $\hat{\boldsymbol{\theta}}$ son los valores que maximizan la función likelihood para un valor fijo de $\mu$, es decir que es una función multidimensional que depende solo del parámetro $\mu$. Este proceso de elegir valores específicos de los parámetros nuisance para un valor dado de $\mu$ se lo conoce como profiling. El PLR depende explícitamente de $\mu$ pero es independiente de los parámetros nuisance que han sido "eliminados" vía el profiling. La presencia de los parámetros nuisance que son ajustados a los datos ensanchan la 
función likelihood como función de $\mu$, respecto a la distribución si sus valores estuvieran fijos. De cierta forma reflejan una pérdida de información sobre $\mu$ debido a estos parámetros desconocidos, que suelen ser las incertezas sistemáticas.

En la sección anterior se mostró que el mejor estadístico de prueba para hipótesis simples está dado por el cociente de los likelihoods de las dos hipótesis, de acuerdo al teorema de Neyman-Pearson. Desafortunadamente no hay un equivalente al teorema de Neyman-Pearson para modelos con muchos parámetros libres. Sin embargo, hay una generalización natural basada en el PLR. De la ecuación (4.12) se puede ver que $0 \leq \lambda \leq 1$, y $\lambda$ cercano a 1 implica un buen acuerdo entre datos y el valor hipotético de $\mu$. De forma equivalente conviene utilizar como estadístico de prueba $t_{\mu}=-2 \ln \lambda(\mu)$. En el límite asintótico $(N \rightarrow \infty)$ el teorema de Wilk [99] muestra que $-2 \ln \lambda(\mu)$ sigue una distribución $\chi^{2}$ con un grado de libertad. Esta propiedad sera útil para aproximar la pdf del estadístico de prueba (ver sección 4.7).

Un caso de especial importancia del estadístico $t_{\mu}$ es cuando $\mu=0$. Rechazar la hipótesis de $\mu=0$ es lo que llamamos descubrimiento de una nueva señal. Aunque es cierto que un valor de $\hat{\mu}$ mucho menor a cero representa evidencia contra el modelo de solo-fondo, este tipo de discrepancia no muestran que los datos contengan eventos de señal. Es por este motivo que solo se considera que los datos no tienen un buen acuerdo con la hipótesis de solo-fondo cuando $\hat{\mu}$ es mayor a cero. Para este caso se define $q_{0}$ como,

$$
q_{0}= \begin{cases}-2 \ln \lambda(0) & \hat{\mu}>0 \\ 0 & \hat{\mu} \leq 0\end{cases}
$$

Si los datos fluctúan de tal manera que haya menos eventos que los predichos por el fondo, entonces $\hat{\mu}=0$ y $q_{0}=0$. A medida que el número de eventos crece por encima del número de eventos de fondo esperado (mayor $\hat{\mu}$ ) el valor de $q_{0}$ es mayor, lo que corresponde a un incremento en el nivel de incompatibilidad con la hipótesis de solo-fondo. Para cuantificar el nivel de desacuerdo entre datos y la hipótesis de $\mu=0$ usando el valor observado de $q_{0}$ se calcula el valor- $p$ como,

$$
p_{0}=\int_{q_{0}^{\text {obs }}}^{\infty} f\left(q_{0} \mid \mu=0\right) d q_{0}
$$

En el caso que $p_{0} \leq \epsilon$ donde $\epsilon$ es el tamaño de la contrastación fijado anteriormente, se dice que hay un descubrimiento. En general en física de altas energías suele utilizarse $\epsilon=2.87 \times 10^{-7}$, el cual corresponde a una significancia equivalente $Z=5 \sigma$.

Es importante notar que el hecho de rechazar la hipótesis de solo-fondo en un sentido estadístico es solo parte de descubrir un fenómeno nuevo. Para confirmar la presencia de este nuevo fenómeno es necesario estudiar el acuerdo de este para describir los datos observados.

\subsection{Límites de Exclusión}

Si $p_{0}>\epsilon$ no se puede rechazar $H_{0}$. Esto no significa que todos los valores de $\mu$ bajo $H_{1}$ estén excluidos. En particular, hay valores de $\mu$ a los que el análisis no es sensible y valores que los datos observados no permite excluir.

Para establecer límites superiores en el parámetro $\mu$ se considera $q_{\mu}$,

$$
q_{\mu}= \begin{cases}-2 \ln \lambda(\mu) & \hat{\mu} \leq \mu \\ 0 & \hat{\mu}>\mu\end{cases}
$$

La razón para poner $q_{\mu}=0$ para $\hat{\mu}>\mu$ es que cuando se establece un límite superior, el hecho 
de que $\hat{\mu}>\mu$ representa menos compatibilidad con $\mu$ que los datos obtenidos, y por lo tanto no se considera parte de la región de rechazo de la contrastación.

También es importante notar que $q_{0}$ (utilizado como estadístico de prueba para descubrimiento) no es simplemente un caso especial de la ecuación (4.15), sino que tiene una definición diferente. Es decir, $q_{0}$ es cero si los datos fluctúan hacia abajo $(\hat{\mu}<0)$, pero $q_{\mu}$ es cero si los datos fluctúan hacia arriba $(\hat{\mu}>\mu)$.

Para cuantificar la consistencia de los datos observados con la hipótesis de intensidad de señal $\mu$ se calcula el valor- $p$

$$
p_{\mu}=\int_{q_{\mu}^{\text {obs }}}^{\infty} f\left(q_{\mu} \mid \mu\right) d q_{\mu} \equiv \mathrm{CL}_{s+b}
$$

donde valores chicos de $p_{\mu}$ indican baja compatibilidad con la hipótesis de señal + fondo.

El límite superior con un nivel de confianza del $95 \%$ se obtiene resolviendo la siguiente ecuación:

$$
p_{\mu_{\mathrm{up}}}=0.05
$$

Sin embargo, el límite superior calculado de esta forma tiene un problema: de acuerdo a este, se dice que una señal está excluida a $95 \% \mathrm{CL}, \mathrm{si} \mathrm{CL}_{s+b} \leq 0.05$. Si se considera el caso de $\mu=0$, se espera que por construcción el $\mathrm{CL}_{s+b}$ sea menor o igual que 0.05 con una probabilidad de $5 \%$. Esto significa que el $5 \%$ de los análisis estarían excluyendo modelos con cero señal. Otro problema del $\mathrm{CL}_{s+b}$ es que para dos experimentos con el mismo número chico de eventos de señal esperado pero con un número de eventos de fondo distinto, el experimento con mayor fondo va a imponer mejores límites.

Con motivo de solucionar estos inconvenientes se introduce el método de $\mathrm{CL}_{s}$ [93].

$$
\mathrm{CL}_{s}=\frac{p_{\mu}}{1-p_{b}} \equiv \frac{\mathrm{CL}_{s+b}}{\mathrm{CL}_{b}}
$$

donde $p_{b}$ es el valor del mismo estadístico bajo la hipótesis de solo-fondo,

$$
1-p_{b}=\int_{q_{\mu}^{\text {obs }}}^{\infty} f\left(q_{\mu} \mid 0\right) d q_{\mu} \equiv \mathrm{CL}_{b}
$$

El límite superior $\mathrm{CL}_{s}$ en $\mu, \mu_{\mathrm{up}}$ se obtiene resolviendo $\mathrm{CL}_{s}=0.05$. Se rechazan los valores de $\mu$ si $\mu<\mu_{\text {up }}$ con un nivel de confianza de $95 \%$

Cabe mencionarse para una observación cercana al número de eventos esperado de solo-fondo $\left(\mathrm{CL}_{b} \sim 0.05\right)$ el $\mathrm{CL}_{s}$ da un valor del orden de dos veces el obtenido utilizando el $\mathrm{CL}_{s+b}$.

\subsection{Aproximación asintótica}

Para poder calcular el valor-p de una hipótesis utilizando las ecuaciones (4.14) y (4.16) es necesario conocer las distribuciones muestrales del estadístico de prueba, $f\left(q_{\mu}\right)$.

Como se dijo anteriormente, el teorema de Wilk establece que para el límite de $N \rightarrow \infty$, el estadístico de prueba $t_{\mu}=-2 \ln \lambda$ sigue una distribución $\chi^{2}$ con un grado de libertad, cuando la hipótesis nula es verdadera.

El teorema de Wald [100] generaliza este teorema para el caso de la hipótesis no nula. En este caso el estadístico $-2 \ln \lambda$ sigue una distribución $\chi^{2}$ no central. A partir de este resultado puede mostrarse que para el caso de un solo parámetro de interés vale:

$$
-2 \ln \lambda(\mu)=\frac{(\mu-\hat{\mu})^{2}}{\sigma^{2}}+\mathcal{O}(1 / \sqrt{N})
$$


donde $\hat{\mu}$ sigue una distribución normal con media $\mu^{\prime}$ y desviación estándar $\sigma$. Si $\hat{\mu}$ se encuentra distribuido normalmente y se desprecian los términos $\mathcal{O}(1 / \sqrt{N})$, se puede mostrar que en el régimen asintótico, el estadístico $q_{\mu}$ sigue una distribución $\chi^{2}$ no central con un grado de libertad.

Entonces, en el límite asintótico, el estadístico $q_{\mu}$ puede aproximarse por

$$
q_{\mu}= \begin{cases}\frac{(\mu-\hat{\mu})^{2}}{\sigma^{2}} & \hat{\mu} \leq \mu \\ 0 & \hat{\mu}>\mu\end{cases}
$$

y el valor- $p$ de la hipótesis $H_{\mu}$ es,

$$
p_{\mu}=\int_{q_{\mu}^{\mathrm{bss}}}^{\infty} f\left(q_{\mu} \mid \mu\right) d q_{\mu}=1-\Phi\left(\sqrt{q_{\mu}}\right)
$$

y su correspondiente significancia equivalente,

$$
Z_{\mu}=\Phi^{-1}\left(1-p_{\mu}\right)=\sqrt{q_{\mu}}
$$

Siguiendo los mismos argumentos, el estadístico $q_{0}$ puede aproximarse con,

$$
q_{0}= \begin{cases}\frac{(\hat{\mu})^{2}}{\sigma^{2}} & \hat{\mu} \geq 0 \\ 0 & \hat{\mu}<0\end{cases}
$$

El valor- $p$ para $H_{0}$ entonces es,

$$
p_{0}=\int_{q_{0}^{\text {obs }}}^{\infty} f\left(q_{0} \mid 0\right) d q_{0}=1-\Phi\left(\sqrt{q_{0}}\right)
$$

y la correspondiente significancia,

$$
Z_{0}=\Phi^{-1}\left(1-p_{0}\right)=\sqrt{q_{0}}
$$

Estas aproximaciones permiten conocer las distribuciones muestrales y calcular valores- $p$ y significancias en el caso de un gran número de datos, de una forma simple y computacionalmente poco costosa. A pesar de que estrictamente es válido para $N \rightarrow \infty$, esta aproximación es suficientemente precisa para un número de eventos de fondo $\gtrsim \mathcal{O}(10)$.

Para muestras de datos muy pequeñas, o en casos donde la precisión es importante, siempre pueden validarse estas aproximaciones utilizando la generación Monte Carlo. Para esto es necesario utilizar simulaciones Monte Carlo para generar lo que se denomina «pseudo-experimentos». El procedimiento consiste en generar el conjunto de observables $\boldsymbol{x}$ utilizando la pdf $f(\boldsymbol{x} \mid H)$ y calcular el valor del estadístico de prueba $t(\boldsymbol{x})$ para cada conjunto. Este proceso se repite hasta acumular suficiente estadística en la distribución muestral del estadístico $g(t \mid H)$.

\subsection{Significancia esperada}

En física de partículas la cantidad $s / \sqrt{b}$ ha sido siempre utilizada como una medida de la significancia esperada de descubrimiento [94]. La explicación detrás de esta fórmula es que una cantidad $n$ que sigue una distribución de Poisson con una media grande $s+b$ puede ser aproximada por una variable $x$ distribuida según una gaussiana con media $s+b$ y desviación estándar $\sqrt{s+b}$. En este caso el valor- $p$ de la hipótesis de solo-fondo dada una observación $x$ está dado por,

$$
p=1-\Phi\left(\frac{x-\mu}{\sigma}\right)=1-\Phi\left(\frac{x-b}{\sqrt{b}}\right)
$$


donde $\mu=b$ y $\sigma=\sqrt{b}$ se refieren a la media y la desviación estándar de $x$ suponiendo que $s=0$. Traduciendo este valor- $p$ a significancia,

$$
Z=\frac{x-b}{\sqrt{b}}
$$

La significancia esperada, a diferencia de la observada, se calcula desde la mediana de la hipótesis alternativa. Entonces como la mediana de la hipótesis de señal + fondo, en este caso igual a la media, es $s+b$,

$$
Z_{\exp }=\frac{s}{\sqrt{b}}
$$

que es la ecuación conocida.

De forma general, para el caso de un número de eventos de fondo $b$ conocido con una incerteza despreciable, se puede escribir la función likelihood como,

$$
L(s)=\frac{(s+b)^{n}}{n !} e^{-(s+b)}
$$

y utilizando la aproximación asintótica, la significancia de descubrimiento puede ser aproximada por $Z=\sqrt{q_{0}}$, lo que da como resultado,

$$
Z_{A}=\sqrt{2\left((s+b) \ln \left(1+\frac{s}{b}\right)-s\right)}
$$

Si se desarrolla el logaritmo en la ecuación anterior en $s / b$ se tiene $Z_{A}=s / \sqrt{b}+\mathcal{O}(s / b)$ que es la expresión de la ecuación (4.29) y es válida solo en el límite $s \ll b$.

Si el número de eventos esperado de fondo $b$ no es conocido, uno debe incluirlo como un parámetro nuisance en la función likelihood. Pero como $b$ puede ajustarse para cualquier número de eventos, es necesario introducir información adicional para restringir $b$. En general se suele hacer mediante una medida auxiliar, mirando el número de eventos observados $m$ en una región de control donde se supone ausencia de señal, y considerando que $m$ sigue una distribución de Poisson con media $\tau b$, donde $\tau$ es un factor de extrapolación.

La función likelihood total es el producto de las dos distribuciones de Poisson correspondientes a cada región:

$$
L(s, \boldsymbol{\theta})=\operatorname{Pois}(n ; s+b) \operatorname{Pois}(m ; \tau b)
$$

Utilizando la aproximación $Z=\sqrt{q_{0}}$, válida en el límite de una muestra grande de datos y teniendo en cuenta que los valores esperados son $s+b$ y $\tau b$ para obtener la significancia esperada, se tiene:

$$
Z_{A}=\left[2\left((s+b) \ln \left[\frac{s+(1+\tau) b}{(1+\tau)(s+b)}\right]+\tau b \ln \left[1+\frac{s}{(1+\tau) b}\right]\right)\right]^{1 / 2}
$$

Es útil expresar la ecuación (4.33) en términos de la incerteza que uno quiere atribuirle al fondo basada en la medida de control $m$. El estimador para $b$ está dado por $\hat{b}=m / \tau$, y como la varianza de $m$ es igual a su media $\tau b$, la varianza de $\hat{b}$ es $V[\hat{b}]=\sigma_{b}^{2}=b / \tau$. Usando esto para eliminar $\tau$ de la ecuación (4.33) se obtiene:

$$
Z_{A}=\left[2\left((s+b) \ln \left[\frac{(s+b)\left(b+\sigma_{b}^{2}\right)}{b^{2}+(s+b) \sigma_{b}^{2}}\right]-\frac{b^{2}}{\sigma_{b}^{2}} \ln \left[1+\frac{\sigma_{b}^{2} s}{b\left(b+\sigma_{b}^{2}\right)}\right]\right)\right]^{1 / 2}
$$

y, desarrollando en potencias de $s / b$ y $\sigma_{b}^{2} / b$, se obtiene: 


$$
Z_{A}=\frac{s}{\sqrt{b+\sigma_{b}^{2}}}\left(1+\mathcal{O}(s / b)+\mathcal{O}\left(\sigma_{b}^{2} / b\right)\right)
$$

que es la ecuación conocida, válida cuando $s \ll b$ y $\sigma_{b}^{2} \ll b$. 


\section{ESTRATEGIA GENERAL DEL ANÁLISIS}

En el capítulo 4 se describieron los conceptos básicos de estadística necesarios para realizar un análisis de búsqueda de nueva física. En este capítulo, haciendo uso de estos conceptos, se describe la estrategia general y la construcción del modelo estadístico utilizado en el presente análisis.

\subsection{Estrategia, señal y fondos del SM}

El análisis realizado para esta tesis consiste en la búsqueda de Supersimetría en eventos con un fotón aislado muy energético, jets y gran cantidad de energía faltante. La estrategia general consistió en realizar un experimento de conteo, es decir, considerar como variable estadística al número de eventos observado en una cierta región del espacio de observables, rica en eventos de la señal considerada. Un experimento de conteo puede pensarse en el contexto del likelihood extendido, ecuación (4.6), con $f(x)=1$, el cual se reduce solo al término de Poisson.

El mayor desafío para poder realizar cualquier descubrimiento de nueva física es entender los procesos del SM que pueden dar lugar a un estado final que emule la señal buscada (fondos). En este caso van a ser los que tienen un fotón, jets y energía faltante en el estado final. Estos pueden dividirse en varias categorías. Por un lado, los procesos que dan lugar a eventos con un fotón y energía faltante real, es decir, los que llamamos fondos irreducibles. Estos son:

- $Z(\rightarrow \nu \nu)+\gamma$

- $W(\rightarrow l \nu)+\gamma$

- $t \bar{t}+\gamma$

También es posible que, aunque el proceso no tenga fotones en el estado final, un electrón o un jet sean identificados como un fotón, dando lugar a un estado final idéntico al buscado. En esta categoría están:

- $W(\rightarrow l \nu)+$ jets

- $Z(\rightarrow \nu \nu)+$ jets

- $t \bar{t}$

- $W W, Z Z, W Z$

Y por último, también puede haber procesos que a pesar de no generar energía faltante real, poseen lo que se denomina energía faltante instrumental, proveniente generalmente de la incorrecta reconstrucción de la energía de los jets. De esta manera, pueden dar lugar a eventos con el estado final de interés, los procesos QCD: 
- $\gamma+\mathrm{jets}$

- multijet, con alguno de los jets identificado como fotón

- $Z(\rightarrow l l)+$ jets, donde un leptón o un jet es identificado como un fotón.

Otro componente importante del análisis es la simulación de los procesos de señal. Las muestras de señal pueden depender de uno o muchos parámetros del modelo considerado, como por ejemplo de las masas de las nuevas partículas predichas por Supersimetría. En el caso de considerar un espacio multidimensional del espacio de parámetros del modelo, se utiliza una «grid» de escenarios de señal, en la que cada punto de la grid corresponde a un único punto en el espacio de parámetros. En particular en esta tesis, se consideró un modelo de Supersimetría que puede dar lugar a este tipo de estados finales, para lo cual es necesario contar con la simulación de la señal considerada. En el caso de que no se observe un exceso de eventos en los datos, se pueden establecer límites de exclusión en la grid, excluyendo un subconjunto de los valores de los parámetros considerados.

\subsection{Regiones de señal, control y validación}

Un análisis en el que se quiera estudiar un determinado fenómeno de nueva física requiere la definición de una región en el espacio de observables, donde el modelo de señal predice un exceso significativo de eventos sobre el nivel de fondo predicho en la misma región. A esta región enriquecida en señal se la llama regiôn de señal (SR).

Una de las tareas fundamentales del análisis es entonces, estimar las contribuciones de los procesos del SM que contaminan la región de señal. Para esto existen dos técnicas principales: utilizar directamente simulaciones Monte Carlo, o utilizar métodos basados en los propios datos observados.

La validez de la simulaciones MC está fundamentalmente relacionada a cómo la teoría subyacente modela las observaciones experimentales. Las debilidades de la estimación del fondo a partir de las simulaciones derivan de las debilidades de los modelos teóricos propiamente dichos. En este caso existe una motivación para utilizar métodos que puedan dar una estimación de los fondos a partir de los datos experimentales. Existen distintas formas para estimar un fondo a partir de los datos observados. En el capítulo 8 se describirán los métodos específicos utilizados en este análisis.

En algunos casos se emplea un tercer método para estimar los fondos, que consiste en utilizar la estimación proveniente de las simulaciones MC, pero corregida a partir de los datos. Para esto se define una región de control (CR) en la cual el fondo dominante pueda ser controlado comparándolo con los datos observados en esa misma región. Las CR son diseñadas especialmente para tener una alta pureza en uno de los procesos de fondo y deben estar libres de contaminación de señal.

A través del ajuste a los datos, el número de eventos observado en una CR es usado para normalizar el número de eventos estimado de fondo en todas las regiones, especialmente en la SR. Es decir, las predicciones iniciales de las simulaciones MC son llevadas al nivel observado en la correspondiente CR, usando un factor de normalización calculado en el ajuste. Este factor es utilizado entonces en la extrapolación a las demás regiones.

Otro componente importante del análisis es la validación del método utilizado para predecir los fondos en las SR. Con este objetivo se definen regiones de validación (VR) que se encuentren entre las CR y las SR en términos de los principales observables cinemáticos en los criterios de selección. El diseño de las VR comprende un compromiso entre minimizar la contaminación de la señal, y a su vez ser efectivas en la validación de la extrapolación entre CR y SR. En la Figura 5.1 se puede ver un esquema de las regiones descriptas anteriormente. 


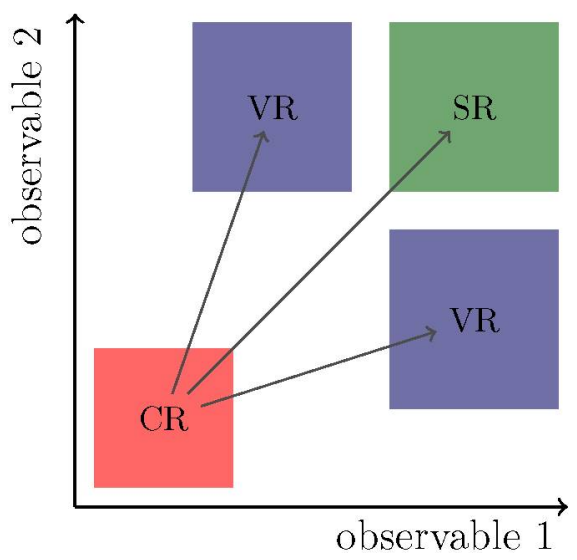

Figura 5.1: Esquema del diseño de las regiones de señal (SR), control (CR) y validación (VR) en términos de dos observables arbitrarios.

Es importante que las CR, VR y SR sean estadísticamente independientes para poder combinar la pdf que modela cada región en una pdf conjunta. Esto es fundamental ya que para estimar los parámetros de la función likelihood se deberá hacer un ajuste simultáneo, que es importante para poder compartir los parámetros de los fondos y las incertezas sistemáticas entre las distintas regiones de forma consistente.

\subsection{Extrapolación y factores de transferencia}

Una de las suposiciones realizada en la sección anterior fue que las variables cinemáticas que se usan para diferenciar CR y SR están bien modeladas después de haber ajustado la pdf total a los datos en las CR. Una vez que los procesos de fondo dominantes son normalizados en las CR, las correspondientes modificaciones en la pdf pueden ser extrapoladas a las VR, las cuales son utilizadas para verificar la validez de esta suposición inicial. Solo cuando se demuestra el acuerdo entre las predicciones del fondo normalizadas y los datos observados en las VR, las predicciones del fondo son extrapoladas a las SR. Se dice que hasta ese momento el analisis se encuentra «blinded», lo cual es fundamental para evitar el uso de predicciones prematuras y un posible sesgo en el resultado final. Es en este paso que los datos observados en las SR son considerados por primera vez en el análisis («unblinding»).

En este procedimiento se utilizan de forma implícita los denominados «factores de transferencia», como se explica a continuación. Las predicciones de los fondo normalizadas en el ajuste son:

$$
\begin{aligned}
& N_{p}^{\text {est }}(\mathrm{CR})=\mu_{p} \times \mathrm{MC}_{p}(\mathrm{CR}) \\
& N_{p}^{\text {est }}(\mathrm{SR})=\mu_{p} \times \mathrm{MC}_{p}(\mathrm{SR})
\end{aligned}
$$

donde $N_{p}^{\text {est }}(\mathrm{CR})$ y $N_{p}^{\text {est }}(\mathrm{SR})$ es el número de eventos estimado para cada proceso de fondo $p$ y $\mathrm{MC}_{p}^{\text {est }}(\mathrm{CR})$ y $\mathrm{MC}_{p}^{\text {est }}(\mathrm{SR})$ es el número de eventos obtenido de las simulaciones $\mathrm{MC}$. El factor $\mu_{p}$ es el factor de normalización obtenido en el ajuste a datos.

Definiendo $N_{p}^{\mathrm{fit}}(\mathrm{SR})$ como el valor ajustado en la CR, se puede escribir de forma equivalente:

$$
\begin{aligned}
N_{p}^{\mathrm{est}}(\mathrm{SR}) & =\mu_{p} \times \mathrm{MC}_{p}(\mathrm{SR}) \\
& \equiv N_{p}^{\mathrm{fit}}(\mathrm{CR}) \times\left[\frac{\mathrm{MC}_{p}(\mathrm{SR})}{\mathrm{MC}_{p}(\mathrm{CR})}\right]
\end{aligned}
$$


El cociente que aparece dentro de los corchete es llamado factor de transferencia (TF). Un aspecto importante de los TF es que las incertezas sistemáticas de los valores estimados de fondo pueden ser parcialmente canceladas en la extrapolación. La incerteza total en el número de eventos de fondo en la SR es por lo tanto una combinación de las incertezas estadísticas en las CR y la incerteza sistemática residual de la extrapolación. Por esta razón las CR suelen definirse con una selección un poco más relajada, para incrementar la estadística, sin aumentar significativamente la incerteza en los TF, y así reducir las incertezas en la SR.

\subsection{Construcción del modelo estadístico}

La función likelihood general para un experimento de conteo como el que se utiliza en el análisis de esta tesis es el producto de los términos de Poisson del número de eventos en la SR y las CR, y una distribución adicional que implementa las restricciones en las incertezas sistemáticas. Puede escribirse como:

$$
\begin{aligned}
L\left(\mu_{s}, \boldsymbol{\mu}_{p}, \boldsymbol{\theta}\right) & =\mathcal{P}_{\mathrm{SR}} \cdot \mathcal{P}_{\mathrm{CR}} \cdot \mathcal{C}_{\mathrm{syst}} \\
& =\operatorname{Pois}\left(n \mid \lambda\left(\mu_{s}, \boldsymbol{\mu}_{p}, \boldsymbol{\theta}\right)\right) \cdot \prod_{i \in \mathrm{CR}} \operatorname{Pois}\left(n_{i} \mid \lambda_{i}\left(\mu_{s}, \mu_{p}, \boldsymbol{\theta}\right)\right) \cdot \mathcal{C}_{\mathrm{syst}}\left(\boldsymbol{\theta}^{0}, \boldsymbol{\theta}\right)
\end{aligned}
$$

Los primeros dos factores $\left(\mathcal{P}_{\mathrm{SR}}\right.$ y $\left.\mathcal{P}_{\mathrm{CR}}\right)$ reflejan las distribuciones de Poisson de $n$, $n_{i}$, el número de eventos observado en cada región. Los valores esperados de las distribuciones de Poisson $\lambda_{i}$ son funciones que dependen de las predicciones de señal y los distintos fondos $p$, los parámetros nuisance que parametrizan las incertezas sistemáticas $\boldsymbol{\theta}$, los factores de normalización para los procesos de fondo $\boldsymbol{\mu}_{p}$ y también la intensidad de la señal $\mu_{s}$. Para $\mu_{s}=0$ se tiene la función likelihood para la hipótesis de solo-fondo y con $\mu_{s}=1$ el valor esperado de señal.

Las incertezas sistemáticas son incluidas usando la pdf $\mathcal{C}_{\text {syst }}\left(\boldsymbol{\theta}^{0}, \boldsymbol{\theta}\right)$, donde $\boldsymbol{\theta}^{0}$ son los valores centrales de las medidas auxiliares alrededor de los cuales $\boldsymbol{\theta}$ puede variarse al maximizar el likelihood. El impacto de los cambios en los parámetros nuisance en los valores esperados están completamente descriptos por las funciones que predicen la cantidad de señal y fondo, $\lambda_{i}$. Para parámetros nuisance independientes $\mathcal{C}_{\text {syst }}$ es simplemente el producto de las pdf correspondientes a las mediciones auxiliares que describen cada una de las incertezas sistemáticas, típicamente una distribución gaussiana $G$ con ancho unidad,

$$
\mathcal{C}_{\text {syst }}\left(\boldsymbol{\theta}^{0}, \boldsymbol{\theta}\right)=\prod_{j \in \mathrm{S}} G\left(\theta_{j}^{0}-\theta_{j}\right)
$$

donde $S$ es el conjunto completo de las incertezas sistemáticas consideradas. Las medidas auxiliares $\theta_{j}^{0}$ son típicamente fijadas a cero, pero pueden variar cuando se generan los pseudo-experimentos.

\subsection{Ajuste del modelo y resultados}

A partir del modelo general descripto en la sección anterior, ecuación (5.4), se pueden realizar distintos tipos de ajuste, dependiendo de las regiones utilizadas y si se incluyen o no las muestras de señal.

Como primer paso se realiza el ajuste únicamente utilizando las regiones de control, y sin incluir las muestras de señal. El propósito de este ajuste es estimar el fondo total esperado en las regiones de validación y las regiones de señal, sin hacer ningún tipo de suposición en algún modelo de señal. Solo se incluyen las muestras de fondo, y las CR se suponen en libres de contaminación de señal. El ajuste 
se realiza utilizando la ecuación (5.4) sin el término de la SR, y las contribuciones de los procesos de fondo dominantes se normalizan al número de eventos observados en esas regiones. A partir de los parámetros del ajuste se puede predecir el número de eventos de fondo en SR y VR. Estas predicciones de fondo son independientes del número de eventos observado en la SR (y VR) ya que solo las CR se usan en el ajuste. Esto permite una comparación no sesgada entre las predicciones y el número de eventos observado en esas regiones. A partir de este ajuste es posible ver si existe un exceso de datos respecto al fondo del SM en las SR, y cuantificar ese exceso calculando el valor- $p$ y la significancia del mismo.

En ausencia de un exceso de eventos significativo, para poder establecer límites en la señal, se incluyen en el modelo las regiones de señal. El ajuste se realiza entonces simultáneamente en las CR y SR, incorporando la muestra de señal en todas las regiones a fin de tener en cuenta la posible contaminación de señal en las CR. Este ajuste se realiza de forma independiente para cada SR, y se repite por cada punto de señal de la grid, para probar el espacio de parámetros del modelo, y establecer límites en las masas de las partículas supersimétricas consideradas. 


\section{MODELO DE SEÑAL Y GENERACIÓN DE EVENTOS MONTE CARLO}

La simulación de los distintos procesos físicos y la respuesta del detector a los mismos es necesaria para poder optimizar y estimar el desempeño de los diferentes análisis. Además, permite que las estrategias utilizadas en la identificación de partículas puedan ser desarrolladas con anterioridad a la toma de datos y las eficiencias de los algoritmos pueden ser puestos a prueba. La preparación de las búsquedas de nueva física necesitan una simulación detallada del detector para estimar su potencial de descubrimiento y para desarrollar métodos óptimos para medir las propiedades de las partículas. Es fundamental un correcto entendimiento de los procesos de señal y de fondo para poder distinguir entre ambos. Una vez que los datos de colisiones reales están disponibles, los datos simulados también resultan necesarios para poder encontrar desviaciones del SM.

La estructura de los eventos de colisiones de altas energías son realmente complejos y no predecibles de primeros principios. Los generadores de eventos permiten separar el problema en varios pasos más simples, algunos de los cuales pueden ser descriptos por primeros principios, y otros necesitan ser basados en modelos apropiados con parámetros ajustados a los datos. Un aspecto central de los generadores es que proveen una descripción del estado final para poder construir cualquier observable y compararlos con los datos de colisiones reales.

En este capítulo se describe como fueron simulados los procesos del modelo de SUSY (sección 6.2) y los procesos del SM (sección 6.3) que dan lugar al estado final de interés. Todas las muestras fueron simuladas utilizando generadores Monte Carlo a $\sqrt{s}=8 \mathrm{TeV}$, a continuación se simuló el pasaje de las partículas generadas por el detector ATLAS, para finalmente ser reconstruidas con los mismos algoritmos que los datos de colisiones reales.

\subsection{Generación de eventos Monte Carlo}

La conexión entre la teoría/fenomenología de SUSY (o cualquier otra teoría de nueva física), y los datos observados en el detector de un colisionador se realiza por medio de un «generador de eventos Monte Carlo» $[101,102]$. Dada una teoría de nueva física, que en general predice la existencia de nuevas partículas y/o interacciones, el generador de eventos permite calcular cómo esa teoría se manifestará en el experimento.

El procedimiento, una vez que se selecciona un modelo particular de SUSY, requiere de varios pasos que se encuentran esquematizados en la Figura 6.1 [103]. En primer lugar es necesario calcular el espectro de masas de las partículas supersimétricas, y sus acoplamientos. A partir de ellos se calculan los anchos y tazas de decaimiento de todas estas partículas, y por último se utiliza el generador de eventos, que toma como entrada las masas y decaimientos calculados previamente, y genera un conjunto 
de eventos a la energía de centro de masa del colisionador. A continuación, para cada evento, se simula el pasaje de las partículas por el detector.

Para las distintas etapas suelen utilizarse herramientas diferentes, por lo que es fundamental contar con un forma estandarizada de intercambio de información entre ellas. Con este objetivo se creó un tipo de archivo llamado SLHA (SUSY Les Houches Accord) [104] que permite organizar toda la información de un cierto modelo de supersimetría (parámetros, masas, decaimientos) en un formato único, de modo que sea fácil de compartir entre los físicos y también entre las distintas herramientas utilizadas.

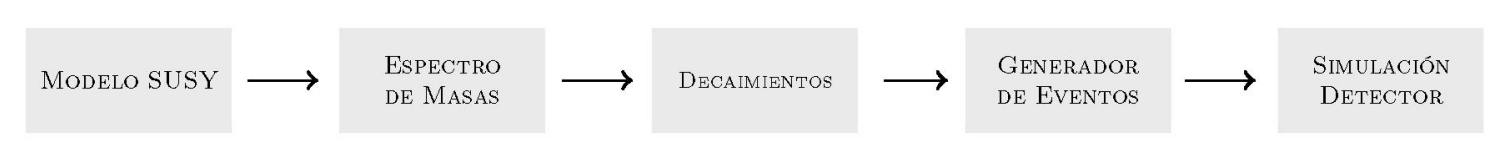

Figura 6.1: Etapas de la simulación de eventos de un modelo particular de SUSY.

\subsubsection{Espectro de masas y decaimientos}

Los modelos de SUSY que se estudian en el LHC suelen ser la teoría efectiva a bajas energías que resulta de una teoría más general, como teoría de supercuerdas, o que involucre un mecanismo particular para el rompimiento de SUSY. Para especificar una teoría efectiva es necesario definir: la simetría de gauge, los campos que la componen y el lagrangiano. Como se describió en el sección 1.2, los efectos del rompimiento de SUSY están codificados en los términos de rompimiento soft del lagrangiano. También es necesario especificar la escala de energía a la cual la teoría efectiva y el lagrangiano son válidos. Como los experimentos prueban física a la escala del $\mathrm{TeV}$, mientras que los parámetros del lagrangiano son frecuentemente especificados a energías mucho más altas $\left(M_{\mathrm{GUT}} \circ M_{P}\right)$, deben usarse las ecuaciones del grupo de renormalización (RGE) para conectar las dos escalas del modelo. Una vez que los parámetros del lagrangiano son conocidos a la escala electrodébil, pueden identificarse las masas físicas de las partículas, diagonalizando las matrices de masa correspondientes, y luego, para tener la precisión suficiente, aplicar las correcciones a ordenes mayores (en general a 1 loop). A partir del modelo también es posible calcular los anchos y tasas de decaimiento de todas las partículas supersimétricas.

Existe una gran variedad de programas que permiten realizar estos cálculos. En este trabajo se utilizó el programa Susyhit [105] que combina Suspect [106] para calcular el espectro de masas, junto con SDECAY [107] y HDECAY [108] para calcular los BRs y anchos de decaimiento. Algunos de estos modos de decaimientos son calculados a NLO en QCD.

Una vez que el espectro de masas y los decaimientos están calculados, pueden usarse como entrada en los generadores de eventos.

\subsubsection{Generador de eventos}

Los programas descriptos anteriormente permiten pasar de un modelo específico a las predicciones en la producción de partículas y sus anchos de decaimientos en estados finales de quarks, leptones, fotones y gluones (y LSP en modelos donde se conserva paridad-R).

El paso siguiente consiste en la generación de los eventos Monte Carlo. Para un dado tipo de colisionador y una dada energía de centro de masa, el generador MC se encarga de generar los eventos esperados para el modelo considerado.

La colisión de dos protones a altas energías en el LHC implica el estudio de las interacciones de los constituyentes de los protones, es decir, de los quarks y gluones. La interacción total de los dos 
protones es complicada, como se muestra en la Figura 6.2 y se puede descomponer en distintos pasos. En todos los componentes del diagrama excepto en la interacción fuerte, dominan los procesos de QCD.

Los protones acelerados por el LHC interactúan para producir las partículas predichas por la teoría. Este proceso de interacción fuerte involucra los partones de los hadrones intervinientes en el proceso. El cálculo se realiza, en general, a primer orden (LO) en teoría de perturbaciones aunque algunos programas pueden incluir algunos procesos a NLO. Esta etapa involucra la convolución con las funciones de distribución partónicas para obtener las secciones eficaces de producción.

Las funciones de distribución partónica se utilizan para describir la subestructura del protón y son usadas por todos los generadores de eventos. ATLAS utiliza la librería LHAPDF $[109,110]$ que contiene un repositorio con una gran cantidad de PDF. Por defecto, y a menos que se indique lo contrario las PDFs CTEQ [111] son las utilizadas en ATLAS.

A partir de la colisión inicial y la producción de las partículas finales (en el caso de SUSY será un par de partículas supersimétricas) se modela el decaimiento de las mismas (en general en varios pasos) hasta el estado final partónico, de acuerdo a los BR predichos por el modelo.

La multiplicidad de partones en el estado final depende, además de los partones producidos en la interacción fuerte, de la radiación en el evento. Pueden producirse partones adicionales por radiación de un gluón o quark ya sea antes de la interacción fuerte (llamada Initial State Radiation, ISR) o después de la interacción fuerte, en la fase de fragmentación (llamada Final State Radiation, FSR). Cada uno de los partones en el estado final comienzan a perder energía, a través de la radiación de gluones. Estos gluones fragmentan en gluones adicionales y pares quark-antiquark. Esto continúa hasta el punto en donde la energía es lo suficientemente baja para recombinar todas las partículas de color en mesones y bariones, proceso denominado «hadronización». Los hadrones decaen subsecuentemente en otros hadrones, leptones y neutrinos. Cada partón proveniente de la interacción fuerte (e ISR/FSR) resulta en una lluvia de partículas, denominada colectivamente «jet».

Finalmente, los remanentes de los haces iniciales tienen que ser modelados para obtener una descripción válida de la física incluyendo no solo la interacción dura sino los procesos soft, lo que suele llamarse evento subyacente (UE).

Todos estos procesos se repiten hasta generar un gran número de eventos, donde cada evento consiste básicamente en los vectores de todas las partículas en el estado final.

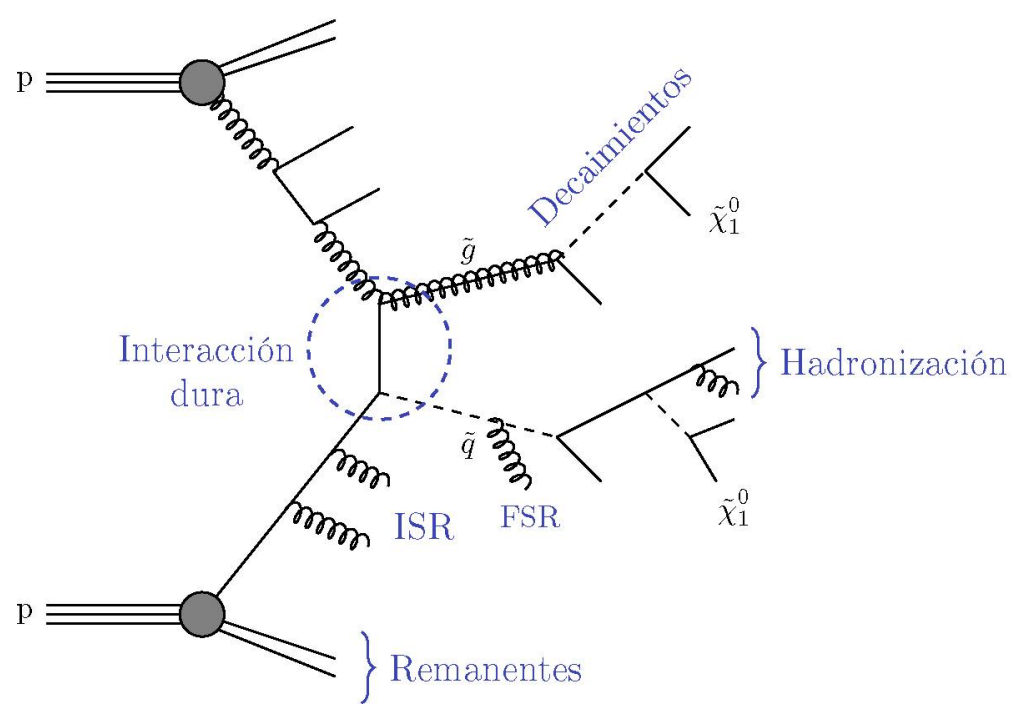

Figura 6.2: Esquema de las distintos pasos implementados en los generadores de eventos Monte Carlo. Adaptado de [103]. 


\subsubsection{Simulación del detector ATLAS}

Para algunos estudios aproximados es posible utilizar directamente las partículas producidas por los generadores Monte Carlo. Pero en el caso general es necesario simular el pasaje de estas partículas por el detector.

A fin de poder estudiar la respuesta del detector para un gran número de procesos físicos y escenarios, se ha implementado una simulación detallada que lleva los eventos de la generación a un formato que es idéntico a las señales en el detector verdadero [112]. La simulación está integrada al software de ATLAS (ATHENA), y utiliza el paquete de simulación GEANT4 [113].

La simulación está dividida así en tres etapas: generación del evento y los decaimientos inmediatos, simulación del detector, y digitalización de los depósitos de energía en las regiones sensibles del detector en los voltajes y corrientes que se encuentran a la salida del detector. Luego, la salida de estos procesos de simulación sirve como entrada a los algoritmos de trigger y reconstrucción de ATLAS, que son idénticos a los que se utilizan en los datos reales.

Existe también una simulación rápida del detector que es utilizada en los casos donde no es necesaria un simulación tan minuciosa de las lluvias electromagnéticas en los calorímetros. Casi $80 \%$ del tiempo de simulación se debe a la simulación de partículas atravesando el calorímetro, y cerca del $75 \%$ se emplea en la simulación de las partículas electromagnéticas. En la simulación rápida ATLFAST [114], se remueven estas partículas electromagnéticas de baja energía y se las reemplaza con lluvias electromagnéticas pre-simuladas. De esta forma se reduce el consumo de CPU notablemente considerando las características de la física en cuestión.

En las simulaciones Monte Carlo, el pile-up in-time es incorporado superponiendo las señales de los procesos físicos de interés con señales correspondientes a un dado número de colisiones adicionales independientes de minimum-bias. Cada evento en una muestra Monte Carlo es producido bajo ciertas condiciones particulares del detector, y bajo un valor particular de pile-up. Existen algunas diferencias en el perfil del pile-up de los datos reales y las muestras MC, en particular debido a que las muestras MC son simuladas previo a la toma de datos con el pile-up previsto que no es exactamente igual al real. Para solucionar esto, se aplica un repesado evento a evento a las muestras MC de manera de modelar las condiciones reales de la muestra de datos bajo estudio.

\subsection{Simulación de la señal de SUSY}

Como se ha mencionado en el sección 1.2, debido al gran número de parámetros libres en los modelos de SUSY, las búsquedas de supersimetría en ATLAS están impulsadas por la fenomenología de los estados finales. El análisis realizado para esta tesis está motivado por estados finales con fotones energéticos, provenientes del decaimiento de un neutralino NLSP en el contexto de modelos GGM.

En los modelos GGM el decaimiento de los estados supersimétricos producidos en las colisiones del LHC va a proceder por medio de decaimientos en cascada hasta el neutralino NLSP que decaerá a un gravitino y una partícula del SM, que dependerá de la naturaleza de la NSLP. Distintas posibilidades para la naturaleza de la NLSP pueden ser consideradas, las cuales dan lugar a estados finales distintos y complementarios entre sí, para cubrir las distintas regiones del espacio de fase de los modelos GGM. Dentro de ATLAS se exploraron cuatro estados finales diferentes: dos fotones, un fotón y un leptón, un fotón y b-jets, y un fotón y jets, todos con energía faltante, correspondiente a los gravitinos LSP. En la Figura 6.3 pueden verse los diagramas de los distintos estados finales posibles dependiendo de la composición del neutralino NLSP.

En particular en esta tesis se focalizó en el análisis de un estado final que consiste en un único fotón, jets y energía faltante. La selección de eventos que se describe en el capítulo 7 fue diseñada para 

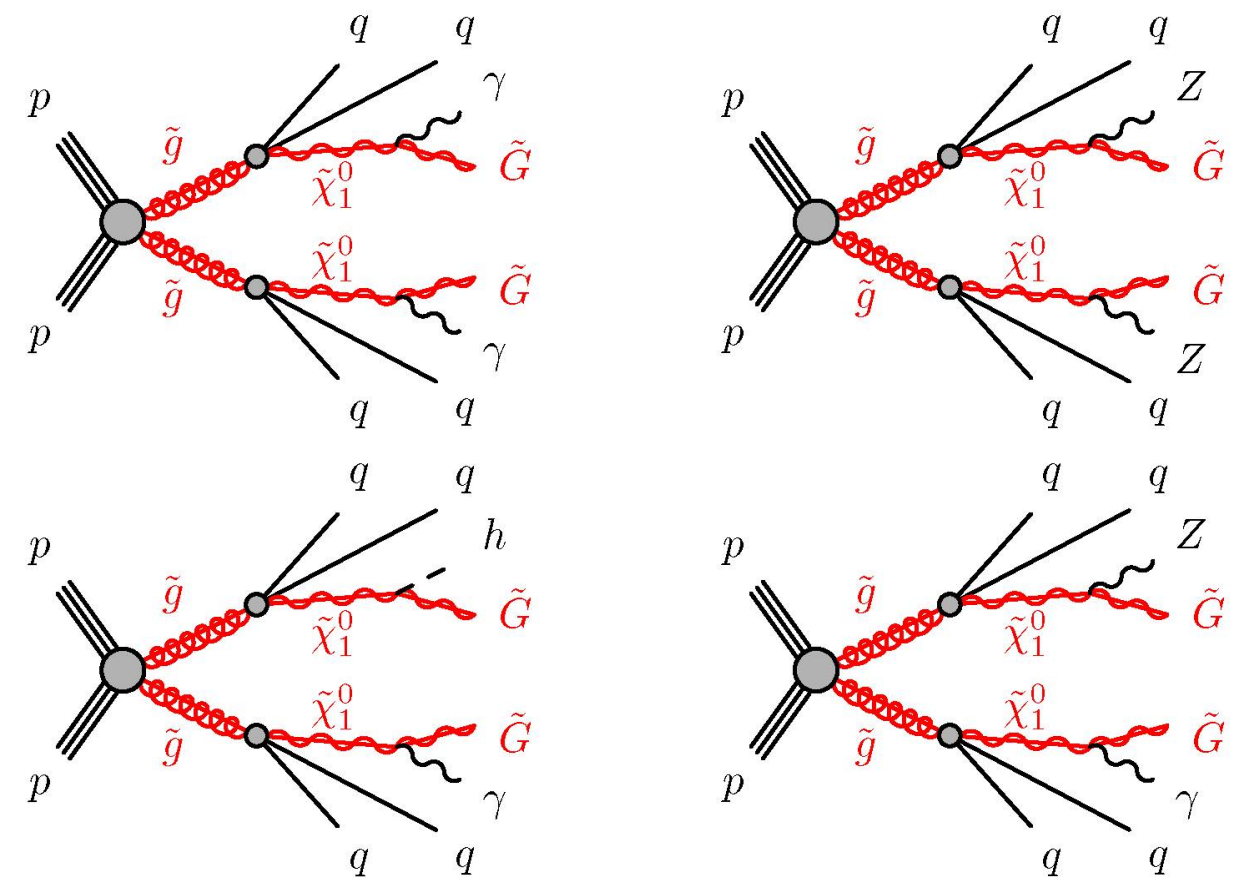

Figura 6.3: Diagramas de Feynman de producción de gluinos, ilustrando los distintos estados finales dependiendo la composición del neutralino NLSP.

maximizar la sensibilidad a pequeñas señales con esta topología general. Cualquier imposición de cortes muy dependientes del modelo fueron evitadas, tratando de mantener el análisis lo más independiente del modelo como fuera posible. Sin embargo, una interpretación en el marco de un modelo específico es inevitable. Con tal motivo se simuló un conjunto de puntos de señal con distintos valores de los parámetros para cubrir la región del espacio de parámetros donde pueda manifestarse dicha señal.

Se utilizó un espectro simplificado en el cual, básicamente, el espacio de parámetros consiste en la escala de producción (por simplicidad, la masa del gluino) y la masa de la NLSP. Todos los demás estados fueron desacoplados ya que no juegan un rol importante en la producción o en el estado final de interés. Esta aproximación es similar a los denominados modelos simplificados utilizados en otras búsquedas. La masa del gluino es el único parámetro libre de las partículas de color para poder determinar un límite conservativo en la masa del mismo. Todos los parámetros de masa soft de los squarks se fijaron en $2.5 \mathrm{TeV}$.

Para maximizar la probabilidad de tener un único fotón en el estado final, es necesario que $\operatorname{BR}\left(\widetilde{\chi}_{1}^{0} \rightarrow \gamma \widetilde{G}\right) \sim 50 \%$. Esto resulta cuando el neutralino más liviano es una mezcla bino-higgsino. Además, el valor de $\mu$ debe ser positivo para suprimir el decaimiento a Higgs, que llevará a un estado final ya cubierto por otro análisis de ATLAS. Para lograr la BR deseada se variaron los parámetros de masa de bino $\left(M_{1}\right)$ y higgsinos $(\mu)$ para las diferentes masas del $\tilde{\chi}_{1}^{0}$, de tal forma que los BR del $\tilde{\chi}_{1}^{0}$ sean aproximadamente constantes:

$$
\begin{aligned}
& \operatorname{BR}\left(\widetilde{\chi}_{1}^{0} \rightarrow \gamma \widetilde{G}\right) \approx 50 \% \\
& \operatorname{BR}\left(\widetilde{\chi}_{1}^{0} \rightarrow Z \widetilde{G}\right) \approx 49 \% \\
& \operatorname{BR}\left(\widetilde{\chi}_{1}^{0} \rightarrow h \widetilde{G}\right) \approx 1 \%
\end{aligned}
$$

Los demás parámetros del modelo se fijan en $M_{2}=2.5 \mathrm{TeV}, \tan \beta=1.5$ y $c \tau_{\mathrm{NLSP}}<0.1 \mathrm{~mm}$. Este último asegura que el neutralino decaiga rápidamente dentro del detector y se logra haciendo al 
gravitino lo suficientemente liviano $\left(m_{\tilde{G}}=10^{-9} \mathrm{GeV}\right)$. Todos los términos trilineales son fijados a cero y las masas de los sleptones a $2.5 \mathrm{TeV}$. Las masas del Higgs liviano $(h)$ y el pseudoescalar $(A)$ se fijan a $m_{h}=126 \mathrm{GeV}$ y $m_{A}=2 \mathrm{TeV}$. La masa del $h$ se considera a partir del valor medido de la masa del Higgs observado en el año 2012. En modelos GGM de SUSY existen distintos mecanismos [115-119] para generar una masa del bosón de Higgs tan alta como este valor observado, sin cambiar la fenomenología de los modelos considerados. No se observó un efecto significativo en el espectro de masas variando el valor de la masa de Higgs en un rango de $\pm 10 \mathrm{GeV}$.

A partir de estos estudios se procedió a simular un conjunto de puntos de señal (grid) en el plano $\left(m_{\tilde{g}}, m_{\widetilde{\chi}_{1}^{0}}\right)$, variando los parámetros $M_{3}$ y $\mu$. El parámetro $M_{1}$ se ajustó, dependiendo del $\mu$ de forma de obtener las BR descriptas anteriormente. La grid cubre el espacio $150 \mathrm{GeV}<m_{\widetilde{\chi}_{1}^{0}}<1250 \mathrm{GeV}$ y $800 \mathrm{GeV}<m_{\widetilde{g}}<1450 \mathrm{GeV}$, con $m_{\widetilde{\chi}_{1}^{0}}<m_{\widetilde{g}}$ (ver Figura 6.4).

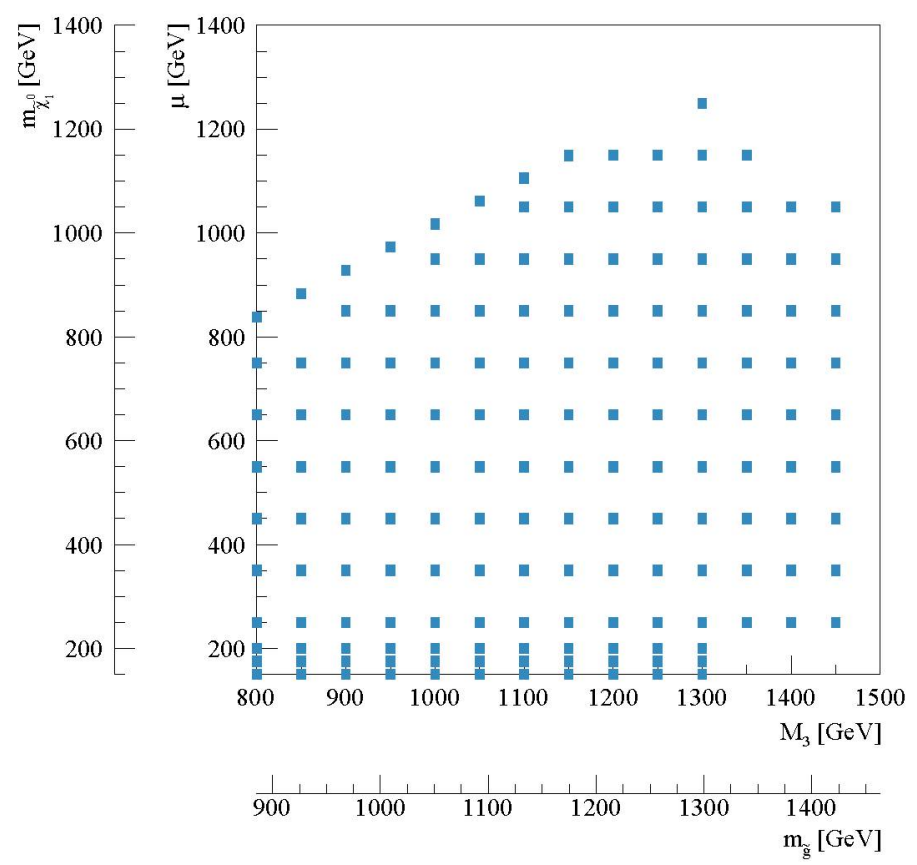

Figura 6.4: Conjunto de puntos (grid) de señal simulada para el presente análisis. Los cuadrados azules representan los distintos puntos de señal generados con distintos valores de $M_{3}$ y $\mu$. También puede verse la relación con las masas $m_{\widetilde{g}}$ y $m_{\widetilde{\chi}_{1}^{0}}$.

El espectro completo de masas y los correspondientes decaimientos fueron calculados a partir de estos parámetros utilizando Suspect (v2.41) [106], Sdecay (v1.3b) [107] y HdecAY (v3.4) [108], que forman parte del paquete Susyhit (v1.3) [105]. Algunos ejemplos del espectro de masas pueden verse en la Figura 6.5, para algunos puntos de la grid, $(885,146)$ y $(1463,1053)$.

Para cada uno de los 160 puntos de señal que constituyen la grid se generaron 5000 eventos, utilizando Herwig + + v2.5.2 [120] y el conjunto de PDFs CTEQ6L1 [111]. Se aplicó un filtro a nivel generador, que requería la presencia de al menos un fotón con $p_{\mathrm{T}}>100 \mathrm{GeV}$, para optimizar la generación, especialmente en los puntos donde el neutralino tiene menor masa. La eficiencia del filtro para todas las muestras simuladas puede verse en la Tabla 6.2. La simulación del detector ATLAS se realizó con la simulación rápida ATLFAST-II [114].

Las muestras de la grid mencionada anteriormente solo consideran la producción (fuerte) de pares de gluinos. Adicionalmente se simularon muestras de la producción electrodébil de gauginos, para las 

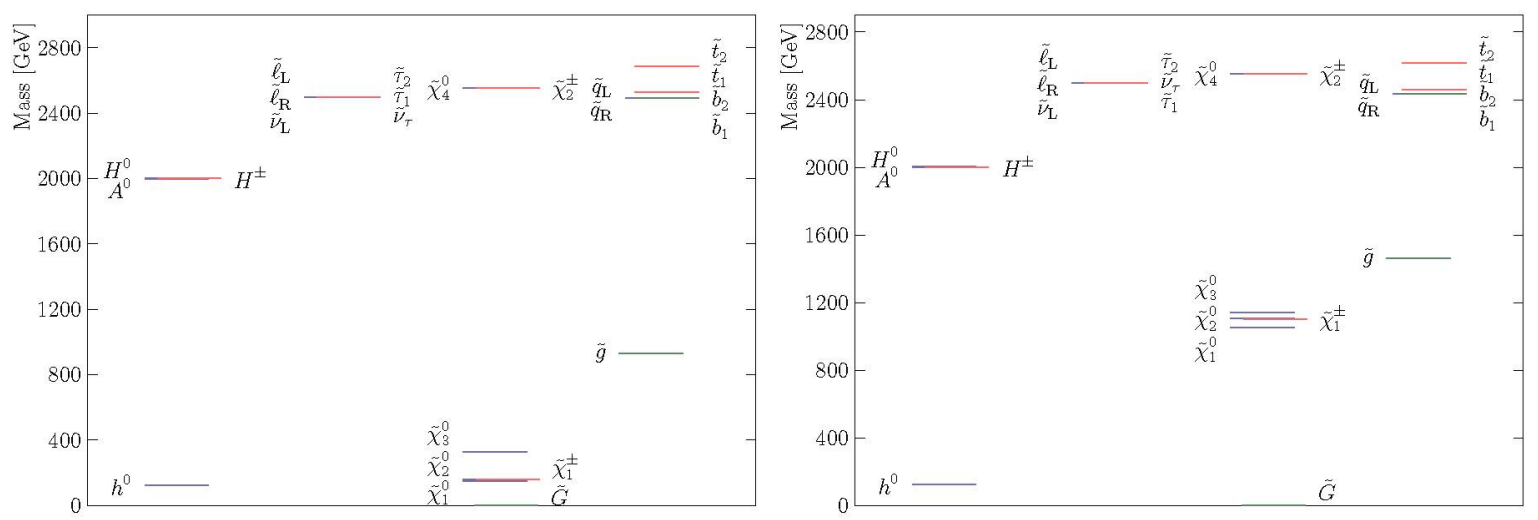

Figura 6.5: Espectro de masas de las partículas supersimétricas para dos puntos de la grid de señal. A la izquierda para $(885,146)$ y a la derecha para $(1463,1053)$, en el plano $\left(m_{\widetilde{g}}, m_{\widetilde{\chi}_{1}^{0}}\right)$.

distintas masas de neutralino consideradas en la grid, y utilizando la misma configuración. Como se detallará más adelante la sección eficaz de producción electrodébil es solo relevante, en el espacio de fase considerado, en la zona de baja masa de neutralino. Además el estado final en este caso estará caracterizado por una menor multiplicidad de jets, debido a la cadena de decaimiento más corta, y menor energía faltante. A pesar de que las regiones de señal fueron optimizadas para la producción de gluinos, ambas muestras son igualmente combinadas, en cada punto, a modo de completitud.

\subsubsection{Estudios a nivel generador}

Se realizaron algunos estudios para entender mejor el estado final y poder identificar las distintas regiones en el espacio de fase, lo cual facilitaría el proceso de optimización de las posibles regiones de señal. En la Figura 6.6 (izquierda) se presentan los distintos BR de los gluinos para todos los puntos de la grid. Resulta evidente que la cadena de decaimiento dominante varía en las distintas regiones de la grid, dependiendo de la masa del gluino y el neutralino. Se puede ver que el decaimiento dominante es a través de charginos, pero a medida que la masa del gluino y el neutralino se acercan, empieza a resultar más importante el decaimiento directo al neutralino más liviano, mientras que en los puntos más cercanos a la diagonal, el decaimiento estará dominado por $\widetilde{g} \rightarrow g \widetilde{G}$ el cual no corresponde al estado final deseado. Los diagramas de los distintos decaimientos posibles se encuentran en la Figura 6.7, de los cuales se pueden extraer varias conclusiones: en el caso de las cadenas largas a través de charginos, el estado final tendrá una mayor cantidad de jets. Si el decaimiento es directamente al $\widetilde{\chi}_{1}^{0}$ la multiplicidad de jets será menor y la energía del fotón será mayor al igual que la energía faltante.

En la Figura 6.6 (derecha) se presentan las BR de decaimiento del neutralino más liviano $\left(\widetilde{\chi}_{1}^{0}\right)$, compatibles con las esperadas a partir del modelo que se utilizará para el presente análisis (ecuaciones (6.1)-(6.3)). Estos valores varían en menos del $1 \%$ en los distintos puntos de la grid, salvo para neutralinos livianos $(<200 \mathrm{GeV})$ donde la producción de Higgs es altamente suprimida, mientras aumenta el decaimiento a $Z, y \operatorname{BR}\left(\widetilde{\chi}_{1}^{0} \rightarrow \gamma \widetilde{G}\right)$ empieza a caer llegando al $40 \%$.

\subsubsection{Sección eficaz de producción}

En el caso de procesos de producción de pares de gluinos o squarks, de los cuales existe el cálculo a NLL, la sección eficaz se toma a NLO en la constante de acoplamiento fuerte, y se incluye la suma de la emisión de gluones soft con precisión NLL, realizada utilizando NLL-FAST [121-126].

En el caso de la producción de otro tipo de procesos, como la producción electrodébil de partículas supersimétricas, se utilizan las secciones eficaces calculadas a NLO usando ProsPINo [57]. 
Tabla 6.1: Relación entre los parámetros libres del modelo $M_{3}$ y $\mu$ con las masas $m_{\widetilde{g}}$ (izquierda) y $m_{\widetilde{\chi}_{1}^{0}}$ (derecha), respectivamente. En la tabla de la derecha, para cada valor de $\mu$ también se puede observar el valor de $M_{1}$ utilizado para obtener los BR de decaimiento del $\widetilde{\chi}_{1}^{0}$ deseados.

\begin{tabular}{|c|c|c|c|c|}
\hline \multicolumn{2}{|c|}{$M_{3}[\mathrm{GeV}] \quad m_{\widetilde{g}}[\mathrm{GeV}]$} & \multirow[b]{2}{*}{$\mu[\mathrm{GeV}]$} & \multirow[b]{2}{*}{$M_{1}[\mathrm{GeV}]$} & \multirow[b]{2}{*}{$m_{\widetilde{\chi}_{1}^{0}}[\mathrm{GeV}]$} \\
\hline 800 & 885.5 & & & \\
\hline 850 & 931.7 & 150 & 300 & 147.0 \\
\hline 900 & 977.6 & 175 & 270 & 168.3 \\
\hline 950 & 1023.1 & 200 & 267 & 190.3 \\
\hline 1000 & 1068.3 & 250 & 288 & 235.8 \\
\hline 1050 & 1113.3 & 350 & 365 & 332.4 \\
\hline 1100 & 1157.9 & 450 & 456 & 433.2 \\
\hline 1150 & 1202.3 & 550 & 551 & 535.6 \\
\hline 1200 & 1246.4 & 650 & 647 & 638.3 \\
\hline 1250 & 1290.3 & 750 & 745 & 742.0 \\
\hline 1300 & 1333.9 & 838 & 837 & 836.4 \\
\hline 1350 & 1377.3 & 850 & 845 & 846.7 \\
\hline 1400 & 1420.5 & 883 & 882 & 883.7 \\
\hline 1450 & 1463.4 & 928 & 926 & 930.2 \\
\hline & & 950 & 942 & 949.6 \\
\hline & & 973 & 970 & 976.6 \\
\hline & & 1017 & 1015 & 1023.4 \\
\hline & & 1050 & 1040 & 1053.0 \\
\hline & & 1062 & 1058 & 1068.9 \\
\hline & & 1106 & 1102 & 1114.8 \\
\hline & & 1149 & 1145 & 1160.0 \\
\hline & & 1150 & 1140 & 1157.5 \\
\hline & & 1250 & 1238 & 1260.6 \\
\hline
\end{tabular}

Las incertezas debidas a la elección de la escala de renormalización y factorización como también aquellas asociadas a la PDF, son obtenidas utilizando NLL-FAsT o calculadas con ProsPINO. A fin de combinar todas estas predicciones y obtener una incerteza total, se siguen las recomendaciones PDF4LHC [127]. Para esto se define la envolvente de las predicciones a la sección eficaz usando el intervalo a $68 \% \mathrm{CL}$ del conjunto de PDFs CTEQ (incluyendo la incerteza en $\alpha_{s}$ ) y MSTW, junto con las variaciones de las escalas. La sección eficaz nominal se obtiene usando el punto medio de la envolvente y la incerteza como la mitad del ancho de la misma. Matemáticamente si PDF CTEQ $^{ \pm}$ $\mu_{\mathrm{CTEQ}}^{ \pm}$son las variaciones en $\pm 1 \sigma$ de las PDF CTEQ, PDF ${ }_{\text {MSTw }}^{ \pm} \mu_{\mathrm{MSTW}}^{ \pm}$son las variaciones en $\pm 1 \sigma$ en la PDF MSTW, y $\alpha_{s}^{ \pm}$son las correspondientes incertezas de la constante de acoplamiento fuerte, entonces,

$$
\begin{aligned}
& \mathrm{CTEQ}^{ \pm}=\sqrt{\left(\mathrm{PDF}_{\mathrm{CTEQ}}^{ \pm}\right)^{2}+\left(\mu_{\mathrm{CTEQ}}^{ \pm}\right)^{2}+\left(\alpha_{s}^{ \pm}\right)^{2}} \\
& \mathrm{MSTW}^{ \pm}=\sqrt{\left(\mathrm{PDF}_{\mathrm{MSTW}}^{ \pm}\right)^{2}+\left(\mu_{\mathrm{MSTW}}^{ \pm}\right)^{2}}
\end{aligned}
$$

Los correspondientes extremos por arriba y abajo de la envolvente pueden calcularse a partir de estos: 
Tabla 6.2: Eficiencia del filtro a nivel generador [\%] para los puntos de señal simulados.

\begin{tabular}{|c|c|c|c|c|c|c|c|c|c|c|c|c|c|c|}
\hline \multirow[b]{2}{*}{$\mu[\mathrm{GeV}]$} & \multicolumn{14}{|c|}{$M_{3}[\mathrm{GeV}]$} \\
\hline & 800 & 850 & 900 & 950 & 1000 & 1050 & 1100 & 1150 & 1200 & 1250 & 1300 & 1350 & 1400 & 1450 \\
\hline 150 & 39.54 & 39.8 & 41.9 & 42.6 & 43.0 & 44.2 & 45.5 & 46.3 & 47.1 & 48.3 & 49.1 & & & \\
\hline 175 & 44.55 & 44.6 & 46.5 & 47.5 & 47.8 & 48.9 & 50.3 & 51.4 & 51.5 & 52.4 & 53.3 & & & \\
\hline 200 & 47.66 & 48.4 & 50.1 & 50.6 & 52.0 & 52.8 & 53.8 & 55.0 & 55.0 & 56.2 & 56.0 & & & \\
\hline 250 & 55.09 & 56.0 & 56.1 & 57.1 & 56.7 & 58.0 & 58.9 & 59.5 & 60.5 & 60.7 & 61.2 & 62.1 & 62.2 & 63.9 \\
\hline 350 & 65.82 & 66.1 & 64.6 & 65.8 & 66.4 & 66.7 & 66.5 & 67.7 & 67.4 & 67.6 & 67.8 & 68.0 & 69.2 & 68.4 \\
\hline 450 & 71.29 & 71.4 & 71.4 & 71.8 & 72.1 & 72.5 & 72.0 & 72.6 & 72.1 & 72.5 & 73.4 & 72.9 & 72.2 & 73.4 \\
\hline 550 & 73.08 & 72.5 & 73.3 & 73.7 & 74.6 & 74.3 & 75.0 & 74.8 & 74.2 & 74.7 & 75.6 & 75.7 & 74.9 & 75.3 \\
\hline 650 & 69.78 & 72.0 & 73.2 & 74.1 & 75.6 & 76.2 & 75.9 & 75.8 & 77.0 & 77.0 & 76.2 & 76.7 & 77.1 & 76.6 \\
\hline 750 & 47.69 & 60.2 & 66.9 & 70.4 & 73.4 & 74.0 & 75.9 & 76.5 & 77.1 & 77.3 & 76.3 & 77.9 & 77.2 & 77.0 \\
\hline 838 & 9.0 & & & & & & & & & & & & & \\
\hline 883 & & 9.1 & & & & & & & & & & & & \\
\hline 850 & & & 34.2 & 50.7 & 60.1 & 66.4 & 70.4 & 73.5 & 73.6 & 75.4 & 76.6 & 77.1 & 78.2 & 76.8 \\
\hline 928 & & & 9.4 & & & & & & & & & & & \\
\hline 950 & & & & & 25.4 & 41.4 & 54.0 & 62.2 & 66.8 & 70.9 & 75.2 & 75.9 & 78.1 & 77.2 \\
\hline 973 & & & & 10.0 & & & & & & & & & & \\
\hline 1017 & & & & & 10.3 & & & & & & & & & \\
\hline 1050 & & & & & & & 19.2 & 31.6 & 45.2 & 56.8 & 62.7 & 68.9 & 73.1 & 75.6 \\
\hline 1062 & & & & & & 11.1 & & & & & & & & \\
\hline 1106 & & & & & & & 11.7 & & & & & & & \\
\hline 1149 & & & & & & & & 12.6 & & & & & & \\
\hline 1150 & & & & & & & & & 16.1 & 24.9 & 36.9 & 49.0 & & \\
\hline 1250 & & & & & & & & & & & 16.1 & & & \\
\hline
\end{tabular}
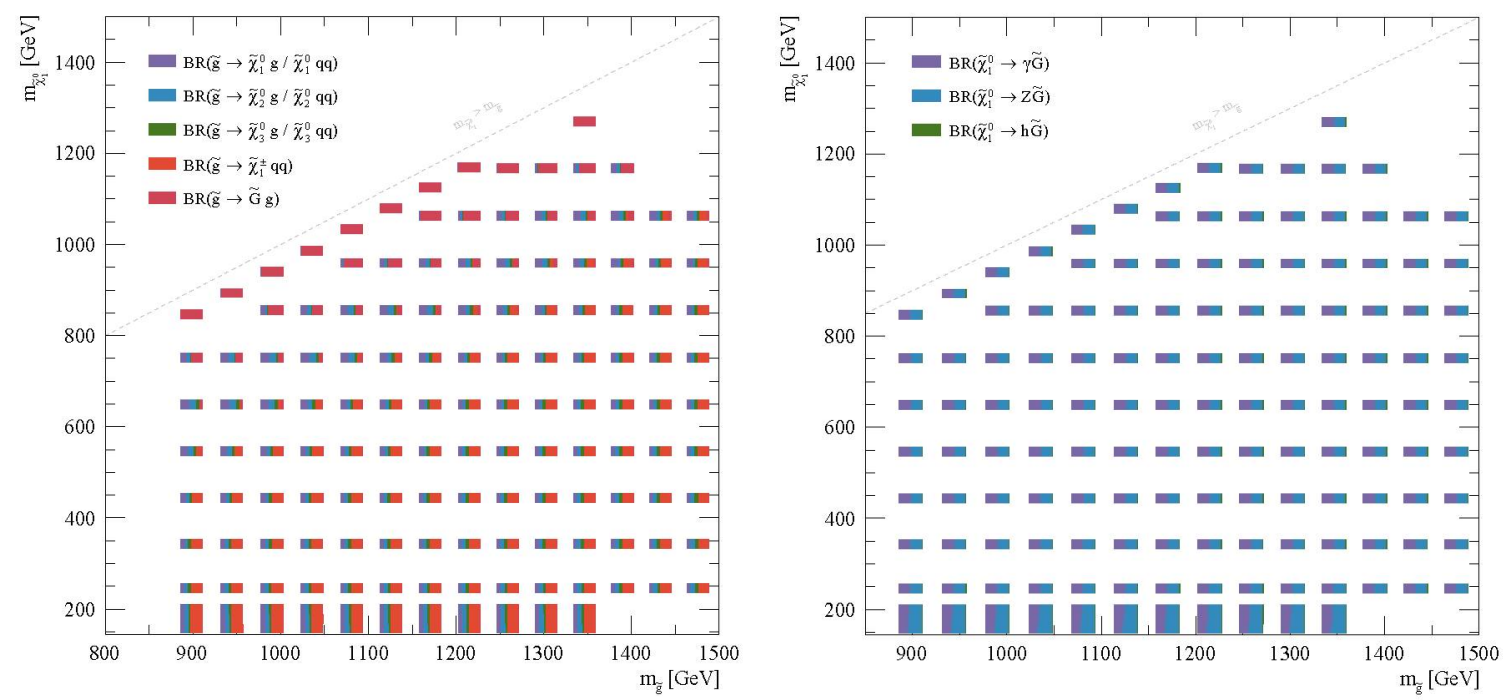

Figura 6.6: Tasas de decaimiento (BR) de los gluinos (izquierda) y de los neutralinos NLSP (derecha) para los distintos puntos de la grid en el plano $\left(m_{\tilde{g}}, m_{\widetilde{\chi}_{1}^{0}}\right)$. Para cada punto de señal, la fracción del rectángulo de cada color representa el BR a cada uno de los posibles estados finales.

$$
\begin{array}{ll}
U=\operatorname{máx}\left(\mathrm{CTEQ}^{\text {nom }}+\mathrm{CTEQ}^{+},\right. & \left.\mathrm{MSTW}^{\text {nom }}+\mathrm{MSTW}^{+}\right) \\
L=\operatorname{mín}\left(\mathrm{CTEQ}^{\text {nom }}-\mathrm{CTEQ}^{-},\right. & \left.\mathrm{MSTW}^{\text {nom }}-\mathrm{MSTW}^{-}\right)
\end{array}
$$

y la sección eficaz final $(\sigma)$ y su incerteza simétrica $(\Delta \sigma)$ se obtienen como:

$$
\sigma=(U+L) / 2, \quad \Delta \sigma=(U-L) / 2
$$

En la Tabla 6.3 puede verse la sección eficaz de producción de pares de gluinos para los distintos puntos de la grid de señal generada. La sección eficaz de producción electrodébil de neutralinos/charginos se encuentra en la Tabla 6.4. En la Figura 6.8 también se encuentra graficada la sección eficaz como 

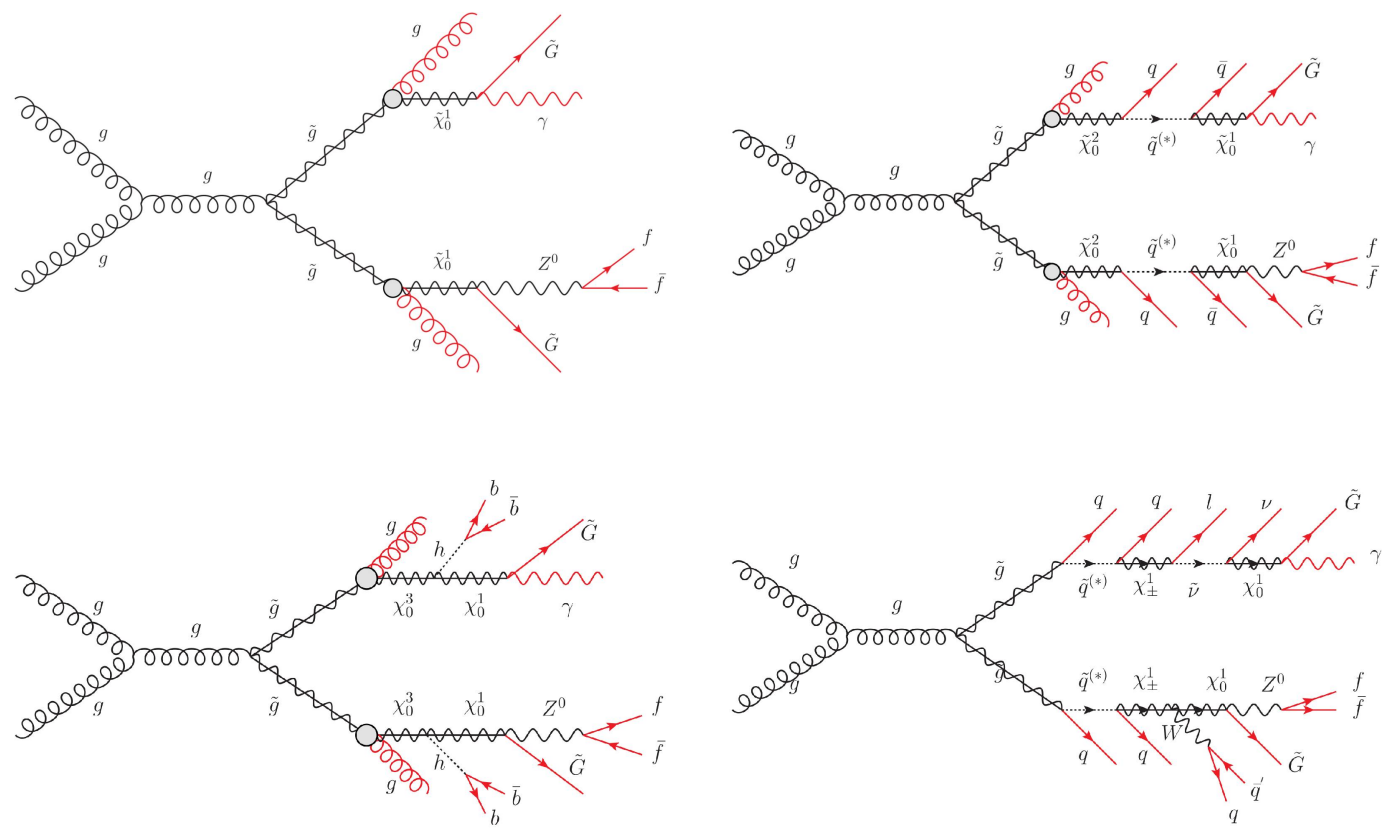

Figura 6.7: Diagramas de Feynman ilustrando las posibles cadenas de decaimiento que dan lugar el estado final considerado.

función de la masa de las partículas producidas, mientras que en la Figura 6.9 se puede apreciar la fracción en la producción electrodébil comparada con la producción total.

Tabla 6.3: Sección eficaz a NLO+NLL para la producción de gluinos para los distintos puntos de la grid de señal. La última columna es la incerteza teórica.

\begin{tabular}{ccrr}
\hline$M_{3}[\mathrm{GeV}]$ & $m_{\widetilde{g}}[\mathrm{GeV}]$ & $\sigma(\mathrm{NLO}+\mathrm{NLL})[\mathrm{pb}]$ & Incerteza Total $[\%]$ \\
\hline 800 & 885.5 & 0.06905 & 22.5 \\
850 & 931.7 & 0.04492 & 23.8 \\
900 & 977.6 & 0.02973 & 25.2 \\
950 & 1023.1 & 0.01983 & 26.5 \\
1000 & 1068.3 & 0.01341 & 27.7 \\
1050 & 1113.3 & 0.00910 & 29.0 \\
1100 & 1157.9 & 0.00628 & 30.4 \\
1150 & 1202.3 & 0.00432 & 32.0 \\
1200 & 1246.4 & 0.00301 & 33.7 \\
1250 & 1290.3 & 0.00210 & 35.2 \\
1300 & 1333.9 & 0.00148 & 36.7 \\
1350 & 1377.3 & 0.00105 & 38.2 \\
1400 & 1420.5 & 0.00074 & 39.8 \\
1450 & 1463.4 & 0.00053 & 41.5 \\
\hline
\end{tabular}


Tabla 6.4: Sección eficaz total a NLO para la producción electrodébil de neutralinos y charginos para los distintos puntos de la grid de señal. La última columna es la incerteza teórica.

\begin{tabular}{ccrr}
\hline$\mu[\mathrm{GeV}]$ & $m_{\tilde{\chi}_{1}^{0}}[\mathrm{GeV}]$ & $\sigma(\mathrm{NLO})[\mathrm{pb}]$ & Incerteza total $[\%]$ \\
\hline 150 & 147.0 & 2.68 & 6.3 \\
175 & 168.3 & 1.42 & 6.7 \\
200 & 190.3 & 0.84 & 6.9 \\
250 & 235.8 & 0.28 & 6.4 \\
350 & 332.4 & 0.050 & 7.0 \\
450 & 433.2 & 0.013 & 7.6 \\
550 & 535.6 & $4.1 \cdot 10^{-3}$ & 8.0 \\
650 & 638.3 & $1.4 \cdot 10^{-3}$ & 8.5 \\
750 & 742.0 & $5.3 \cdot 10^{-4}$ & 8.9 \\
850 & 846.7 & $2.1 \cdot 10^{-4}$ & 9.3 \\
950 & 949.6 & $8.57 \cdot 10^{-5}$ & 10.3 \\
1050 & 1053.0 & $3.56 \cdot 10^{-5}$ & 11.0 \\
1150 & 1157.5 & $1.55 \cdot 10^{-5}$ & 13.3 \\
1250 & 1260.6 & $6.67 \cdot 10^{-6}$ & 16.9 \\
\hline
\end{tabular}
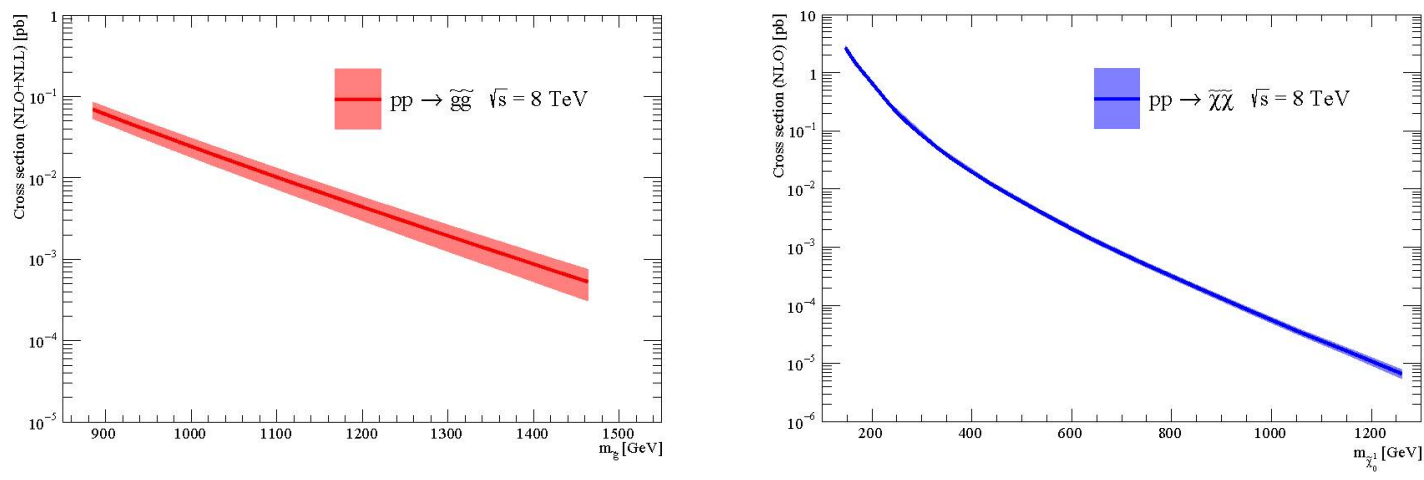

Figura 6.8: Sección eficaz de producción de pares de gluinos como función de $m_{\widetilde{g}}$ (izquierda) y de pares de neutralinos/charginos como función de $m_{\widetilde{\chi}_{1}^{0}}$ (derecha).
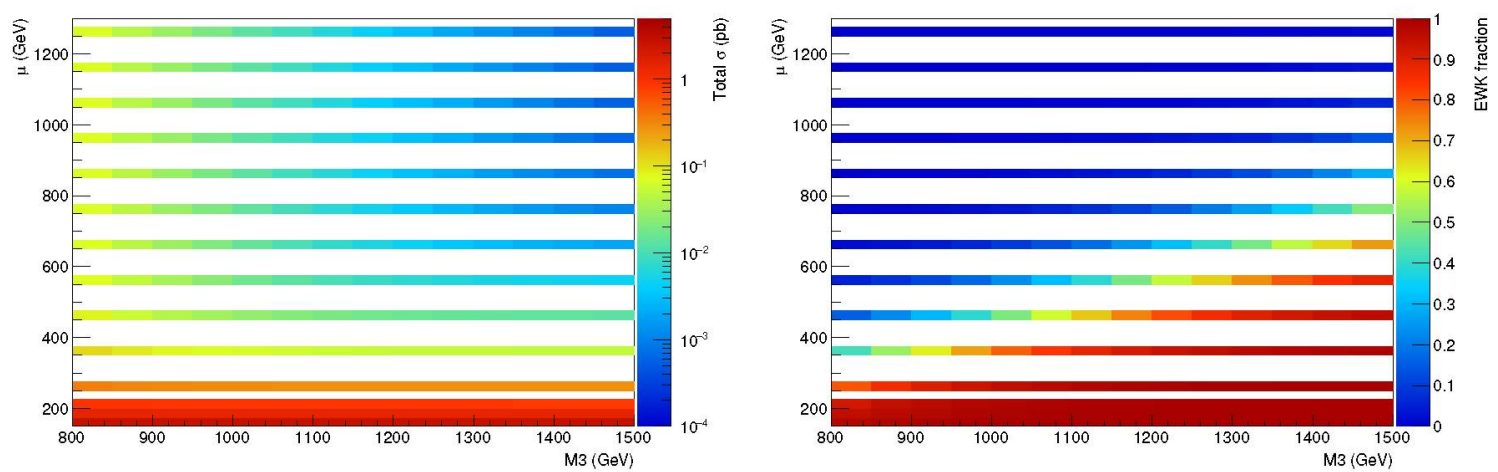

Figura 6.9: Sección eficaz total (izquierda) y fracción relativa de producción electrodébil (derecha). 


\subsection{Simulación de los fondos del SM}

Las muestras de los procesos de fondo contaminante simuladas con MC utilizadas en este análisis se describen a continuación. Como se discutirá en la capítulo 8, la contaminación de fotones mal identificados provenientes de jets y electrones es estimada con métodos basados en datos. Sin embargo, las muestras MC también han sido consideradas en estos casos para los estudios de optimización y la evaluación de las incertezas sistemáticas.

\subsection{1. $W / Z+\gamma$}

Se espera que la producción de $W \gamma$ y $Z \gamma$ sea un fondo importante para esta búsqueda. Ambas muestras fueron generadas usando el generador de eventos SHERPA v1.4.1 [128] y usando las funciones de distribución partónica CT10. La combinación de los elementos de matriz con las lluvias partónicas es realizada de acuerdo a un procedimiento mejorado CKKW $[129,130]$. Un filtro a nivel generador es aplicado requiriendo al menos un fotón con $p_{\mathrm{T}}>80(70) \mathrm{GeV}$ en el estado final de las muestras de $W \gamma$ $(Z \gamma)$. Todos los decaimientos leptónicos del bosón $Z$ fueron considerados, incluyendo el decaimiento invisible $Z \rightarrow \nu \nu$. También se tuvo en cuenta un muestra de $V(\rightarrow q q)+\gamma(V=W, Z / \gamma *)$ debido a que cierta energía faltante real puede ser producida en el caso de decaimientos de sabores pesados.

Tabla 6.5: Muestras de $\mathrm{W} / \mathrm{Z}+\gamma$. La sección eficaz a LO se especifica para cada modo de decaimiento, al igual que los factores $k$, y las eficiencias del filtro. La última columna contiene la luminosidad integrada correspondiente a la estadística total de cada muestra.

\begin{tabular}{lcrrrr}
\hline Proceso & Generador & $\sigma[\mathrm{pb}]$ & Factor- $k$ & Eficiencia & $L\left[\mathrm{fb}^{-1}\right]$ \\
\hline $\mathrm{W}(\rightarrow e \nu)+\gamma$ & SHERPA & 0.7193 & 1.0 & 1.0 & 695.16 \\
$\mathrm{~W}(\rightarrow \mu \nu)+\gamma$ & SHERPA & 0.7178 & 1.0 & 1.0 & 696.56 \\
$\mathrm{~W}(\rightarrow \tau \nu)+\gamma$ & SHERPA & 0.7199 & 1.0 & 1.0 & 694.57 \\
$\mathrm{Z}(\rightarrow e e)+\gamma$ & SHERPA & 0.1861 & 1.0 & 1.0 & 1069.53 \\
$\mathrm{Z}(\rightarrow \mu \mu)+\gamma$ & SHERPA & 0.1858 & 1.0 & 1.0 & 1076.71 \\
$\mathrm{Z}(\rightarrow \tau \tau)+\gamma$ & SHERPA & 0.1858 & 1.0 & 1.0 & 1076.19 \\
$Z(\rightarrow \nu \nu) \gamma$ & SHERPA & 0.7625 & 1.0 & 1.0 & 655.74 \\
$V(\rightarrow q q) \gamma$ & SHERPA & 6.756 & 1.0 & 1.0 & 89.0 \\
\hline
\end{tabular}

\subsection{2. $W / Z+$ jets}

Se espera que la producción de $\mathrm{W}^{ \pm}$y bosones $Z$ en asociación con jets contribuya a los fondos contaminantes de esta búsqueda, con los fotones provenientes de electrones y jets mal identificados. Especialmente para los segundos, esta contaminación no está bien descripta por el MC. Por esta razón se utilizan métodos basados en datos para estimar su contribución en las diferentes regiones de señal y control, como se describe en el capítulo 8. Varias muestras de MC fueron consideradas para validar los métodos.

Como se describe en el capítulo 7 la selección de señal involucra muchos jets en el estado final, razón por lo que es importante modelar los estados final multipartónicos de forma adecuada. Con esto en mente, el generador de eventos ALPGEn (v 2.14) fue utilizado, incluyendo los efectos EWK y QCD a LO para los procesos de interacción fuerte multipartónicos. Se utilizó Herwig v6.5.2 para la simulación de las lluvias y los procesos de fragmentación y Jimmy para la simulación de los eventos subyacentes. 
Las funciones de densidad partónica utilizadas fueron las CTEQ6L1. La normalización a la luminosidad integrada acumulada se realizó a partir de la sección eficaz mostrada en la Tabla 6.6 usando cálculos QCD a NNLO del programa FEWZ [131]. En cada caso los mismos factores de normalización fueron aplicados a los elementos de matriz de Alpgen.

Finalmente, se realizó la remoción de eventos para evitar el conteo doble de eventos que ya fueron tenidos en cuenta en las muestras de $\mathrm{Z} \gamma \mathrm{y} \mathrm{W} \gamma$. Para esto, los eventos de $W(Z)+$ jets con fotones con $p_{\mathrm{T}}>80(70) \mathrm{GeV}$ y $\Delta R(\ell / q / g, \gamma)>0.1$ fueron removidos de las muestras.

Tabla 6.6: Muestras de $W / Z+$ jets. Se detallan la sección eficaz a LO para cada modo de decaimiento, los factores $k$ (para la normalización NLO) y las eficiencias del filtro, así como también la luminosidad integrada correspondiente a la estadística total de cada muestra.

\begin{tabular}{|c|c|c|c|c|c|}
\hline Proceso & Generador & $\sigma[\mathrm{pb}]$ & Factor- $k$ & Eficiencia & $L\left[\mathrm{fb}^{-1}\right]$ \\
\hline$Z \rightarrow e e$ & ALPGEN $+J_{\text {IMMY }}$ & 711.77 & 1.23 & 1.00 & 7.548 \\
\hline$Z \rightarrow e e(+1$ jet $)$ & ALPGEN $+J_{\text {IMMY }}$ & 155.17 & 1.23 & 1.00 & 6.994 \\
\hline$Z \rightarrow e e(+2$ jets $)$ & ALPGEN $+J_{\text {IMMY }}$ & 48.745 & 1.23 & 1.00 & 6.746 \\
\hline$Z \rightarrow e e(+3$ jets $)$ & ALPGEN $+J_{\text {IMMY }}$ & 14.225 & 1.23 & 1.00 & 6.286 \\
\hline$Z \rightarrow e e(+4$ jets $)$ & ALPGEN+J IMMY & 3.7595 & 1.23 & 1.00 & 6.487 \\
\hline$Z \rightarrow e e(+5$ jets $)$ & ALPGEN $+J_{\text {IMM }}$ & 1.0945 & 1.23 & 1.00 & 7.428 \\
\hline$Z \rightarrow \mu \mu$ & ALPGEN + J IMMY & 712.11 & 1.23 & 1.00 & 7.557 \\
\hline$Z \rightarrow \mu \mu(+1$ jet $)$ & ALPGEN +J IMMY & 154.77 & 1.23 & 1.00 & 7.011 \\
\hline$Z \rightarrow \mu \mu(+2$ jets $)$ & ALPGEN + J IMMY & 48.912 & 1.23 & 1.00 & 6.731 \\
\hline$Z \rightarrow \mu \mu(+3$ jets $)$ & ALPGEN + JIMMY & 14.226 & 1.23 & 1.00 & 6.286 \\
\hline$Z \rightarrow \mu \mu(+4$ jets $)$ & ALPGEN + J IMMY & 3.7838 & 1.23 & 1.00 & 6.445 \\
\hline$Z \rightarrow \mu \mu(+5$ jets $)$ & ALPGEN + J IMMY & 1.1148 & 1.23 & 1.00 & 7.292 \\
\hline$Z \rightarrow \tau \tau$ & ALPGEN $+J_{\text {IMMY }}$ & 711.81 & 1.23 & 1.00 & 7.560 \\
\hline$Z \rightarrow \tau \tau(+1$ jet $)$ & ALPGEN+J IMMY & 155.13 & 1.23 & 1.00 & 6.996 \\
\hline$Z \rightarrow \tau \tau(+2$ jets $)$ & ALPGEN $+J_{\text {IMMY }}$ & 48.804 & 1.23 & 1.00 & 6.746 \\
\hline$Z \rightarrow \tau \tau(+3$ jets $)$ & ALPGEN+JIMMY & 14.160 & 1.23 & 1.00 & 6.315 \\
\hline$Z \rightarrow \tau \tau(+4$ jets $)$ & ALPGEN $+J_{\text {IMMY }}$ & 3.7744 & 1.23 & 1.00 & 6.462 \\
\hline$Z \rightarrow \tau \tau(+5$ jets $)$ & ALPGEN + JIMMY & 1.1163 & 1.23 & 1.00 & 7.283 \\
\hline$W \rightarrow e \nu$ & ALPGEN $+J_{\text {IMMY }}$ & 8037.10 & 1.186 & 1.00 & 0.362 \\
\hline$W \rightarrow e \nu(+1$ jet $)$ & ALPGEN + JIMMY & 1579.20 & 1.186 & 1.00 & 1.334 \\
\hline$W \rightarrow e \nu(+2$ jets $)$ & ALPGEN $+J_{\text {IMMY }}$ & 477.20 & 1.186 & 1.00 & 6.661 \\
\hline$W \rightarrow e \nu(+3$ jets $)$ & ALPGEN $+J_{\text {IMMM }}$ & 133.93 & 1.186 & 1.00 & 6.358 \\
\hline$W \rightarrow e \nu(+4$ jets $)$ & ALPGEN $+J_{\text {IMMY }}$ & 35.62 & 1.186 & 1.00 & 5.917 \\
\hline$W \rightarrow e \nu(+5$ jets $)$ & ALPGEN+J IMMY & 10.55 & 1.186 & 1.00 & 5.592 \\
\hline$W \rightarrow \mu \nu$ & ALPGEN $+J_{\text {IMMY }}$ & 8040.00 & 1.186 & 1.00 & 0.363 \\
\hline$W \rightarrow \mu \nu(+1$ jet $)$ & ALPGEN + JIMMY & 1580.30 & 1.186 & 1.00 & 1.333 \\
\hline$W \rightarrow \mu \nu(+2$ jets $)$ & ALPGEN + J IMMY & 477.50 & 1.186 & 1.00 & 6.656 \\
\hline$W \rightarrow \mu \nu(+3$ jets $)$ & ALPGEN $+J_{\text {IMMY }}$ & 133.94 & 1.186 & 1.00 & 6.357 \\
\hline$W \rightarrow \mu \nu(+4$ jets $)$ & ALPGEN $+J_{\text {IMMY }}$ & 35.64 & 1.186 & 1.00 & 6.033 \\
\hline$W \rightarrow \mu \nu(+5$ jets $)$ & ALPGEN $+J_{\text {IMMY }}$ & 10.57 & 1.186 & 1.00 & 1.595 \\
\hline$W \rightarrow \tau \nu$ & ALPGEN $+J_{\text {IMMY }}$ & 8035.80 & 1.186 & 1.00 & 0.353 \\
\hline$W \rightarrow \tau \nu(+1$ jet $)$ & ALPGEN $+J_{\text {IMMY }}$ & 1579.80 & 1.186 & 1.00 & 1.307 \\
\hline$W \rightarrow \tau \nu(+2$ jets $)$ & ALPGEN + JIMMY & 477.55 & 1.186 & 1.00 & 6.567 \\
\hline$W \rightarrow \tau \nu(+3$ jets $)$ & ALPGEN $+J_{\text {IMMY }}$ & 133.79 & 1.186 & 1.00 & 6.365 \\
\hline$W \rightarrow \tau \nu(+4$ jets $)$ & ALPGEN + JIMMY & 35.58 & 1.186 & 1.00 & 5.921 \\
\hline$W \rightarrow \tau \nu(+5$ jets $)$ & ALPGEN $+J_{\text {IMMY }}$ & 10.54 & 1.186 & 1.00 & 5.199 \\
\hline
\end{tabular}




\subsubsection{Pares de tops $(+\gamma)$}

Otro fondo importante para este análisis es el $t \bar{t} \gamma$. Esta muestra fue generada utilizando MADGRAPH [132] y la PDF CTEQ6L1. Pythia6 [133] fue usado para la simulación de las lluvias partónicas, fragmentación y eventos subyacentes. La radiación de fotones fue agregadas utilizando Photos [134], y los decaimientos de los leptones tau con Tauola [135]. Se requirió que los fotones a nivel generador tengan un $p_{\mathrm{T}}>80 \mathrm{GeV}$. Para evitar efectos cinemáticos introducidos por el filtro, el corte en el $p_{\mathrm{T}}$ del fotón en la muestra reconstruida se aumentó a $95 \mathrm{GeV}$. Se utilizó un factor- $k$ de $1.9 \pm 0.4[136,137]$ para tener en cuenta efectos de mayor orden en teoría de perturbaciones. Los detalles de la simulación se encuentran en la Tabla 6.7. Se utilizaron además algunas muestras a nivel generador como variaciones para calcular las incertezas sistemáticas como se explica en la sección 9.2.2.

La producción de $t \bar{t}$, donde los electrones o los jets son mal identificados como fotones es una fuente de fondo que debe ser considerado. Aunque ambas contaminaciones fueron estimadas a partir de los datos, se utilizaron eventos simulados en la etapa de optimización y para realizar chequeos de los métodos utilizados. La muestra MC fue generada utilizando Powheg [138-140] con la lluvia partónica y fragmentación hecha por Pyтhia. Para el UE se utilizó Perugia 2011C con el conjunto de PDFs CTEQ6L1 LO. La radiación de fotones adicional fue agregada con PHotos [134].

Por último, se realizó la remoción de los eventos con un fotón real con $p_{\mathrm{T}}>95 \mathrm{GeV}$ y $\Delta R(\ell / g / q, \gamma)>$ 0.1 de la muestra de $t \bar{t}$ para evitar la superposición con los eventos considerados en las muestras de $t \bar{t} \gamma$.

Tabla 6.7: Muestras de $t \bar{t} \gamma$. Se detallan la sección eficaz a LO para cada modo de decaimiento, los factores $k$ (para la normalización NLO) y las eficiencias del filtro, así como también la luminosidad integrada correspondiente a la estadística total de cada muestra.

\begin{tabular}{lcrrrr}
\hline Proceso & Generador & $\sigma[\mathrm{pb}]$ & Factor- $k$ & Eficiencia & $L\left[\mathrm{fb}^{-1}\right]$ \\
\hline$t \bar{t}$ & PowhEG+PYthiA & 253.00 & 1.00 & 0.543 & 580 \\
\hline$t \bar{t} \gamma$ (lep) & MadGRAPH & 0.09873 & 1.90 & 1.00 & 1066.2 \\
$t \bar{t} \gamma$ (had) & MADGRAPH & 0.068599 & 1.90 & 1.00 & 1534.5 \\
\hline
\end{tabular}

\subsubsection{Top $(+\gamma)$}

La producción de un quark top con un fotón asociado fue generado utilizando WHIZARD 2.1.1 $[141,142]$. El fotón extra puede estar tanto en la producción del top como en los decaimientos sucesivos. Sin embargo, la producción y el decaimiento son tratados de forma separada, por lo que los efectos de interferencia pueden ser ignorados. Para las lluvias partónicas y la fragmentación fue utilizado Pythia [133]. La radiación de fotones fue agregada con Photos [134], y los decaimientos de los leptones tau con TAUOLA [135].

La producción de tops resulta en un fondo poco importante para este análisis, aunque de mayor importancia para las regiones de control y validación. La producción $W t$ fue generada utilizando POWHEG, incluyendo correcciones a NLO en QCD. La lluvia partónica y la fragmentación fue simulada utilizando Pythia (con P2011). Se utilizó el conjunto de PDFs CT10. Las muestras fueron normalizadas con la sección eficaz calculada en [143]. 
Tabla 6.8: Muestras de quark top $+\gamma$. La sección eficaz a NNLO, eficiencia del filtro, y luminosidad integrada correspondiente a la estadística total de cada muestra están detalladas en la tabla.

\begin{tabular}{|c|c|c|c|c|}
\hline Proceso & Generador & $\sigma[\mathrm{pb}]$ & Eficiencia & $L\left[\mathrm{fb}^{-1}\right]$ \\
\hline top $+\gamma$ (en producción) & WIZHARD + PYTHIA & 0.187298 & 0.121980 & 4810 \\
\hline top $+\gamma($ en decaimiento $)$ & WIZHARD + PYTHIA & 0.313866 & 0.012927 & 4930 \\
\hline$t W($ lep $)+\gamma$ (en producción $)$ & WIZHARD + PYTHIA & 0.01292 & 0.16437 & 4710 \\
\hline$t W($ lep $)+\gamma($ en decaimiento del $t)$ & WIZHARD + PYTHIA & 0.01454 & 0.02875 & 12000 \\
\hline$t W($ lep $)+\gamma($ en decaimiento del $W)$ & WIZHARD+PYTHIA & 0.01041 & 0.07549 & 6370 \\
\hline$t($ lep $) W($ had $)+\gamma($ en producción $)$ & WIZHARD + PYTHIA & 0.02583 & 0.16244 & 4770 \\
\hline$t($ lep $) W($ had $)+\gamma($ en decaimiento del $t)$ & WiZHARD + PYTHIA & 0.02908 & 0.02761 & 6230 \\
\hline$t($ lep $) W($ had $)+\gamma($ en decaimiento del $W)$ & WIZHARD + PYTHIA & 0.01159 & 0.06471 & 6660 \\
\hline$t$ (had) $W($ lep $)+\gamma($ en producción $)$ & WIZHARD + PYTHIA & 0.02582 & 0.16178 & 4790 \\
\hline$t($ had $) W($ lep $)+\gamma($ en decaimiento del $t)$ & WIZHARD + PYTHIA & 0.02013 & 0.04198 & 5920 \\
\hline$t($ had $) W($ lep $)+\gamma($ en decaimiento del $W)$ & WIZHARD+PYTHIA & 0.02079 & 0.07574 & 3180 \\
\hline
\end{tabular}

\subsection{5. $\gamma+$ jets y multijets}

La contaminación debido a la producción QCD de fotones directos y multijets, es en todos los casos el resultado de eventos patológicos (jets mal identificados como fotones, y jets o fotones mal reconstruidos dejando una alta cantidad de energía faltante). Sin embargo, no se espera que sea un fondo dominante en el espacio de fase explorado en este análisis. La contribución de eventos con jets mal identificados como fotones se estima a partir de los datos en la sección 8.4.

Las muestras de multijets listadas en la Tabla 6.9 fueron utilizadas para el proceso de optimización y estudios de sensibilidad preliminares. La producción de fotones directos fue simulada con SHERPA v1.4.1 [128], con hasta cuatro partones en la ME+PS y usando el conjunto de PDFs CT10. El espectro inclusivo se dividió en diversas muestras con diferentes umbrales de $p_{\mathrm{T}}$ del fotón para optimizar la generación de eventos.

Muestras alternativas fueron utilizadas para la estimación de las incertezas sistemáticas, generadas con Pythia8 (usando CTEQ6L1) y ALPGEn v2.14 (con la misma configuración que los eventos de $W / Z+$ jets descriptos en sección 6.3.2). Los detalles pueden verse en la Tabla 6.9 .

\subsubsection{Diboson}

Los procesos de diboson (WW, WZ, y ZZ) fueron generados utilizando SHERPA y usando la PDF CT10, con la sección eficaz provista por MCFM [144]. Solo los decaimientos leptónicos para ambos bosones fueron considerados. 
Tabla 6.9: Muestras de QCD $\gamma+$ jets y multijet utilizadas en este análisis. La sección eficaz a LO para cada modo de decaimiento, y las eficiencias del filtro están detalladas, así como también la luminosidad integrada correspondiente a la estadística total de cada muestra.

\begin{tabular}{lcrrr}
\hline Proceso & Generador & $\sigma[\mathrm{pb}]$ & Eficiencia & $L\left[\mathrm{fb}^{-1}\right]$ \\
\hline$\gamma+$ jets $\left(p_{\mathrm{T}}^{\gamma}>70 \mathrm{GeV}\right)$ & SHERPA & 2153.0 & 1.0 & 1.160 \\
$\gamma+$ jets $\left(p_{\mathrm{T}}^{\gamma}>140 \mathrm{GeV}\right)$ & SHERPA & 137.85 & 1.0 & 10.881 \\
$\gamma+$ jets $\left(p_{\mathrm{T}}^{\gamma}>280 \mathrm{GeV}\right)$ & SHERPA & 5.963 & 1.0 & 167.657 \\
$\gamma+$ jets $\left(p_{\mathrm{T}}^{\gamma}>500 \mathrm{GeV}\right)$ & SHERPA & 0.276 & 1.0 & 3617.291 \\
$\gamma+$ jets $\left(p_{\mathrm{T}}^{\gamma}>800 \mathrm{GeV}\right)$ & SHERPA & 0.0133 & 1.0 & 7492.807 \\
$\gamma+$ jets $\left(p_{\mathrm{T}}^{\gamma}>1000 \mathrm{GeV}\right)$ & SHERPA & 0.00238 & 1.0 & 41980.269 \\
\hline$\gamma+$ jets $\left(p_{\mathrm{T}}^{\gamma}>70 \mathrm{GeV}\right)$ & PYTHIA 8 & 3425000 & 0.00057 & 1535.4 \\
$\gamma+$ jets $\left(p_{\mathrm{T}}^{\gamma}>140 \mathrm{GeV}\right)$ & PYTHIA8 & 122170 & 0.00097 & 8449.2 \\
$\gamma+$ jets $\left(p_{\mathrm{T}}^{\gamma}>280 \mathrm{GeV}\right)$ & PYTHIA 8 & 3348.7 & 0.00145 & 206559.7 \\
$\gamma+$ jets $\left(p_{\mathrm{T}}^{\gamma}>500 \mathrm{GeV}\right)$ & PYTHIA8 & 115.63 & 0.0018 & 4789097.0 \\
\hline Multijet $\left(p_{\mathrm{T}}^{\text {jet }} \in[20,80] \mathrm{GeV}\right)$ & PYTHIA & $7.285 \cdot 10^{10}$ & 0.000129 & 0.00016 \\
Multijet $\left(p_{\mathrm{T}}^{\text {jet }} \in[80,200] \mathrm{GeV}\right)$ & PYTHIA & $2.634 \cdot 10^{7}$ & 0.003894 & 0.0142 \\
Multijet $\left(p_{\mathrm{T}}^{\text {jet }} \in[200,500] \mathrm{GeV}\right)$ & PYTHIA & $5.442 \cdot 10^{5}$ & 0.001219 & 2.26 \\
Multijet $\left(p_{\mathrm{T}}^{\text {jet }} \in[500,1000] \mathrm{GeV}\right)$ & PYTHIA & 0.006445 & 0.000708 & 328 \\
Multijet $\left(p_{\mathrm{T}}^{\text {jet }} \in[1000,1500] \mathrm{GeV}\right)$ & PYTHIA & 39.74 & 0.002152 & 17400 \\
Multijet $\left(p_{\mathrm{T}}^{\text {jet }} \in[1500,2000] \mathrm{GeV}\right)$ & PYTHIA & 0.4161 & 0.004684 & $7.68 \cdot 10^{5}$ \\
Multijet $\left(p_{\mathrm{T}}^{\text {jet }}>2000 \mathrm{GeV}\right)$ & PYtHIA & 0.04064 & 0.0146 & $2.52 \cdot 10^{6}$ \\
\hline
\end{tabular}

Tabla 6.10: Muestras de Diboson. La sección eficaz a LO para cada modo de decaimiento, los factores $k$ (para la normalización NLO) y las eficiencias del filtro están detalladas, así como también la luminosidad integrada correspondiente a la estadística total de cada muestra.

\begin{tabular}{lcrrrr}
\hline Proceso & Generador & $\sigma[\mathrm{pb}]$ & Factor- $k$ & Eficiencia & $L\left[\mathrm{fb}^{-1}\right]$ \\
\hline$W W(\rightarrow \ell \ell \nu \nu)$ & SHERPA & 5.296 & 1.06 & 1.00 & 1400 \\
$Z Z(\rightarrow \ell \ell \nu \nu)$ & SHERPA & 0.494 & 1.05 & 1.00 & 1700 \\
$W Z \rightarrow \ell \ell \ell \nu)$ & SHERPA & 9.745 & 1.05 & 1.00 & 260 \\
$W Z \rightarrow \ell \nu \nu \nu)$ & SHERPA & 1.406 & 1.05 & 1.00 & 270 \\
$Z W(\rightarrow e e q q)$ & SHERPA & 1.465 & 1.05 & 1.00 & 110 \\
$Z Z(\rightarrow e \ell q q)$ & SHERPA & 0.247 & 1.00 & 1.00 & 120 \\
$Z W(\rightarrow \mu \mu q q)$ & SHERPA & 1.463 & 1.05 & 1.00 & 110 \\
$Z Z(\rightarrow \mu \mu q q)$ & SHERPA & 0.248 & 1.00 & 1.00 & 120 \\
$Z W(\rightarrow \tau \tau q q)$ & SHERPA & 1.452 & 1.05 & 1.00 & 120 \\
$Z Z(\rightarrow \tau \tau q q)$ & SHERPA & 0.242 & 1.00 & 1.00 & 120 \\
$Z W(\rightarrow \nu \nu q q)$ & SHERPA & 2.697 & 1.05 & 1.00 & 64 \\
$Z Z(\rightarrow \nu \nu q q)$ & SHERPA & 1.744 & 1.00 & 1.00 & 69 \\
$W W(\rightarrow e \nu q q)$ & SHERPA & 7.285 & 1.06 & 1.00 & 100 \\
$W Z(\rightarrow e \nu q q)$ & SHERPA & 1.904 & 1.05 & 1.00 & 110 \\
$W W(\rightarrow \mu \nu q q)$ & SHERPA & 7.297 & 1.06 & 1.00 & 100 \\
$W Z(\rightarrow \mu \nu q q)$ & SHERPA & 1.906 & 1.05 & 1.00 & 100 \\
$W W(\rightarrow \tau \nu q q)$ & SHERPA & 7.274 & 1.06 & 1.00 & 100 \\
$W Z(\rightarrow \tau \nu q q)$ & SHERPA & 1.915 & 1.05 & 1.00 & 100 \\
\hline
\end{tabular}




\section{7 \\ SELECCIÓN DE EVENTOS: DEFINICIÓN DE LAS REGIONES DE SENAL}

Como se discutió en la sección 5.2, las regiones de señal son un componente clave del análisis dado que son aquellas en las que se espera la presencia de eventos de nueva física. En este capítulo se describe la selección de eventos que define las regiones de señal del análisis de esta tesis. Esta selección fue optimizada en base a la significancia esperada de descubrimiento utilizando las muestras MC de señal y fondos del SM.

\subsection{Criterios de calidad sobre los datos}

Para este análisis se ha utilizado una GRL que, además de aplicar requerimientos generales sobre la operación del experimento y la estabilidad del haz en el acelerador, contiene aquellos eventos donde todos los subdetectores se encontraban funcionando en su plena capacidad.

Adicionalmente se eliminan los eventos que contengan algún tipo de problema, como ruido en el calorímetro, o celdas no operativas, que pueda resultar en energía faltante instrumental.

\section{2. $\quad$ Trigger}

Los datos fueron recolectados utilizando un trigger de un fotón (g120_loose), que selecciona eventos con al menos un fotón loose con momento transverso mayor a $120 \mathrm{GeV}$.

La eficiencia de este trigger fue calculada utilizando el método bootstrap siguiendo las prescripciones descriptas en $[146,147]$. Cuando se calcula la eficiencia del trigger utilizando el método bootstrap, se factoriza la eficiencia como el producto de la eficiencia del EF respecto a la eficiencia del L1 por la eficiencia de la selección de L1. La eficiencia de g120_loose respecto a fotones aislados con $p_{\mathrm{T}}>125 \mathrm{GeV}$ resulta en $100_{-1.41}^{+0}$ (stat.) ${ }_{-0.7}^{+0}$ (sist.) $\%$.

En la Figura 7.1 se puede ver el resultado como función del $p_{\mathrm{T}}$ del fotón. A pesar de la escasa estadística, es claro que la eficiencia alcanza una meseta, en la cual la eficiencia es máxima, antes de $125 \mathrm{GeV}$. La incerteza total tiene en cuenta la limitada estadística de la muestra de datos y también la incerteza en la corrección debido a la pureza de los fotones. El desempeño del trigger se mantuvo estable durante todo el período de la toma de datos considerado. 


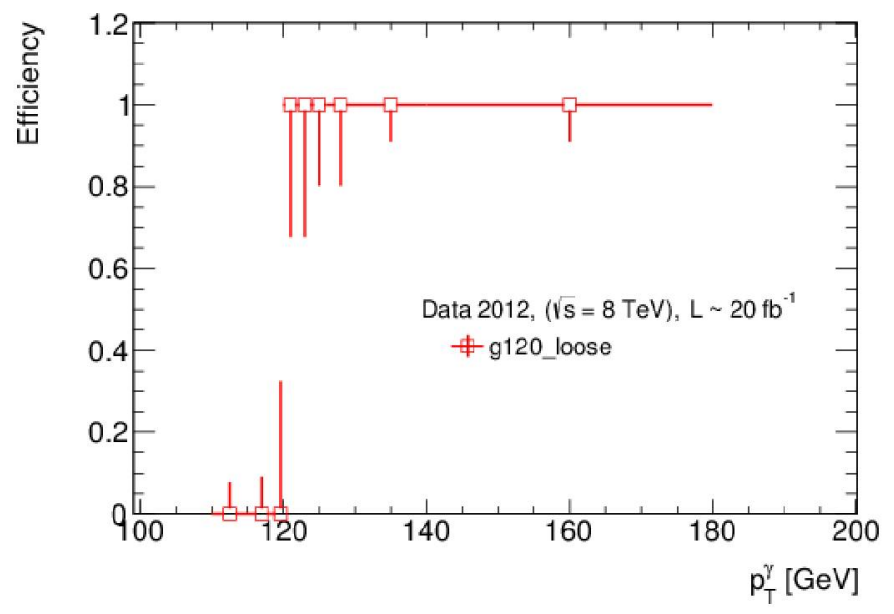

Figura 7.1: Eficiencia del trigger g120_loose como función del $p_{\mathrm{T}}$ del fotón.

\subsection{Preselección}

\subsubsection{Vértice primario}

Como se describe en la sección 3.1 los candidatos a vértices de la interacción $p p$ son reconstruidos en cada evento utilizando las trazas en el detector interno. El vértice primario, correspondiente a la interacción dura de dispersión, es el candidato con la mayor suma de $p_{\mathrm{T}}^{2}$ para las trazas asociadas. Solo se consideran los eventos donde el vértice primario tiene al menos cinco trazas cargadas.

\subsubsection{Objetos}

En primero lugar se realiza una preselección de los objetos que se consideran en cada evento como se detalla a continuación y se resume en la Tabla 7.1. Se consideran fotones todos aquellos candidatos a fotón que pasen el criterio de identificación tight, que tengan $p_{\mathrm{T}}>75 \mathrm{GeV}$ y $|\eta|<2.37$. Se consideran electrones los candidatos que pasan el criterio de identificación medium, y que tengan un $p_{\mathrm{T}}>10 \mathrm{GeV}$ y $|\eta|<2.47$, y muones a aquellos que pasan un criterio de identificación loose con $p_{\mathrm{T}}>6 \mathrm{GeV}$ y $|\eta|<2.5$. Para el caso de jets, se consideran los que tienen $p_{\mathrm{T}}>20 \mathrm{GeV}$ y $|\eta|<2.8$.

Tabla 7.1: Preselección de objetos. Criterio de identificación (ID) y cortes de aceptancia $\left(p_{\mathrm{T}}, \eta\right)$ considerados para cada tipo de partícula. Además del límite superior en $|\eta|$, no se consideran en ningún caso los objetos que se encuentren en la región entre la zona del barrel y las end-caps del detector, es decir, con $1.37<|\eta|<1.52$.

\begin{tabular}{lccc}
\hline & ID & $p_{\mathrm{T}}$ & $|\eta|$ \\
\hline Fotones & tight & $>75 \mathrm{GeV}$ & $<2.37$ \\
Electrones & medium & $>10 \mathrm{GeV}$ & $<2.47$ \\
Muones & loose & $>6 \mathrm{GeV}$ & $<2.5$ \\
Jets & - & $>20 \mathrm{GeV}$ & $<2.8$ \\
\hline
\end{tabular}




\subsubsection{Eliminación de objetos superpuestos}

De acuerdo a las definiciones de objetos presentadas más arriba, un objeto puede estar en más de una categoría, contándose dos veces. Por este motivo se realiza un procedimiento para remover este solapamiento, el cual se aplica sobre los objetos preseleccionados antes de aplicar los criterios de aislamiento. El procedimiento para remover estas ambigüedades entre los objetos es el que se detalla a continuación en ese orden:

- Si un fotón o electrón se encuentran dentro de $\Delta R<0.01$, el objeto es considerado como un electrón, removiéndose el fotón correspondiente. Esta elección reduce la tasa de electrones mal reconstruidos como fotones.

- Los jets que estén cerca $(\Delta R<0.2)$ de un electrón o fotón preseleccionado se remueven.

- Fotones y electrones preseleccionados son removidos si su distancia al jet más cercano es $0.2<\Delta R<0.4$.

- Muones preseleccionados son removidos si su distancia al jet más cercano es $\Delta R<0.4$.

Por último, para asegurar el correcto cálculo de la energía faltante, y reducir los eventos con energía faltante instrumental, los eventos que satisfacen alguna de las condiciones siguientes son descartados.

- Si el evento (después de de la preselección de objetos y la eliminación de los objetos superpuestos) contiene al menos un jet que falla los cortes de limpieza de jets que se definen en sección 3.4.

- Eventos con muones cósmicos: cuando el evento tiene al menos un muon con $\left|z_{0}\right|>1 \mathrm{~mm} o$ $\left|d_{0}\right|>0.2 \mathrm{~mm}$, donde estos valores son calculados con respecto al vértice primario.

Después de esta limpieza de eventos, con los objetos definidos en las secciones anteriores y después de haber eliminado los objetos duplicados, se calcula la energía faltante transversa como se explica en sección 3.5 .

\subsection{Optimización de la selección de las regiones de señal}

Como primer paso se seleccionan eventos que contengan el estado final buscado: un fotón, al menos un jet y energía faltante, y, a partir de esa selección inicial, se eligen observables que permitan separar la señal del fondo y se optimizan los cortes en estos observables.

Del estudio realizado en la sección 6.2.1, resultan evidentes las diferencias cinemáticas y topológicas de los eventos en las distintas regiones del espacio de parámetros $\left(m_{\widetilde{g}}, m_{\widetilde{\chi}_{1}^{0}}\right)$ del modelo de SUSY. ES por esta razón que resulta conveniente tener más de una región de señal, cada una destinada a una zona particular del espacio de parámetros.

Por tal motivo, se dividió el análisis en dos regiones de señal, a las que se llamó $\mathrm{SR}_{\mathrm{L}} \mathrm{y} \mathrm{SR}_{\mathrm{H}}$. La primera de ellas, $\mathrm{SR}_{\mathrm{L}}$, se focaliza en la zona en la que los gluinos decaen en cascada hasta neutralinos NLSP de baja y moderada masa. Los eventos de señal en esta región se caracterizan por una gran multiplicidad de jets y actividad hadrónica, debido a que involucra cadenas de decaimiento largas de gluinos pesados a través de la producción de charginos y quarks. Cuanto más baja la masa del $\widetilde{\chi}_{1}^{0}$, más baja sera la energía del fotón y menor es la cantidad de energía faltante. La segunda región de señal, $\mathrm{SR}_{\mathrm{H}}$, tiene como motivación aquellos escenarios donde la masa del gluino y del neutralino más liviano están cerca una de la otra. La cadena de decaimiento es por lo tanto mucho más corta en este caso, con una menor multiplicidad de jets $\mathrm{y}$, debido al neutralino pesado, fotones de alto $p_{\mathrm{T}} \mathrm{y}$ gran cantidad de energía faltante en el estado final. 
En la Figura 7.2 puede verse esquemáticamente a que región del espacio de parámetros de la señal $\left(m_{\tilde{g}}, m_{\widetilde{\chi}_{1}^{0}}\right)$ está destinada cada SR.

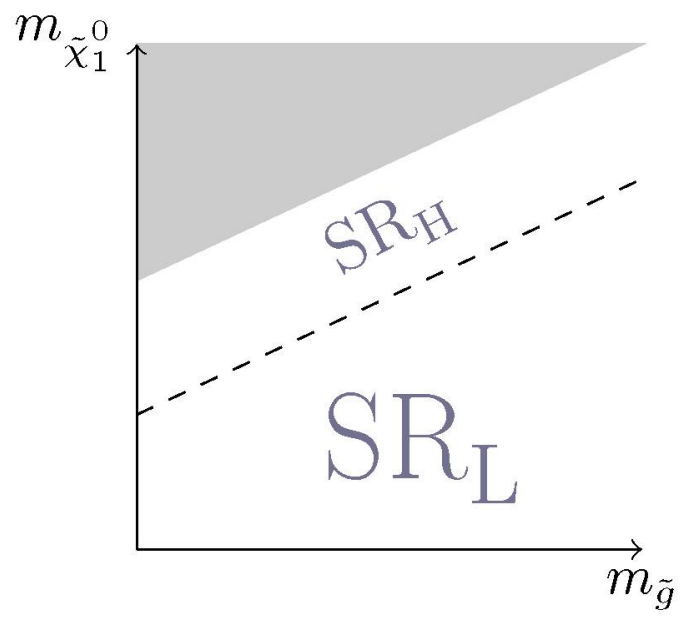

Figura 7.2: Esquema de la región del espacio de parámetros del modelo de señal en el plano $\left(m_{\tilde{g}}, m_{\widetilde{\chi}_{1}^{0}}\right)$ y a que zona esta dirigido el diseño de las dos regiones de señal.

Para la optimización de las SR, se utilizaron varios puntos de señal en cada región, representativos de la topología y el espacio de fase en cada caso:

- $\mathrm{SR}_{\mathrm{L}}:(1150,200) \mathrm{y}(1150,450)$

- $\mathrm{SR}_{\mathrm{H}}:(1150,850)$ y $(1150,1050)$

donde cada punto queda determinado por sus valores $\left(M_{3}, \mu\right)$.

Como fondo se utilizaron las muestras MC, y se consideró una incerteza en la estimación del fondo de $25 \%$. Todas las muestras MC fueron normalizadas a una luminosidad total integrada de $20.3 \mathrm{fb}^{-1}$. La significancia esperada se calculó utilizando la ecuación (4.34), y para evitar una selección muy restringida, se requiere al menos un evento de fondo en cada SR. Además de evaluar la significancia esperada para distintos cortes también se tuvo en cuenta que la eficiencia de señal sea superior al $20 \%$.

Los cortes fueron optimizados en el orden de poder discriminatorio, uno a la vez, y aplicados en el orden descripto a continuación, monitoreando la significancia para distintos cortes en las variables discriminatorias.

Como ya se ha mencionado, el estado final buscado en este análisis consiste en un único fotón, jets y energía faltante. Por lo que la definición de las SR comienza optimizando los cortes en las variables cinemáticas del fotón, los jets y el corte en energía faltante.

\subsubsection{Fotón}

El primer paso para definir la SR consistió en seleccionar eventos con un único fotón de los previamente seleccionados con $p_{\mathrm{T}}$ mayor a $125 \mathrm{GeV}$. Este valor fue elegido para asegurar la máxima eficiencia del trigger.

Los eventos con un segundo fotón con $p_{\mathrm{T}}>75 \mathrm{GeV}$ fueron vetados. El corte en $p_{\mathrm{T}}$ de $75 \mathrm{GeV}$ fue elegido para que las SR sean ortogonales a las SR del análisis de ATLAS que busca dos fotones [27] garantizando la posible combinación estadística entre ambos. Este análisis fue diseñado para 
buscar SUSY en escenarios GGM, pero particularmente en el caso de que el neutralino NLSP sea mayoritariamente bino, y el decaimiento a fotones sea dominante.

Como se menciona en la sección 3.2.2, para seleccionar los fotones se aplica un corte en la energía transversa de aislamiento $\left(E_{\mathrm{T}}^{\text {iso }}\right)$. Específicamente, para el presente análisis se estudiaron distintos criterios de aislamiento, variando el tamaño del cono $(R)$ en el cual se calcula esta energía.

En la Figura 7.3 se muestran las distribuciones de la energía de aislamiento para varios valores de $R$ para las muestras de señal en las dos SR. La SR con mayor diferencia de masa entre gluino y neutralino parece tener distribuciones más anchas, un efecto que desaparece para tamaños de cono más chicos. Como se ve en la Figura 7.4 (arriba), la mayor eficiencia de selección se obtiene también para el menor tamaño de cono, $R=0.2$. Para un corte de $5 \mathrm{GeV}$, se obtiene una eficiencia alta (85-90\%) a lo largo de toda la grid. Luego de aplicar una preselección en $E_{\mathrm{T}}^{\text {miss }}$, los fotones no aislados provenientes de fondos dominados por procesos QCD son muy suprimidos, reduciendo el impacto del corte de aislamiento en la significancia.

Incluso luego de que la corrección es aplicada para remover la filtración de energía del fotón dentro de su propio cono de aislamiento, una dependencia remanente con el $p_{\mathrm{T}}$ del fotón es observada para todos los tamaños de cono considerados, como se muestra en Figura 7.3 (abajo). El tratamiento de este efecto es discutido en la secciones 8.4.2 y 9.2.1.
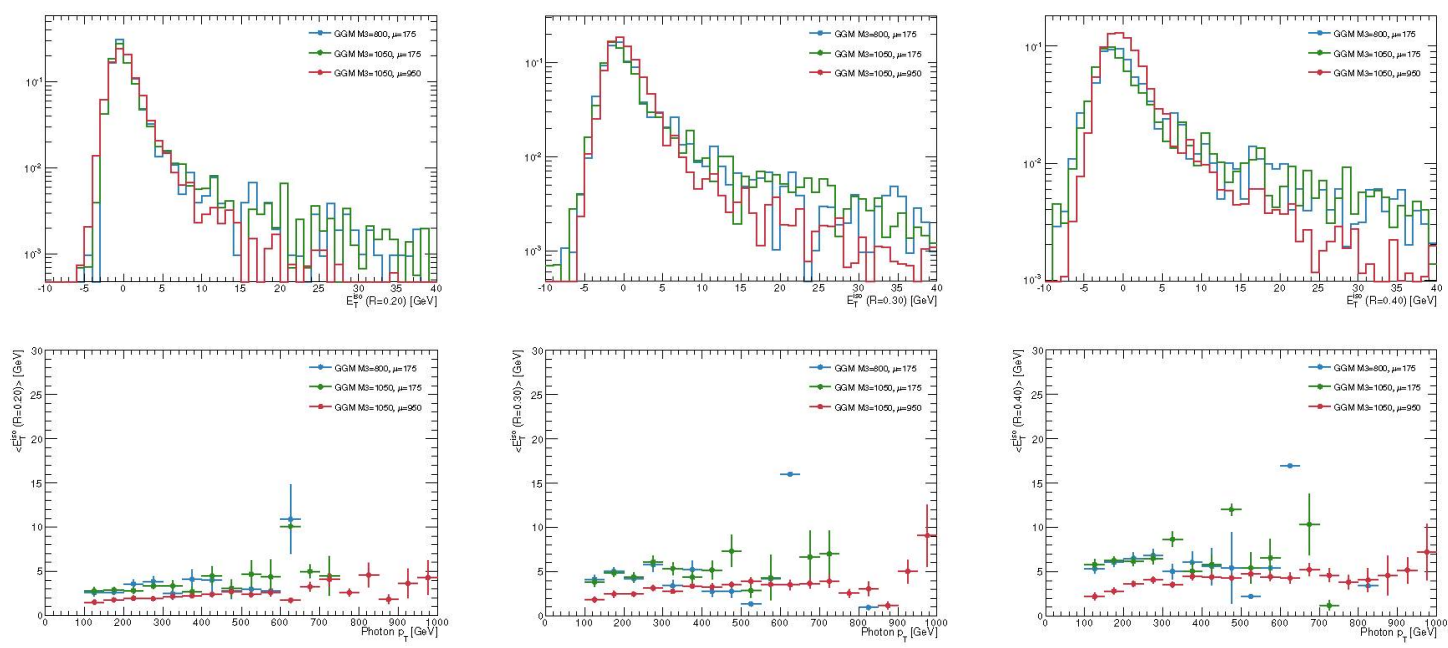

Figura 7.3: Distribución de $E_{\mathrm{T}}^{\text {iso }}$ (arriba) y su valor medio como función del $p_{\mathrm{T}}$ del fotón (abajo) para distintos tamaños de conos: $R=0.2, R=0.3 \mathrm{y} R=0.4$. Una preselección de $p_{\mathrm{T}}>125 \mathrm{GeV}$ y $E_{\mathrm{T}}^{\text {miss }}>150 \mathrm{GeV}$ fue aplicada, para suprimir gran parte del fondo de fotones directos.

\subsubsection{Leptones}

Además del veto a los eventos con un segundo fotón, también se remueven los eventos que contienen leptones $(e \circ \mu)$ con el objetivo de que las SR no tengan un solapamiento con las SR del análisis que busca estados finales con un fotón, un leptón y energía faltante [28].

\subsubsection{Energía faltante}

La principal característica de los eventos de SUSY donde se conserva la paridad-R es la presencia de una gran cantidad de energía faltante debido a las LSP estables que escapan la detección, en este 

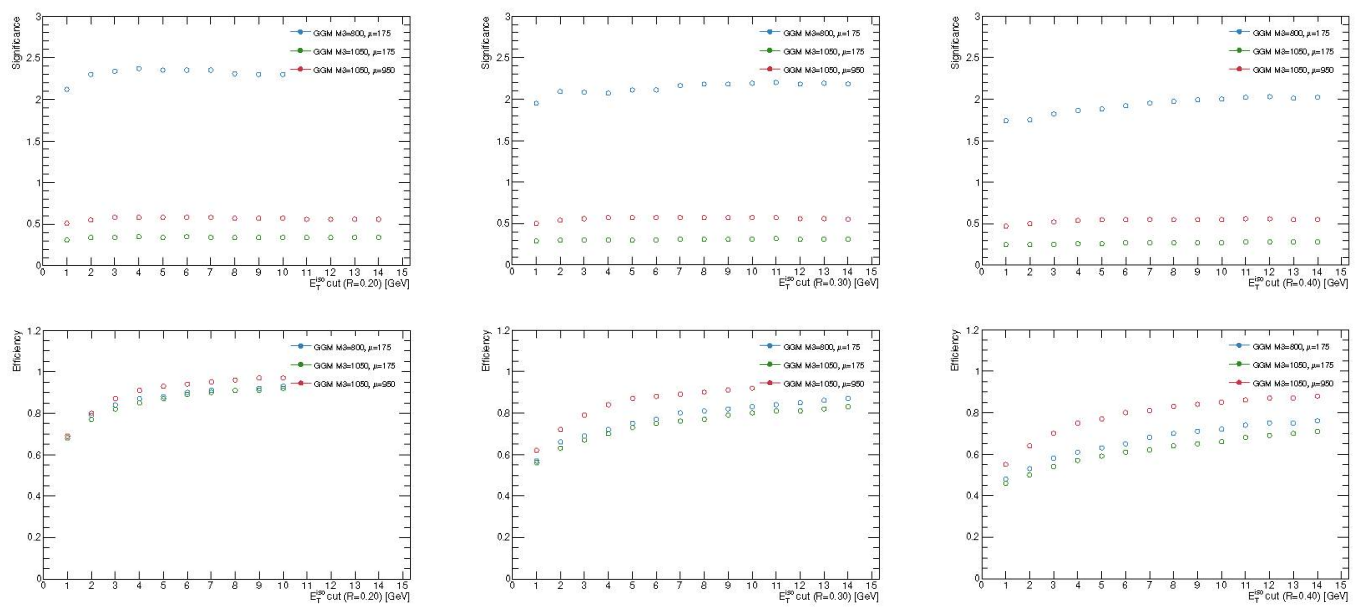

Figura 7.4: Significancia esperada (arriba) y eficiencia (abajo) como función del corte en la energía de aislamiento, después del corte en $p_{\mathrm{T}}$ y $E_{\mathrm{T}}^{\mathrm{miss}}$.

caso debido a los dos gravitinos en el estado final. Un corte en esta variable reduce enormemente la contaminación de procesos QCD y también de otros procesos del Modelo Estándar. La Figura 7.5 muestra la distribución de $E_{\mathrm{T}}^{\mathrm{miss}}$ en eventos con un fotón aislado con alto $p_{\mathrm{T}}$ (como se definió en la sección anterior), para dos puntos de señal en cada SR, junto al fondo esperado de simulaciones MC.

Un corte en $E_{\mathrm{T}}^{\text {miss }}$ relativamente bajo $(>200 \mathrm{GeV})$ es capaz de separar la señal y el fondo significativamente en $\mathrm{SR}_{\mathrm{L}}$, mientras que para $\mathrm{SR}_{\mathrm{H}}$ es posible aplicar un corte más alto (> $\left.300 \mathrm{GeV}\right)$.
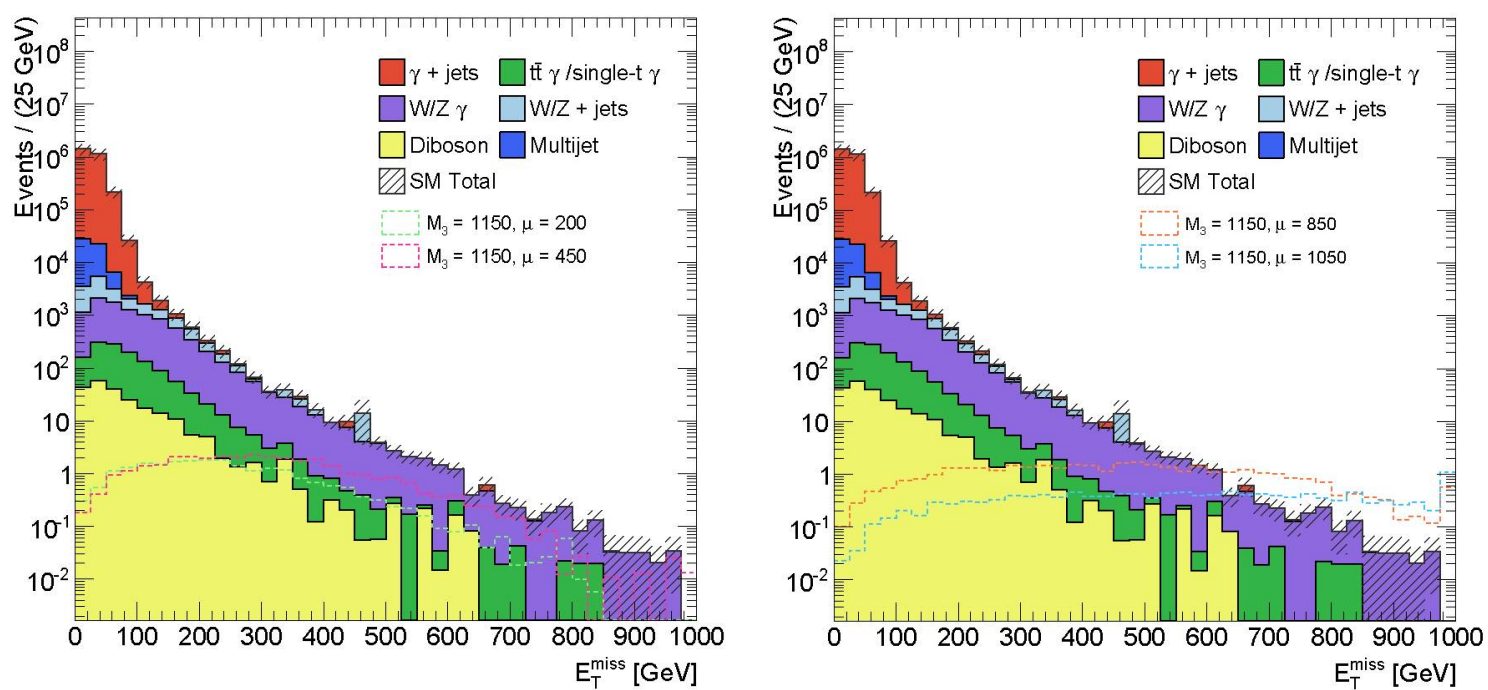

Figura 7.5: Energía faltante transversa luego de aplicar el corte en el $p_{\mathrm{T}}$ del fotón y en la energía de aislamiento para el fondo y los puntos de señal de $\mathrm{SR}_{\mathrm{L}}$ (izquierda) y $\mathrm{SR}_{\mathrm{H}}$ (derecha), para una luminosidad integrada de $20.3 \mathrm{fb}^{-1}$.

\subsubsection{Multiplicidad y $p_{\mathrm{T}}$ de los jets}

En la Figura 7.6 se muestra el valor medio del número de jets seleccionados como función del número de vértices en el evento, para distintos cortes en el $p_{\mathrm{T}}$ de los jets. Notar que el número de 
vértices en un evento está relacionado con el pile-up. A medida que aumenta el corte en $p_{\mathrm{T}}$ el número de jets se hace más estable con el pile-up, y por lo tanto en este análisis se consideran los jets con $p_{\mathrm{T}}>40 \mathrm{GeV}$

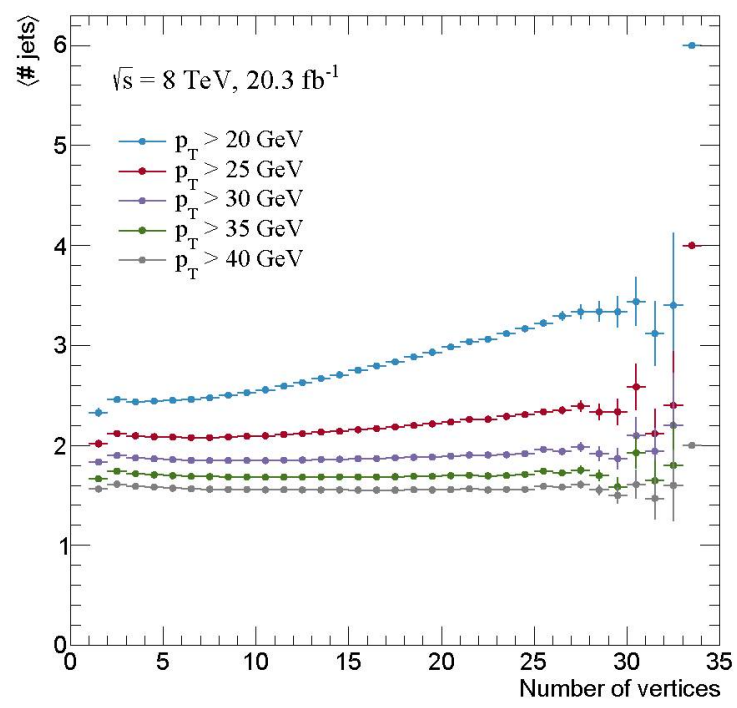

Figura 7.6: Valor medio del número de jets vs. el número de vértices primarios observados en datos para distintas selecciones de $p_{\mathrm{T}}^{\text {jet }}$.

La Figura 7.7 muestra la multiplicidad de jets $\left(N_{\text {jets }}\right)$ para $p_{\mathrm{T}}^{\text {jet }}>40 \mathrm{GeV}$, después de los cortes optimizados en el fotón y la $E_{\mathrm{T}}^{\text {miss }}$ descriptos anteriormente. Mientras que la señal tiene típicamente un gran número de jets, la mayoría de los fondos $\mathrm{W} / \mathrm{Z}+$ jets, diboson y QCD se acumulan en la zona de pocos jets. Al menos dos (cuatro) jets son requeridos para los eventos que pasan la selección de $\mathrm{SR}_{\mathrm{L}}\left(\mathrm{SR}_{\mathrm{H}}\right)$. Una mayor multiplicidad es efectivamente esperada para $\mathrm{SR}_{\mathrm{L}}$ debido a que su objetivo es la región de gluinos pesados donde el decaimiento dominante es el de producción de charginos por un decaimiento de tres cuerpos.

En la $\mathrm{SR}_{\mathrm{L}}$ es posible obtener una discriminación requiriendo además que los dos jets más energéticos tengan $p_{\mathrm{T}}>100 \mathrm{GeV}$.

\subsubsection{Separación angular entre jets y $E_{\mathrm{T}}^{\mathrm{miss}}$}

Como ya ha sido mencionado, en los eventos de señal, la energía faltante es producida por los dos gravitinos que escapan la detección. Se espera por lo tanto que la dirección de $E_{\mathrm{T}}^{\mathrm{miss}}$ sea aleatoria, sin estar correlacionada con ninguno de los demás objetos del evento. Por otro lado, si la $E_{\mathrm{T}}^{\text {miss }}$ es originada a partir de efectos instrumentales o de neutrinos energéticos en $b$-jets o $c$-jets, la dirección de $E_{\mathrm{T}}^{\text {miss }}$ estará correlacionada con uno de los jets mal reconstruidos. La Figura 7.8 muestra el mínimo $\Delta \phi$ entre la dirección de $E_{\mathrm{T}}^{\text {miss }}$ y la de los dos jets más energéticos.

\subsubsection{Separación angular entre jets y fotón}

Para regiones del espacio de parámetros del modelo de señal donde los gluinos y los neutralinos tienen alta masa $\left(\mathrm{SR}_{\mathrm{H}}\right)$ un corte en la separación entre el fotón y los jets ayuda a reducir el fondo, principalmente aquel proveniente de dijets y fotones directos, donde un fotón (real o falso) tiende a ser 

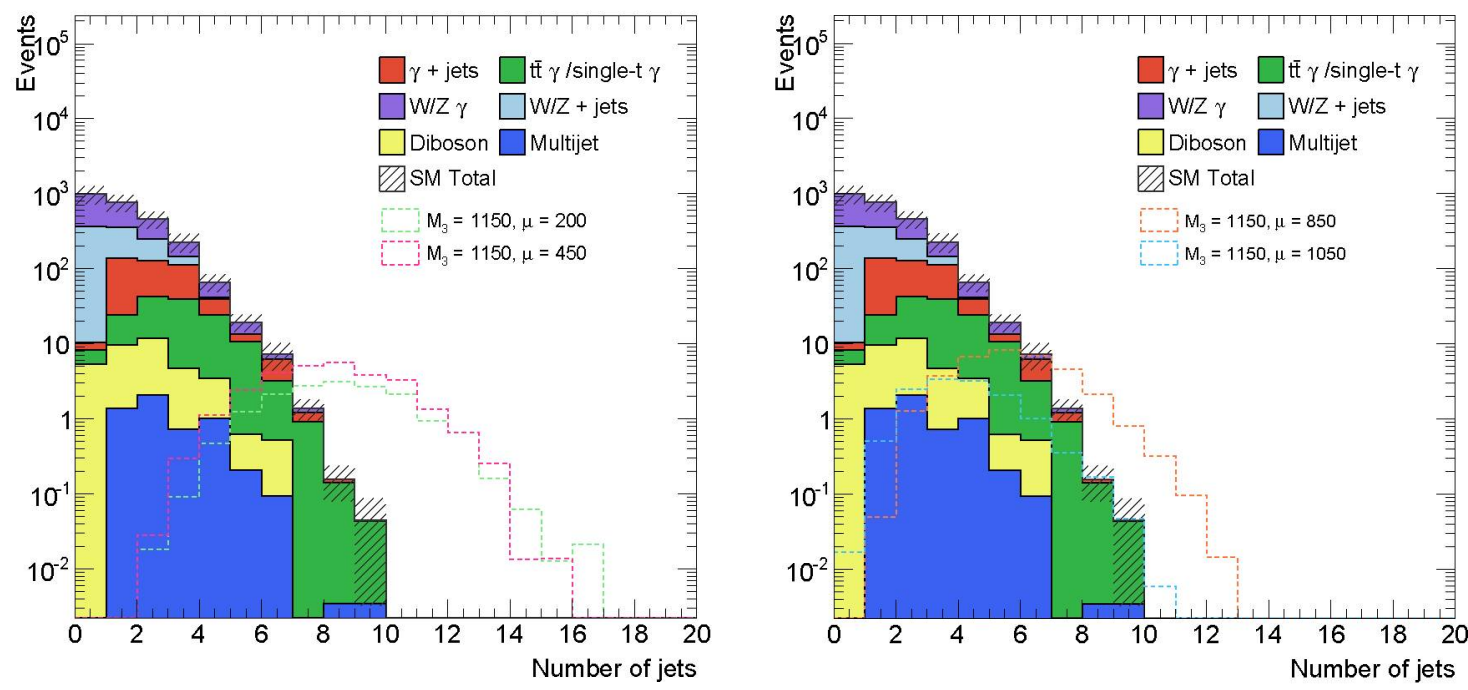

Figura 7.7: Distribuciones del número de jets con $p_{\mathrm{T}}>40 \mathrm{GeV}$ en $\mathrm{SR}_{\mathrm{L}}$ (izquierda) y $\mathrm{SR}_{\mathrm{H}}$ (derecha), después de la selección en el $p_{\mathrm{T}}$ del fotón y $E_{\mathrm{T}}^{\text {miss }}>150 \mathrm{GeV}$, para una luminosidad integrada de 20.3 $\mathrm{fb}^{-1}$.
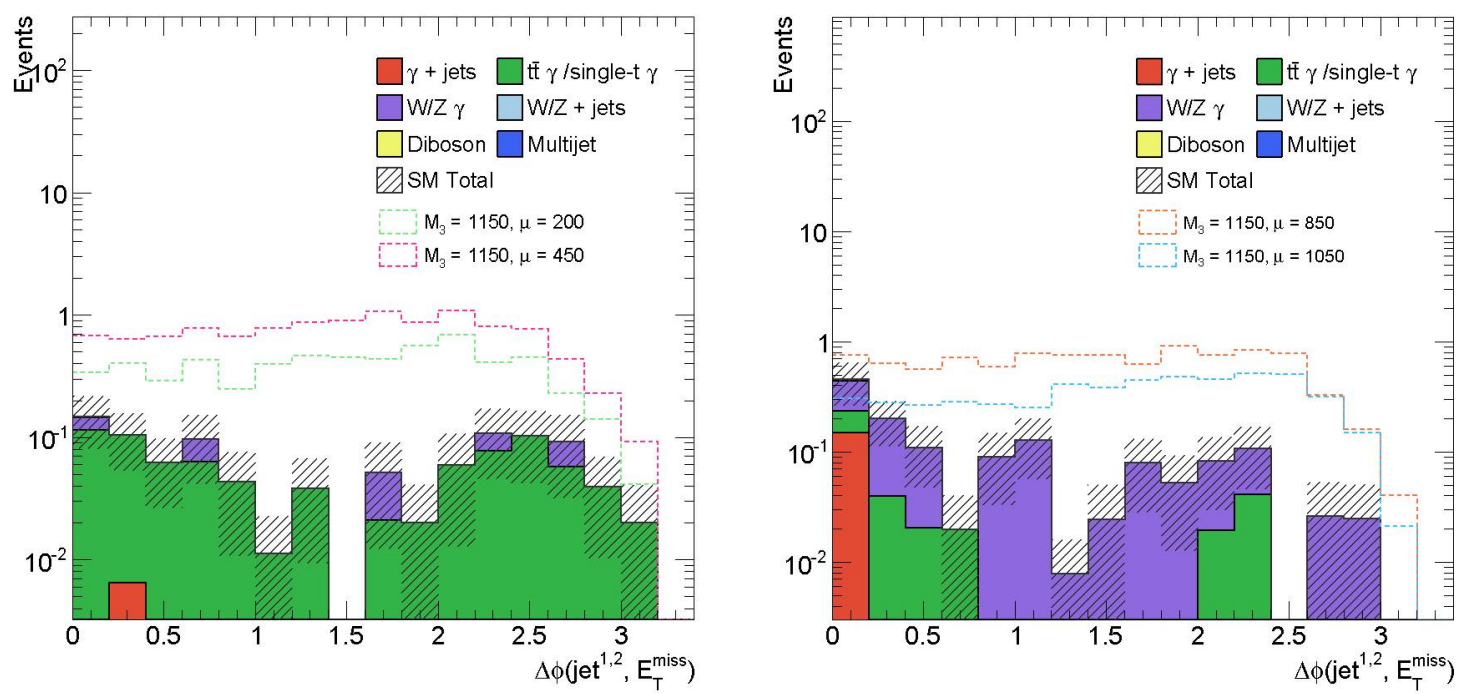

Figura 7.8: Separación angular $\Delta \phi\left(\right.$ jet, $\left.\mathrm{E}_{\mathrm{T}}^{\text {miss }}\right)$ para el fondo y los puntos de señal para $\mathrm{SR}_{\mathrm{L}}$ (izquierda) y $\mathrm{SR}_{\mathrm{H}}$ (derecha), para una luminosidad integrada de $20.3 \mathrm{fb}^{-1}$.

producido en la dirección opuesta al jet más energético. La distribución para la $\mathrm{SR}_{\mathrm{H}}$ se puede ver en Figura 7.9.

\subsubsection{Energía total transversa $\left(H_{\mathrm{T}}\right)$}

Dada la gran masa de los gluinos producidos en las colisiones, en el espacio de parámetros explorado en este análisis, se espera que la energía transversa visible total sea alta. Por eso, el observable $H_{\mathrm{T}}$ definido como la suma escalar del momento transverso de todos los objetos observados en el estado final es mucho mayor para eventos de señal que para los eventos de fondo. Después del veto a los eventos con leptones, $H_{\mathrm{T}}$ se define como: 


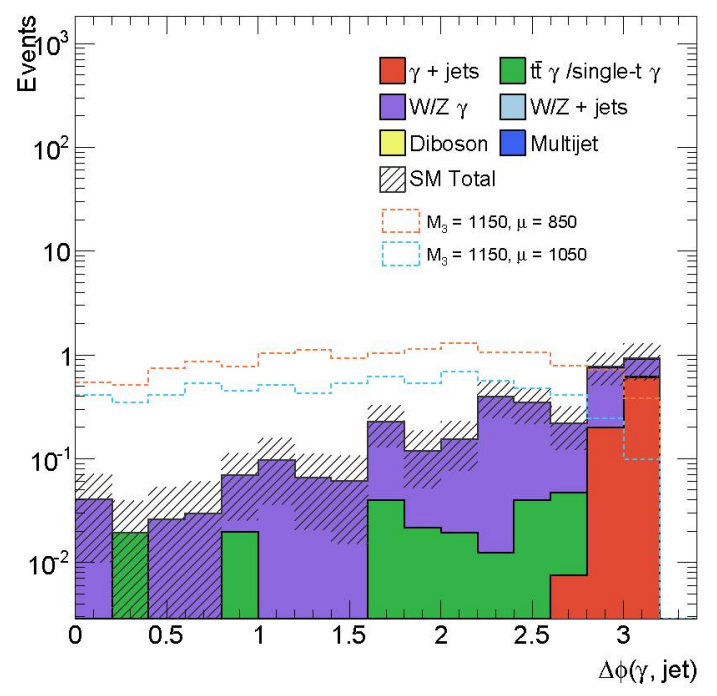

Figura 7.9: Distribución de $\Delta \phi(\mathrm{jet}, \gamma)$ para $\mathrm{SR}_{\mathrm{H}}$, después de aplicar la selección completa, excepto este corte, para una luminosidad integrada de $20.3 \mathrm{fb}^{-1}$.

$$
H_{\mathrm{T}} \equiv\left|p_{\mathrm{T}}^{\gamma}\right|+\sum_{\text {jets }}\left|p_{\mathrm{T}}^{\text {jet }}\right|
$$

La Figura 7.10 muestra las distribuciones de $H_{\mathrm{T}}$ para las dos regiones de señal. Para $\mathrm{SR}_{\mathrm{L}}$, se espera incluso que $H_{\mathrm{T}}$ sea mayor, debido a la diferencia de masa entre el gluino y el neutralino NLSP. Sin embargo se encontró una variable más efectiva para la separación entre señal y fondo que se describe a continuación y por lo tanto no se aplica un corte en esta variable.

En $\mathrm{SR}_{\mathrm{H}}$ un corte en este observable $H_{\mathrm{T}}>800 \mathrm{GeV}$ ayuda a reducir el fondo restante. Dada la masa alta del neutralino, la mayor contribución de $H_{\mathrm{T}}$ proviene del fotón muy energético producido en el evento.
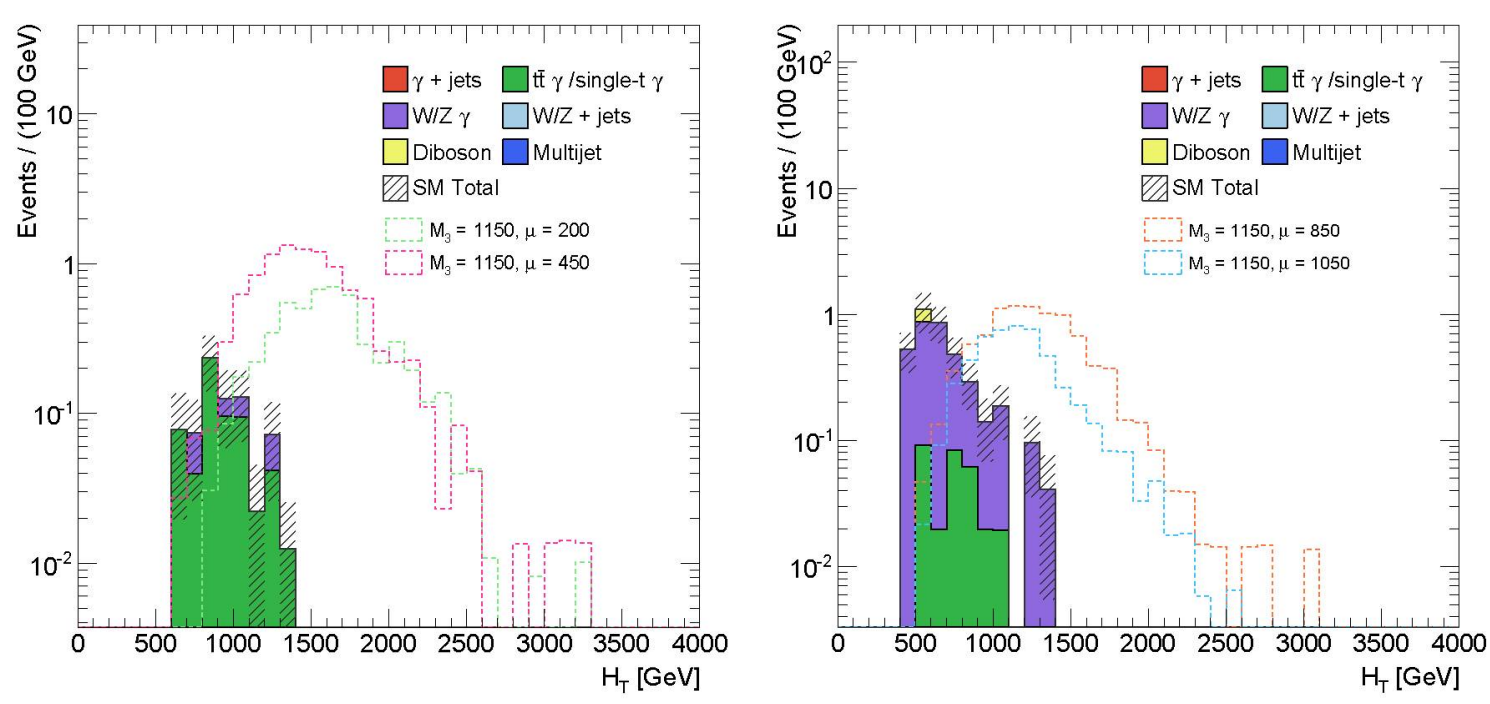

Figura 7.10: Distribución de la energía total transversa $\left(H_{\mathrm{T}}\right)$ para $\mathrm{SR}_{\mathrm{L}}$ (izquierda) y $\mathrm{SR}_{\mathrm{H}}$ (derecha) para una luminosidad total integrada de $20.3 \mathrm{fb}^{-1}$. Todos los cortes de la selección de la SR son aplicados exceptuando el corte en $H_{\mathrm{T}}$. 


\subsubsection{Variables de forma adicionales}

Varias variables de forma fueron exploradas para poder obtener una discriminación adicional entre señal y fondo. En especial se estudió el observable $R_{T}^{n}$ definido como

$$
R_{\mathrm{T}}^{n}=\frac{\sum_{i=1}^{n} p_{\mathrm{T}}^{\text {jet }_{i}}}{\sum p_{\mathrm{T}}^{\text {jet }}}
$$

es decir, el cociente entre la suma escalar de los $p_{\mathrm{T}}$ de $n$ jets y la suma escalar del $p_{\mathrm{T}}$ de todos los jets en el evento, donde $n$ es el mínimo número de jets requerido.

Es evidente de su definición que la distribución de esta variable depende de la multiplicidad y el momento transverso de los jets [148]. En el caso que $n \sim N_{\text {jets }}$ el denominador y el numerador son casi idénticos y el cociente es $\sim 1$, y es exactamente 1 cuando son iguales. Para los procesos de señal en los que $n$ es mucho menor al número de jets en el evento, la distribución será menor a 1.
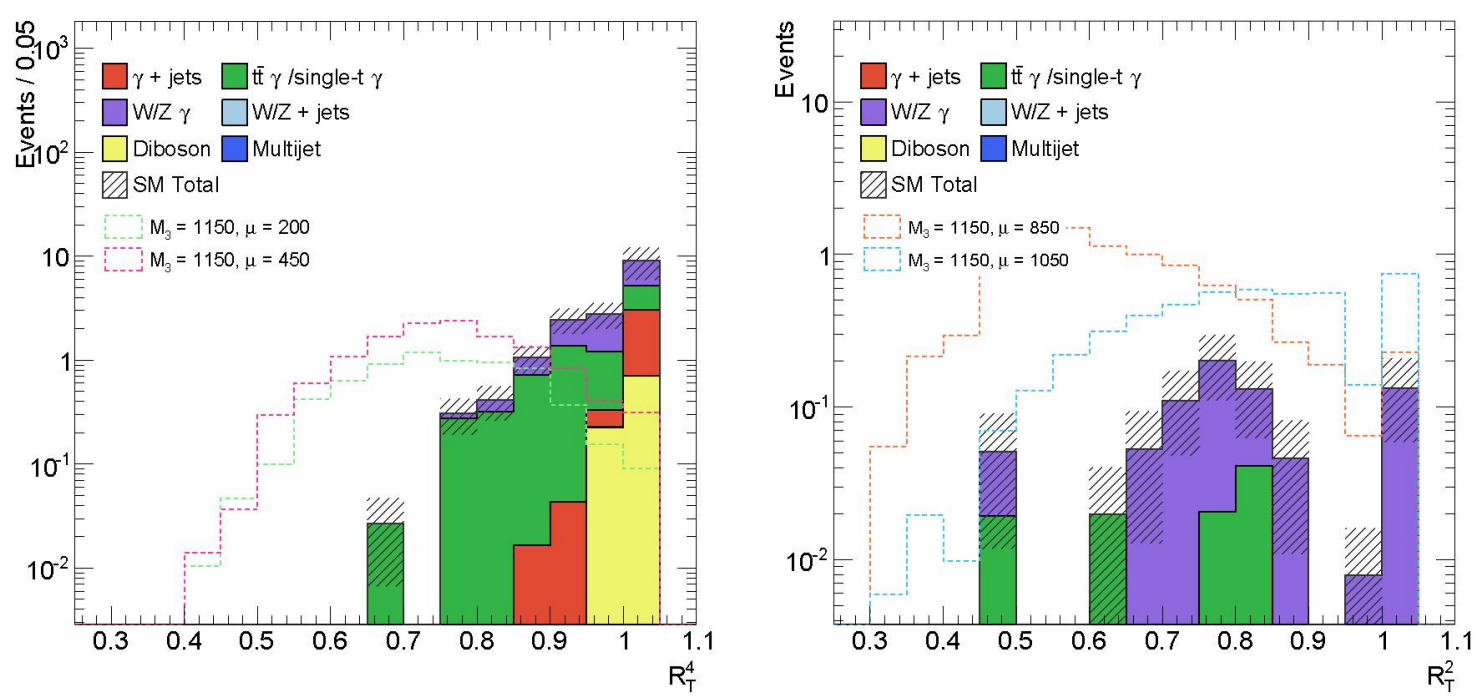

Figura 7.11: Distribución de $R_{\mathrm{T}}^{4}$ para $\mathrm{SR}_{\mathrm{L}}$ (izquierda) y $R_{\mathrm{T}}^{2}$ para $\mathrm{SR}_{\mathrm{H}}$ (derecha) para una luminosidad total integrada de $20.3 \mathrm{fb}^{-1}$. Todos los cortes de la selección de la SR son aplicados exceptuando el corte en $R_{\mathrm{T}}$.

La Figura 7.11 (izquierda) muestra la distribución de $R_{\mathrm{T}}^{2}$ para $\mathrm{SR}_{\mathrm{H}}$, después de la selección final excepto el corte en $R_{\mathrm{T}}^{2}$. Mientras que la Figura 7.11 muestra la distribución de $R_{\mathrm{T}}^{4}$ para $\mathrm{SR}_{\mathrm{L}}$. El bajo número de jets para $\mathrm{SR}_{\mathrm{H}}$, hace que la distribución de $R_{\mathrm{T}}^{2}$ para los eventos de señal sea similar a la del fondo QCD, y por lo tanto el observable no es útil para rechazar fondo manteniendo una aceptancia razonable. Para el caso de $\mathrm{SR}_{\mathrm{L}}$ un corte $R_{\mathrm{T}}^{4}<0.85$ permite reducir en una fracción considerable el fondo con una pérdida muy chica en la eficiencia de señal.

\subsection{Selección final de las regiones de señal}

Como resultado del proceso de optimización descripto en la sección anterior, se definen las dos regiones de señal:

$\mathbf{S R}_{\mathrm{L}}$ motivada por los eventos en los que gluinos decaen a neutralinos de baja/media masa. Estos eventos están caracterizados por una gran multiplicidad de jets energéticos, mientras que la 
energía del fotón y la energía faltante dependerá de la masa del neutralino, pero en general será relativamente baja.

$\mathbf{S R}_{\mathbf{H}}$ motivada por eventos en los que los gluinos producidos decaen en neutralinos con una masa similar a estos. Dichos eventos están caracterizados por un fotón de alto $p_{\mathrm{T}}$ y gran cantidad de $E_{\mathrm{T}}^{\text {miss }}$, con una menor multiplicidad de jets.

Cada región de señal queda definida entonces por los cortes de selección detallados en la Tabla 7.2.

Tabla 7.2: Conjunto de cortes en los observables que definen las dos regiones de señal, $\mathrm{SR}_{\mathrm{L}}$ y $\mathrm{SR}_{\mathrm{H}}$.

\begin{tabular}{lrr}
\hline & $\mathrm{SR}_{\mathrm{L}}$ & $\mathrm{SR}_{\mathrm{H}}$ \\
\hline$N_{\text {fotones }}$ & $=1$ & $=1$ \\
$p_{\mathrm{T}}^{\gamma}$ & $>125 \mathrm{GeV}$ & $>300 \mathrm{GeV}$ \\
$N_{\text {leptones }}$ & $=0$ & $=0$ \\
$N_{\text {jets }}$ & $\geq 4$ & $\geq 2$ \\
$p_{\mathrm{T}}^{\text {jet }}$ & $>100 \mathrm{GeV}$ & - \\
$p_{\mathrm{T}}^{\text {jet }}$ & $>100 \mathrm{GeV}$ & - \\
$\Delta \phi\left(\right.$ jet,$\left.E_{\mathrm{T}}^{\text {miss }}\right)$ & $>0.4$ & $>0.4$ \\
$E_{\mathrm{T}}^{\text {miss }}$ & $>200 \mathrm{GeV}$ & $>300 \mathrm{GeV}$ \\
$R_{\mathrm{T}}^{4}$ & 0.85 & - \\
$H_{\mathrm{T}}$ & - & $>800 \mathrm{GeV}$ \\
$\Delta \phi(\gamma$, jet $)$ & - & $<2.0$ \\
\hline
\end{tabular}

El número esperado de eventos de fondos del Modelo Estándar estimado a partir de de las muestras simuladas después de la selección final se muestra en la Tabla 7.3, para $\mathrm{SR}_{\mathrm{L}}$ y $\mathrm{SR}_{\mathrm{H}}$, respectivamente. También se presenta el número de eventos esperado de señal y la significancia esperada para los dos puntos de referencia en cada SR. Adicionalmente, en la Tabla 7.4 se puede ver el número de eventos de fondo esperado separando fotones reales de los eventos donde un jet o un electrón es identificado erróneamente como un fotón. Se puede ver que la contaminación dominante proviene de $V \gamma(V=W \circ Z)$ y $t \bar{t} \gamma$. La contaminación de $V \gamma$ en $\mathrm{SR}_{\mathrm{L}}$ es de 0.13 , de los cuales 0.10 corresponden a $W \gamma$ y 0.03 a $Z(\rightarrow \nu \nu) \gamma$. Para $\mathrm{SR}_{\mathrm{H}}$ el número de eventos de $V \gamma$ es de 0.66 , de los cuales 0.44 de $W \gamma \mathrm{y}$ 0.22 de $Z(\rightarrow \nu \nu) \gamma$. En la $\mathrm{SR}_{\mathrm{L}}$ también existe una contaminación de eventos de procesos $t \bar{t}$ en los que un electrón es identificado como fotón.

La significancia esperada de descubrimiento para los distintos puntos de la grid de señal en el plano $\left(m_{\widetilde{g}}, m_{\widetilde{\chi}_{1}^{0}}\right)$ se puede ver en la Figura 7.12 , junto con los contornos en los que la significancia es de $3 \sigma$ y $5 \sigma$.

Es importante notar que estos resultados son preliminares y obtenidos de simulaciones Monte Carlo. Los resultados finales son derivados utilizando los métodos de estimación de fondos descriptos en el capítulo 8, y luego del ajuste combinado, y son descriptos en el capítulo 9.

\subsection{Aceptancia y eficiencia}

La aceptancia se define como:

$$
A=\frac{N_{\text {fiducial }}}{N_{\text {total }}}
$$


Tabla 7.3: Número de eventos esperado para los fondos del Modelo Estándar y algunos puntos de señal después de cada corte de la región de señal $\mathrm{SR}_{\mathrm{L}}$ (arriba) y $\mathrm{SR}_{\mathrm{H}}$ (abajo), para una luminosidad integrada de $20.3 \mathrm{fb}^{-1}$. Para los puntos de señal, también se muestra en la última fila, la significancia esperada.

\begin{tabular}{|c|c|c|c|c|c|c|c|c|c|}
\hline $\mathrm{SR}_{\mathrm{L}}$ & $(1150,200)$ & $(1150,450)$ & $\mathrm{V}_{\gamma}$ & Diboson & $\mathrm{V}+$ jets & + jets, multijet & $t \bar{t}$ & $t \bar{t} \gamma$ & Fondo total \\
\hline$=1$ fotón & 22.87 & 33.75 & 8396.04 & 230.19 & 10093.10 & 3103339.25 & 251.46 & 847.09 & $3123157.13 \pm 2013.86$ \\
\hline 0 leptones & 13.66 & 20.88 & 5433.21 & 166.08 & 7808.01 & 3101910.25 & 204.99 & 442.29 & $3115964.83 \pm 1971.53$ \\
\hline$E_{\mathrm{T}}^{\mathrm{mins}}$ & 7.74 & 14.96 & 457.80 & 11.07 & 188.39 & 94.27 & 6.50 & 22.21 & $780.24 \pm 55.42$ \\
\hline$N_{\text {jeti }}$ & 7.72 & 14.88 & 13.83 & 1.17 & 0.00 & 8.17 & 2.52 & 8.08 & $33.76 \pm 12.09$ \\
\hline$p_{\mathrm{T}}^{\mathrm{jet}_{1}}$ & 7.72 & 14.86 & 12.97 & 1.17 & 0.00 & 8.17 & 2.44 & 7.44 & $32.19 \pm 11.83$ \\
\hline$p_{\mathrm{T}}^{\text {jet }_{2}}$ & 7.68 & 14.71 & 9.00 & 1.14 & 0.00 & 7.94 & 1.97 & 5.63 & $25.69 \pm 10.66$ \\
\hline$R_{T}^{4}$ & 5.27 & 10.09 & 0.13 & 0.00 & 0.00 & 0.00 & 0.16 & 0.46 & $0.75 \pm 1.46$ \\
\hline Significancia & 3.81 & 6.14 & & & & & & & \\
\hline $\mathbf{S R}_{\mathrm{H}}$ & $(1150,850)$ & $(1150,1050)$ & $\mathrm{V} \gamma$ & Diboson & $V+$ jets & $\gamma+$ jets,multijet & $t \bar{t}$ & $t \bar{t} \gamma$ & Fondo total \\
\hline$=1$ fotón & 26.87 & 11.68 & 352.27 & 6.86 & 153.18 & 79255.07 & 0.73 & 49.23 & $79817.34 \pm 323.16$ \\
\hline 0 leptones & 22.92 & 11.20 & 215.86 & 4.40 & 102.50 & 79161.53 & 0.53 & 23.77 & $79508.59 \pm 313.87$ \\
\hline$\Delta \phi($ jet,$\gamma)$ & 9.20 & 5.18 & 3.21 & 0.22 & 0.00 & 0.00 & 0.00 & 0.30 & $3.73 \pm 2.82$ \\
\hline$H_{\mathrm{T}}$ & 8.67 & 4.79 & 0.66 & 0.00 & 0.00 & 0.00 & 0.00 & 0.10 & $0.76 \pm 1.14$ \\
\hline Significancia & 5.50 & 3.54 & & & & & & & \\
\hline
\end{tabular}
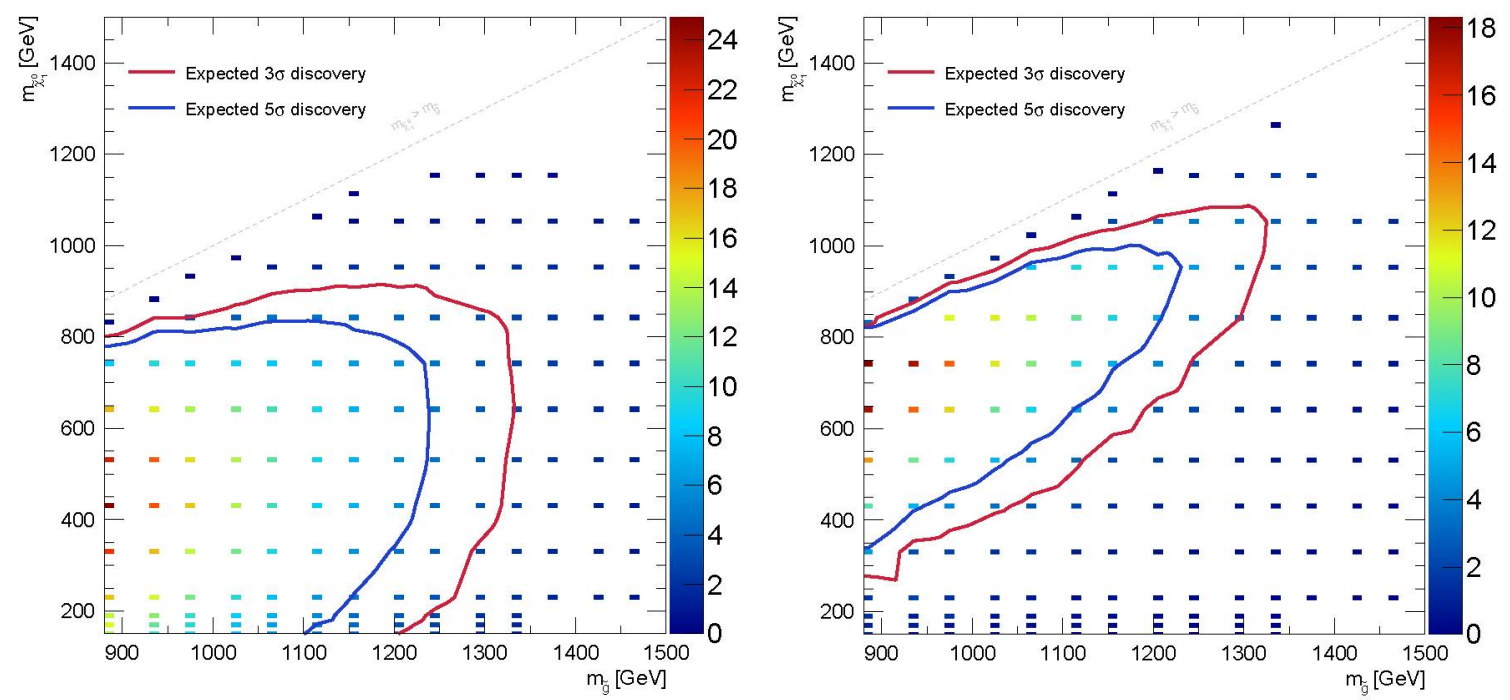

Figura 7.12: Significancia de descubrimiento esperada para todos los puntos de la grid de señal en el plano $\left(m_{\tilde{g}}, m_{\widetilde{\chi}_{1}^{0}}\right)$ para las dos regiones de señal $\mathrm{SR}_{\mathrm{L}}$ (izquierda) y $\mathrm{SR}_{\mathrm{H}}$ (derecha), junto con los contornos de $3 \sigma$ y $5 \sigma$.

donde $N_{\text {fiducial }}$ es el número de eventos que pasan los cortes fiduciales basados en los objetos a nivel generador incluyendo los cortes de $p_{\mathrm{T}} \mathrm{y} \eta$ del análisis. Además, se remueven los objetos superpuestos en el espacio $(\eta, \phi)$ como se describe en sección 7.3.3. $N_{\text {total }}$ es el número total de eventos.

La eficiencia se define como:

$$
\epsilon=\frac{N_{\text {fiducial,reco }}}{N_{\text {fiducial }}}
$$

donde $N_{\text {fiducial,reco }}$ es el número de eventos que pasan los cortes nominales del análisis aplicados a las variables a nivel detector. La eficiencia se diferencia de la aceptancia ya que incluye los efectos que 

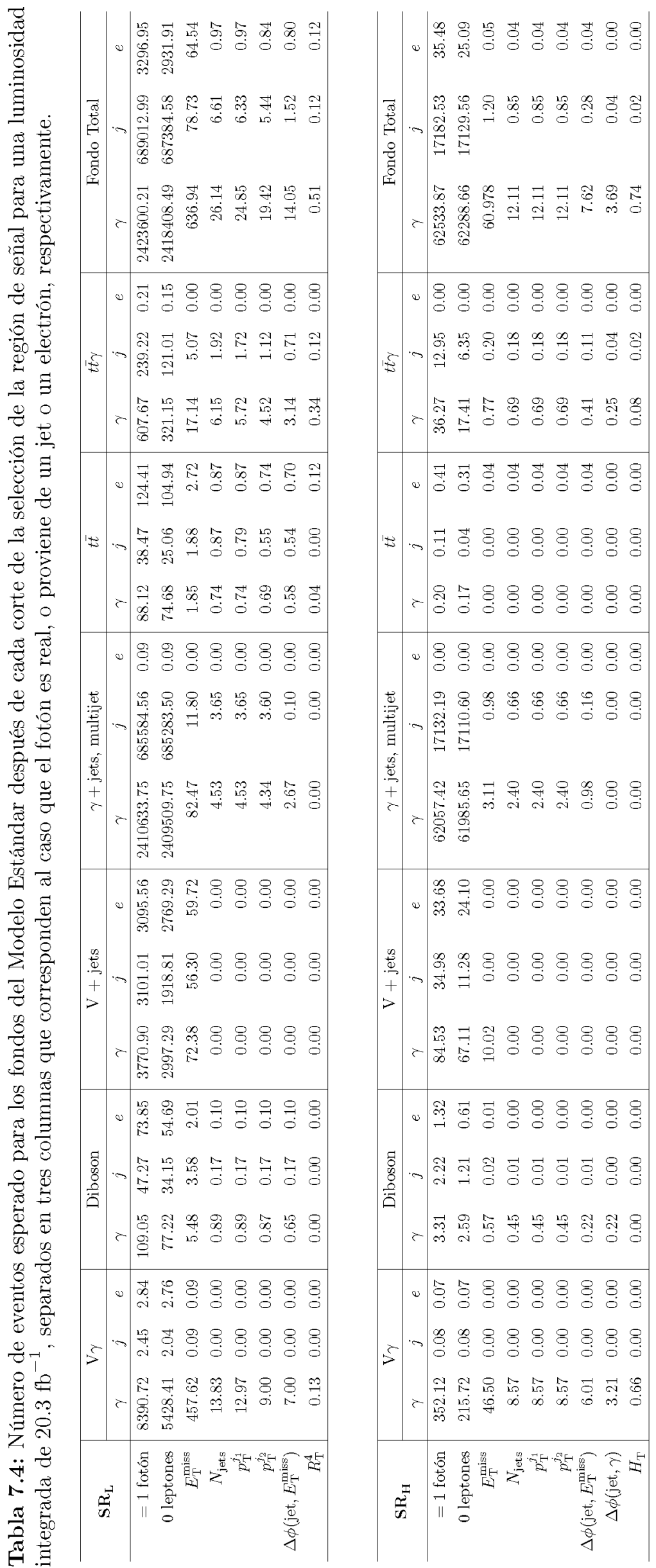
provienen de las ineficiencias de reconstrucción, los cortes de identificación de partículas, y los efectos de resolución e ineficiencias del trigger.

La aceptancia por la eficiencia $(A \times \epsilon)$ fue calculada para las dos regiones a partir de las muestras $\mathrm{MC}$, para asegurar que en el proceso de optimización se tuvieron en cuenta todas las regiones del espacio de parámetros, y evitar caídas bruscas en la eficiencia de selección. En la Figura 7.13 se puede ver que hay una buena cobertura de toda la grid de señal.
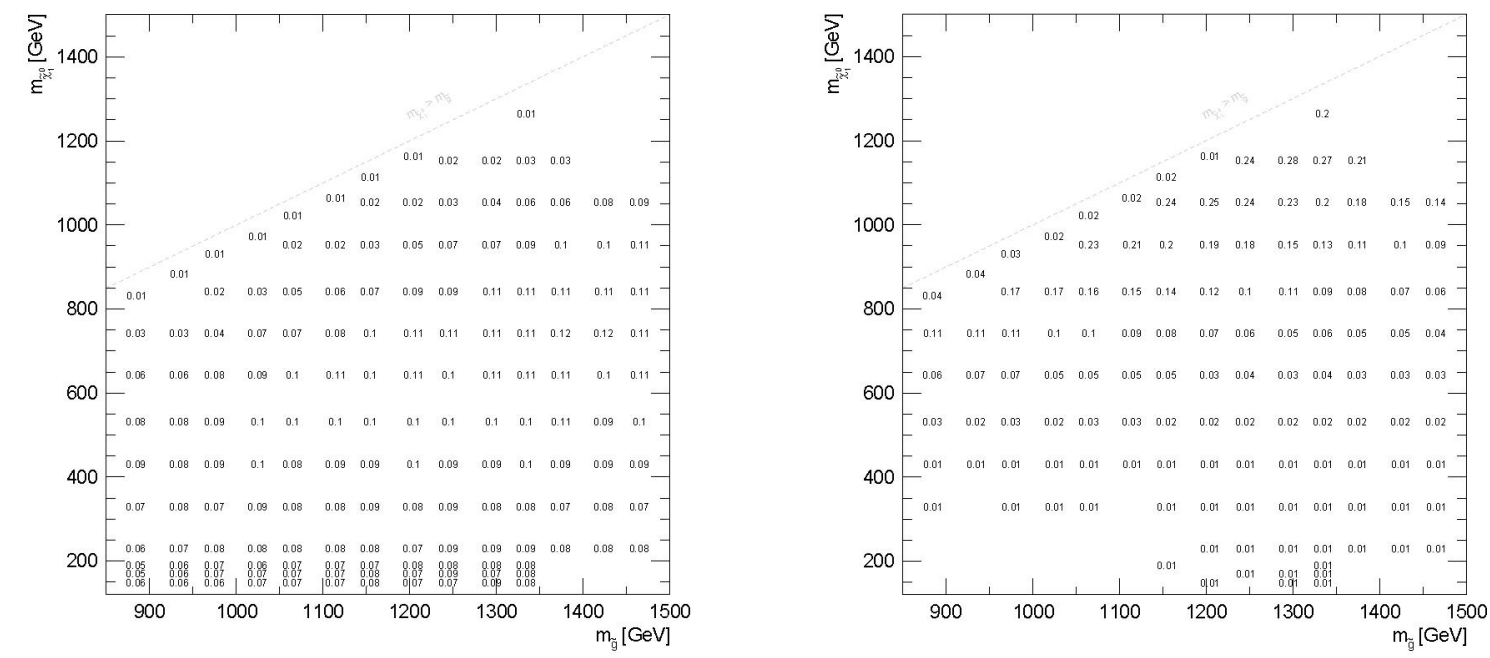

Figura 7.13: Producto de la aceptancia y la eficiencia $(A \times \epsilon)$ de selección para cada punto de señal en el plano $\left(m_{\tilde{g}}, m_{\widetilde{\chi}_{1}^{0}}\right)$ para la $\mathrm{SR}_{\mathrm{L}}$ (izquierda) y $\mathrm{SR}_{\mathrm{H}}$ (derecha). 


\section{ESTIMACIÓN DE LOS FONDOS}

Como se describió en la sección 7.5 se estima, utilizando las muestras MC, que la contribución dominante de fondos del Modelo Estándar es la debida a la producción de $W \gamma$ y $t \bar{t} \gamma$. Además en la $\mathrm{SR}_{\mathrm{L}}$ también se espera una contribución de procesos de $t \bar{t}$ en los que un electrón es mal identificado como fotón.

En este capítulo se describe la estrategia utilizada para la estimación de los fondos contaminantes provenientes de procesos del SM. Los fondos provenientes de electrones o jets mal identificados como fotones son estimados a partir de los datos observados como se describe en las secciones 8.3 y 8.4 , respectivamente. Para los fondos más importantes, $W \gamma$ y $t \bar{t} \gamma$, como no es posible obtener una estimación a partir de los mismos datos, se utilizan las muestras simuladas por MC pero corregidas usando los datos en una región de control, como se explica en la sección 8.1. La misma técnica se utiliza para el fondo de $\gamma+$ jets que, a pesar de ser muy pequeñas en las SR, si la energía faltante producida por la mala reconstrucción de los jets es elevada, puede ser relevante (ver sección 8.2). Las demás contribuciones, que son despreciables en las regiones de señal, se estimaron directamente de las muestras MC.

\subsection{Producción de $W \gamma$ y $t \bar{t} \gamma$}

Los eventos provenientes de la producción de $W \gamma$ y $t \bar{t} \gamma$ son las contribuciones dominantes al fondo en ambas regiones de señal.

La producción de $W \gamma$ dominante es básicamente $p p \rightarrow W \gamma+X \rightarrow \nu_{l} \bar{l} \gamma+X$. Cuando el leptón no es reconstruido o se pierde por la aceptancia del detector, pueden entrar en la SR. En el caso que el $W$ decaiga hadrónicamente no hay energía faltante y se espera de simulaciones MC que su contribución sea despreciable. Para la estimación final se considera esta contribución a partir de las muestras MC $(V(\rightarrow q q) \gamma)$.

En el caso de $t \bar{t} \gamma$, cada quark top decae en un bosón $W$ y un b-jet. Si uno de los $W$ decae leptónicamente y el leptón no es identificado ocurre lo mismo que en el caso de $W \gamma$ y puede contaminar la SR.

Se definen entonces dos regiones de control para determinar la normalización del MC de $W \gamma(\mathrm{CRW})$ y $t \bar{t} \gamma$ (CRT). Cada una de estas regiones de control es diseñada para que esté dominada por cada uno de estos fondos, para lo cual se pide un fotón, un leptón, jets y $E_{\mathrm{T}}^{\mathrm{miss}}$. Para CRW se pide además que no haya $b$-jets en el evento para reducir la contaminación de $t \bar{t} \gamma$, mientras que para CRT se requiere la presencia de al menos uno. Los cortes de selección se mantienen lo más similares posibles a la correspondiente SR para minimizar el efecto de la extrapolación, mientras que se remueven o relajan algunos cortes para aumentar la estadística. En especial, se relaja el corte en $E_{\mathrm{T}}^{\text {miss }}$ y no se aplican los cortes en $H_{\mathrm{T}}$ y en $R_{\mathrm{T}}^{4}$. Un esquema muy simple de estas CR puede verse en Figura 8.1. 
La selección completa de cada CR se presenta en la Tabla 8.1. Se utilizan dos regiones de control asociadas a cada SR.

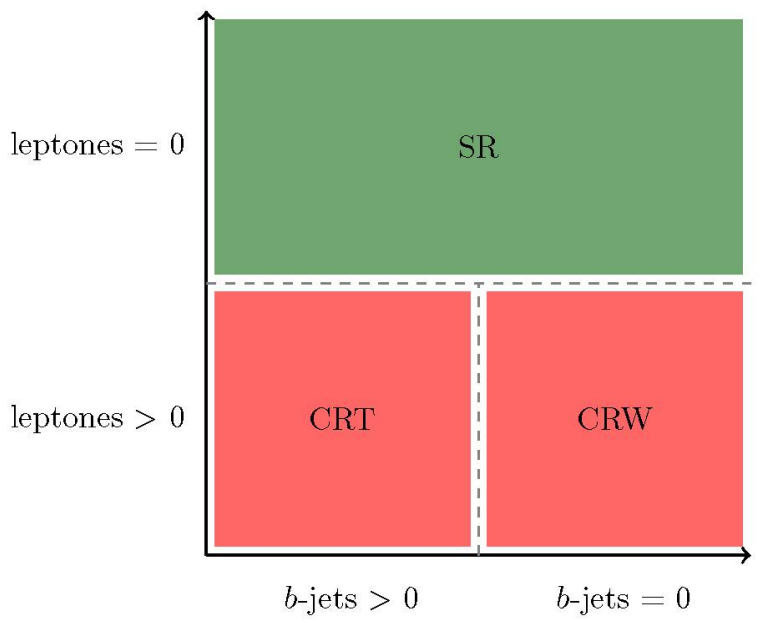

Figura 8.1: Regiones de control definidas para normalizar el fondo de $W \gamma$ (CRW) y $t \bar{t} \gamma$ (CRT). En este contexto "leptones" se refiere solo a electrones o muones.

Tabla 8.1: Selección para las regiones de control utilizadas para normalizar los fondos de $W \gamma, t \bar{t} \gamma \mathrm{y}$ $\gamma+$ jets, asociadas a las regiones de señal $\mathrm{SR}_{\mathrm{L}}$ y $\mathrm{SR}_{\mathrm{H}}$

\begin{tabular}{r|ccc|ccc}
\hline & \multicolumn{3}{|c|}{$\mathrm{SR}_{\mathrm{L}}$} & \multicolumn{3}{c}{$\mathrm{SR}_{\mathrm{H}}$} \\
\cline { 2 - 7 } & $\mathrm{CRW}_{\mathrm{L}}$ & $\mathrm{CRT}_{\mathrm{L}}$ & $\mathrm{CRQ}_{\mathrm{L}}$ & $\mathrm{CRW}_{\mathrm{H}}$ & $\mathrm{CRT}_{\mathrm{H}}$ & $\mathrm{CRQ}_{\mathrm{H}}$ \\
\hline$p_{\mathrm{T}}^{\gamma}[\mathrm{GeV}]>$ & 125 & 125 & 125 & 150 & 150 & 300 \\
$N_{\text {leptones }}$ & 1 & $\geq 1$ & 0 & 1 & $\geq 1$ & 0 \\
$E_{\mathrm{T}}^{\text {miss }}[\mathrm{GeV}]$ & {$[100-200]$} & {$[80-200]$} & $<50$ & {$[100-200]$} & {$[80-200]$} & $<50$ \\
$N_{\text {jets }} \geq$ & 4 & 4 & 4 & 2 & 2 & 2 \\
$N_{b-\text { jets }}$ & 0 & $\geq 1$ & - & 0 & $\geq 1$ & - \\
$p_{\mathrm{T}}^{j_{1}}, p_{\mathrm{T}}^{j_{2}}[\mathrm{GeV}]>$ & 100 & 100 & 100 & 40 & 40 & 40 \\
$\Delta \phi\left(\mathrm{jet}, E_{\mathrm{T}}^{\mathrm{miss}}\right)>$ & 0.4 & 0.4 & 0.4 & 0.4 & 0.4 & 0.4 \\
$R_{\mathrm{T}}^{4}<$ & - & - & 0.85 & - & - & - \\
$H_{\mathrm{T}}[\mathrm{GeV}]>$ & - & - & - & - & - & 800 \\
$\Delta \phi(\mathrm{jet}, \gamma)<$ & - & - & - & 2.0 & 2.0 & 2.0 \\
\hline
\end{tabular}

Es importante que las CR no tengan una contaminación de señal y para tal fin se aplica, además, un corte superior en $E_{\mathrm{T}}^{\text {miss }}$. En CRW y CRT la contaminación de señal en las CR es $<3 \%$ para la mayor parte del espacio de parámetros considerado, aunque es mayor (hasta 70\%) para algunos puntos de baja masa de gluino en $\mathrm{CRT}_{\mathrm{L}}$ (ver Figura 8.2).

\subsubsection{Regiones de validación}

Como se mencionó anteriormente, en la definición de las regiones de control, algunos cortes fueron relajados, o directamente removidos respecto a las $\mathrm{SR}$, para incrementar el número de eventos en las 

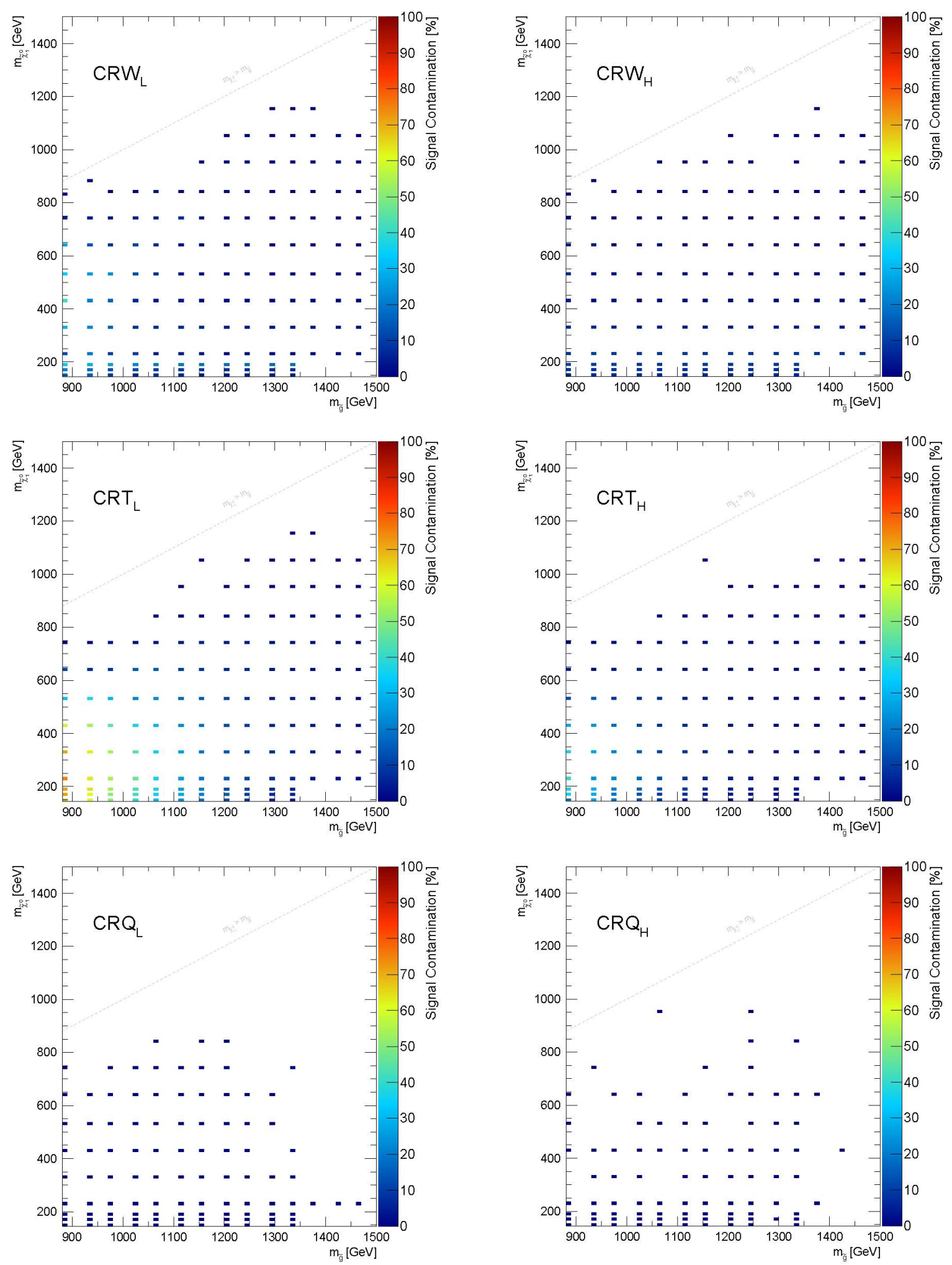

Figura 8.2: Contaminación de señal esperada en las regiones de control CRW (arriba), CRT (medio) $\mathrm{CRQ}$ (abajo) asociadas a $\mathrm{SR}_{\mathrm{L}}$ (izquierda) y $\mathrm{SR}_{\mathrm{H}}$ (derecha). 
mismas. Para validar la extrapolación entre las CR y la SR, se definen ciertas regiones de validación, imponiendo nuevamente los cortes de la SR, de a uno a la vez.

De esta forma se definen las siguientes regiones de validación para CRW y CRT, donde la X se refiere a la correspondiente CR (W o T). La selección detallada puede verse en Tabla 8.2.

VRXM igual que CRX pero con el corte en $E_{\mathrm{T}}^{\mathrm{miss}}$ como en la SR.

VRXR igual que CRX pero con el corte en $R_{\mathrm{T}}^{4}$ como en la $\mathrm{SR}_{\mathrm{L}}$ (solo para $\mathrm{SR}_{\mathrm{L}}$ ).

VRXH igual que CRX pero con el corte en $H_{\mathrm{T}}$ como en la $\mathrm{SR}_{\mathrm{H}}$ (solo para $\left.\mathrm{SR}_{\mathrm{H}}\right)$.

Tabla 8.2: Selección para las regiones de validación utilizadas para validar la extrapolación de los fondos de $W \gamma, t \bar{t} \gamma$, entre las $\mathrm{CR}$ a las regiones de señal $\mathrm{SR}_{\mathrm{L}}$ y $\mathrm{SR}_{\mathrm{H}}$

\begin{tabular}{r|cccc|cccc}
\hline & \multicolumn{4}{|c|}{$\mathrm{SR}_{\mathrm{L}}$} & \multicolumn{5}{c}{$\mathrm{SR}_{\mathrm{H}}$} \\
\cline { 2 - 9 } & VRWM & VRWR & VRTM & VRTR & VRWM & VRWH & VRTM & VRTH \\
\hline$p_{\mathrm{T}}^{\gamma}[\mathrm{GeV}]>$ & 125 & 125 & 125 & 125 & 150 & 150 & 150 & 150 \\
$N_{\text {leptones }}$ & 1 & 1 & $\geq 1$ & $\geq 1$ & 1 & 1 & $\geq 1$ & $\geq 1$ \\
$E_{\mathrm{T}}^{\text {miss }}[\mathrm{GeV}]$ & $>200$ & {$[100-200]$} & $>200$ & {$[80-200]$} & $>300$ & {$[100-200]$} & $>300$ & {$[80-200]$} \\
$N_{\text {jets }} \geq$ & 4 & 4 & 4 & 4 & 2 & 2 & 2 & 2 \\
$N_{b-\text { jets }}$ & 0 & 0 & $\geq 1$ & $\geq 1$ & 0 & 0 & $\geq 1$ & $\geq 1$ \\
$p_{\mathrm{T}}^{j_{1}}, p_{\mathrm{T}}^{j_{2}}[\mathrm{GeV}]>$ & 100 & 100 & 100 & 100 & 40 & 40 & 40 & 40 \\
$\Delta \phi\left(\mathrm{jet}, E_{\mathrm{T}}^{\text {miss }}\right)>$ & 0.4 & 0.4 & 0.4 & 0.4 & 0.4 & 0.4 & 0.4 & 0.4 \\
$R_{\mathrm{T}}^{4}<$ & - & 0.85 & - & 0.85 & - & - & - & - \\
$H_{\mathrm{T}}[\mathrm{GeV}]>$ & - & - & - & - & - & 800 & - & 800 \\
\hline
\end{tabular}

\subsection{Producción de fotones directos $(\gamma+$ jets $)$}

Por diseño de la selección, la probabilidad de detectar eventos de procesos de producción de fotones directos $(\gamma+$ jets $)$ en la región de señal es baja, ya que resulta raro que estos eventos tengan una gran cantidad de $E_{\mathrm{T}}^{\text {miss }}$. Sin embargo, esta energía faltante puede ser producida por la mala reconstrucción de la energía de los jets y debido a que la sección eficaz de estos procesos es muy alta, puede resultar en una contaminación significativa.

Para determinar este fondo no es posible confiar plenamente en las simulaciones MC, debido a que la baja cantidad de eventos similares a la señal implicaría usar una muestra con un número muy alto de eventos, lo que resulta dificultoso desde un punto de vista computacional. Por este motivo se diseñó una región de control dominada por eventos de $\gamma+$ jets a la que se llamó CRQ. Los detalles de la selección se pueden ver en la Tabla 8.1. Básicamente es la misma selección que la SR pero requiriendo baja energía faltante $\left(E_{\mathrm{T}}^{\text {miss }}<50 \mathrm{GeV}\right)$.

La contaminación de señal en esta región de control es despreciable, debido a que la señal posee gran cantidad de energía faltante, como puede verse en la Figura 8.2.

\subsubsection{Regiones de validación}

Con un conjunto de cortes de selección se definen regiones para validar los resultados de la extrapolación de $\gamma+$ jets de la región de control a bajo $E_{\mathrm{T}}^{\text {miss }}$ hasta las $\mathrm{SR}$ a alto $E_{\mathrm{T}}^{\text {miss }}$. Las regiones 
de validación deben estar lo más cerca posible de las SR en el espacio de observables, pero deben ser ortogonales a las mismas. Esto se logra invirtiendo algunos cortes, como se describe a continuación.

VRMX igual a la SR pero con un requerimiento intermedio en $E_{\mathrm{T}}^{\text {miss }}\left(X \mathrm{GeV}<E_{\mathrm{T}}^{\text {miss }}<150 \mathrm{GeV}\right)$ con $X=75,100$. El corte superior asegura la ortogonalidad con la SR.

VRH igual a la SR pero invirtiendo el corte en $H_{\mathrm{T}}\left(H_{\mathrm{T}}<800 \mathrm{GeV}\right)$. Solo definida para $\mathrm{SR}_{\mathrm{H}}$, ya que no hay un corte en $H_{\mathrm{T}}$ en $\mathrm{SR}_{\mathrm{L}}$.

VRQ igual a la SR pero invirtiendo el corte en $\Delta \phi\left(\right.$ jet, $\left.E_{\mathrm{T}}^{\text {miss }}\right)\left(\Delta \phi\left(\right.\right.$ jet, $\left.\left.E_{\mathrm{T}}^{\text {miss }}\right)<0.4\right)$ para aumentar la contribución de fondos con $E_{\mathrm{T}}^{\text {miss }}$ instrumental.

En la Figura 8.3 pueden verse los esquemas de la definición de las CR y VR.
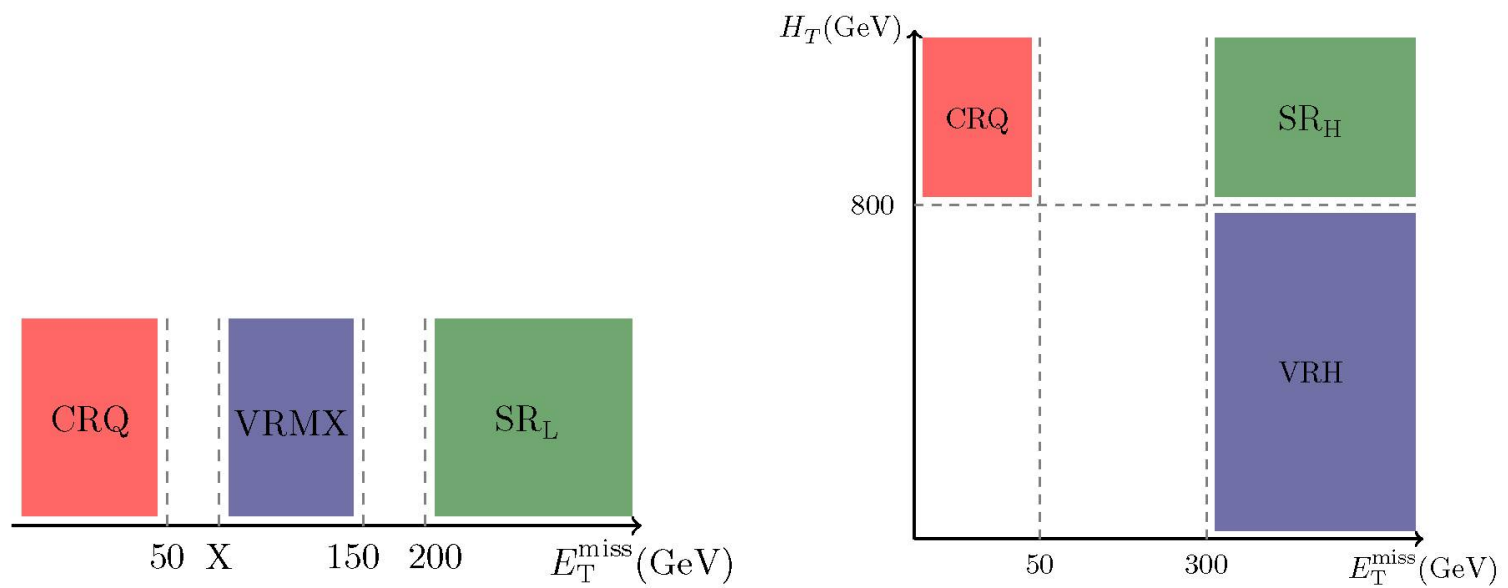

Figura 8.3: Esquema de las regiones de control diseñadas para normalizar el fondo de $\gamma+$ jets para $\mathrm{SR}_{\mathrm{L}}$ (izquierda) y $\mathrm{SR}_{\mathrm{H}}$ (derecha). También se muestran las regiones de validación utilizadas para validar la extrapolación $\mathrm{CR} \rightarrow \mathrm{SR}$.

\subsection{Electrones identificados como fotones}

Los eventos en los que un electrón de alto $p_{\mathrm{T}}$ es identificado como un fotón [149] pueden contaminar alguna de las SR. Esta contaminación proviene generalmente de procesos del SM como $W(\rightarrow e \nu)+$ jets, $Z(\rightarrow e e)+$ jets y $t \bar{t}$.

Como se mencionó en la sección 3.2, los electrones y fotones dejan lluvias electromagnéticas muy similares en el detector. Los algoritmos de reconstrucción de fotones están diseñados para reducir la identificación errónea de electrones como fotones aunque, para poder mantener una alta eficiencia de reconstrucción, no se realiza una distinción demasiado estricta. En caso de duda, los clusters electromagnéticos son reconstruidos bajo ambas hipótesis (electrón o fotón) y guardados (duplicados) en ambas categorías. Esto implica que algunos electrones pueden terminar siendo reconstruidos como fotones, y si estos pasan la selección de fotones utilizada en el análisis, contribuirán al fondo por mala identificación $e \rightarrow \gamma$.

Los electrones son reconstruidos a partir de los clusters asociados a una traza, mientras que los fotones son reconstruidos de los clusters que no tienen ninguna traza asociada (candidatos a fotones no-convertidos) o están asociados a un vértice de conversión (candidatos a fotones convertidos). Se 
consideran los vértices con una o dos trazas en la reconstrucción de fotones convertidos, y por lo tanto los fotones convertidos pueden ser categorizados como fotones convertidos con una traza o dos trazas. La traza de los candidatos a fotón convertidos con una sola traza debe poseer un impacto en la capa- $B$ del detector interno. Se espera que una fracción de electrones pueda ser reconstruida como fotones convertidos, por ejemplo, si falla la asociación de la traza a un impacto en la capa- $B$, o si se asocia un vértice de conversión espurio a ella. Asimismo, el electrón puede ser reconstruido como un fotón no-convertido si falla el algoritmo que asocia la traza al cluster.

La fracción de electrones reconstruidos como fotones, depende claramente del material en el detector, es por eso que pueden existir diferencias si se calcula a partir de simulaciones Monte Carlo, ya que el material en las simulaciones no es perfecto. Por tal motivo la fracción de electrones reconstruida como fotones se obtiene a partir de los datos, aunque también se realiza una comparación con las muestras MC. El número de eventos de electrones reconstruidos como fotones en la región de señal $\left(N_{e \rightarrow \gamma}^{\mathrm{SR}}\right)$ se obtiene multiplicando esta fracción $\left(f_{e \rightarrow \gamma}\right)$ por el número de eventos en la muestra de electrones obtenida invirtiendo el rol de fotones y electrones en la selección de la región de señal $\left(N_{e}^{\mathrm{SR}}\right)$. Para calcular este número de eventos se requiere un electrón aislado de alto $p_{\mathrm{T}}, \mathrm{y}$ los fotones de señal son vetados.

$$
N_{e \rightarrow \gamma}^{\mathrm{SR}}=f_{e \rightarrow \gamma} \cdot N_{e}^{\mathrm{SR}}
$$

Para estimar $f_{e \rightarrow \gamma}$ se utiliza un método llamado «tag and probe», en una muestra de eventos de datos $Z \rightarrow e^{+} e^{-}$que pasan el mismo trigger de fotones que utiliza el análisis y la misma preselección. Adicionalmente, se aplica un corte de $E_{\mathrm{T}}^{\mathrm{miss}}<40 \mathrm{GeV}$ para reducir la posible contaminación de fotones reales de eventos de $W \gamma$.

El método consiste en seleccionar un electrón (el electrón tag) que pasa un criterio de identificación tight y que tiene $20 \mathrm{GeV}<p_{\mathrm{T}}<125 \mathrm{GeV}$. Luego se busca un segundo candidato a electrón o fotón (el objeto probe). Este puede ser un electrón tight o un fotón tight, ambos con $p_{\mathrm{T}}>125 \mathrm{GeV} \mathrm{y}$ satisfaciendo los requerimientos de aislamiento correspondientes.

Los valores de la masa de los pares de objetos seleccionados son guardados separadamente para los tres casos posibles: dos electrones, un electrón y un fotón convertido, o un electrón y fotón no-convertido. En los tres casos se puede ver un pico alrededor del valor de la masa del bosón $Z(\sim 91 \mathrm{GeV})$. Como el bosón $Z$ no puede decaer directamente en un electrón y un fotón, los pares electrón-fotón que aparecen bajo el pico del $Z$ corresponden a electrones mal identificados. Sin embargo, lo mismo aplica a otros procesos que contienen dos electrones en estado final. Por lo tanto es necesario utilizar algún método de sustracción del fondo. Este deberá también tener en cuenta la contaminación por las combinaciones aleatorias.

La probabilidad de identificación errónea $f_{e \rightarrow \gamma}$ se define entonces como:

$$
f_{e \rightarrow \gamma}=\frac{N_{e \gamma}}{N_{e e}}
$$

donde $N_{e \gamma}\left(N_{e e}\right)$ es el número de pares electrón-fotón (electrón-electrón) encontrados en una ventana alrededor del pico del bosón $Z$ en la distribución de la masa invariante, definida en el rango $81<$ $m_{e x}<101 \mathrm{GeV}$. Para obtener el número de eventos $N_{e x}$, se realiza un ajuste de la distribución de la masa invariante para los dos tipos de eventos utilizando un modelo de señal + fondo. Este procedimiento se lleva a cabo de forma separada para fotones convertidos y no-convertidos, y en clases del $|\eta|$ de los objetos probe. El bajo número de eventos disponibles hace imposible utilizar clases de $p_{\mathrm{T}}$, aunque de esta forma la estimación es conservativa ya que la probabilidad de identificación errónea decrece con el $p_{\mathrm{T}}$ del electrón [150].

Como modelo de señal se utiliza la suma de función Crystall-Ball y una gaussiana, mientras que para 
el modelo de fondo se utiliza un polinomio de grado dos. En la Figura 8.4 se pueden ver las distribuciones

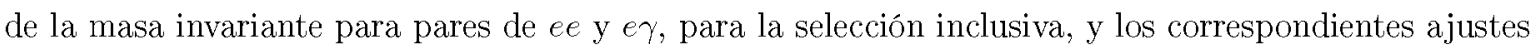
del modelo.
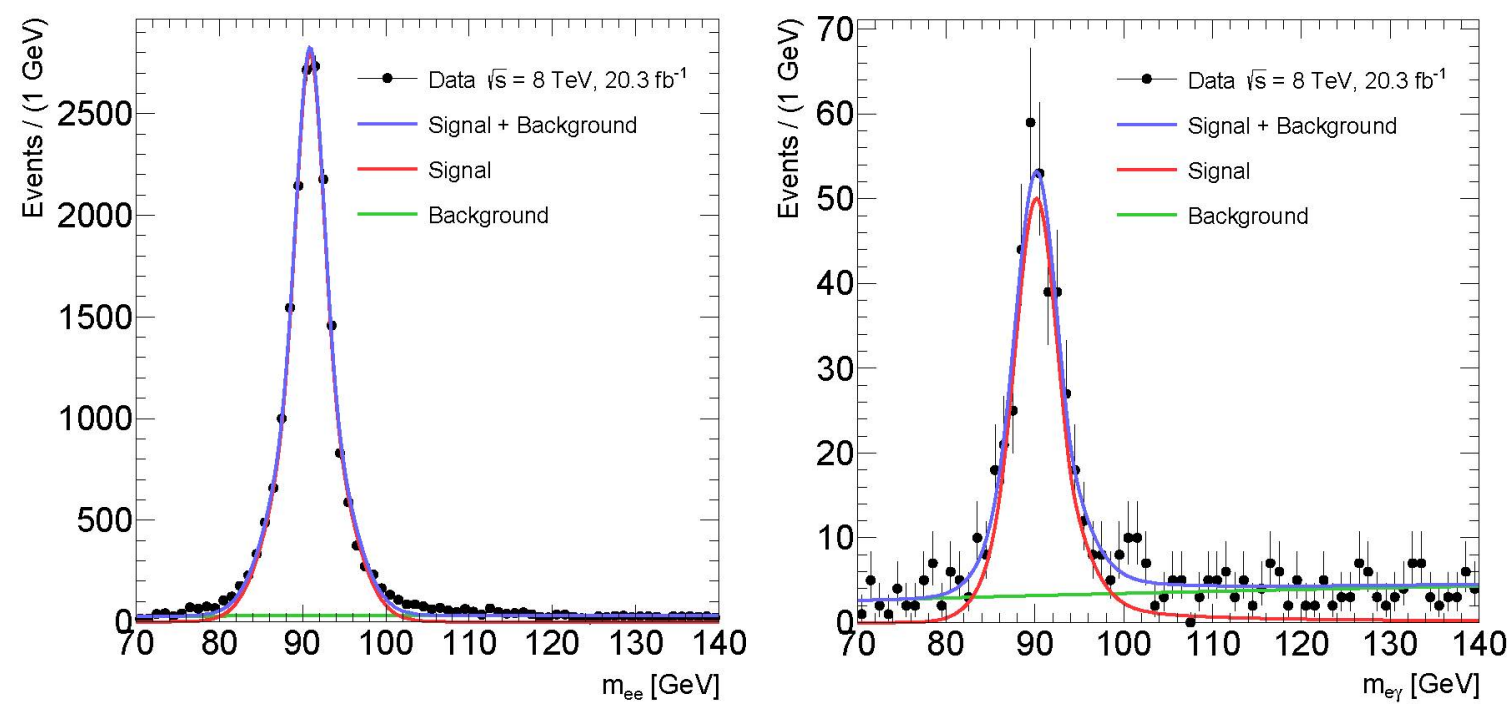

Figura 8.4: Distribuciones de la masa invariante de los pares de electrón-electrón (izquierda) y electrón-fotón (derecha). Se puede ver también el ajuste con el modelo de señal + fondo.

La probabilidad de identificación errónea se muestra en la Figura 8.5, como función del $|\eta|$ del objeto probe para "fotones" convertidos y no-convertidos. Este factor crece con $|\eta|$, dado que está relacionado con el incremento en el material del detector atravesado por los electrones y el incremento en la tasa de reconstrucción de fotones convertidos con una sola traza.

El $f_{e \rightarrow \gamma}$ calculado a partir de los datos es comparado con el calculado a partir de muestras MC de eventos de $Z \rightarrow e^{+} e^{-}$producidas con los generadores SHERPA y PowheG. Se encuentra un buen acuerdo para todos los casos dentro de sus incertezas. Los valores calculados de $f_{e \rightarrow \gamma}$ pueden verse en la Tabla 8.3, y Tabla 8.4 para fotones convertidos y no-convertidos de forma separada.

Tabla 8.3: Probabilidad de que un electrón real sea identificado como un fotón como función de la pseudo-rapidez del objeto probe. El valor calculado a partir de los datos es comparado con el valor calculado con las muestras MC de eventos de $Z \rightarrow e^{+} e^{-}$utilizando dos generadores distintos.

\begin{tabular}{cccc}
\hline Datos & $\begin{array}{c}\mathrm{MC} Z \rightarrow e^{+} e^{-} \\
\text {(SHERPA) }\end{array}$ & $\begin{array}{c}\mathrm{MC} Z \rightarrow e^{+} e^{-} \\
\text {(PowhEG) }\end{array}$ \\
\hline $0<|\eta|<0.8$ & $0.014 \pm 0.002$ & $0.012 \pm 0.001$ & $0.014 \pm 0.002$ \\
$0.8<|\eta|<1.52$ & $0.018 \pm 0.003$ & $0.014 \pm 0.001$ & $0.011 \pm 0.003$ \\
$1.52<|\eta|<2.5$ & $0.033 \pm 0.006$ & $0.027 \pm 0.002$ & $0.032 \pm 0.006$ \\
Inclusivo & $0.019 \pm 0.001$ & $0.016 \pm 0.001$ & $0.017 \pm 0.002$ \\
\hline
\end{tabular}

Para estimar la incerteza sistemática del método utilizado, el factor $f_{e \rightarrow \gamma}$ fue calculado variando el tamaño de la ventana de masa del $Z$, y sin la sustracción del fondo. Como se aprecia en la Tabla 8.5 la mayor variación se obtiene en el caso de no realizar la sustracción del fondo y por lo tanto se utiliza 

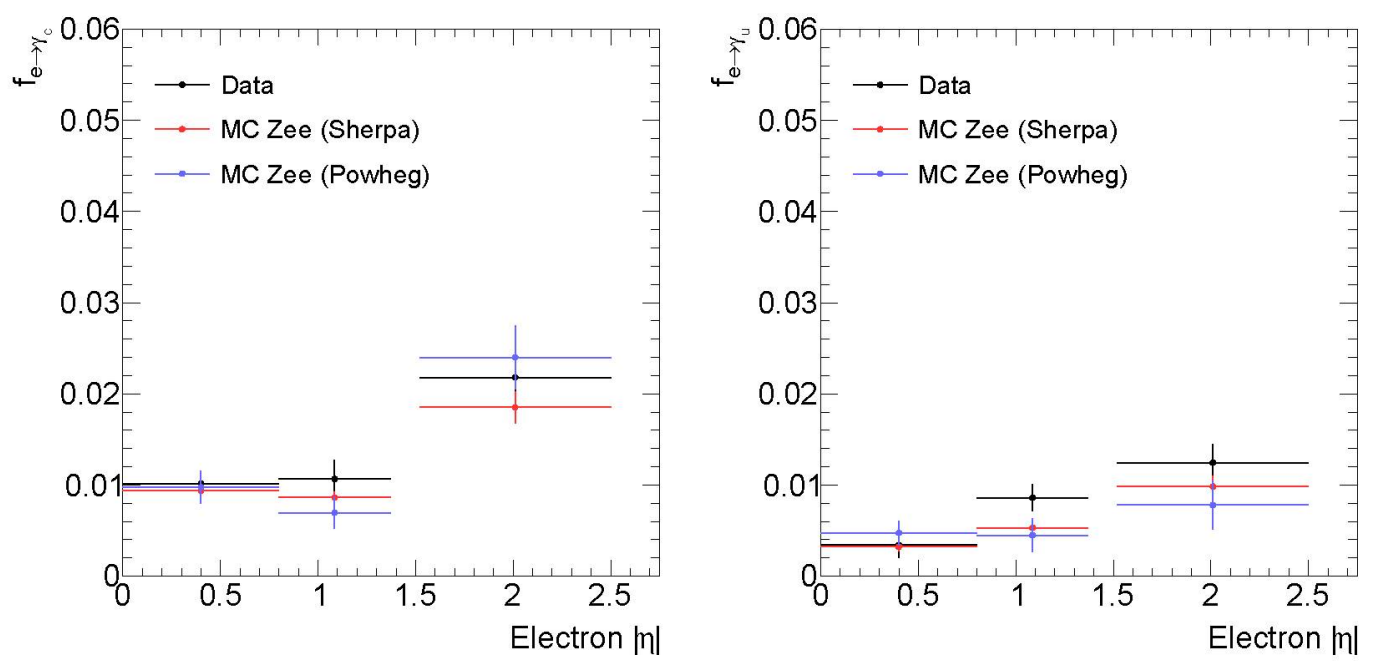

Figura 8.5: Probabilidad de que un electrón real sea identificado como un fotón convertido (izquierda) y un fotón no-convertido (derecha), como función de la pseudo-rapidez del objeto probe. El valor calculado a partir de los datos es comparado con el valor calculado con las muestras MC de eventos de $Z \rightarrow e^{+} e^{-}$utilizando dos generadores distintos.

Tabla 8.4: Probabilidad de que un electrón real sea reconstruido como un fotón convertido $\left(f_{e \rightarrow \gamma_{c}}\right)$ o no-convertido $\left(f_{e \rightarrow \gamma_{u}}\right)$. El valor calculado a partir de los datos es comparado con el valor calculado con las muestras MC de eventos de $Z \rightarrow e^{+} e^{-}$, utilizando dos generadores distintos.

\begin{tabular}{cccc}
\hline Datos & $\begin{array}{c}\mathrm{MC} Z \rightarrow e^{+} e^{-} \\
\text {(SHERPA) }\end{array}$ & $\begin{array}{c}\text { MC } Z \rightarrow e^{+} e^{-} \\
\text {(PowHEG) }\end{array}$ \\
\hline$f_{e \rightarrow \gamma_{u}}$ & $0.007 \pm 0.001$ & $0.005 \pm 0.001$ & $0.005 \pm 0.001$ \\
$f_{e \rightarrow \gamma_{c}}$ & $0.013 \pm 0.001$ & $0.011 \pm 0.001$ & $0.011 \pm 0.002$ \\
$f_{e \rightarrow \gamma}$ & $0.019 \pm 0.001$ & $0.016 \pm 0.001$ & $0.017 \pm 0.002$ \\
\hline
\end{tabular}

este valor como la incerteza sistemática del método, a pesar de que resulta el límite más desfavorable.

Tabla 8.5: Probabilidad de que un electrón real sea reconstruido como un fotón convertido o noconvertido, para variaciones del método original.

\begin{tabular}{cccc}
\hline & $m_{e e} \in[71,111] \mathrm{GeV}$ & $m_{e e} \in[86,96] \mathrm{GeV}$ & $\begin{array}{c}\text { Sin sustracción del } \\
\text { fondo }\end{array}$ \\
\hline$f_{e \rightarrow \gamma_{u}}$ & $0.007 \pm 0.001$ & $0.007 \pm 0.001$ & $0.012 \pm 0.001$ \\
$f_{e \rightarrow \gamma_{c}}$ & $0.013 \pm 0.001$ & $0.012 \pm 0.001$ & $0.012 \pm 0.001$ \\
$f_{e \rightarrow \gamma}$ & $0.019 \pm 0.001$ & $0.019 \pm 0.001$ & $0.024 \pm 0.001$ \\
\hline
\end{tabular}

El valor calculado del factor de identificación errónea de electrón en fotón $\left(f_{e \rightarrow \gamma}\right)$ resulta entonces el que se muestra en la Tabla 8.6.

En la $\mathrm{SR}_{\mathrm{L}}$ se observaron 28 eventos que pasaban la selección al invertir el rol de fotón y electrón, lo que corresponde a una estimación del fondo de $N_{e \rightarrow \gamma}^{\mathrm{SR}_{\mathrm{L}}}=0.38 \pm 0.10$. En $\mathrm{SR}_{\mathrm{H}}$, dado el alto corte en $E_{\mathrm{T}}^{\mathrm{miss}}$, no se observó ningún evento en la muestra de electrones. 
Tabla 8.6: Probabilidad de que un electrón real sea reconstruido como un fotón $f_{e \rightarrow \gamma}$, como función de $\eta$, junto con su incerteza estadística y sistemática.

\begin{tabular}{cc}
\hline Región & $f_{e \rightarrow \gamma}$ \\
\hline $0<|\eta|<0.8$ & $0.014 \pm 0.002$ (stat.) \pm 0.005 (sist.) \\
$0.8<|\eta|<1.52$ & $0.018 \pm 0.003$ (stat.) \pm 0.004 (sist.) \\
$1.52<|\eta|<2.5$ & $0.033 \pm 0.006$ (stat.) \pm 0.008 (sist.) \\
\hline
\end{tabular}

\subsection{Jets identificados como fotones}

Los jets pueden ser identificados como fotones si tienen uno o dos $\pi^{0}$ con alto $p_{\mathrm{T}}$ como partícula leading, resultando en un objeto electromagnético indistinguible de un fotón muy energético. Este fondo proviene mayoritariamente de multijets, $\mathrm{W}+$ jets y eventos $t \bar{t}$ decayendo semi-leptónicamente, $\mathrm{y}$ puede ser una fuente importante de contaminación debido a la gran sección eficaz de producción de estos procesos. Como la proporción de jets mal identificados como fotones no está bien descripta por el MC, se utiliza para su estimación un método especialmente desarrollado a partir de los datos. La idea consiste en utilizar las diferencias en la distribución de energía de aislamiento esperada para fotones reales y falsos, como se describe a continuación.

\subsubsection{Descripción del método}

Para reducir significativamente el fondo proveniente de jets, en el análisis se utilizan fotones que satisfacen un criterio de selección tight, como se describe en sección 3.2. Esta selección es inclusiva respecto a los fotones reales, tiene una moderada contaminación de jets y es, por definición, un criterio más ajustado que el trigger de fotones utilizado para la toma de los datos. Por esta razón, hay una suficiente cantidad de candidatos a fotones provenientes de jets que fallan la selección tight pero satisfacen una selección intermedia. Estos jets más similares a un fotón real, denominados «pseudo-fotones», son útiles para modelar los jets que pasan la selección total y la fracción de esta contaminación.

La muestra de fotones que pasan los criterios de selección tight $\left(N_{\text {tight }}\right)$ contiene, en general, fotones reales $\left(N_{\gamma}\right)$ y falsos $\left(N_{j \rightarrow \gamma}\right)$, es decir, $N_{t i g h t}=N_{\gamma}+N_{j \rightarrow \gamma}$. Las distribuciones de la energía de aislamiento para estas dos contribuciones van a tener formas diferentes, lo que puede ser explotado para estimar ambas contribuciones. Para tal fin, se ajusta la distribución total de energía de aislamiento con una combinación de modelos de señal y fondo.

El número de eventos de fotones falsos que pasa la identificación de fotones y el corte de aislamiento puede ser estimado entonces integrando la componente de fondo del ajuste sobre el rango de la región de señal $\left(E_{\mathrm{T}}^{\mathrm{iso}}<5 \mathrm{GeV}\right)$. De esta forma la proporción de jets mal identificados como fotones, $f_{j \rightarrow \gamma}$, resulta:

$$
f_{j \rightarrow \gamma}=\frac{\int_{-\infty}^{5 \mathrm{GeV}} B(x) d x}{\int_{-\infty}^{5 \mathrm{GeV}}[S(x)+B(x)] d x}
$$

donde $x$ es la variable de energía de aislamiento $E_{\mathrm{T}}^{\text {iso }}$ y $S(x)$ y $B(x)$ son las distribuciones de señal y fondo en el ajuste combinado, respectivamente. Esta fracción de fotones falsos es estimada en una región lo más cercana posible a las regiones de señal, y luego utilizada para estimar el número de eventos provenientes de jets mal identificados, pesando la muestra de fotones en la SR. 


\subsubsection{Modelo de señal}

El modelo de señal se extrajo a partir de electrones provenientes de eventos de datos $Z \rightarrow e^{+} e^{-}$ utilizando el hecho que los electrones y fotones dejan una señal similar en el calorímetro electromagnético. La muestra de electrones es obtenida de eventos que satisfacen el siguiente conjunto de cortes, después de haber pasado la preselección descripta en sección 7.3.2:

- Trigger de electrones

- Dos electrones medium, aislados, con carga opuesta, y con $p_{\mathrm{T}}>50,25 \mathrm{GeV}$

- $E_{\mathrm{T}}^{\mathrm{miss}}<40 \mathrm{GeV}$

- $81 \mathrm{GeV}<m_{e e}<101 \mathrm{GeV}$

Después de aplicar los cortes anteriores, la contaminación de fondos no provenientes del decaimiento del $Z$ es despreciable y, en particular, para eventos de $t \bar{t}$ resulta menor al $1 \%$.

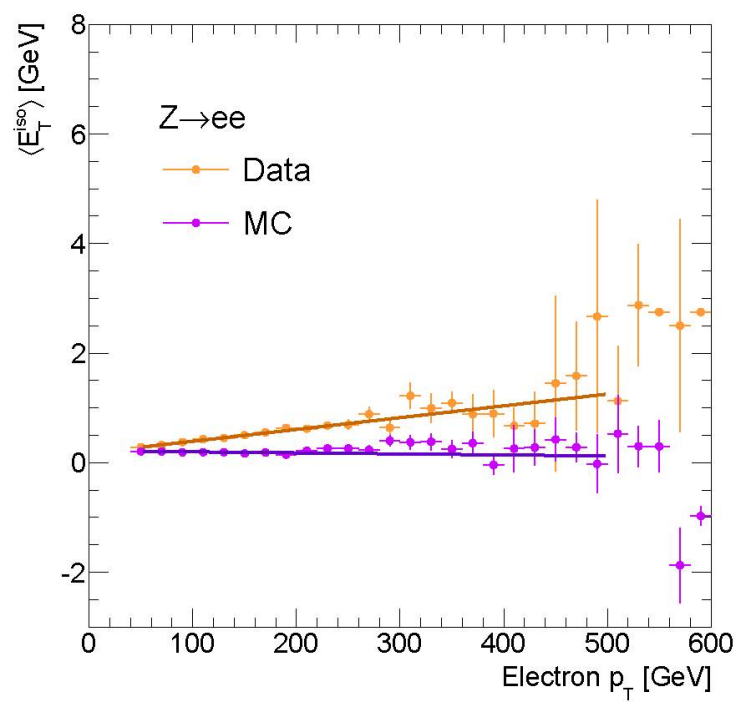

Figura 8.6: Distribución de la energía de aislamiento $\left(E_{\mathrm{T}}^{\mathrm{iso}}\right)$ de electrones provenientes de eventos $Z \rightarrow e^{+} e^{-}$en datos y MC, en función de $p_{\mathrm{T}}$.

La Figura 8.6 muestra el valor medio de la distribución de la energía de aislamiento para los electrones seleccionados como función del $p_{\mathrm{T}}$ del electrón en eventos de datos y simulaciones $\mathrm{MC}$. De la figura resulta evidente que para los datos existe una clara dependencia de $E_{\mathrm{T}}^{\text {iso }}$ con $p_{\mathrm{T}}$, que no es el caso para las simulaciones MC. Para corregir esta dependencia residual con $p_{\mathrm{T}}$ se realiza un ajuste lineal de los datos del cual se obtiene un factor de corrección que luego es aplicado a $E_{\mathrm{T}}^{\text {iso }}$. El valor del factor de corrección obtenido en el ajuste en el rango $50<p_{\mathrm{T}}<500 \mathrm{GeV}$ es $0.00262 \pm 0.00008$.

En la Figura 8.7 se muestra una comparación entre datos y simulaciones del perfil de $E_{\mathrm{T}}^{\text {iso }}$ antes y después de la corrección. Se puede ver que el acuerdo entre datos y MC es mucho mejor después de aplicar la corrección.

Para respaldar la estrategia de derivar el modelo de aislamiento de los fotones a partir de electrones se realizaron varios estudios. Una validación importante del método consistió en comparar la distribución de $E_{\mathrm{T}}^{\mathrm{iso}}$ de electrones provenientes de $Z \rightarrow e^{+} e^{-}$con los fotones provenientes del decaimiento radiativo 

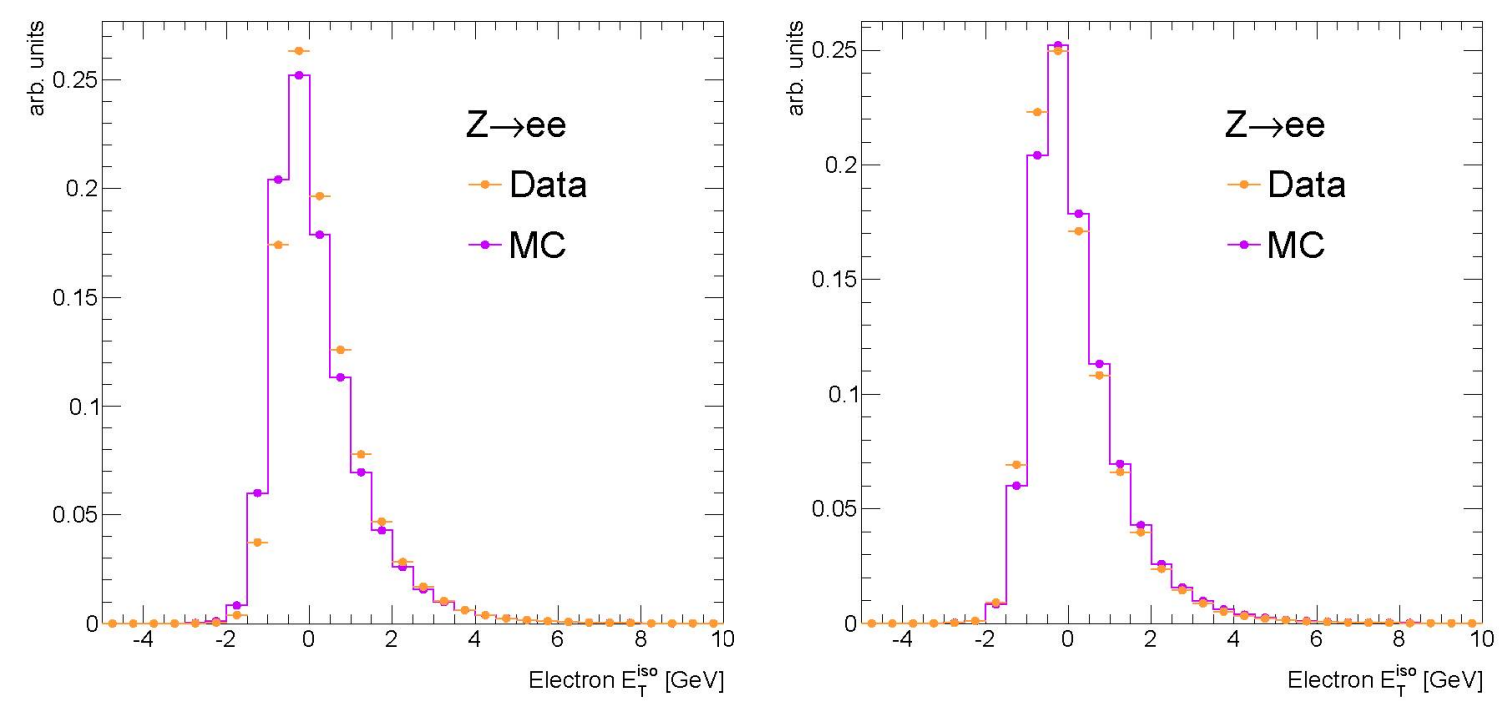

Figura 8.7: Comparación datos/MC de la distribución de $E_{\mathrm{T}}^{\text {iso }}$ de electrones provenientes de eventos $Z \rightarrow e^{+} e^{-}$(izquierda) y la correspondiente distribución después de aplicar la corrección por el $p_{\mathrm{T}}$ (derecha).

del $Z\left(Z \rightarrow e^{+} e^{-} \gamma\right)$ que provee un fuente de fotones puros. Los eventos se seleccionaron requiriendo el siguiente conjunto de cortes, después de la preselección:

- Triggers de electrones

- Un fotón tight aislado con $p_{\mathrm{T}}>25 \mathrm{GeV}$.

- Dos electrones medium, aislados, con carga opuesta, y con $p_{\mathrm{T}}>50 \mathrm{GeV}$ y $p_{\mathrm{T}}>25 \mathrm{GeV}$

- $\Delta R(\gamma, l)>0.7$

- $E_{\mathrm{T}}^{\mathrm{miss}}<40 \mathrm{GeV}$

- $40 \mathrm{GeV}<m_{e e}<85 \mathrm{GeV}$

- $70 \mathrm{GeV}<m_{e e \gamma}<100 \mathrm{GeV}$

En la Figura 8.8 se presenta una comparación entre el modelo de fotones reales de decaimientos radiativos del $Z$ y electrones de $Z \rightarrow e^{+} e^{-}$. El gráfico a la derecha es obtenido después de remover la dependencia con el $p_{\mathrm{T}}$ con el factor de corrección como se describió anteriormente. Puede verse, a partir del cociente en el panel inferior, el buen acuerdo entre las distribuciones de $E_{\mathrm{T}}^{\text {iso }}$ de fotones y electrones, en particular después de aplicar la corrección, dando confianza en la hipótesis de usar electrones para modelar los fotones.

Para estudiar el efecto en las distribuciones obtenidas de $Z \rightarrow e^{+} e^{-}$en distintas regiones cinemáticas, en la Figura 8.9, se presentan las distribuciones agregando un corte en $H_{\mathrm{T}}$ para eventos $Z \rightarrow e^{+} e^{-}$en muestras MC y datos. Como es de esperar, la energía de aislamiento es más alta y la distribución se hace más ancha con $H_{\mathrm{T}}$. Desafortunadamente, la escasa estadística impide el uso del corte en $H_{\mathrm{T}}$ de la SR. En cualquier caso, el efecto se considera como una posible fuente de incerteza sistemática.

\subsubsection{Modelo de fondo}

El modelo de fondo se obtuvo de los datos, en eventos que pasan todos los criterios de identificación salvo los criterios de identificación tight de fotones. La muestra de pseudo-fotones seleccionada de esta 

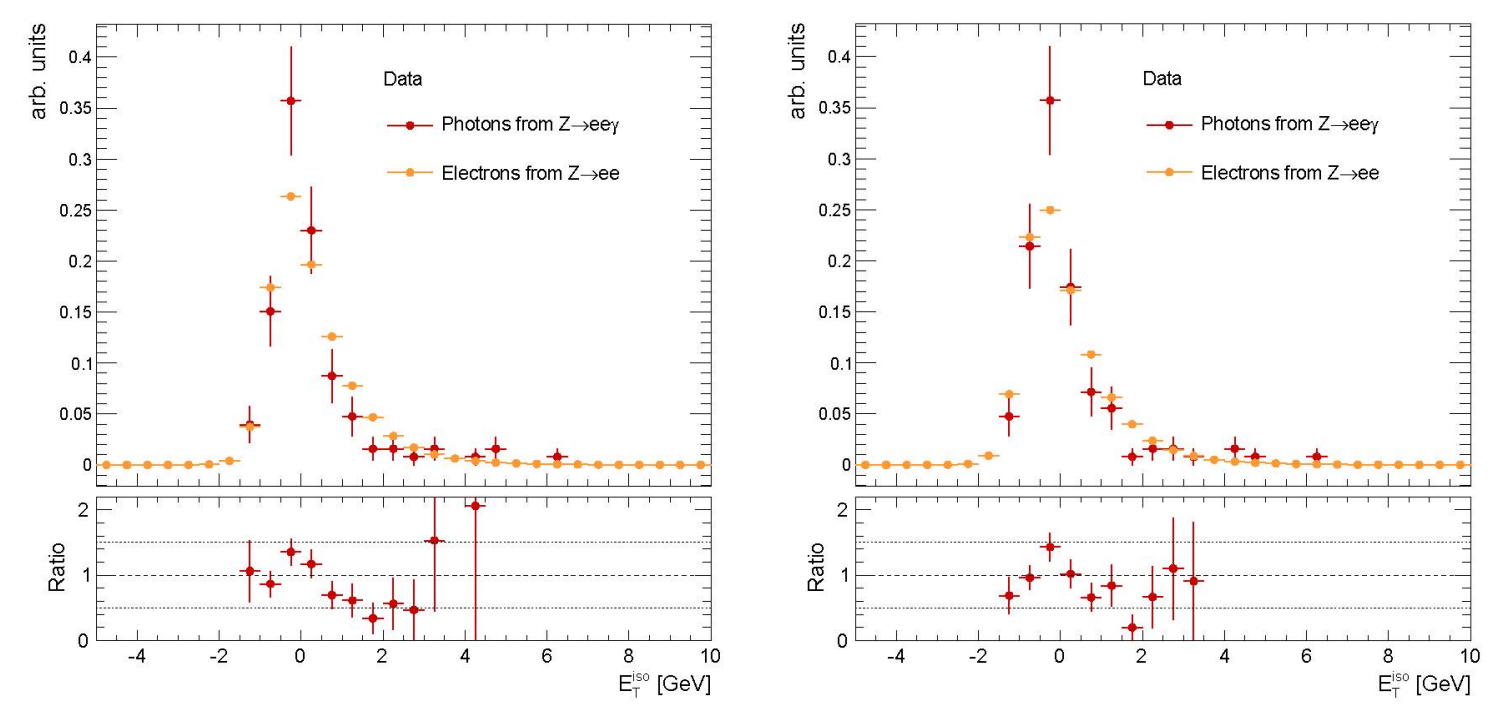

Figura 8.8: Comparación de las distribuciones de la energía de aislamiento para fotones reales de decaimiento radiativo del $Z$ y electrones provenientes del decaimiento $Z \rightarrow e^{+} e^{-}$antes (izquierda) y después (derecha) de la corrección por $p_{\mathrm{T}}$.
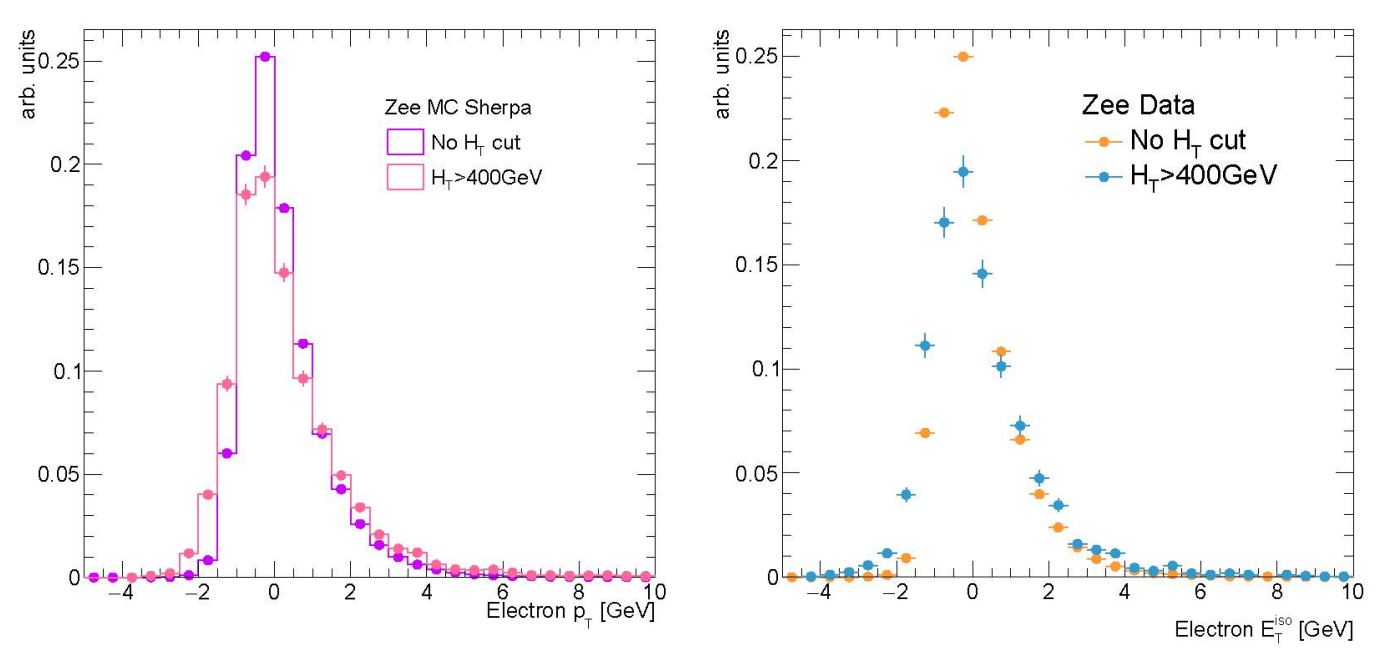

Figura 8.9: Dependencia de la energía de aislamiento de electrones con $H_{\mathrm{T}}$ para MC (izquierda) y datos (derecha) $Z \rightarrow e^{+} e^{-}$

forma debe presentar un perfil de aislamiento similar a la de fotones reales, permitiendo una pequeña contaminación de señal y alta estadística.

Se realizó un estudio detallado en muestras MC para encontrar el mejor conjunto de los cortes utilizados por el criterio de identificación tight que debía ser revertido para modelar de la mejor manera la distribución de aislamiento para los fotones falsos provenientes de decaimientos de hadrones en la SR. En la Figura 8.10 se puede ver la comparación de dos definiciones distintas de pseudo-fotones para MC y datos. Los pseudo-fotones loose-non-tight pasan los cortes de identificación loose, pero fallan al menos uno de los cortes tight. A pesar de la escasa estadística, resulta claro que falla en su objetivo de modelar el fondo tanto en datos como en MC. Se obtiene un mejor acuerdo para la definición loose'-non-tight, en la cual los fotones pasan todos los cortes tight salvo (al menos) uno de las variables en la sección de bandas del calorímetro $\left(F_{\text {side }}, w_{s 3}, E_{\text {ratio }}, \Delta E\right)$. Estas variables son construidas con solo algunas bandas centrales alrededor de la dirección del fotón, y por lo tanto se espera que no tengan una correlación con la energía de aislamiento del fotón, para cuyo cálculo se 
excluye la energía en las celdas centrales para tener en cuenta la propia energía del fotón. Utilizando esta definición, los pseudo-fotones tienen un perfil de aislamiento con una forma similar a la de fotones tight y por lo tanto es posible la extrapolación a la SR.
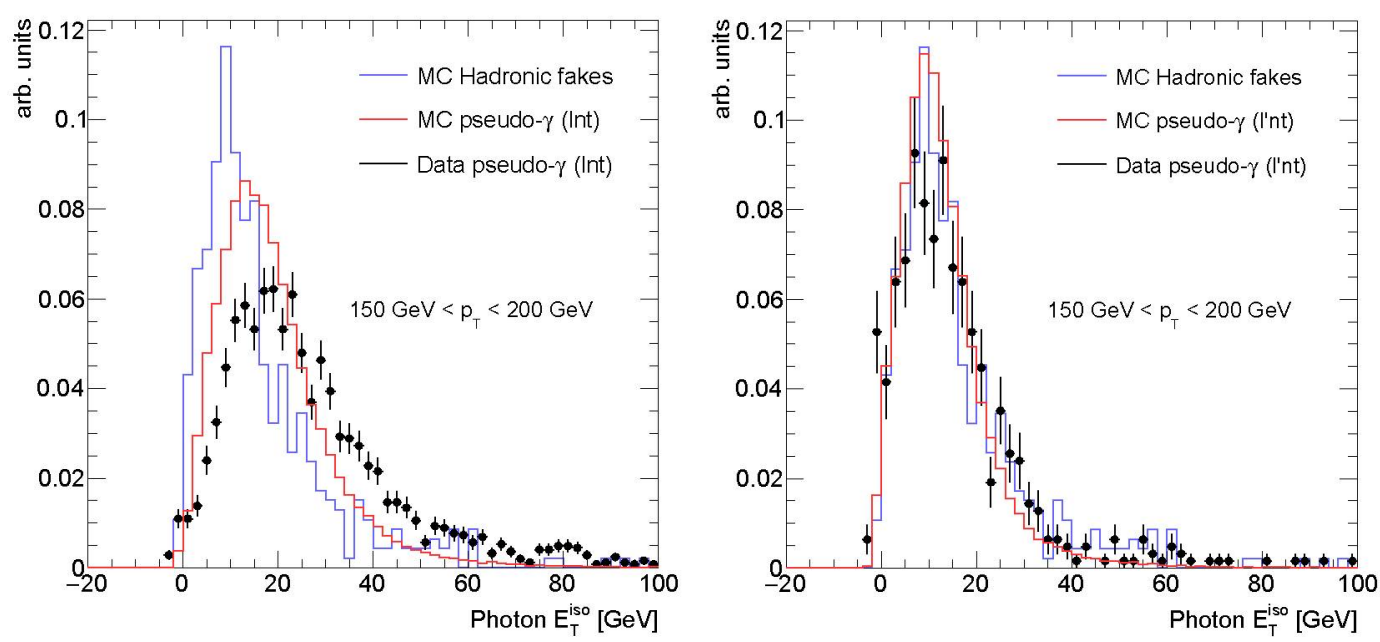

Figura 8.10: Distribución de la energía de aislamiento para fotones falsos provenientes de decaimientos hadrónicos (a nivel generador), comparada con la de pseudo-fotones obtenidos con dos definiciones diferentes: loose-non-tight (izquierda) y loose'-non-tight (derecha). Ver detalles en el texto.

Más aún, como se ve en la Figura 8.11, los fotones loose'-non-tight reproducen mejor el espectro de $p_{\mathrm{T}}$ de los fotones tight, y no es necesario aplicar ninguna corrección adicional.

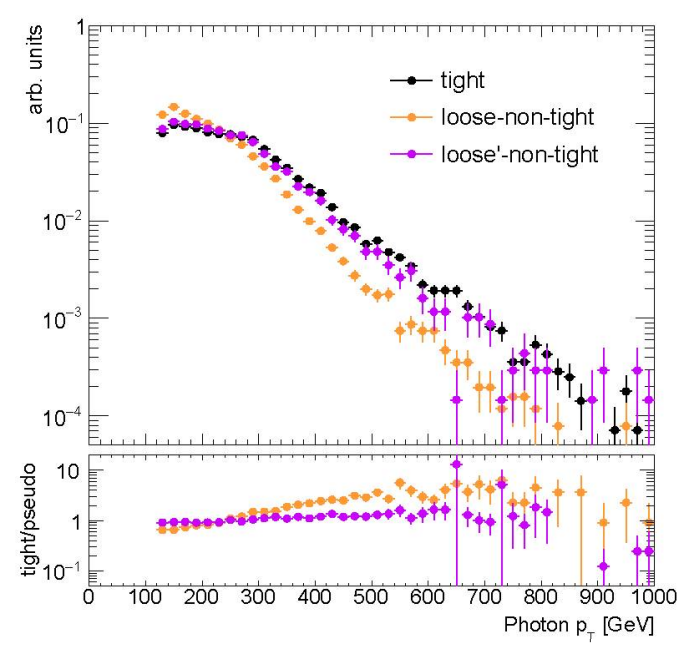

Figura 8.11: Distribución del $p_{\mathrm{T}}$ del fotón para candidatos tight y las dos definiciones diferentes de pseudo-fotones.

La distribución de la energía de aislamiento de los pseudo-fotones puede variar en distintas regiones cinemáticas. Es por esta razón que para derivar el modelo de fondo se utiliza una región relajada (CRJ), cercana a las regiones de señal, definida en la Tabla 8.7. Para mantener esta región ortogonal a las SR, 
se requiere un corte intermedio en $E_{\mathrm{T}}^{\text {miss }}$, y algunos otros cortes son relajados para ganar estadística. Como se espera, a mayor actividad hadrónica en el evento, mayor es la energía de aislamiento del fotón. En la Figura 8.12 se pueden ver las diferencias para distintos requerimientos en $H_{\mathrm{T}}$. En la región CRJ se utiliza un corte intermedio entre ambas SR, y las posibles diferencias son tenidas en cuenta como incerteza sistemática.

Tabla 8.7: Definición de la región utilizada para la estimación de los jets mal identificados como fotones.

\begin{tabular}{|c|c|}
\hline & CRJ \\
\hline$p_{\mathrm{T}}($ pseudo $-\gamma)[\mathrm{GeV}]>$ & 125 \\
\hline$N_{\text {leptones }}$ & 0 \\
\hline$E_{\mathrm{T}}^{\mathrm{miss}}[\mathrm{GeV}]$ & {$[50,150]$} \\
\hline$N_{\text {jets }} \geq$ & 2 \\
\hline$p_{\mathrm{T}}^{\text {jet }_{1}, \text { jet }_{2}}[\mathrm{GeV}]>$ & 100 \\
\hline$\Delta \phi\left(\right.$ jet,$\left.E_{\mathrm{T}}^{\text {miss }}\right)>$ & 0.4 \\
\hline$H_{\mathrm{T}}[\mathrm{GeV}]>$ & 600 \\
\hline
\end{tabular}
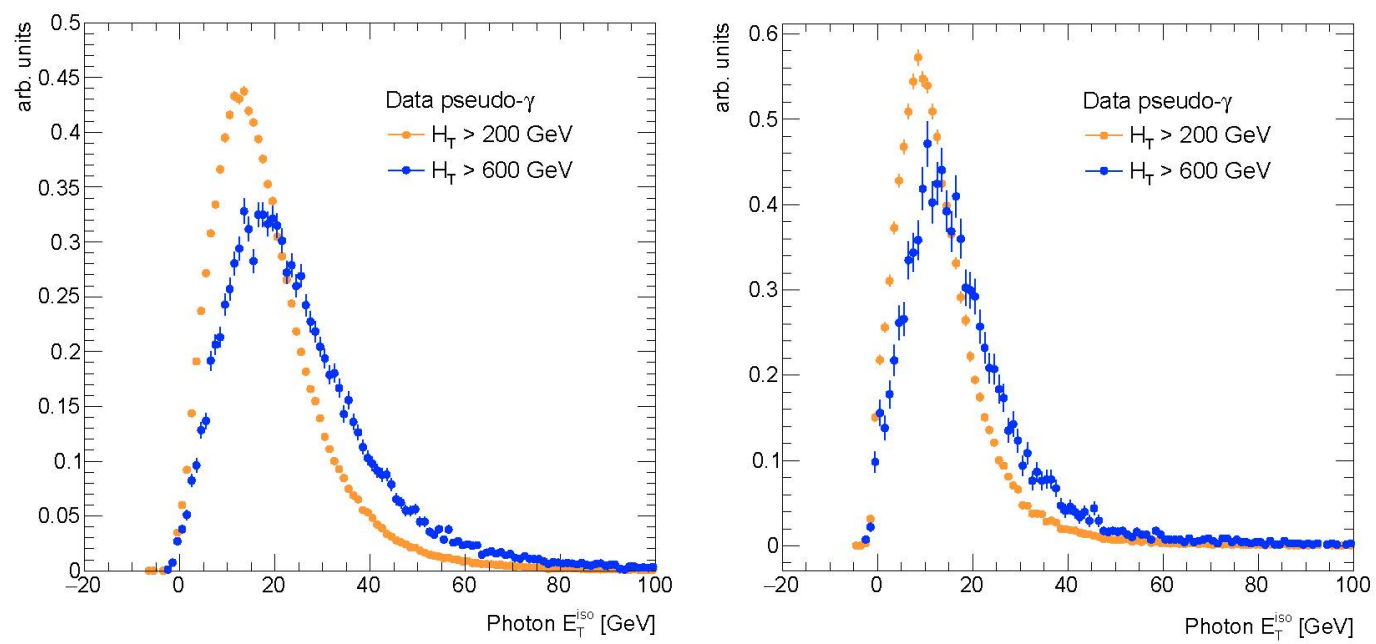

Figura 8.12: Distribución de la energía de aislamiento para pseudo-fotones loose-non-tight (izquierda) y loose'-non-tight (derecha), luego de la selección con un corte en $H_{\mathrm{T}}>200 \mathrm{GeV}$ y $H_{\mathrm{T}}>600 \mathrm{GeV}$.

Finalmente, se encuentra que la distribución de los pseudo-fotones está bien descripta por una función Crystall-Ball. La distribución en datos y el ajuste puede verse en la Figura 8.13.

\subsubsection{Ajuste combinado y estimación del fondo}

A fin de modelar la forma de la energía de aislamiento de los fotones tight en los datos, se utiliza el modelo de señal y fondo descriptos en las secciones anteriores, como se puede apreciar en la Figura 8.13. La distribución de $E_{\mathrm{T}}^{\text {iso }}$ para todos los eventos que pasan los criterios de selección de la región relajada CRJ es ajustada a una combinación lineal de los modelos de señal y fondo. El ajuste combinado y 
cada componente de la distribución pueden verse en Figura 8.13. Los parámetros son inicializados con los valores extraídos de los ajustes individuales a la señal y el fondo, y se los permite variar dentro de su incerteza

Para estimar la incerteza sistemática, el ajuste combinado es realizado sin ninguna limitación en los parámetros. La incerteza obtenida es del $50 \%$ del valor de $f_{j \rightarrow \gamma}$, lo suficientemente grande para contener cualquier potencial inexactitud en el modelado de la señal y el fondo.

El número de fotones total $\left(\mathrm{N}_{\text {total }}\right)$ y falsos $\left(\mathrm{N}_{j \rightarrow \gamma}\right)$ esperados es obtenido integrando las componentes de fondo y total del ajuste combinado sobre todo el rango $E_{\mathrm{T}}^{\text {iso }}<5 \mathrm{GeV}$, respectivamente:

$$
\begin{aligned}
& \mathrm{N}_{j \rightarrow \gamma}=24143 \pm 56 \\
& \mathrm{~N}_{\text {total }}=281813 \pm 586
\end{aligned}
$$

La fracción $f_{j \rightarrow \gamma}$ es obtenida simplemente como el cociente en la ecuación (8.3):

$$
f_{j \rightarrow \gamma}=0.0857 \pm 0.0003 \text { (stat.) } \pm 0.04 \text { (sist.) }
$$
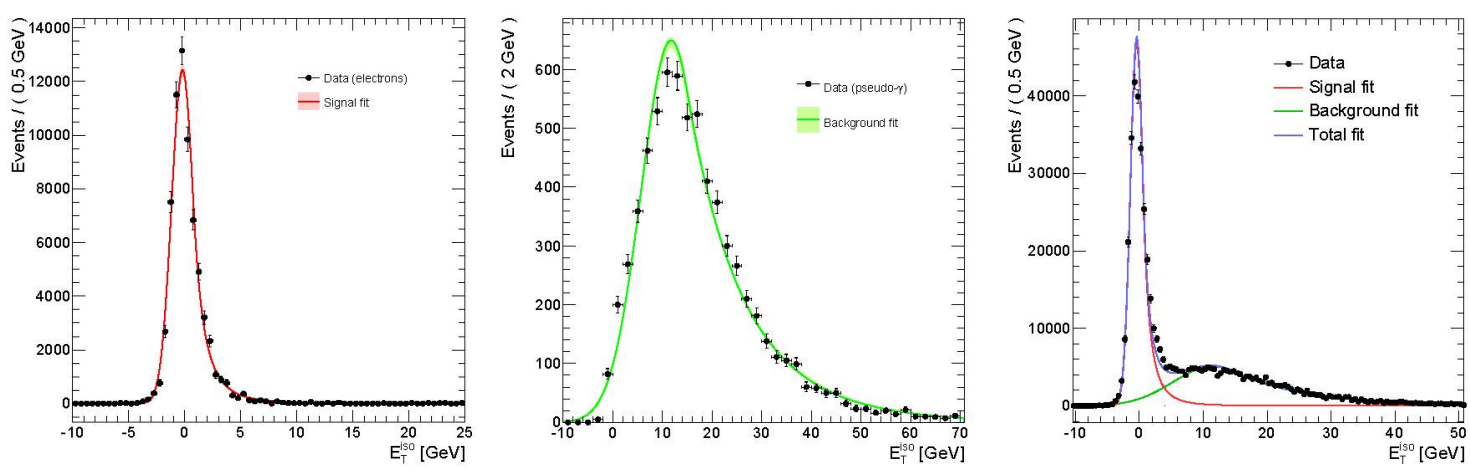

Figura 8.13: Ajuste a las distribuciones de $E_{\mathrm{T}}^{\mathrm{iso}}$ de señal (izquierda), fondo (centro) y combinado (derecha), en la región relajada CRJ.

El número de jets identificados erróneamente como fotones puede ser estimado utilizando la fracción $f_{j \rightarrow \gamma}$ como,

$$
N_{j \rightarrow \gamma}=f_{j \rightarrow \gamma} \cdot N_{\text {tight }}
$$

Para estimar la contribución en las SR, sin necesidad de usar el número de eventos en las SR, lo que implicaría utilizar los datos en la región de búsqueda, se parametrizó este número como función de $E_{\mathrm{T}}^{\mathrm{miss}}$, como se puede ver en Figura 8.14, utilizando una función exponencial $N_{j \rightarrow \gamma}=\exp \left(a+b E_{\mathrm{T}}^{\mathrm{miss}}\right)$ que luego se extrapola a la SR. Los valores de $a$ y $b$ obtenidos del ajuste se muestran en la Tabla 8.8.

Tabla 8.8: Parámetros que resultan del ajuste a la distribución de $N_{j \rightarrow \gamma}$ como función de $E_{\mathrm{T}}^{\text {miss }}$ con una función exponencial $\exp \left(a+b E_{\mathrm{T}}^{\mathrm{miss}}\right)$.

\begin{tabular}{crr}
\hline Parámetro & $\mathrm{SR}_{\mathrm{L}}$ & $\mathrm{SR}_{\mathrm{H}}$ \\
\hline $\mathrm{a}$ & $3.87 \pm 1.25$ & $1.80 \pm 0.98$ \\
$\mathrm{~b}$ & $-0.054 \pm 0.018$ & $-0.047 \pm 0.014$ \\
\hline
\end{tabular}


La contaminación esperada de fotones falsos en las SR se estima integrando la parametrización de $N_{j \rightarrow \gamma}$ sobre la región de $E_{\mathrm{T}}^{\text {miss }}$ de cada SR.

$$
\begin{aligned}
& N_{j \rightarrow \gamma}^{\mathrm{SR}_{\mathrm{L}}}=\int_{200}^{\infty} N_{j \rightarrow \gamma}\left(E_{\mathrm{T}}^{\mathrm{miss}}\right) d E_{\mathrm{T}}^{\mathrm{miss}}=0.01 \pm 0.02 \\
& N_{j \rightarrow \gamma}^{\mathrm{SR}_{\mathrm{H}}}=\int_{300}^{\infty} N_{j \rightarrow \gamma}\left(E_{\mathrm{T}}^{\mathrm{miss}}\right) d E_{\mathrm{T}}^{\text {miss }}=0.0001 \pm 0.0001
\end{aligned}
$$

Debido a que las incertezas son de igual o mayor magnitud que los valores centrales, se utilizaron los valores de estas como la estimación final en las SR.
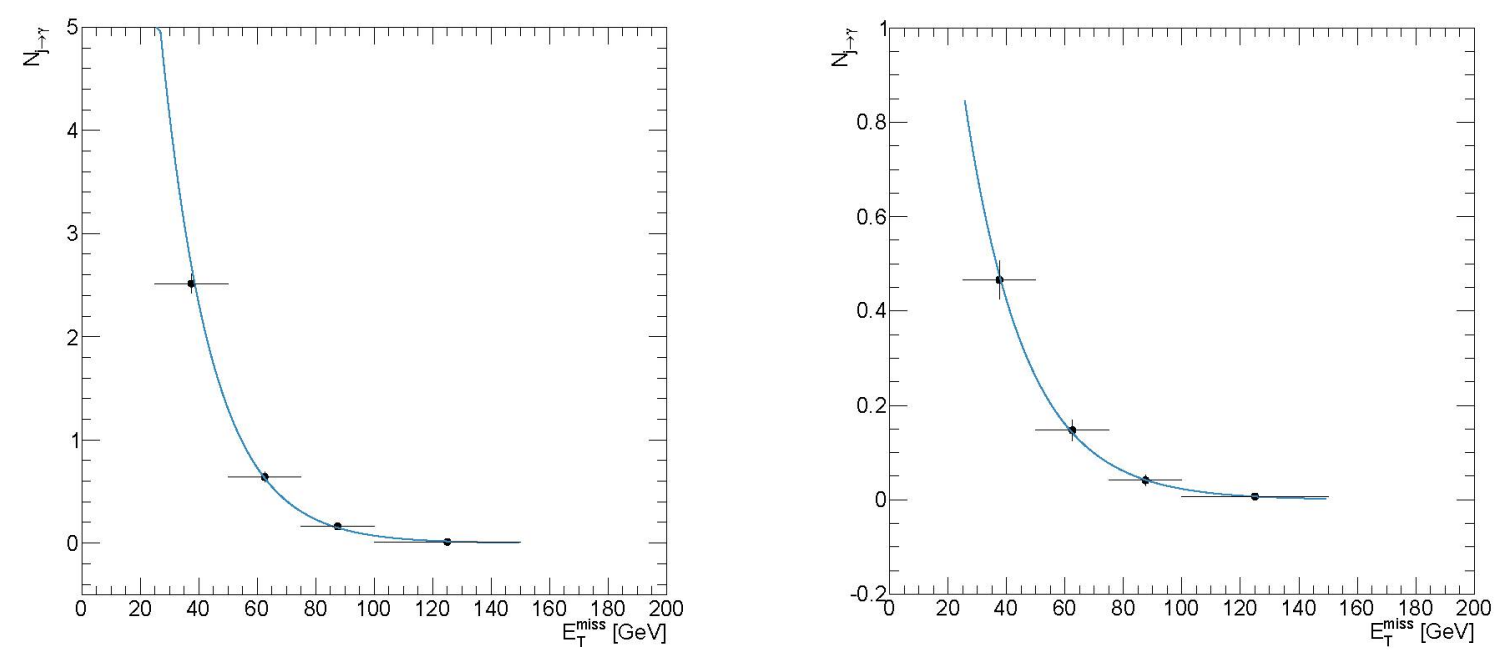

Figura 8.14: Parametrización del número de jets mal identificados como función de $E_{\mathrm{T}}^{\text {miss }}$ para $\mathrm{SR}_{\mathrm{L}}$ (izquierda) y $\mathrm{SR}_{\mathrm{H}}$ (derecha).

El fondo de jets identificados como fotones es estimado similarmente en las regiones de control y validación, multiplicando el número de eventos observado en cada caso por el factor $f_{j \rightarrow \gamma}$. 


\section{RESULTADOS E INTERPRETACIÓN}

Como se describió en el capítulo 5 , en el que se presentó la estrategia general para la búsqueda de nueva física realizada en esta tesis, a fin de obtener los resultados finales es necesario realizar un complejo análisis estadístico. Los detalles de este análisis se describen en la sección 9.1. Este se lleva a cabo utilizando las regiones de control para la normalización de los fondos, las regiones de validación y las regiones donde se espera la señal de nueva física, definidas en los capítulos anteriores. También deben ser incluidas las incertezas sistemáticas, tanto teóricas como experimentales, las cuales se presentan en la sección 9.2.

Los resultados del ajuste del likelihood se presentan en la sección 9.3 para las distintas regiones, para luego, de acuerdo a los resultados obtenidos, establecer los límites a los procesos de nueva física en la sección 9.4. Estos resultados son finalmente interpretados en el contexto de un modelo particular de supersimetría, el cual motiva el trabajo de esta tesis, en la sección 9.5.

Por último, en la sección 9.6, se muestra la significancia esperada para el análisis de los datos $\sqrt{s}=13 \mathrm{TeV}$ recolectados por ATLAS durante el año 2015 .

\subsection{Análisis estadístico}

A partir de la función likelihood general (ecuación (5.4)), se define la función likelihood utilizada en el análisis específico de esta tesis como:

$$
L\left(\boldsymbol{n} \mid \mu_{s}, \boldsymbol{\mu}_{p}, \boldsymbol{s}, \boldsymbol{b}, \boldsymbol{\alpha}\right)=\mathcal{P}_{\mathrm{SR}} \times \mathcal{P}_{\mathrm{CRQ}} \times \mathcal{P}_{\mathrm{CRW}} \times \mathcal{P}_{\mathrm{CRT}} \times \mathcal{C}_{\mathrm{syst}}
$$

donde $\boldsymbol{n}$ es el número de eventos observado en cada región (de control y señal) y $\mu_{s}$ es la intensidad de la señal que constituye el parámetro de interés del análisis. Los demás son parámetros nuisance. Por un lado, los parámetros de normalización de los fondos $\left(\boldsymbol{\mu}_{p}\right)$, en este caso $\mu_{Q}$ para el fondo de $\gamma+$ jets, $\mu_{W}$ para $W \gamma$ y $\mu_{T}$ para $t \bar{t} \gamma$ así como el número de eventos esperado de señal y fondo en cada región ( $\boldsymbol{s}$ y $\boldsymbol{b}$ respectivamente) que provienen de las muestras $\mathrm{MC}$ o de aquellos calculados a partir de los datos como se describió en el capítulo 8. Por otro lado, los parámetros $\alpha$ parametrizan las incertezas sistemáticas en la señal y el fondo utilizando una distribución normal.

Con la función likelihood, se construye el estadístico de prueba como el profile likelihood ratio,

$$
q_{\mu_{s}}=-2 \ln \left(\frac{L\left(\mu_{s}, \hat{\boldsymbol{\theta}}\right)}{L\left(\hat{\mu}_{s}, \hat{\boldsymbol{\theta}}\right)}\right)
$$

que será utilizado en este análisis para poner a prueba la compatibilidad de los datos observados con las predicciones del SM.

Realizando un ajuste simultáneo a los datos en las CR (o en las CR y SR), los parámetros descriptos 
anteriormente pueden ser estimados teniendo en cuenta apropiadamente sus correlaciones e incertezas. Además, como los parámetros de normalización de los fondos $\left(\boldsymbol{\mu}_{p}\right)$ dependen esencialmente de las regiones de control con una alta estadística de datos (respecto a la SR), se reduce el impacto de estas incertezas estadísticas en las SR.

El análisis estadístico se implementó utilizando HistFitTer [97], una herramienta desarrollada por el grupo de SUSY de ATLAS, la cual utiliza las librerías RooFit, RooStats [151] y HistFaCtory [96]. El hecho de utilizar una misma herramienta en distintos análisis dentro de la colaboración, permite que su combinación estadística sea más sencilla.

\subsection{Incertezas sistemáticas}

Uno de los aspectos más importantes de las investigaciones con datos recolectados por ATLAS es la determinación de todas las incertezas sistemáticas que afectan a los resultados. Las incertezas sistemáticas están asociadas a las predicciones de todas las componentes del fondo y a los valores esperados de señal. Estas incertezas pueden ser clasificadas en dos tipos: de origen experimental o teórico, y pueden tener un impacto importante en el número de eventos esperado en las CR y SR como así también en los parámetros $\mu_{p}$ utilizados en la extrapolación del fondo. En esta sección se presentan todas las fuentes posibles de incertezas sistemáticas incluidas en el presente análisis.

Cabe mencionarse que, en la determinación de los resultados finales utilizando el ajuste con el PLR, las predicciones de los fondos se permiten variar dentro del tamaño de las incertezas sistemáticas y por lo tanto son restringidas por los datos.

\subsubsection{Incertezas experimentales}

A continuación se describen los procedimientos realizados para evaluar las incertezas experimentales comunes para todos los procesos tanto de señal como de fondo considerados en el análisis.

\section{Luminosidad}

Existen dos detectores diseñados específicamente para la medida de la luminosidad en ATLAS: LUCID y BCM [152]. La luminosidad es calculada midiendo la tasa de interacción durante la toma de datos mediante estos dispositivos dispuestos a cada lado de ATLAS, a pequeños ángulos respecto de la dirección del haz. La calibración absoluta se ha determinado a partir de los parámetros del acelerador mediante una serie de estudios dedicados, conocidos como Van der Meer scans [153], realizados durante el mes de Noviembre del año 2012. La incerteza sistemática relativa en la medida de la luminosidad integrada estimada es $\pm 2.8 \%$ [69].

Esta incerteza debe ser propagada a todas las muestras simuladas utilizadas para la estimación de fondo y señal, las que deben ser normalizadas a la luminosidad de datos analizada.

\section{Trigger}

La estimación de la eficiencia del trigger de fotones utilizado y su incerteza se presentó en detalle en la sección 7.2. La incerteza sistemática resulta $<1 \%$.

\section{Pile-up}

En la sección 6.1.3 se presentó la necesidad de realizar un repesado evento a evento en las muestras simuladas para modelar las condiciones de pile-up de los datos, ya que las muestras MC se simulan 
con un perfil de pile-up fijo. Para calcular la incerteza asociada a este procedimiento se varía el valor medio del número de interacciones por cruce de bunches en $\pm 10 \%$.

\section{Escala de energía y resolución de energía de fotones y leptones}

Como se menciona en la sección 3.2.2, es necesario aplicar factores de escala a las muestras MC para corregir la eficiencia de identificación de electrones y fotones. Estos factores son provistos por los grupos de Egamma de ATLAS, y la incerteza en estos factores, la cual es menor al 3\%, es propagada a través del análisis, siguiendo [154-157].

La estimación de la incerteza en la escala y resolución de energía de los fotones y electrones [156] también es considerada y posee el mismo orden de magnitud.

Como el requerimiento de aislamiento de fotones aplicado por la herramienta oficial para derivar los factores de corrección es distinto al utilizado en este análisis, la eficiencia de identificación fue evaluada nuevamente para los distintos criterios de aislamiento para fotones con $p_{\mathrm{T}}>125 \mathrm{GeV}$ en muestras MC de señal y fondo. Se encontró que el efecto es $<1 \%$ y por lo tanto es despreciado.

\section{Escala y resolución de energía de los jets}

La calibración de la escala de energía de los jets a la escala hadrónica correspondiente, tiene una incerteza asociada debida a distintas fuentes, entre las que se encuentran la respuesta del calorímetro y el pile-up. La sobre-estimación o subestimación de la energía puede resultar en que se seleccione un conjunto distinto de jets para el análisis, debido a que la corrección no es la misma para todos los jets y depende de sus propiedades. Para evaluar el efecto de esta incerteza en los eventos seleccionados, la corrección es modificada en $\pm 1 \sigma$, utilizando una herramienta provista por el grupo de JET/ETMISS de ATLAS [158].

El impacto de la incerteza asociada a la resolución de energía de los jets es considerado comparando el número de eventos obtenido utilizando los jets nominales seleccionados para el análisis con el obtenido modificando la energía de los jets de acuerdo a una distribución gaussiana, de media 1 y ancho igual a la incerteza.

\section{Eficiencia de identificación de $b$-jets}

La identificación de b-jets solo es utilizada en la selección de las regiones de control CRW y CRT. En estas regiones, se evaluó la incerteza variando los factores de escala aplicados a cada jet en las simulaciones, que dependen de $\eta$ y $p_{\mathrm{T}}$, dentro del rango que refleja la incerteza sistemática en la eficiencia medida de identificación de $b$-jets. Esta eficiencia fue medida en datos y para el algoritmo utilizado MV1 (y la configuración que se corresponde a una eficiencia de 70\% para una muestra $t \bar{t}$ ), la incerteza varía entre $5 \%$ y $19 \%$, aumentando con el $p_{\mathrm{T}}$ del jet [88].

\section{Energía faltante}

Al modificar la escala de energía de los objetos o la resolución de energía de los jets, el efecto se propaga al término correspondiente en el cálculo de $E_{\mathrm{T}}^{\text {miss }}$. Adicionalmente, también se evalúa la incerteza en el término soft de $E_{\mathrm{T}}^{\text {miss }}$, variando la escala y resolución de energía de los clusters, utilizando una herramienta provista por el grupo JeT/ETMISS de ATLAS.

\section{Estimación de los fondos de electrones y jets mal identificados}

La estimación de los fondos provenientes de la mala identificación de electrones o jets a partir de los datos ha sido discutida en detalle en las secciones 8.3 y 8.4 . 
En el caso de electrones dicha estimación se obtiene multiplicando el número de eventos de una muestra de electrones en cada región de señal por el factor $f_{e \rightarrow \gamma}$ relacionado con la probabilidad de que un electrón sea identificado como un fotón. Este factor es calculado a partir de una muestra de datos $Z \rightarrow e^{+} e^{-}$y varía entre $1.4 \%$ y $3.3 \%$ dependiendo del $|\eta|$ del electrón, con una incerteza sistemática del $25 \%-35 \%$. El efecto de la incerteza sistemática en el factor $f_{e \rightarrow \gamma}$ se propaga en el análisis comparando el número de eventos obtenido en las SR, modificando este factor en $\pm 1 \sigma$.

El fondo de jets identificados como fotones es estimado teniendo en cuenta las diferencias en el perfil de la energía de aislamiento entre fotones reales y falsos. A partir de estas diferencias se calcula la fracción de fotones falsos $f_{j \rightarrow \gamma}=0.0857 \pm 0.0002$ (stat.) \pm 0.04 (sist.). La incerteza sistemática en esta fracción tiene en cuenta las posibles incertezas del método utilizado, y se propaga al número de eventos en las $\mathrm{SR}$, variando este factor en $\pm 1 \sigma$.

\subsubsection{Incertezas teóricas}

\section{Señal de SUSY}

La sección eficaz de producción de pares de gluinos fue calculada utilizando el programa NLL-FAST, al igual que las incertezas asociadas debido a la elección de la escala de renormalización y factorización, y a la elección de la PDF. Para ello se siguen las recomendaciones PDF4LHC [127] como se describe en detalle en la sección 6.2.2.

Los valores centrales y sus incertezas para las diferentes masas de gluinos consideradas se resumen en la Tabla 9.1. Las incertezas totales varían entre $22.5 \%$ para $m_{\widetilde{g}}=800 \mathrm{GeV}$ y $36.8 \%$ para $m_{\widetilde{g}}=1.3 \mathrm{TeV}$.

\section{Producción de $t \bar{t} \gamma$}

La incerteza teórica asociada a la elección de la configuración utilizada en la simulación MC es tenida en cuenta considerando variaciones en la generación de eventos con respecto a la configuración nominal. Las variaciones consideradas incluyen las escalas de factorización y renormalización $(0.5 \times, 2 \times)$, $\alpha_{s}$, y el modelo ISR/FSR.

La Tabla 9.2 resume los cambios relativos en el número de eventos esperado en cada región de señal para cada una de las variaciones con respecto a la muestra nominal como resultado de un estudio realizado a nivel generador. La diferencia principal entre todas las variaciones y la configuración nominal aparece mayormente en los cortes en $H_{\mathrm{T}}$ y $R_{\mathrm{T}}^{4}$, como es esperado ya que las propiedades de la actividad hadrónica del evento son las más sensibles a las variaciones de escala.

La incerteza de cada una de las variaciones es simetrizada y luego se suman en cuadratura para obtener la incerteza teórica total, que resulta en $56 \%$ y $28 \%$ para $\mathrm{SR}_{\mathrm{L}}$ y $\mathrm{SR}_{\mathrm{H}}$, respectivamente.

\section{Producción de $W \gamma$}

De manera similar a lo realizado para estimar las incertezas teóricas de la producción de $t \bar{t} \gamma$, las incertezas en el número estimado de eventos de $W \gamma$ fueron determinadas realizando el análisis a nivel generador para las muestras simuladas con variaciones de la configuración nominal. Para las incertezas debidas a la elección de escala de factorización/renormalización, se simularon muestras modificando la escala al doble y la mitad. También se consideró la incerteza debida a la escala de asociación entre ME y PS, cuyo valor nominal es de $20 \mathrm{GeV}$, y para el caso de las variaciones se modificó a 15 y $30 \mathrm{GeV}$.

Los valores obtenidos a partir de las diferentes fuentes de incertezas sistemáticas se resumen en la Tabla 9.3, donde el valor total es de $73 \%$ y $39 \%$ para $\mathrm{SR}_{\mathrm{L}}$ y $\mathrm{SR}_{\mathrm{H}}$, respectivamente. 


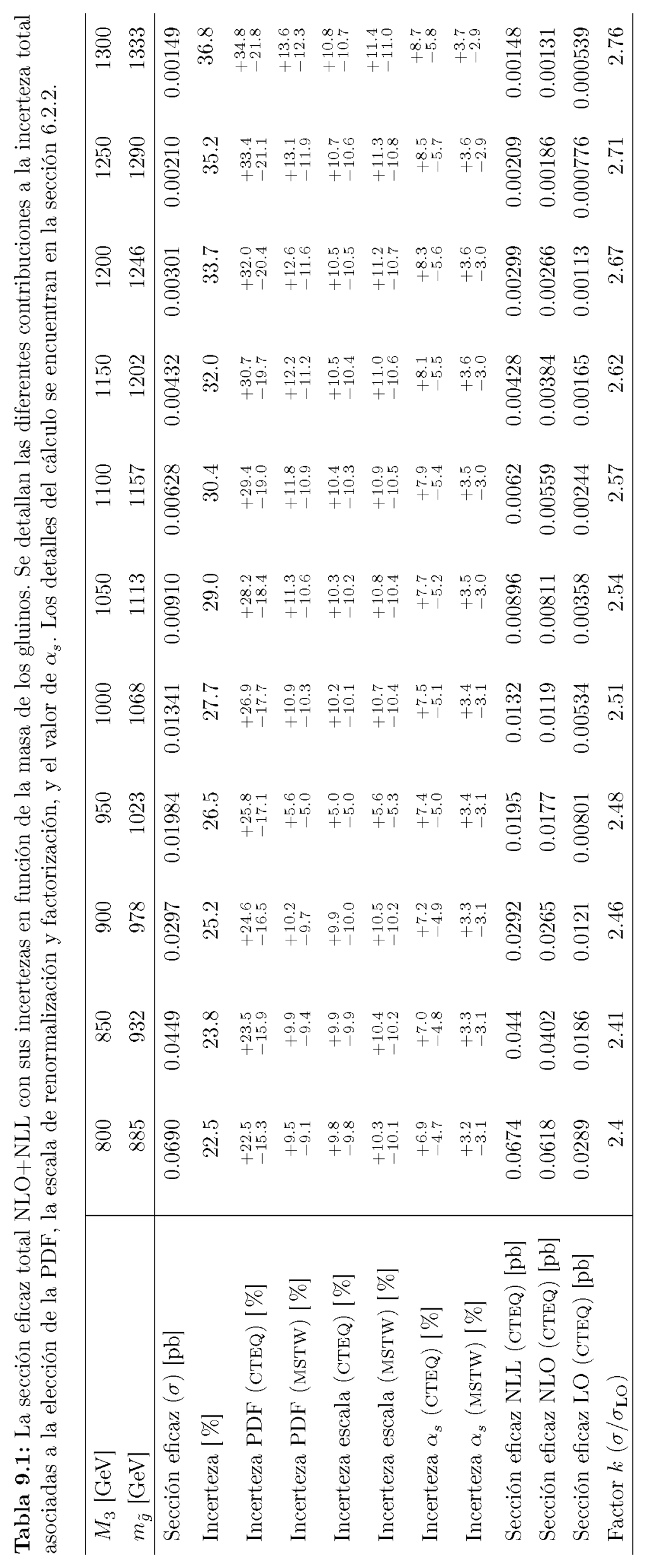


Tabla 9.2: Resumen de las incertezas teóricas de la producción de $t \bar{t} \gamma$ obtenidas en cada región de señal.

\begin{tabular}{lrr}
\hline & $\mathrm{SR}_{\mathrm{L}}$ & $\mathrm{SR}_{\mathrm{H}}$ \\
\hline Escala de factorización/renormalización & $56 \%$ & $20 \%$ \\
ISR/FSR & $4 \%$ & $20 \%$ \\
$\alpha_{s}$ & $4 \%$ & $0 \%$ \\
\hline Total & $56 \%$ & $28 \%$ \\
\hline
\end{tabular}

Tabla 9.3: Resumen de las incertezas teóricas de la producción de $W \gamma$ obtenidas en cada región de señal.

\begin{tabular}{lcc}
\hline & $\mathrm{SR}_{\mathrm{L}}$ & $\mathrm{SR}_{\mathrm{H}}$ \\
\hline Escala de factorización & $0 \%$ & $16 \%$ \\
Escala de renormalización & $71 \%$ & $33 \%$ \\
Escala de asociación ME/PS (CKKW) & $14 \%$ & $12 \%$ \\
\hline Total & $73 \%$ & $39 \%$ \\
\hline
\end{tabular}

\section{Producción de fotones directos}

La normalización del fondo de $\gamma+$ jets y sus incertezas son tomadas del ajuste a datos en la CR como se describe en sección 8.2. Una incerteza adicional es obtenida comparando el número de eventos en las distintas regiones de señal entre la muestra nominal (SHERPA) y los obtenidos con una muestra generada con otro generador (PYTHIA). El estudio se realizó a nivel generador para evitar tener en cuenta nuevamente los efectos del detector. Esta incerteza resultó del $45 \%$.

\section{Otros fondos $\mathrm{MC}$}

La mayor contribución de procesos de fondo que se estiman directamente de las simulaciones MC en las regiones de señal proviene del $Z \gamma$, para el que se utilizó una incerteza conservativa del $100 \%$.

\subsection{Ajuste simultáneo en las regiones de control}

Para validar los métodos utilizados en la estimación de los fondos, se realiza un ajuste simultáneo utilizando únicamente las CR. El objetivo de este ajuste es estimar el fondo total esperado en las VR y las SR, sin hacer ninguna suposición del modelo de señal. Solo las muestras de fondo son incorporadas al modelo, y las CR se suponen libres de contaminación de señal. Cabe destacarse que el ajuste se realiza solamente en las CR, y los procesos de fondo dominantes son normalizados al número de eventos observado en cada una de las regiones. La función likelihood utilizada es la de la ecuación (9.1) sin el término de la SR. Como los parámetros de la pdf correspondientes al fondo son compartidos entre las diferentes regiones, el resultado del ajuste puede ser usado luego, para predecir el número de eventos en las VR y SR.

Las predicciones a partir del ajuste en las CR son independientes del número de eventos observado en cada SR y VR, lo que permite una comparación no sesgada entre el número de eventos predicho y observado en cada región. Los resultados de este ajuste extrapolados a las SR, también son importantes para que grupos externos al experimento puedan hacer una prueba de hipótesis en un modelo de nueva física no considerado por ninguno de los experimentos del LHC.

En las siguientes secciones se muestran los resultados obtenidos a partir de este ajuste en las distintas regiones del análisis. 


\subsubsection{Resultados en las regiones de control}

El número de eventos predicho para cada proceso de fondo antes y después del ajuste en las regiones de control puede verse en Tablas 9.4 y 9.5, comparado con el número de eventos observado. Estas regiones no solo determinan la normalización de los procesos de fondo dominante en cada una, sino que también restringen las incertezas sistemáticas aplicadas a todos los fondos. Es importante observar que las variables relevantes del análisis se encuentren bien modeladas en las regiones de control, lo cual puede observarse en las Figuras 9.2 y 9.3 .

En la Tabla 9.6 se presentan los parámetros de normalización de los fondos $W \gamma\left(\mu_{W}\right), t \bar{t} \gamma\left(\mu_{T}\right)$ y $\gamma+$ jets $\left(\mu_{Q}\right)$, obtenidos del ajuste realizado en las regiones de control. En su mayoría, los factores resultan consistentes con la unidad dentro de sus incertezas.

En la Figura 9.1 se encuentran resumidos todos los parámetros del ajuste simultáneo (las incertezas sistemáticas y los factores de normalización de los fondos). Los valores de los puntos representan los pulls en los parámetros nuisance, lo que indica el apartamiento de los valores centrales luego del ajuste. Las líneas de error representan el grado de disminución de las incertezas por el ajuste. Si este es muy grande puede resultar en un aumento artificial en la sensibilidad a nueva física, reduciendo las incertezas sistemáticas. Por este motivo resulta importante chequear el comportamiento de los parámetros nuisance después del ajuste. En este caso se observa que todos los parámetros dan resultados razonables y el valor central es prácticamente cero.

Los puntos de la derecha de la Figura 9.1, después de la línea punteada, representan los parámetros de normalización de los distintos fondos. Se puede ver que todos los valores centrales están contenidos dentro de una desviación estándar.
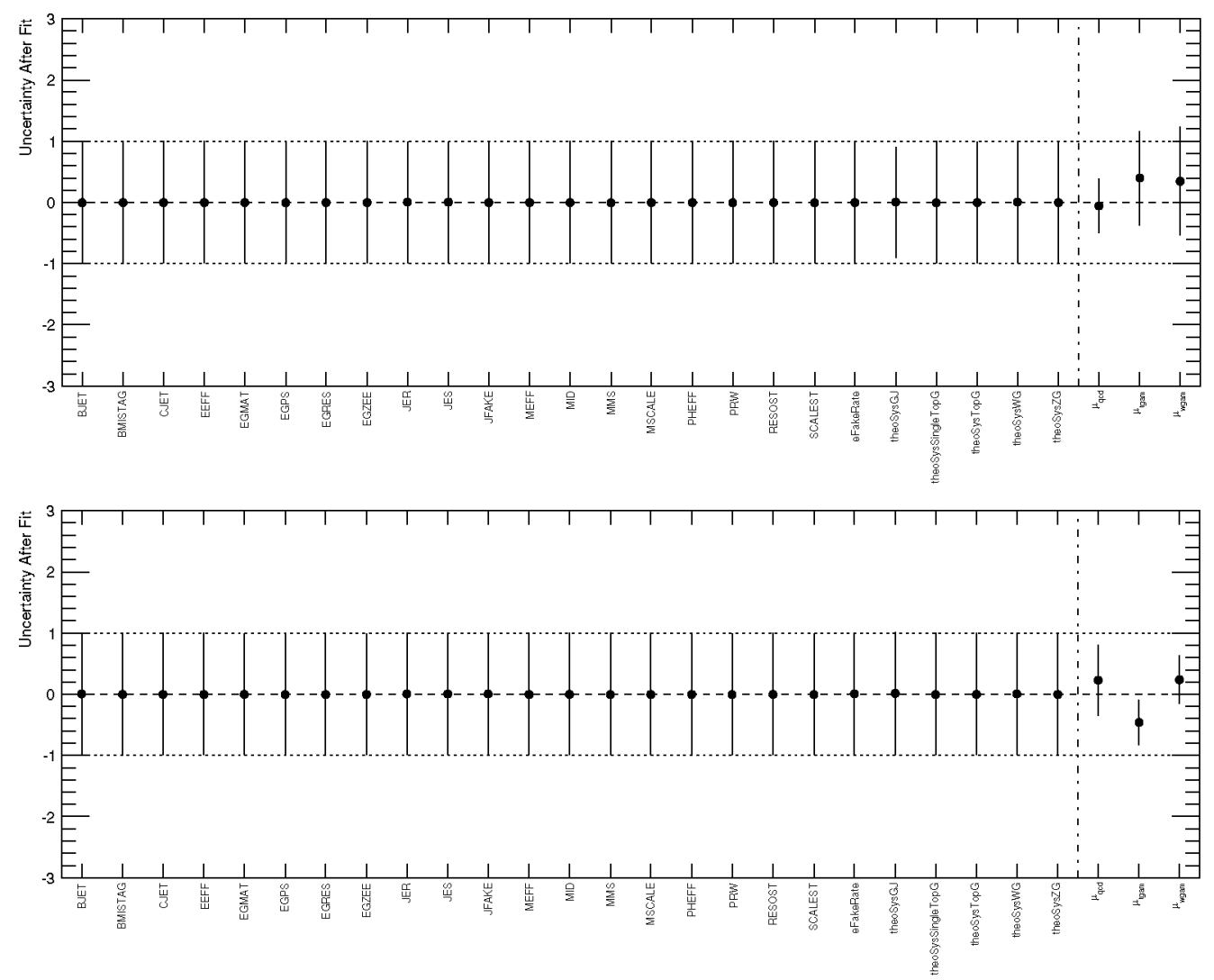

Figura 9.1: Resumen de los parámetros después del ajuste para $\mathrm{SR}_{\mathrm{L}}$ (arriba) y $\mathrm{SR}_{\mathrm{H}}$ (abajo). 
Tabla 9.4: Resultados del ajuste en las CR correspondientes a $\mathrm{SR}_{\mathrm{L}}$. El número de eventos observado es comparado con el número de eventos esperado de fondo, después de la correspondiente normalización en las CR. En la parte inferior de la tabla se muestran también lo valores nominales del fondo antes de la correspondiente normalización. Las incertezas incluyen la incerteza estadística y sistemática.

\begin{tabular}{|c|c|c|c|c|}
\hline & Regiones de control & $\mathrm{CRQ}_{\mathrm{L}}$ & $\mathrm{CRW}_{\mathrm{L}}$ & $\mathrm{CRT}_{\mathrm{L}}$ \\
\hline & Eventos observados & 1348 & 8 & 18 \\
\hline \multirow{11}{*}{$\begin{array}{l}\stackrel{\mathscr{B}}{\Xi} \\
\stackrel{\Xi}{0} \\
\stackrel{0}{0} \\
\stackrel{0}{0}\end{array}$} & Eventos esperados SM & $1347.21 \pm 36.51$ & $8.00 \pm 2.75$ & $18.00 \pm 4.16$ \\
\hline & $W \gamma$ & $0.28 \pm 0.27$ & $5.24 \pm 3.07$ & $3.15 \pm 1.94$ \\
\hline & $t \bar{t} \gamma$ & $2.22 \pm 1.31$ & $1.33 \pm 0.73$ & $10.66 \pm 4.92$ \\
\hline & $t \bar{t} \gamma(\mathrm{had})$ & $28.46 \pm 16.90$ & $0.00 \pm 0.00$ & $0.00 \pm 0.00$ \\
\hline & $V(\rightarrow q q) \gamma$ & $39.30 \pm 24.90$ & $0.00 \pm 0.00$ & $0.00 \pm 0.00$ \\
\hline & $t \gamma$ & $0.24 \pm 0.04$ & $0.22 \pm 0.06$ & $1.24 \pm 0.24$ \\
\hline & $Z(\rightarrow l l) \gamma$ & $0.08_{-0.08}^{+0.10}$ & $0.14_{-0.14}^{+0.14}$ & $0.11_{-0.11}^{+0.11}$ \\
\hline & $Z(\rightarrow \nu \nu) \gamma$ & $0.00 \pm 0.00$ & $0.00 \pm 0.00$ & $0.00 \pm 0.00$ \\
\hline & $\gamma+$ jets & $1155.79 \pm 68.55$ & $0.13 \pm 0.04$ & $0.01 \pm 0.01$ \\
\hline & $e \rightarrow \gamma$ & $5.34 \pm 0.89$ & $0.26 \pm 0.05$ & $1.29 \pm 0.21$ \\
\hline & $j \rightarrow \gamma$ & $115.48 \pm 54.49$ & $0.69 \pm 0.32$ & $1.54 \pm 0.72$ \\
\hline \multirow{11}{*}{$\frac{\mathscr{D}}{\stackrel{D}{Z}}$} & Eventos esperados SM & 1397.30 & 6.29 & 14.14 \\
\hline & $W \gamma$ & 0.21 & 3.90 & 2.34 \\
\hline & $t \bar{t} \gamma$ & 1.59 & 0.95 & 7.61 \\
\hline & $t \bar{t} \gamma(\mathrm{had})$ & 20.31 & 0.00 & 0.00 \\
\hline & $V(\rightarrow q q) \gamma$ & 29.25 & 0.00 & 0.00 \\
\hline & $t \gamma$ & 0.24 & 0.22 & 1.24 \\
\hline & $Z(\rightarrow l l) \gamma$ & 0.08 & 0.14 & 0.11 \\
\hline & $Z(\rightarrow \nu \nu) \gamma$ & 0.00 & 0.00 & 0.00 \\
\hline & $\gamma+$ jets & 1224.79 & 0.14 & 0.01 \\
\hline & $e \rightarrow \gamma$ & 5.34 & 0.26 & 1.29 \\
\hline & $j \rightarrow \gamma$ & 115.48 & 0.69 & 1.54 \\
\hline
\end{tabular}

\subsubsection{Resultados en las regiones de validación}

Los factores de normalización calculados a partir del ajuste simultáneo en las CR, y detallados en la Tabla 9.6, son utilizados para estimar los fondos en las VR, donde se compara el fondo esperado con los datos observados, a fin de validar la extrapolación que luego se hará a la SR.

En las Tablas 9.7 y 9.8 se presentan los resultados para las distintas regiones de validación asociadas a $\mathrm{SR}_{\mathrm{L}}$ y $\mathrm{SR}_{\mathrm{H}}$, respectivamente, y en la Figura 9.4 pueden verse las distribuciones de algunos observables en algunas de estas regiones. En general, el número de eventos de fondo predicho está en buen acuerdo con el número de eventos observado dentro de las incertezas, lo que permite validar el método empleado y la viabilidad de utilizar la estimación del número de eventos de fondo en las SR. Este resultado puede visualizarse más fácilmente en la Figura 9.6 donde se presentan los pull para las distintas regiones, definido como $\frac{n_{\text {obs }}-n_{\exp }}{\sigma_{\text {tot }}}$, donde $\sigma_{\text {tot }}$ es la suma de la incerteza estadística y sistemática. El mismo acuerdo puede verse de las distribuciones de los observables en las VR, que confirman que no existen problemas en el modelado de los distintos observables.

Habiendo validado el método de obtención del fondo contaminante en la región donde se espera la señal de nueva física, se procedió a la utilización de los datos experimentales en las denominadas regiones de señal, cuyos resultados pueden verse en la siguiente sección. 
Tabla 9.5: Resultados del ajuste en las CR correspondientes a $\mathrm{SR}_{\mathrm{H}}$. El número de eventos observado es comparado con el número de eventos esperado de fondo, después de la correspondiente normalización en las CR. En la parte inferior de la tabla se muestran también lo valores nominales del fondo antes de la correspondiente normalización. Las incertezas incluyen la incerteza estadística y sistemática.

\begin{tabular}{|c|c|c|c|c|}
\hline & Regiones de control & $\mathrm{CRQ}_{\mathrm{H}}$ & $\mathrm{CRW}_{\mathrm{H}}$ & $\mathrm{CRT}_{\mathrm{H}}$ \\
\hline & Eventos observados & 216 & 25 & 17 \\
\hline \multirow{11}{*}{$\stackrel{\mathscr{B}}{\stackrel{\mathscr{B}}{\mathscr{D}}}$} & Eventos esperados SM & $216.03 \pm 14.53$ & $25.00 \pm 4.99$ & $17.00 \pm 4.08$ \\
\hline & $W \gamma$ & $0.10 \pm 0.08$ & $19.32 \pm 5.62$ & $4.66 \pm 1.43$ \\
\hline & $t \bar{t} \gamma$ & $0.04 \pm 0.04$ & $1.91 \pm 1.34$ & $6.88 \pm 4.63$ \\
\hline & $t \bar{t} \gamma(\mathrm{had})$ & $0.31 \pm 0.24$ & $0.00 \pm 0.00$ & $0.00 \pm 0.00$ \\
\hline & $V(\rightarrow q q) \gamma$ & $3.46 \pm 1.53$ & $0.00 \pm 0.00$ & $0.00 \pm 0.00$ \\
\hline & $t \gamma$ & $0.01_{-0.01}^{+0.02}$ & $0.54 \pm 0.06$ & $1.51 \pm 0.18$ \\
\hline & $Z(\rightarrow l l) \gamma$ & $0.00 \pm 0.00$ & $0.57 \pm 0.57$ & $0.19_{-0.19}^{+0.19}$ \\
\hline & $Z(\rightarrow \nu \nu) \gamma$ & $0.00 \pm 0.00$ & $0.00 \pm 0.00$ & $0.00 \pm 0.00$ \\
\hline & $\gamma+$ jets & $193.31 \pm 16.91$ & $0.02_{-0.02}^{+0.94}$ & $0.00 \pm 0.00$ \\
\hline & $e \rightarrow \gamma$ & $0.27 \pm 0.04$ & $0.49 \pm 0.08$ & $2.31 \pm 0.37$ \\
\hline & $j \rightarrow \gamma$ & $18.52 \pm 8.70$ & $2.14 \pm 0.99$ & $1.46 \pm 0.68$ \\
\hline \multirow{11}{*}{ 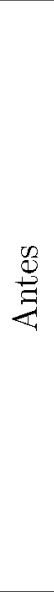 } & Eventos esperados SM & 179.24 & 22.94 & 22.04 \\
\hline & $W \gamma$ & 0.08 & 15.62 & 3.76 \\
\hline & $t \bar{t} \gamma$ & 0.08 & 3.57 & 12.82 \\
\hline & $t \bar{t} \gamma(\mathrm{had})$ & 0.58 & 0.00 & 0.00 \\
\hline & $V(\rightarrow q q) \gamma$ & 2.80 & 0.00 & 0.00 \\
\hline & $t \gamma$ & 0.01 & 0.54 & 1.51 \\
\hline & $Z(\rightarrow l l) \gamma$ & 0.00 & 0.57 & 0.19 \\
\hline & $Z(\rightarrow \nu \nu) \gamma$ & 0.00 & 0.00 & 0.00 \\
\hline & $\gamma+$ jets & 156.91 & 0.02 & 0.00 \\
\hline & $e \rightarrow \gamma$ & 0.27 & 0.49 & 2.31 \\
\hline & $j \rightarrow \gamma$ & 18.50 & 2.14 & 1.46 \\
\hline
\end{tabular}

Tabla 9.6: Factores de normalización para los fondos $W \gamma\left(\mu_{W}\right), t \bar{t} \gamma\left(\mu_{T}\right)$ y $\gamma+$ jets $\left(\mu_{Q}\right)$, obtenidos del ajuste combinado en las CR. Las incertezas mostradas son solo estadísticas.

\begin{tabular}{cccc}
\hline & $\mu_{Q}$ & $\mu_{W}$ & $\mu_{T}$ \\
\hline $\mathrm{SR}_{\mathrm{L}}$ & $0.94 \pm 0.44$ & $1.34 \pm 0.89$ & $1.40 \pm 0.77$ \\
$\mathrm{SR}_{\mathrm{H}}$ & $1.22 \pm 0.58$ & $1.24 \pm 0.39$ & $0.54 \pm 0.37$ \\
\hline
\end{tabular}

\subsubsection{Resultados en las regiones de señal}

Las predicciones del fondo luego del ajuste simultáneo en las CR son presentadas en la Tabla 9.9 para las regiones de señal, $\mathrm{SR}_{\mathrm{L}}$ y $\mathrm{SR}_{\mathrm{H}}$, donde se espera señal de nueva física, conjuntamente con el número de eventos de datos observados. De la tabla se desprende que los datos están en buen acuerdo con el fondo esperado y no se observa un exceso estadísticamente significativo de datos por sobre los predichos en ninguna de las SR. El número de eventos esperado a partir de procesos del Modelo Estándar en las regiones $\mathrm{SR}_{\mathrm{L}}$ y $\mathrm{SR}_{\mathrm{H}}$ es de $1.27 \pm 0.43$ y $0.84 \pm 0.38$, respectivamente, mientras que el número de eventos observado en ambas SR es 2.

Las distribuciones de $E_{\mathrm{T}}^{\text {miss }}$ para $\mathrm{SR}_{\mathrm{L}}$ y $\mathrm{SR}_{\mathrm{H}}$ se muestran en la Figura 9.5 para datos y también para el fondo esperado después del ajuste en las CR, y puede verse el buen acuerdo en la zona de 

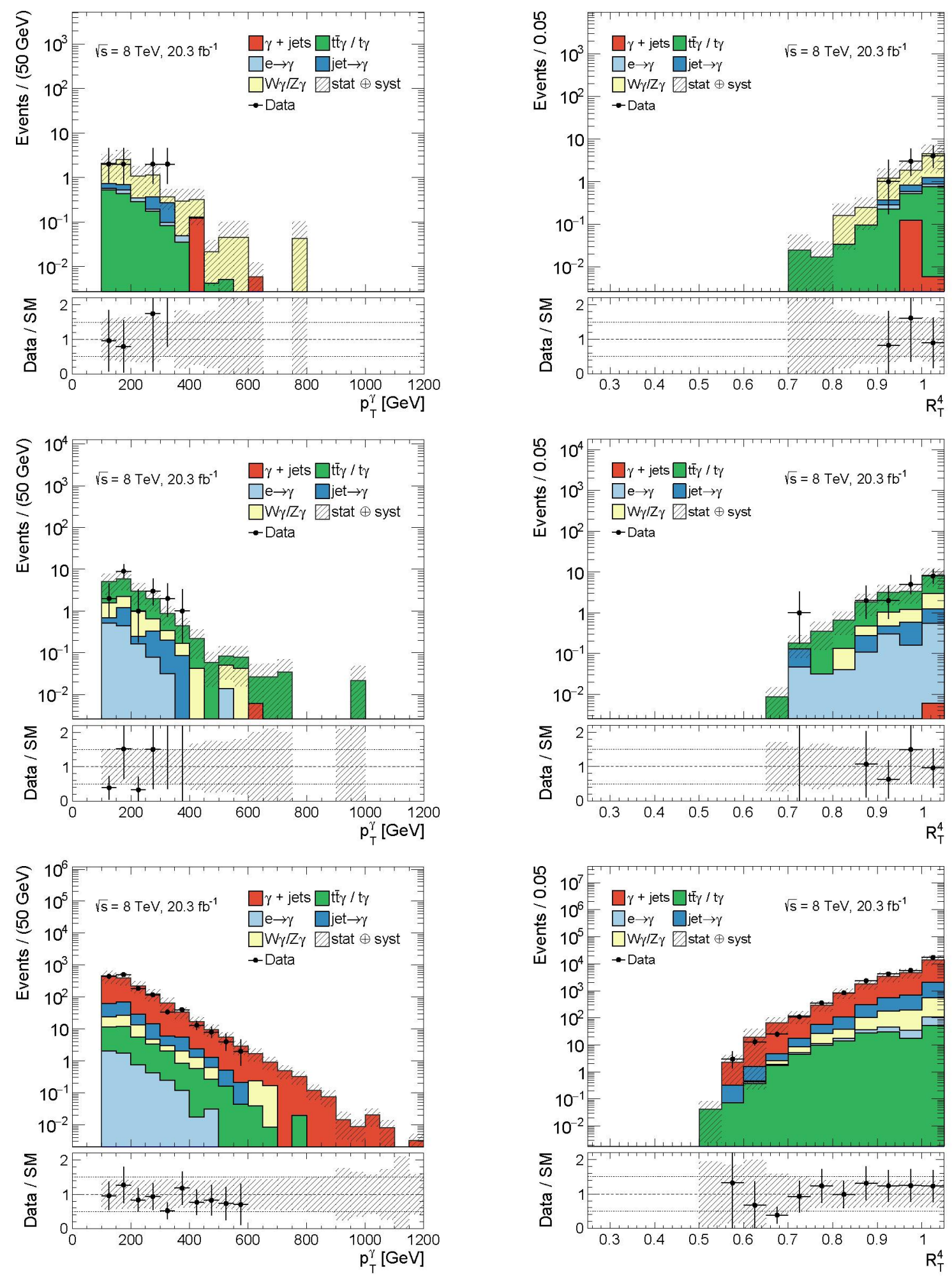

Figura 9.2: Distribuciones observadas de $p_{\mathrm{T}}^{\gamma}$ (izquierda) y $R_{\mathrm{T}}^{4}$ (derecha) en las regiones de control $\mathrm{CRW}_{\mathrm{L}}$ (arriba), $\mathrm{CRT}_{\mathrm{L}}$ (medio) y $\mathrm{CRQ}_{\mathrm{L}}$ (abajo), después del ajuste.

bajo $E_{\mathrm{T}}^{\text {miss }}$. Las flechas indican el corte que define las regiones de señal utilizadas para obtener los resultados finales del presente análisis.

Una comparación entre el número de eventos observado y esperado en cada una de las regiones se presenta en la Figura 9.6 a modo de resumen, donde el panel inferior muestra el pull de cada región, 

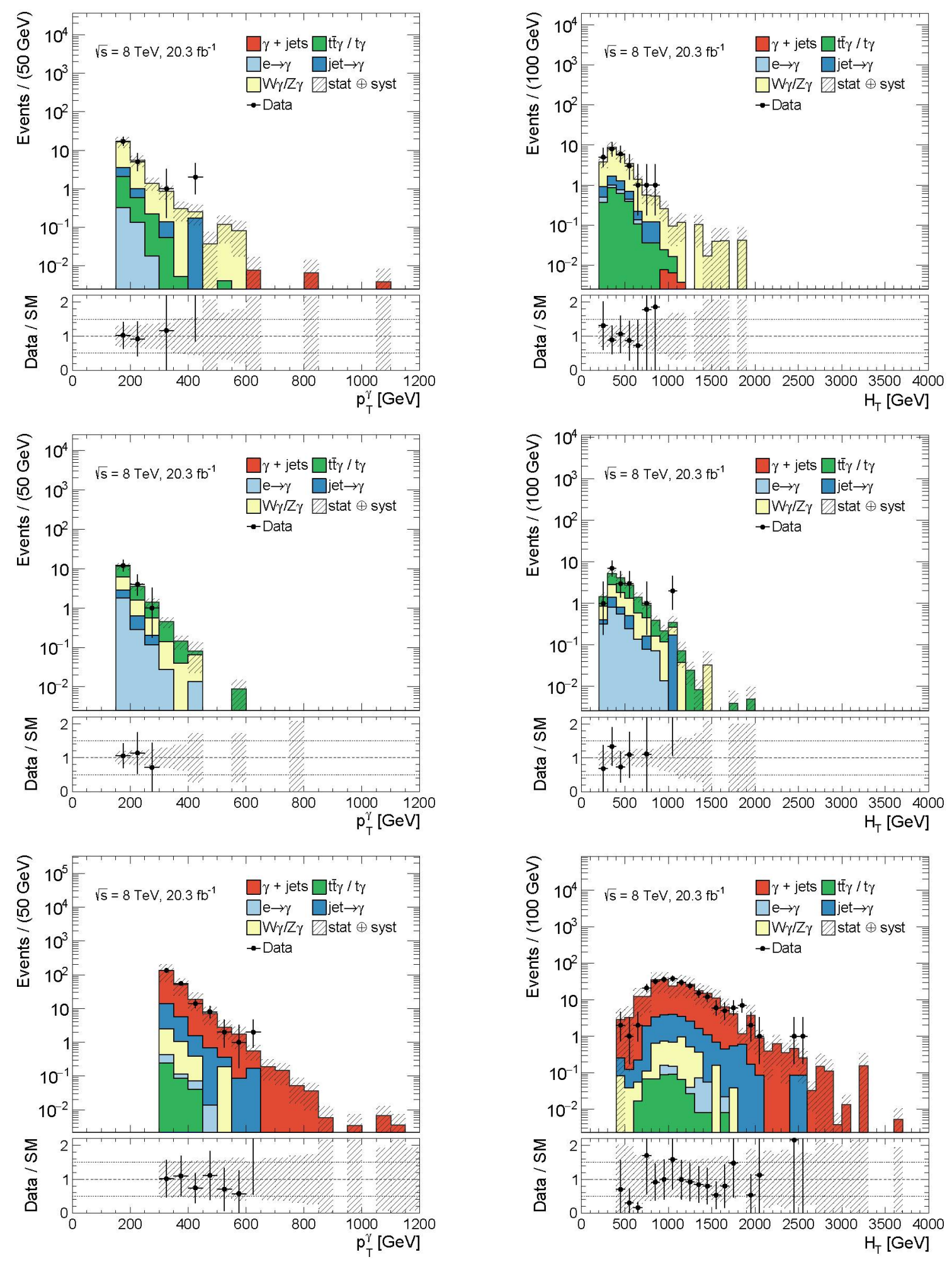

Figura 9.3: Distribuciones observadas de $p_{\mathrm{T}}^{\gamma}$ (izquierda) y $H_{\mathrm{T}}$ (derecha) en las regiones de control $\mathrm{CRW}_{\mathrm{H}}\left(\right.$ arriba), $\mathrm{CRT}_{\mathrm{H}}$ (medio) y $\mathrm{CRQ}_{\mathrm{H}}$ (abajo), después del ajuste.

dando una idea de la significancia estadística de los resultados.

Las incertezas sistemáticas dominantes en el número total predicho de fondo en cada SR, se presentan en la Tabla 9.10. Debido a que las incertezas individuales pueden estar correlacionadas y a las correlaciones negativas entre normalizaciones (representando el impacto de la estadística en las CR 
Tabla 9.7: Resultados del ajuste en las VR correspondientes a $\mathrm{SR}_{\mathrm{L}}$. El número de eventos observado es comparado con el número de eventos esperado de fondo, después de la correspondiente normalización en las CR. Las incertezas incluyen la incerteza estadística y sistemática.

\begin{tabular}{lrrrr}
\hline Regiones de validación & VRQ & VRM75 & VRM100 & VRR \\
\hline Eventos observados & 0 & 54 & 7 & 24 \\
\hline Eventos esperados SM & $0.39 \pm 0.23$ & $51.17 \pm 47.04$ & $13.24 \pm 5.51$ & $24.32 \pm 6.26$ \\
\hline$W \gamma$ & $0.04 \pm 0.04$ & $0.89 \pm 0.79$ & $0.45 \pm 0.40$ & $6.82 \pm 5.74$ \\
$t \bar{t} \gamma$ & $0.30 \pm 0.20$ & $2.55 \pm 1.65$ & $1.54 \pm 1.01$ & $4.74 \pm 2.80$ \\
$t \bar{t} \gamma(\mathrm{had})$ & $0.02 \pm 0.02$ & $1.69 \pm 1.08$ & $0.38 \pm 0.25$ & $0.00 \pm 0.00$ \\
$V(\rightarrow q q) \gamma$ & $0.00 \pm 0.00$ & $2.45 \pm 2.17$ & $0.82 \pm 0.82$ & $0.00 \pm 0.00$ \\
$t \gamma$ & $0.01 \pm 0.01$ & $0.25 \pm 0.10$ & $0.15 \pm 0.06$ & $0.45 \pm 0.08$ \\
$Z(\rightarrow l l) \gamma$ & $0.00 \pm 0.00$ & $0.08_{-0.08}^{+0.08}$ & $0.04_{-0.04}^{+0.04}$ & $0.08_{-0.08}^{+0.08}$ \\
$Z(\rightarrow \nu \nu) \gamma$ & $0.00 \pm 0.00$ & $0.10_{-0.10}^{+0.12}$ & $0.07_{-0.07}^{+0.07}$ & $1.71_{-1.71}^{+1.75}$ \\
$\gamma+$ jets & $0.01_{-0.01}^{+0.07}$ & $35.97_{-35.97}^{+44.72}$ & $7.87 \pm 4.47$ & $2.37 \pm 1.17$ \\
$e \rightarrow \gamma$ & $0.01 \pm 0.00$ & $2.56 \pm 0.72$ & $1.32 \pm 0.43$ & $6.09 \pm 1.14$ \\
$j \rightarrow \gamma$ & $0.00 \pm 0.00$ & $4.63 \pm 2.41$ & $0.60 \pm 0.33$ & $2.06 \pm 0.98$ \\
\hline
\end{tabular}

\begin{tabular}{lrrrr}
\hline Regiones de validación & VRWR & VRWM & VRTR & VRTM \\
\hline Eventos observados & 0 & 5 & 1 & 3 \\
\hline Eventos esperados SM & $0.20 \pm 0.15$ & $3.41 \pm 1.41$ & $0.76 \pm 0.30$ & $3.52 \pm 0.84$ \\
\hline$W \gamma$ & $0.13 \pm 0.10$ & $2.39 \pm 1.47$ & $0.04 \pm 0.03$ & $1.04 \pm 0.65$ \\
$t \bar{t} \gamma$ & $0.04_{-0.04}^{+0.07}$ & $0.41 \pm 0.21$ & $0.46 \pm 0.28$ & $1.86 \pm 0.92$ \\
$t \gamma$ & $0.03 \pm 0.02$ & $0.04 \pm 0.01$ & $0.08 \pm 0.03$ & $0.19 \pm 0.04$ \\
$Z(\rightarrow l l) \gamma$ & $0.00 \pm 0.00$ & $0.06_{-0.06}^{+0.06}$ & $0.02_{-0.02}^{+0.02}$ & $0.00 \pm 0.00$ \\
$e \rightarrow \gamma$ & $0.00 \pm 0.00$ & $0.07 \pm 0.02$ & $0.08 \pm 0.02$ & $0.17 \pm 0.03$ \\
$j \rightarrow \gamma$ & $0.00 \pm 0.00$ & $0.43 \pm 0.21$ & $0.09 \pm 0.04$ & $0.26 \pm 0.12$ \\
\hline
\end{tabular}

en la incerteza total), la incerteza total no es necesariamente la suma en cuadratura de las mismas. La incerteza estadística se ilustra con el valor de la raíz cuadrada del número de eventos esperado con la finalidad de mostrar el impacto relativo de la incerteza estadística con respecto a la incerteza total del fondo. Las incertezas sistemáticas relativas con respecto a las predicciones del fondo son del orden de $54 \%(58 \%)$ para $\mathrm{SR}_{\mathrm{L}}\left(\mathrm{SR}_{\mathrm{H}}\right)$. En $\mathrm{SR}_{\mathrm{L}}$ las incertezas dominantes provienen de la normalización y de las incertezas teóricas del fondo de $t \bar{t} \gamma$, seguidas por la incerteza en la escala de energía de los jets. En $\mathrm{SR}_{\mathrm{H}}$, las incertezas están dominadas por las incertezas teóricas del fondo $Z \gamma$ y $\gamma+$ jets, y la incerteza en la normalización del fondo $W \gamma$.

\subsubsection{Eventos en las regiones de señal}

Solo dos eventos pasan la selección de cada una de las regiones de señal y resulta instructivo una mirada detallada de los mismos. Un esquema del detector y las señales dejadas por los distintos objetos en los diferentes subdetectores puede verse en la Figura 9.7 para los dos eventos observados en la $\mathrm{SR}_{\mathrm{L}}$ y en la Figura 9.8 para los dos eventos en la $\mathrm{SR}_{\mathrm{H}}$.

Las visualizaciones de los eventos es generada con AtLantis [159]. En la parte superior izquierda se puede ver el corte transversal del detector junto con el corte longitudinal en el panel inferior. En estas vistas se presentan los depósitos de energía en los calorímetros en color amarillo. Los conos de distintos colores representan los distintos jets reconstruidos en el evento, y su tamaño es proporcional 
Tabla 9.8: Resultados del ajuste en las VR correspondientes a $\mathrm{SR}_{\mathrm{H}}$. El número de eventos observado es comparado con el número de eventos esperado de fondo, después de la correspondiente normalización en las CR. Las incertezas incluyen la incerteza estadística y sistemática.

\begin{tabular}{|c|c|c|c|c|}
\hline Regiones de validación & & VRQ & & VRH \\
\hline Eventos observados & & 2 & & 4 \\
\hline Eventos esperados SM & & $0.89 \pm 0.32$ & & $3.39 \pm 1.69$ \\
\hline$W \gamma$ & & $0.34 \pm 0.19$ & & $1.18 \pm 0.55$ \\
\hline$t \bar{t} \gamma$ & & $0.07 \pm 0.05$ & & $0.11 \pm 0.08$ \\
\hline$t \bar{t} \gamma(\mathrm{had})$ & & $0.01 \pm 0.01$ & & $0.00 \pm 0.00$ \\
\hline$t \gamma$ & & $0.03 \pm 0.01$ & & $0.01 \pm 0.00$ \\
\hline$Z(\rightarrow l l) \gamma$ & & $0.02_{-0.02}^{+0.02}$ & & $0.00 \pm 0.00$ \\
\hline$Z(\rightarrow \nu \nu) \gamma$ & & $0.08_{-0.08}^{+0.08}$ & & $1.60_{-1.60}^{+1.61}$ \\
\hline$\gamma+$ jets & & $0.19 \pm 0.11$ & & $0.00 \pm 0.00$ \\
\hline$e \rightarrow \gamma$ & & $0.00 \pm 0.00$ & & $0.15 \pm 0.03$ \\
\hline$j \rightarrow \gamma$ & & $0.17 \pm 0.09$ & & $0.34 \pm 0.16$ \\
\hline Regiones de validación & VRWH & VRWM & VRTH & VRTM \\
\hline Eventos observados & 1 & 5 & 2 & 2 \\
\hline Eventos esperados SM & $1.25 \pm 0.38$ & $4.38 \pm 1.14$ & $0.83 \pm 0.26$ & $1.76 \pm 0.38$ \\
\hline$W \gamma$ & $1.08 \pm 0.38$ & $3.75 \pm 1.17$ & $0.28 \pm 0.17$ & $1.08 \pm 0.35$ \\
\hline$t \bar{t} \gamma$ & $0.04 \pm 0.04$ & $0.13 \pm 0.09$ & $0.17 \pm 0.13$ & $0.38 \pm 0.27$ \\
\hline$t \gamma$ & $0.02 \pm 0.01$ & $0.02 \pm 0.01$ & $0.15 \pm 0.04$ & $0.08 \pm 0.02$ \\
\hline$Z(\rightarrow l l) \gamma$ & $0.00 \pm 0.00$ & $0.00 \pm 0.00$ & $0.02_{-0.02}^{+0.02}$ & $0.00 \pm 0.00$ \\
\hline$\gamma+$ jets & $0.02 \pm 0.01$ & $0.00 \pm 0.00$ & $0.00 \pm 0.00$ & $0.00 \pm 0.00$ \\
\hline$e \rightarrow \gamma$ & $0.00 \pm 0.00$ & $0.04 \pm 0.01$ & $0.05 \pm 0.01$ & $0.05 \pm 0.01$ \\
\hline$j \rightarrow \gamma$ & $0.09 \pm 0.04$ & $0.43 \pm 0.20$ & $0.17 \pm 0.09$ & $0.17 \pm 0.08$ \\
\hline
\end{tabular}

Tabla 9.9: Resultados del ajuste en las SR. El número de eventos observado es comparado con el número de eventos esperado de fondo, después de la correspondiente normalización en las CR. Las incertezas incluyen la incerteza estadística y sistemática.

\begin{tabular}{lrr}
\hline Regiones de señal & $\mathrm{SR}_{\mathrm{L}}$ & $\mathrm{SR}_{\mathrm{H}}$ \\
\hline Eventos observados & 2 & 2 \\
\hline Eventos esperados SM & $1.27 \pm 0.43$ & $0.84 \pm 0.38$ \\
\hline$W \gamma$ & $0.13 \pm 0.12$ & $0.54 \pm 0.28$ \\
$t \bar{t} \gamma$ & $0.64 \pm 0.40$ & $0.05 \pm 0.05$ \\
$t \gamma$ & $0.06 \pm 0.02$ & $0.03 \pm 0.01$ \\
$Z(\rightarrow \nu \nu) \gamma$ & $0.03_{-0.03}^{+0.05}$ & $0.21_{-0.21}^{+0.23}$ \\
$\gamma+$ jets & $0.00_{-0.00}^{+0.06}$ & $0.00 \pm 0.00$ \\
$e \rightarrow \gamma$ & $0.38 \pm 0.10$ & $0.00 \pm 0.00$ \\
$j \rightarrow \gamma$ & $0.02_{-0.02}^{+0.08}$ & $0.00_{-0.00}^{+0.08}$ \\
\hline
\end{tabular}

a la energía del mismo. La línea gruesa de color amarillo representa un fotón reconstruido y la línea roja punteada la energía faltante. 

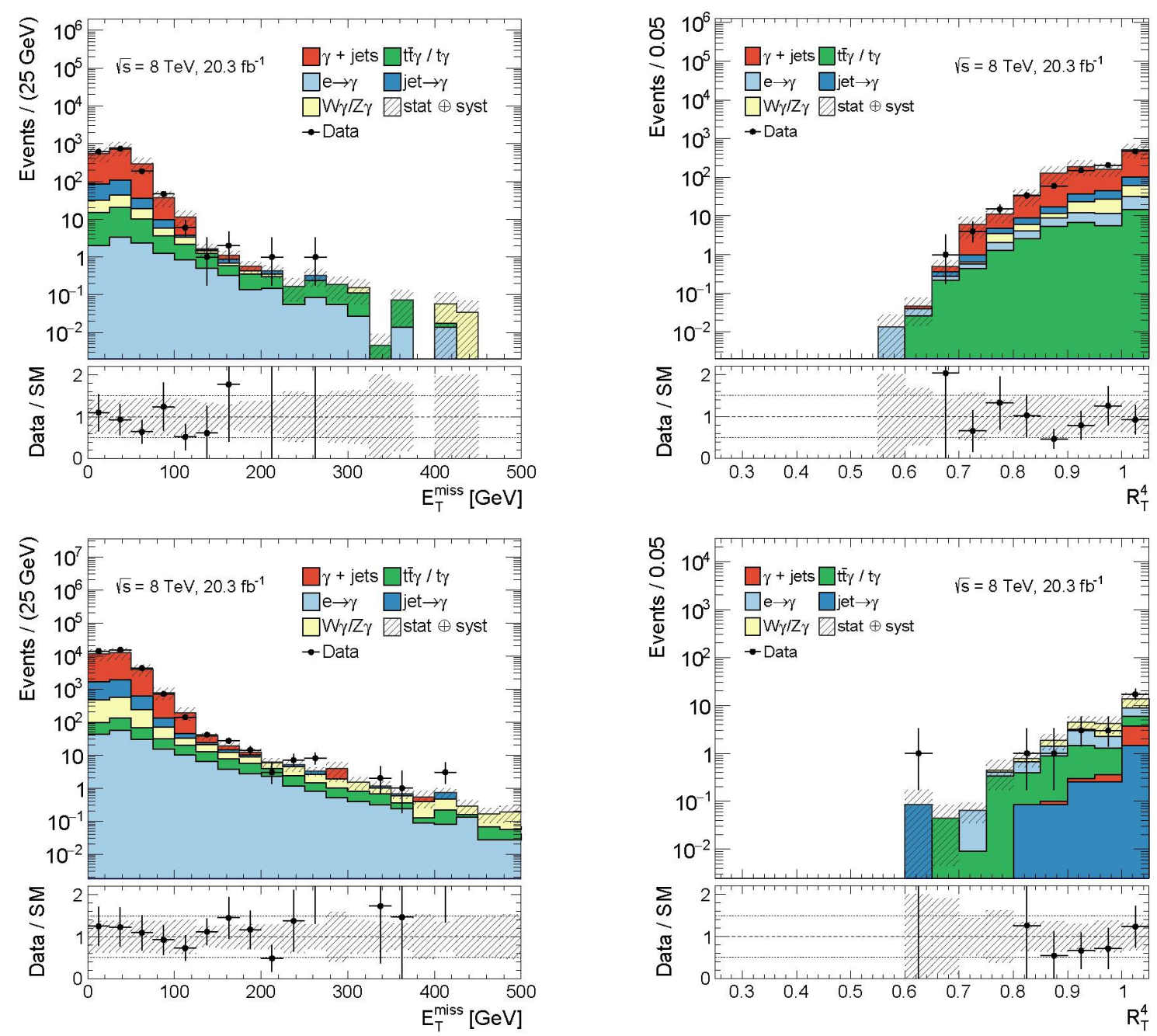

Figura 9.4: Distribuciones observadas de $E_{\mathrm{T}}^{\text {miss }}$ (izquierda) y $R_{\mathrm{T}}^{4}$ (derecha) para algunas de las regiones de validación correspondientes a $\mathrm{SR}_{\mathrm{L}}$ : VRM75 (arriba) y VRR (abajo).
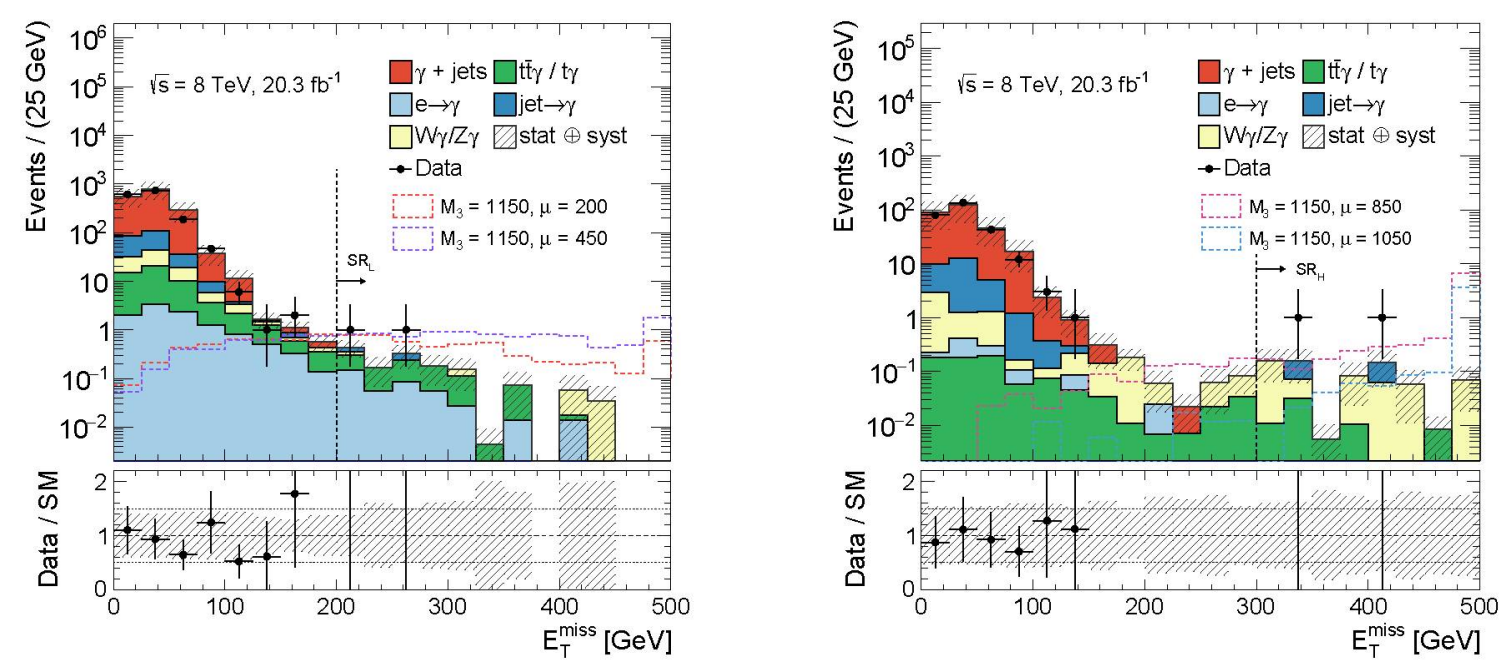

Figura 9.5: Distribución de $E_{\mathrm{T}}^{\text {miss }}$ comparando datos y el fondo esperado para la selección de la región $\mathrm{SR}_{\mathrm{L}}$ (izquierda) y $\mathrm{SR}_{\mathrm{H}}$ (derecha), sin el corte en $E_{\mathrm{T}}^{\text {miss }}$. La distribución esperada para dos muestras de señal es también graficada para su comparación. Las líneas punteadas y las flechas negras indican la región de señal. 

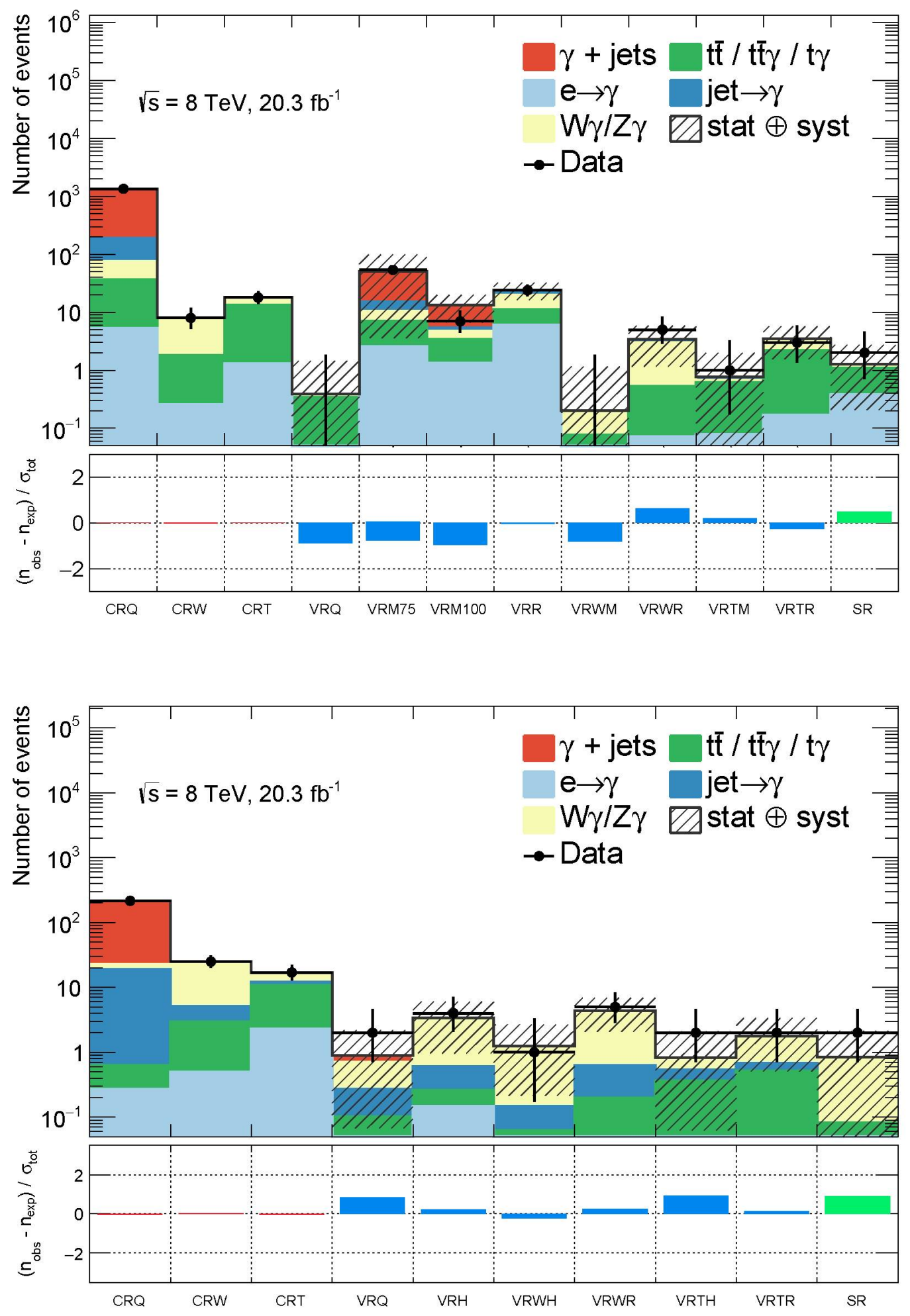

Figura 9.6: Comparación entre el número de eventos observado y esperado en cada una de las regiones correspondientes a $\mathrm{SR}_{\mathrm{L}}$ (arriba) y $\mathrm{SR}_{\mathrm{H}}$ (abajo). El panel inferior muestra el pull definido como $\frac{n_{\text {obs }}-n_{\text {exp }}}{\sigma_{\text {tot }}}$, donde $\sigma_{\text {tot }}$ es la suma de la incerteza estadística y sistemática. 
Tabla 9.10: Resumen de las incertezas sistemáticas dominantes en la estimación del fondo total en $\mathrm{SR}_{\mathrm{L}}$ y $\mathrm{SR}_{\mathrm{H}}$. Notar que las incertezas individuales pueden estar correlacionadas, y la incerteza total no es necesariamente la suma en cuadratura de estas. Los porcentajes muestran el tamaño de la incerteza relativo al fondo esperado total.

\begin{tabular}{lrr}
\hline Incertezas & $\mathrm{SR}_{\mathrm{L}}$ & $\mathrm{SR}_{\mathrm{H}}$ \\
\hline Eventos esperados SM & 1.27 & 0.84 \\
\hline Incerteza estadística total $(\sqrt{N})$ & \pm 1.13 & \pm 0.92 \\
Incerteza sistemática total & $\pm 0.43[34.18 \%]$ & $\pm 0.38[45.27 \%]$ \\
\hline Teoría $t \bar{t} \gamma$ & $\pm 0.36[28.0 \%]$ & $\pm 0.02[1.8 \%]$ \\
$\mu_{T}$ & $\pm 0.35[27.7 \%]$ & $\pm 0.04[4.4 \%]$ \\
Escala de energía de jets & $\pm 0.21[16.5 \%]$ & $\pm 0.03[4.0 \%]$ \\
Teoría $W \gamma$ & $\pm 0.09[7.3 \%]$ & $\pm 0.21[25.1 \%]$ \\
$\mu_{W}$ & $\pm 0.08[6.7 \%]$ & $\pm 0.17[20.5 \%]$ \\
Estimación $j \rightarrow \gamma$ & $\pm 0.08[6.3 \%]$ & $\pm 0.08[9.5 \%]$ \\
Estimación $e \rightarrow \gamma$ & $\pm 0.07[5.7 \%]$ & - \\
Resolución de energía de jets & $\pm 0.06[4.3 \%]$ & $\pm 0.04[4.8 \%]$ \\
Teoría $Z \gamma$ & $\pm 0.03[2.7 \%]$ & $\pm 0.21[25.4 \%]$ \\
Incerteza material $e / \gamma$ & $\pm 0.03[2.2 \%]$ & $\pm 0.04[4.5 \%]$ \\
Resolución de energía de $e / \gamma$ & $\pm 0.03[2.0 \%]$ & $\pm 0.05[5.8 \%]$ \\
Término soft de $E_{\mathrm{T}}^{\text {miss }}$ & $\pm 0.02[1.7 \%]$ & - \\
Corrección por pile-up & $\pm 0.01[0.71 \%]$ & $\pm 0.10[11.9 \%]$ \\
Resolución término soft $E_{\mathrm{T}}^{\text {miss }}$ & $\pm 0.00[0.35 \%]$ & $\pm 0.04[4.5 \%]$ \\
Teoría $t \gamma$ & $\pm 0.00[0.34 \%]$ & $0.00[0.22 \%]$ \\
Eficiencia fotones & $\pm 0.00[0.06 \%]$ & $\pm 0.00[0.40 \%]$ \\
$\mu_{Q}$ & $\pm 0.00[0.02 \%]$ & - \\
Teoría $\gamma j$ & $\pm 0.00[0.02 \%]$ & - \\
\hline
\end{tabular}



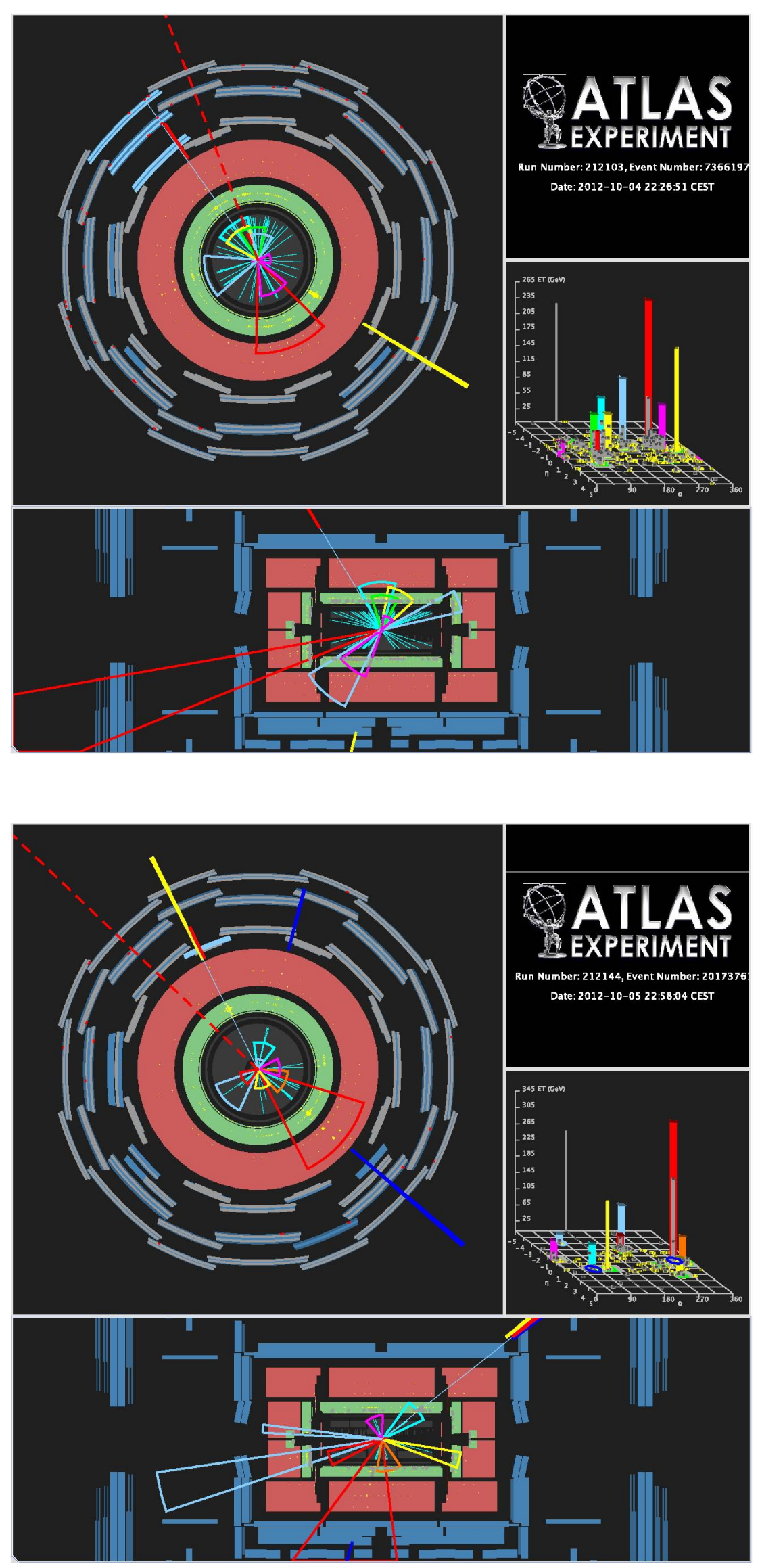

Figura 9.7: Visualización de los dos eventos que sobreviven la selección de $\mathrm{SR}_{\mathrm{L}}$. Ver detalles en el texto. 

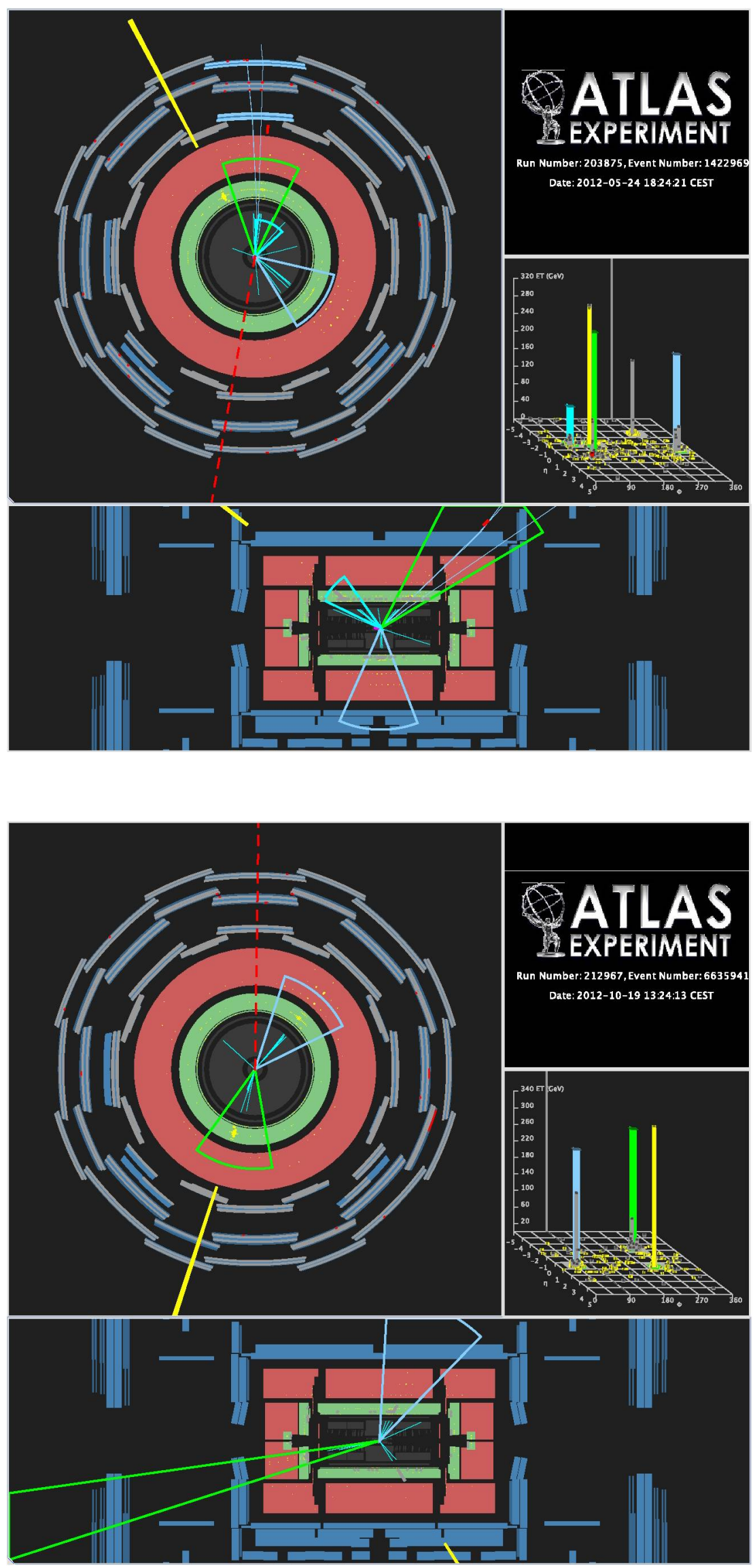

Figura 9.8: Visualización de los dos eventos que sobreviven la selección de $\mathrm{SR}_{\mathrm{H}}$. Ver detalles en el texto. 


\subsection{Límites a procesos de nueva física}

Una vez obtenidos los resultados finales en las regiones de señal en términos del número de eventos observado en relación al fondo esperado como se discutió en las secciones anteriores, resulta útil proveer el límite superior en el número de eventos de nueva física impuesto por dichos resultados. Este límite, obtenido de manera independiente de cualquier modelo, es provisto para cada región de señal, haciendo posible su aplicación a cualquier modelo de nueva física. Para esto debe calcularse el número de eventos de señal que se espera en la región en cuestión y verificar que dicho número no exceda el límite superior del presente análisis. Por supuesto que los valores de eficiencia y aceptancia deben ser tenidos en cuenta. Para que puedan realizarse este tipo de interpretaciones en base a otros modelos, todos los valores y datos relevantes de este análisis son publicados en HepData para su utilización [160].

Con el objetivo de calcular los límites independientes del modelo se realiza un ajuste combinado teniendo en cuenta las regiones de control y cada una de las regiones de señal. Se supone que la contaminación de señal en las CR es nula, pero no se realiza ninguna otra suposición acerca del modelo de nueva física. El número de eventos de señal en la SR se agrega como el parámetro de interés y se deja libre en el ajuste. Se realiza un muestreo de este parámetro, y el límite superior en el número de eventos de nueva física se encuentra para el valor en el cual el $\mathrm{CL}_{s}$ cae debajo del $5 \%$. El límite superior en el número de eventos puede transformarse en el límite superior de la sección eficaz visible* utilizando la luminosidad considerada en el análisis $\left(20.3 \mathrm{fb}^{-1}\right)$.

Los resultados se detallan en la Tabla 9.11 para las dos regiones de señal. Debido a que el número esperado de eventos de fondo es bajo y no es posible utilizar la aproximación asintótica que fuera descripta en la sección 4.7, se utilizaron $\sim 3000$ pseudo-experimentos generados con el método Monte Carlo. El límite en el número de eventos de nueva física resulta en 5.5 y 5.6 para $\mathrm{SR}_{\mathrm{L}}$ y $\mathrm{SR}_{\mathrm{H}}$ respectivamente, lo cual se traduce en un límite en la sección eficaz visible de 0.27 y $0.28 \mathrm{fb}$.

Tabla 9.11: Límite independiente del modelo de señal a $95 \%$ de CL en la sección eficaz visible observada $\left(\langle\epsilon \sigma\rangle_{\text {obs }}\right)$, y el límite en el número de eventos de nueva física observado $S_{\text {obs }}$ para las dos SR. La última línea $\left(p_{0}\right)$ indica el valor- $p$ de la hipótesis de solo-fondo.

\begin{tabular}{lrr}
\hline Regiones de señal & $\mathrm{SR}_{\mathrm{L}}$ & $\mathrm{SR}_{\mathrm{H}}$ \\
\hline Eventos observados & 2 & 2 \\
Eventos esperados SM & $1.27 \pm 0.43$ & $0.84 \pm 0.38$ \\
\hline$\langle\epsilon \sigma\rangle_{\text {obs }}[\mathrm{fb}]$ & 0.27 & 0.28 \\
$S_{\mathrm{obs}}$ & 5.5 & 5.6 \\
$p_{0}$ & 0.16 & 0.19 \\
\hline
\end{tabular}

\footnotetext{
${ }^{*}$ La sección eficaz visible tiene en cuenta la aceptancia y eficiencia de selección del análisis, es decir, $\sigma_{\mathrm{vis}}=\epsilon \sigma$
} 


\subsection{Límites de exclusión en el modelo de SUSY considerado}

Debido a que no se observó un exceso significativo por encima del fondo esperado del Modelo Estándar en las regiones de señal, se obtuvieron los límites de exclusión en el modelo de SUSY considerado. Los límites superiores son determinados con el método $\mathrm{CL}_{s}$ descripto en la sección 4.6. Para ello se utiliza la función likelihood, ecuación (9.1), incluyendo cada región de señal y las muestras de señal del modelo de SUSY que motivó este estudio. Estos límites son calculados para la sección eficaz nominal del modelo y también con una sección eficaz de $\pm 1 \sigma$ en la incerteza teórica, siguiendo la recomendación de ATLAS y el acuerdo de los experimentos del LHC para presentar los resultados.

La Figura 9.9 muestra los límites de exclusión esperados y observados para las dos SR de forma separada. Los límites fueron obtenidos utilizando $\sim 20000$ pseudo-experimentos. La línea punteada azul muestra el límite esperado a $95 \%$ CL, con las bandas amarillas indicando la desviación a $1 \sigma$ teniendo en cuenta las incertezas teóricas y experimentales. Los límites observados están indicados por las líneas en rojo oscuro, donde la línea sólida representa el límite nominal y las líneas punteadas representan el límite para las variaciones de la sección eficaz de la señal debido a la incerteza teórica en la misma.

Por diseño, la $\mathrm{SR}_{\mathrm{L}}$ es importante en la zona de neutralinos livianos, mientras que la $\mathrm{SR}_{\mathrm{H}}$ cubre la región más comprimida del espacio de fases. En la Figura 9.10, se muestra el límite combinando ambas regiones de señal, eligiendo en cada punto la que provee el mejor límite.
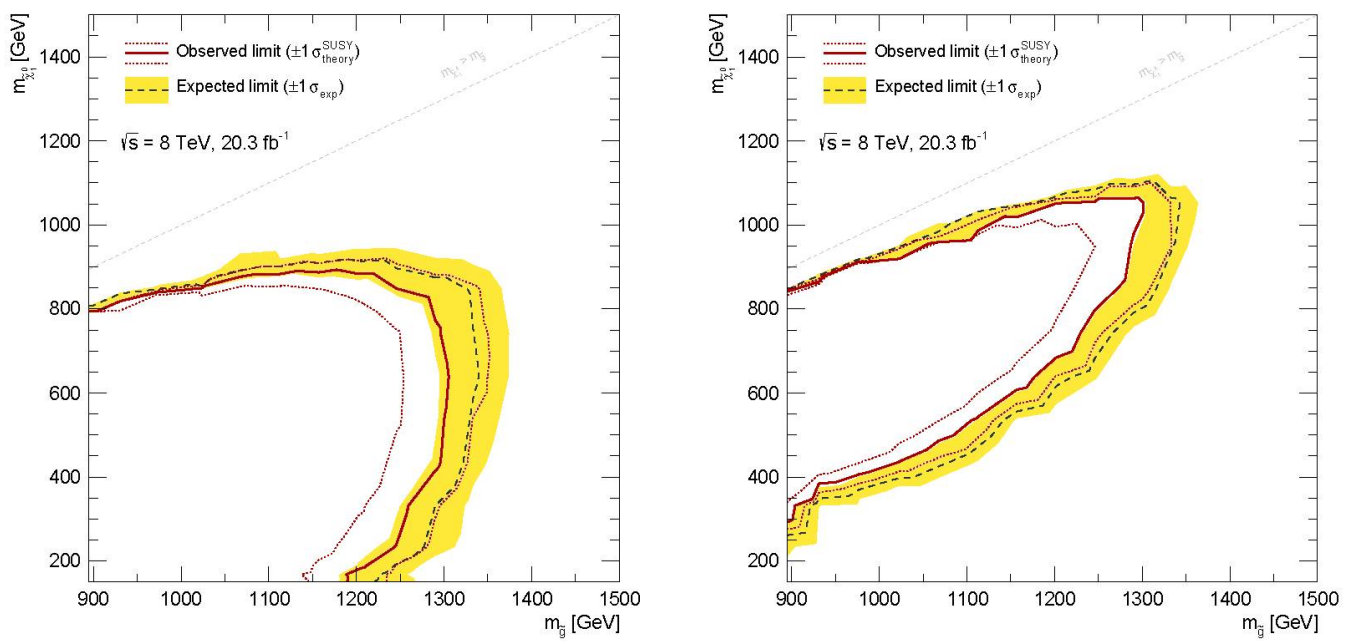

Figura 9.9: Límites de exclusión a $95 \%$ CL para $\mathrm{SR}_{\mathrm{L}}$ (izquierda) y $\mathrm{SR}_{\mathrm{H}}$ (derecha).

Como se desprende de los gráficos de los límites de exclusión, considerando el contorno de $-1 \sigma$ en la sección eficaz de SUSY, la producción de gluino es excluida a 95\% CL para una masa de hasta 1250 $\mathrm{GeV}$, para $m_{\widetilde{\chi}_{1}^{0}}<840 \mathrm{GeV}$. Este límite disminuye para neutralinos de baja masa debido a la caída en la aceptancia en esa región, lo cual también ocurre cuando la masa de gluinos y neutralinos es similar.

A modo de completitud, en la Figura 9.11 se presenta un resumen de los límites obtenidos en las masas de las partículas supersimétricas buscadas en una selección de los análisis más sensibles realizados con datos de ATLAS. La figura incluye el resultado obtenido por el análisis presentado en esta tesis, al igual que los análisis complementarios de búsqueda de modelos GGM. Puede observarse que los límites obtenidos, incluida la contribución de esta tesis, alcanzan y superan la región del TeV para la masa de gluino. 


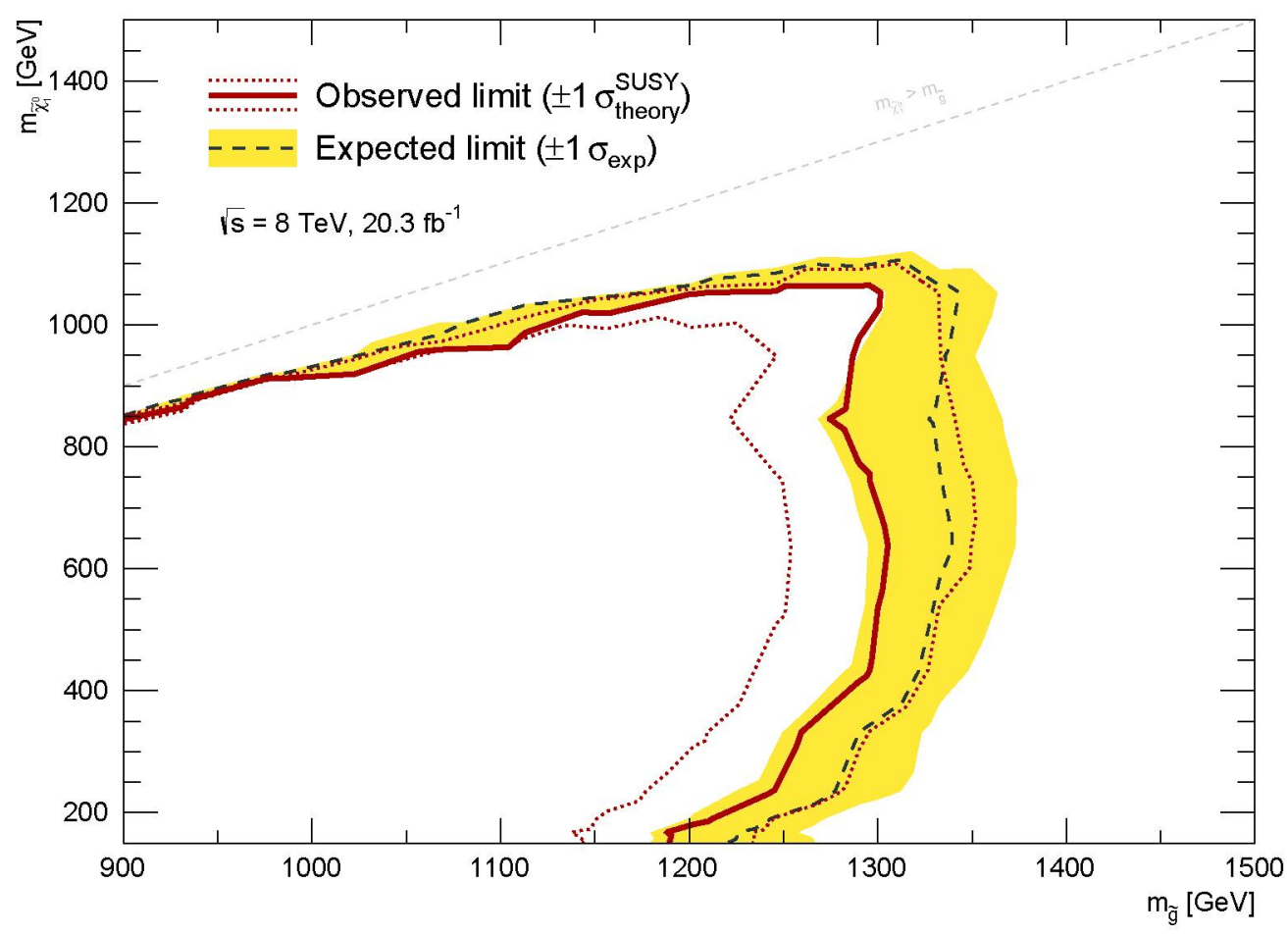

Figura 9.10: Límites de exclusión para $\mathrm{SR}_{\mathrm{L}}$ y $\mathrm{SR}_{\mathrm{H}}$ combinados. Los límites son obtenidos usando la región de señal con mejor sensibilidad en cada punto. La línea punteada azul muestra el límite esperado a $95 \% \mathrm{CL}$, con las bandas amarillas indicando la desviación a $1 \sigma$ que tiene en cuenta las incertezas teóricas y experimentales. Los límites observados están indicados por las líneas en rojo oscuro, donde la línea sólida representa el límite nominal y las líneas punteadas representan el límite para las variaciones de la sección eficaz de la señal debido a la incerteza teórica en la misma.

\subsection{Búsqueda de señal de SUSY con datos a $\sqrt{s}=13 \mathrm{TeV}$}

Luego de la publicación de los resultados obtenidos con los datos de $\sqrt{s}=8 \mathrm{TeV}$, presentados en las secciones anteriores, se comenzó el análisis de los nuevos datos a partir de las colisiones del llamado Run 2 del LHC a una energía de centro de masa sin precedente, de $\sqrt{s}=13 \mathrm{TeV}$. Desde Junio a Diciembre del año 2015 el LHC funcionó a esta energía de centro de masa y el detector ATLAS recolectó datos correspondientes a una luminosidad total integrada de $3.2 \mathrm{fb}^{-1}$.

Además del cambio en la energía de las colisiones, se llevaron a cabo varias mejoras en el LHC y sus detectores. También se realizaron mejoras en el trigger, y en el software de reconstrucción y análisis. Durante los dos años que hubo entre las tomas de datos, el LHC fue mejorado ampliamente para poder aumentar la energía de los haces de protones a $6.5 \mathrm{TeV}$, y disminuir el espaciado entre bunches a $25 \mathrm{~ns}$. Al detector ATLAS también se le realizaron mejoras significativas durante este período. Se renovaron el tubo del haz, el sistema de imanes y los sistemas criogénicos. También se reemplazaron o reacondicionaron los módulos del detector interno y los calorímetros dañados, y para mejorar la reconstrucción de trazas y de vértices a luminosidades mayores, se agregó una cuarta capa al detector de píxeles a una distancia de $3.3 \mathrm{~cm}$ del tubo del haz.

El aumento en la energía de las colisiones conlleva a un incremento en la sección eficaz de producción, mejorando el potencial de descubrimiento para física más allá del SM. En particular para SUSY, la sección eficaz esperada de producción de gluinos incrementa en un factor de 30 a 130 en el rango de masas 1.5-2 TeV. En la Figura 9.12 puede verse la comparación entre la sección eficaz para las distintas energías de colisión. 


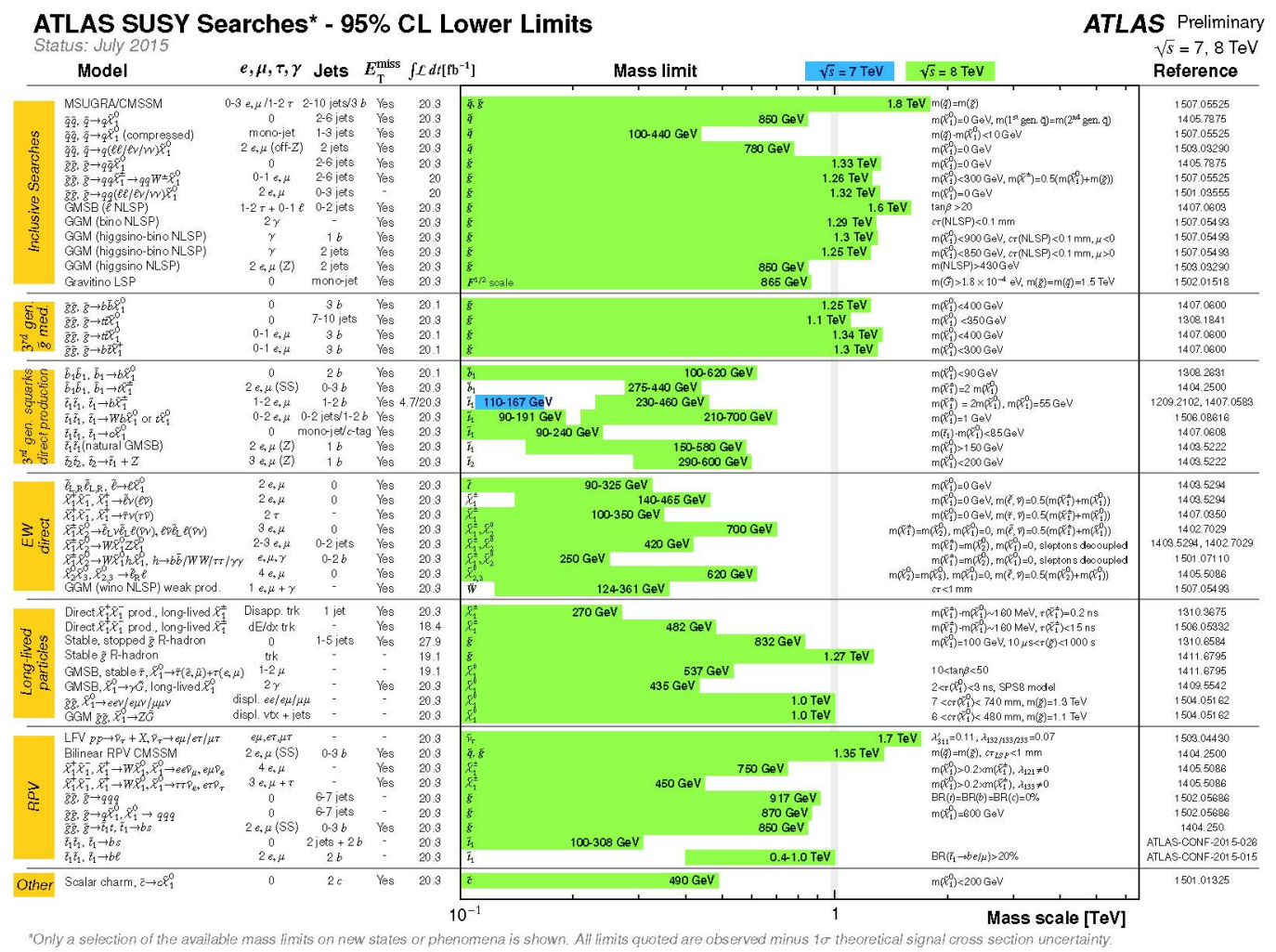

Figura 9.11: Resumen de los límites de exclusión en la masa de las partículas supersimétricas por distintos análisis realizados en ATLAS. Solo una selección representativa de los resultados disponibles es presentada [161], incluyendo los obtenidos en esta tesis.

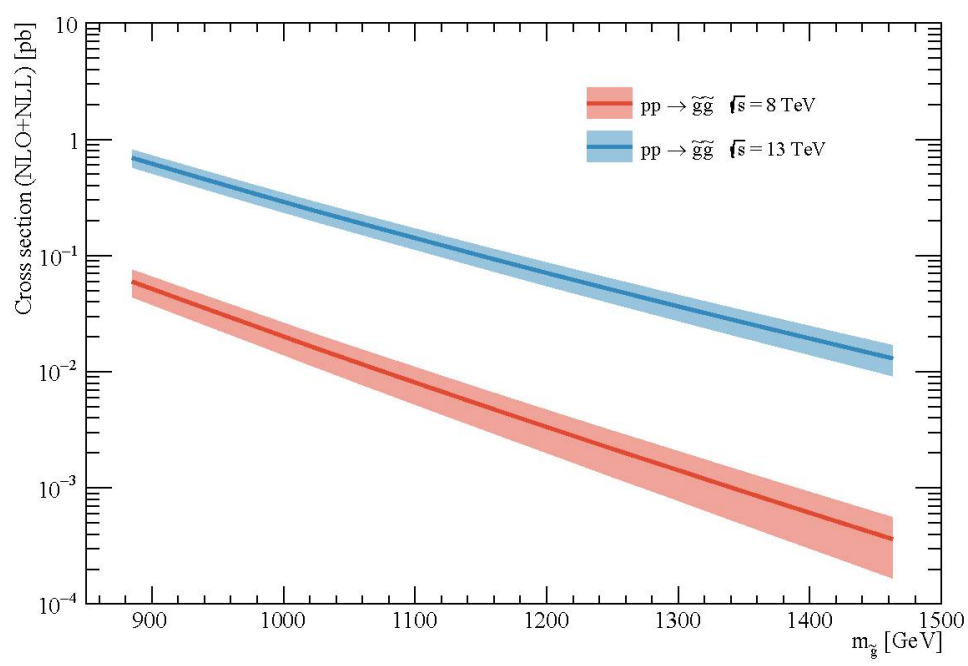

Figura 9.12: Sección eficaz de producción de pares de gluinos como función de la masa del gluino para energías de centro de masa de $8 \mathrm{TeV}$ y $13 \mathrm{TeV}$.

Para el nuevo análisis se diseñó un conjunto de puntos de señal (ver Figura 9.13), utilizando el mismo modelo considerado previamente y explicado en la sección 6.2 , aunque extendiendo el espacio de parámetros. Los mismos fueron generados utilizando Herwig ++ . En este caso, la masa de los squarks se fijo en $5 \mathrm{TeV}$, mientras que todos los demás parámetros se mantuvieron iguales a la grid anterior utilizada en esta tesis. 

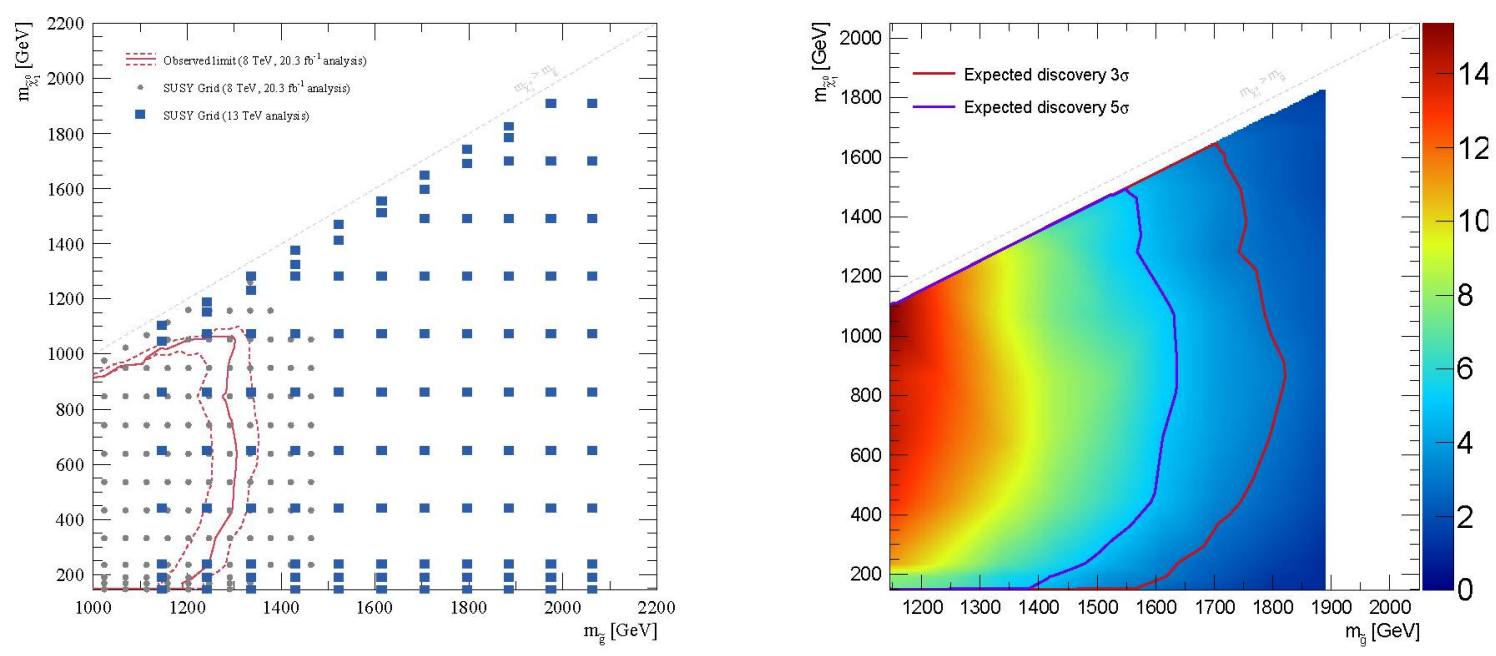

Figura 9.13: Izquierda: Conjunto de puntos (grid) de señal simulados para el análisis de Run 2. Los cuadrados azules representan los puntos de señal generados con distintos valores de $m_{\widetilde{g}}$ y $m_{\tilde{\chi}_{1}^{0}}$. Derecha: Significancia de descubrimiento esperada para el análisis de datos de $13 \mathrm{TeV}$, para una luminosidad total integrada de $4 \mathrm{fb}^{-1}$. Los contornos se corresponden con $3 \sigma$ y $5 \sigma$.

Con las muestras generadas de señal y de los procesos del SM, y a partir de la selección de las dos regiones de señal $\mathrm{SR}_{\mathrm{L}}$ y $\mathrm{SR}_{\mathrm{H}}$ del análisis de Run 1 , se optimizaron nuevamente los cortes, considerando una luminosidad total esperada de $4 \mathrm{fb}^{-1}$. El resultado de los cortes de selección de eventos es el que se detalla en la Tabla 9.12. El cambio más relevante respecto a la selección previa es la introducción del corte en $M_{\text {eff }}=H_{\mathrm{T}}+E_{\mathrm{T}}^{\text {miss }}$. En la Tabla 9.12 puede verse además el fondo esperado para estas dos regiones de señal, considerando una luminosidad total integrada de $4 \mathrm{fb}^{-1}$.

Tabla 9.12: Izquierda: Selección final para las regiones de señal $\mathrm{SR}_{\mathrm{L}}$ y $\mathrm{SR}_{\mathrm{H}}$ optimizadas para el análisis de los datos de $\sqrt{s}=13 \mathrm{TeV}$. Derecha: Fondo esperado para región de señal, para una luminosidad total integrada de $4 \mathrm{fb}^{-1}$.

\begin{tabular}{lrr}
\hline & $\mathrm{SR}_{\mathrm{L}}$ & $\mathrm{SR}_{\mathrm{H}}$ \\
\hline$N_{\text {fotones }}$ & $=1$ & $=1$ \\
$p_{\mathrm{T}}^{\gamma}>$ & $145 \mathrm{GeV}$ & $400 \mathrm{GeV}$ \\
$N_{\text {leptones }}$ & 0 & 0 \\
$N_{\text {jets }}>$ & 4 & 2 \\
$\Delta \phi\left(\right.$ jet, $\left.E_{\mathrm{T}}^{\text {miss }}\right)>$ & 0.4 & 0.4 \\
$\Delta \phi\left(\gamma, E_{\mathrm{T}}^{\mathrm{miss}}\right)>$ & 0.4 & 0.4 \\
$E_{\mathrm{T}}^{\text {miss }}>$ & $200 \mathrm{GeV}$ & $400 \mathrm{GeV}$ \\
$M_{\text {eff }}>$ & $2000 \mathrm{GeV}$ & $2000 \mathrm{GeV}$ \\
$R_{T}^{4}<$ & 0.90 & - \\
\hline
\end{tabular}

\begin{tabular}{lrr}
\hline & $\mathrm{SR}_{\mathrm{L}}$ & $\mathrm{SR}_{\mathrm{H}}$ \\
\hline$\gamma+$ jet & $0.01 \pm 0.00$ & $0.04 \pm 0.01$ \\
multijet & $0.00 \pm 0.00$ & $0.01 \pm 0.00$ \\
$W \gamma$ & $0.17 \pm 0.08$ & $0.15 \pm 0.07$ \\
$Z(l l) \gamma$ & $0.00 \pm 0.00$ & $0.01 \pm 0.01$ \\
$Z(\nu \nu) \gamma$ & $0.01 \pm 0.01$ & $0.09 \pm 0.05$ \\
$t \bar{t} \gamma$ & $0.08 \pm 0.08$ & $0.15 \pm 0.10$ \\
$W+$ jets & $0.00 \pm 0.00$ & $0.03 \pm 0.02$ \\
$Z+$ jets & $0.00 \pm 0.00$ & $0.07 \pm 0.05$ \\
\hline Fondo total & $0.27 \pm 0.17$ & $0.55 \pm 0.30$ \\
\hline
\end{tabular}

En la Figura 9.13 (derecha) se encuentra la significancia esperada de descubrimiento, donde las líneas representan los contornos de $3 \sigma$ y $5 \sigma$. El análisis de datos se está llevando a cabo al presente y se espera que los resultados sean presentados en las conferencias de mediados de 2016 y publicados hacia fin de año. 


\section{CONCLusiones}

Supersimetría (SUSY) es una de las extensiones del Modelo Estándar de las partículas fundamentales y sus interacciones más atractivas que se encuentra bajo estudio en el Gran Colisionador de Hadrones (LHC) del CERN. El descubrimiento de un nuevo escalar compatible con el bosón de Higgs del SM por los experimentos ATLAS [9] y CMS del LHC permitió establecer límites a los modelos de SUSY y el escenario de búsqueda ha cambiado a partir de este resultado. A fin de realizar la interpretación de los resultados de búsquedas de Supersimetría en el LHC se consideraron diferentes modelos para el mecanismo del rompimiento de SUSY, el cual es desconocido, de modo de reducir el número de parámetros libres imponiendo ciertos límites fenomenológicos.

El análisis realizado en esta tesis constituye la primera búsqueda de nueva física en eventos con un fotón aislado de alto $p_{\mathrm{T}}$, jets y energía faltante, utilizando los datos recolectados durante colisiones $p p$ a $\sqrt{s}=8 \mathrm{TeV}$ por el detector ATLAS en el LHC. Este estado final se corresponde con una de las señales predichas en modelos de rompimiento de SUSY con mediación por campos de gauge (GGM, General Gauge Mediation), donde el neutralino NLSP es una mezcla higgsino-bino con aproximadamente igual tasa de decaimiento a fotones y al bosón $Z$. Los datos utilizados corresponden a una luminosidad total integrada de $20.3 \mathrm{fb}^{-1}$, y los resultados obtenidos se incluyeron en la primera publicación de ATLAS en este tema [162]. La experiencia ganada en las contribuciones realizadas en el estadio inicial del doctorado, aportando al análisis en los estados finales $\gamma \gamma+E_{\mathrm{T}}^{\mathrm{miss}}[26,27]$ y $\gamma+e / \mu+E_{\mathrm{T}}^{\mathrm{miss}}[28]$, resultó valiosa para el desarrollo del análisis que constituyó la investigación central de esta tesis.

El mayor desafío para la realización de cualquier descubrimiento de nueva física es la definición de una región en el espacio de observables donde se espera que domine la señal por sobre el fondo del Modelo Estándar y la estimación de dicho fondo en esa región. En esta tesis se definieron dos regiones de señal: La primera, $\mathrm{SR}_{\mathrm{L}}$, motivada por el decaimiento de gluinos en neutralinos de baja/media masa, cuyos eventos están caracterizados por una gran multiplicidad de jets, mientras que la energía del fotón y la energía faltante quedan determinadas por la masa del neutralino. La otra, $\mathrm{SR}_{\mathrm{H}}$, destinada a cubrir el espacio de parámetros en el que la masa del neutralino y el gluino es similar, con eventos que se caracterizan por un fotón de alto $p_{\mathrm{T}}$ y una gran cantidad de $E_{\mathrm{T}}^{\mathrm{miss}}$.

Para determinar los fondos contaminantes fue necesario el uso de diversas simulaciones Monte Carlo y el desarrollo de métodos específicos para estimar fondos a partir de los propios datos de colisiones.

El tratamiento estadístico de los datos merece también una mención aparte, dado que constituye un aspecto fundamental en el análisis de datos en experimentos de altas energías. Muchos de los métodos estadísticos utilizados fueron especialmente desarrollados para las búsquedas de nueva física en ATLAS, como la presentada en esta tesis.

Otro de los ingredientes importantes del análisis de datos lo constituyen las incertezas sistemáticas que afectan la señal y la determinación del fondo. Se incluyeron los efectos provenientes de las limitaciones de la física dentro de las simulaciones del detector, aquellos originados en los métodos 
de reconstrucción e identificación de las partículas, como también las incertezas provenientes de las predicciones teóricas.

Todos estos ingredientes necesarios para el análisis fueron incorporados al marco estadístico utilizado a fin de obtener los resultados finales. En cada región de señal se observaron 2 eventos con una predicción para el fondo de $1.27 \pm 0.43$ y $0.84 \pm 0.38$ para la región $\mathrm{SR}_{\mathrm{L}}$ y $\mathrm{SR}_{\mathrm{H}}$, respectivamente.

Dado que no se observó un exceso estadísticamente significativo por sobre las predicciones del Modelo Estándar, se establecieron límites a las posibles contribuciones de nueva física. Estos límites a 95\% CL en el número de eventos provenientes de nueva física son de 5.5 y 5.6 para las dos regiones de señal definidas en el análisis, los cuales se corresponden a límites en la sección eficaz visible de 0.27 y $0.28 \mathrm{fb}$.

Adicionalmente, se realizó una interpretación de los resultados en el contexto de un modelo de GGM SUSY con el estado final que motivó esta búsqueda, excluyendo a $95 \%$ CL la producción de gluinos con una masa de hasta $1.25 \mathrm{TeV}$, dependiendo de la masa del $\widetilde{\chi}_{1}^{0}$.

En paralelo con la edición de esta tesis, se está llevando a cabo el análisis de búsqueda de nueva física en el estado final de un fotón de alto $p_{\mathrm{T}}$, jets y energía faltante con el conjunto total de datos recolectados por ATLAS durante el año 2015, correspondiente a una luminosidad integrada de $\sim 3 \mathrm{fb}^{-1}$ a $\sqrt{s}=13 \mathrm{TeV}$. La operación del LHC a mayores energías incrementa la sección eficaz de producción de partículas supersimétricas, de modo se espera que estas nuevas investigaciones extiendan la región de sensibilidad y permitan tener, quizás, evidencias de presencia de señales en la región de gluinos de alta masa. 


\section{AGRADECIMIENTOS}

La verdad es que esta tesis no hubiera sido posible sin el apoyo de muchos. A todos los que recuerdo y a todos los que me olvido... ;Gracias!

A Tere, por darme la oportunidad de trabajar en lo que me gusta, y en el lugar soñado. Por la física, los viajes, las cervezas, los acentos, las anécdotas repetidas y las frases acertadas. Por la confianza y fundamentalmente por estar para todo, siempre. Al Tincho, porque este análisis no hubiera sido posible sin él. A todo el grupo de trabajo. A los de siempre, a los que se fueron y a los que llegaron. Porque cada uno aporta algo para crear un increíble ambiente de trabajo, y logran que uno tenga ganas de ir a la oficina todas las mañanas. Por los primeros tutoriales de ROOT, las charlas, los scripts, por saber siempre cual es la release correcta y por las correcciones a la parte de teoría de esta tesis. Por las reuniones, las discusiones y por las preguntas que casi siempre suelen ser más útiles que las respuestas. A todo el grupo de SUSY de ATLAS porque semana a semana guiaron este análisis. Por las preguntas, los deadline, las infinitas presentaciones y los detalles que solo ellos eran capaces de ver. A la UNLP, la Facultad de Ciencias Exactas, el Departamento de Física y el IFLP. Al CONICET por financiar este doctorado. A los compañeros y amigos que conocí a lo largo de todo este tiempo. A mi familia, a mis papás, mi hermana y mi abuela, porque siempre hicieron todo lo posible para darme lo mejor, y gracias a ellos hoy llegué hasta acá. A jo, por todos los momentos que compartimos, por estar siempre y por aguantarme a mí y a esta tesis. 


\section{Bibliografía}

[1] L. R. Evans y P. Bryant, LHC Machine, J. Instrum. 3 (2008) S08001. 164 p. https://cds.cern.ch/record/1129806. This report is an abridged version of the LHC Design Report (CERN-2004-003).

[2] ATLAS Collaboration, The ATLAS Experiment at the CERN Large Hadron Collider, Journal of Instrumentation 3 no. 08, (2008) S08003.

http://stacks.iop.org/1748-0221/3/i=08/a=S08003.

[3] ATLAS Collaboration, Performance of the ATLAS Trigger System in 2010, Eur. Phys. J. C72 (2012) 1849, arXiv:1110.1530.

[4] S. Weinberg, A Model of Leptons, Phys. Rev. Lett. 19 (1967) 1264-1266. http://link.aps.org/doi/10.1103/PhysRevLett.19.1264.

[5] J. Goldstone, A. Salam, y S. Weinberg, Broken Symmetries, Phys. Rev. 127 (1962) 965-970. http://link.aps.org/doi/10.1103/PhysRev.127.965.

[6] S. L. Glashow, Partial-symmetries of weak interactions, Nucl. Phys. 22 no. 4, (1961) $579-588$. http://www. sciencedirect.com/science/article/pii/0029558261904692.

[7] F. Englert y R. Brout, Broken Symmetry and the Mass of Gauge Vector Mesons, Phys. Rev. Lett. 13 (1964) 321-323. http://link.aps.org/doi/10.1103/PhysRevLett.13. 321.

[8] P. W. Higgs, Broken Symmetries and the Masses of Gauge Bosons, Phys. Rev. Lett. 13 (1964) 508-509. http://link.aps.org/doi/10.1103/PhysRevLett.13.508.

[9] ATLAS Collaboration, Observation of a new particle in the search for the Standard Model Higgs boson with the ATLAS detector at the LHC, Phys.Lett. B716 (2012) 1-29, arXiv:1207.7214.

[10] H. Miyazawa, Baryon Number Changing Currents, Prog. Theor. Phys. 36 (6) (1966) 1266-1276.

[11] P. Ramond, Dual Theory for Free Fermions, Phys. Rev. D3 (1971) 2415-2418.

[12] Y. A. Gol'fand y E. P. Likhtman, Extension of the Algebra of Poincare Group Generators and Violation of $p$ Invariance, JETP Lett. 13 (1971) 323-326.

[13] A. Neveu y J. H. Schwarz, Factorizable dual model of pions, Nucl. Phys. B31 (1971) 86-112.

[14] A. Neveu y J. H. Schwarz, Quark Model of Dual Pions, Phys. Rev. D4 (1971) 1109-1111.

[15] J. Gervais y B. Sakita, Field theory interpretation of supergauges in dual models, Nucl. Phys. B34 (1971) 632-639.

[16] D. V. Volkov y V. P. Akulov, Is the Neutrino a Goldstone Particle?, Phys. Lett. B 46 (1973) $109-110$. 
[17] J. Wess y B. Zumino, A Lagrangian Model Invariant Under Supergauge Transformations, Phys. Lett. B 49 (1974) 52.

[18] J. Wess y B. Zumino, Supergauge Transformations in Four-Dimensions, Nucl. Phys. B70 (1974) $39-50$.

[19] M. Dine y W. Fischler, A Phenomenological Model of Particle Physics Based on Supersymmetry, Phys. Lett. B 110 (1982) 227.

[20] L. Alvarez-Gaume, M. Claudson, y M. B. Wise, Low-Energy Supersymmetry, Nucl. Phys. B207 (1982) 96 .

[21] C. R. Nappi y B. A. Ovrut, Supersymmetric Extension of the $S U(3) \times S U(2) \times U(1)$ Model, Phys. Lett. B 113 (1982) 175.

[22] M. Dine y A. E. Nelson, Dynamical supersymmetry breaking at low-energies, Phys. Rev. D48 (1993) 1277-1287, arXiv:hep-ph/9303230.

[23] M. Dine, A. E. Nelson, y Y. Shirman, Low-energy dynamical supersymmetry breaking simplified, Phys. Rev. D51 (1995) 1362-1370, arXiv:hep-ph/9408384.

[24] M. Dine, A. E. Nelson, Y. Nir, y Y. Shirman, New tools for low-energy dynamical supersymmetry breaking, Phys. Rev. D53 (1996) 2658-2669, arXiv:hep-ph/9507378.

[25] P. Meade, N. Seiberg, y D. Shih, General Gauge Mediation, Prog. Theor. Phys. Suppl. 177 (2009) 143-158, arXiv:0801.3278.

[26] ATLAS Collaboration, Search for diphoton events with large missing transverse momentum in of 7 TeV proton-proton collision data with the ATLAS detector, Phys. Lett. B 710 no. 4-5, (2012) $519-537$. http://www. sciencedirect.com/science/article/pii/s0370269312001955.

[27] ATLAS Collaboration, Search for Diphoton Events with Large Missing Transverse Momentum in 8 TeV pp Collision Data with the ATLAS Detector, Tech. Rep. ATLAS-CONF-2014-001, CERN, Geneva, Jan, 2014.

[28] ATLAS Collaboration, Search for supersymmetry in events with at least one photon, one lepton, and large missing transverse momentum in proton-proton collision at a center-of-mass energy of 7 TeV with the ATLAS detector, Tech. Rep. ATLAS-CONF-2012-144, CERN, Geneva, Nov, 2012.

[29] ATLAS Collaboration, Search for supersymmetry in events with photons, bottom quarks, and missing transverse momentum in proton-proton collisions at a centre-of-mass energy of $7 \mathrm{TeV}$ with the ATLAS detector, Phys.Lett. B 719 (2013) 261-279, arXiv:1211.1167.

[30] ATLAS Collaboration, Search for supersymmetry in final states with jets, missing transverse momentum and $a Z$ boson at $\sqrt{s}=8 \mathrm{TeV}$ with the ATLAS detector, Tech. Rep. ATLAS-CONF-2012-152, CERN, Geneva, Nov, 2012.

[31] CMS Collaboration, Search for supersymmetry in events with photons and missing energy, Tech. Rep. CMS-PAS-SUS-12-018, CERN, Geneva, 2012.

[32] CMS Collaboration, Search for supersymmetry in events with one photon, jets and missing transverse energy at $\sqrt{s}=8 \mathrm{TeV}$, Tech. Rep. CMS-PAS-SUS-14-004, CERN, Geneva, 2014.

[33] Particle Data Group Collaboration, Review of Particle Physics, Chin. Phys. C38 (2014) 090001. 
[34] CMS Collaboration, Observation of a new boson at a mass of $125 \mathrm{GeV}$ with the CMS experiment at the LHC, Phys.Lett. B716 (2012) 30-61, arXiv:1207.7235.

[35] ATLAS, CMS Collaboration, Combined Measurement of the Higgs Boson Mass in pp Collisions at $\sqrt{s}=7$ and $8 \mathrm{TeV}$ with the ATLAS and CMS Experiments, Phys. Rev. Lett. 114 (2015) 191803 , arXiv: 1503.07589 .

[36] R. K. Ellis, W. J. Stirling, y B. R. Webber, QCD and collider physics, Camb. Monogr. Part. Phys. Nucl. Phys. Cosmol. 8 (1996) 1-435.

[37] R. P. Feynman, Very High-Energy Collisions of Hadrons, Phys. Rev. Lett. 23 (1969) 1415-1417. http://link.aps.org/doi/10.1103/PhysRevLett.23.1415.

[38] J. D. Bjorken y E. A. Paschos, Inelastic Electron-Proton and $\gamma$-Proton Scattering and the Structure of the Nucleon, Phys. Rev. 185 (1969) 1975-1982. http://link.aps.org/doi/10.1103/PhysRev.185.1975.

[39] R. Ellis, H. Georgi, M. Machacek, H. Politzer, y G. G. Ross, Factorization and the parton model in QCD, Physics Letters B 78 no. 2-3, (1978) $281-284$.

http://www.sciencedirect.com/science/article/pii/0370269378900230.

[40] V. N. Gribov y L. N. Lipatov, Deep inelastic ep scattering in perturbation theory, Sov. J. Nucl. Phys. 15 (1972) 438-450. [Yad. Fiz.15,781(1972)].

[41] L. N. Lipatov, The parton model and perturbation theory, Sov. J. Nucl. Phys. 20 (1975) 94-102. [Yad. Fiz.20,181(1974)].

[42] G. Altarelli y G. Parisi, Asymptotic freedom in parton language, Nuclear Physics B 126 no. 2, (1977) $298-318$. http://www.sciencedirect.com/science/article/pii/0550321377903844.

[43] ATLAS Collaboration, "Standard Model Summary Plots." https://atlas . web. cern. ch/Atlas/GROUPS/PHYSICS/CombinedSummaryPlots/SM/, 2015.

[44] R. Ciesielski, Charged and neutral current cross sections from HERA, Journal of Physics: Conference Series 110 no. 4, (2008) 042007.

http://stacks . iop.org/1742-6596/110/i=4/a=042007.

[45] SNO Collaboration, Independent Measurement of the Total Active ${ }^{8} \mathrm{~B}$ Solar Neutrino Flux Using an Array of ${ }^{3} \mathrm{He}$ Proportional Counters at the Sudbury Neutrino Observatory, Phys. Rev. Lett. 101 (2008) 111301. http://link.aps.org/doi/10.1103/PhysRevLett.101.111301.

[46] The Super-Kamiokande Collaboration, Solar neutrino measurements in Super-Kamiokande-II, Phys. Rev. D 78 (2008) 032002. http://link.aps.org/doi/10.1103/PhysRevD.78.032002.

[47] F. Zwicky, On the Masses of Nebulae and of Clusters of Nebulae, Astrophysical Journal86 .

[48] M. S. T. E. W. Kolb, The early universe,.

[49] S. P. Martin, A Supersymmetry primer, arXiv:hep-ph/9709356 [hep-ph]. [Adv. Ser. Direct. High Energy Phys.18,1(1998)].

[50] H. E. Haber, Introductory low-energy supersymmetry, in Theoretical Advanced Study Institute (TASI 92): From Black Holes and Strings to Particles Boulder, Colorado, June 3-28, 1992. 1993. arXiv: hep-ph/9306207. 
[51] S. Dimopoulos y D. W. Sutter, The Supersymmetric flavor problem, Nucl. Phys. B452 (1995) 496-512, arXiv:hep-ph/9504415.

[52] G. F. Giudice y R. Rattazzi, Theories with gauge mediated supersymmetry breaking, Phys. Rept. 322 (1999) 419-499, arXiv: hep-ph/9801271.

[53] P. Meade, M. Reece, y D. Shih, Prompt Decays of General Neutralino NLSPs at the Tevatron, JHEP 05 (2010) 105, arXiv:0911.4130.

[54] H. Pagels y J. R. Primack, Supersymmetry, Cosmology, and New Physics at Teraelectronvolt Energies, Phys. Rev. Lett. 48 (1982) 223-226. http://link.aps.org/doi/10.1103/PhysRevLett.48.223.

[55] T. Moroi, H. Murayama, y M. Yamaguchi, Cosmological constraints on the light stable gravitino, Phys. Lett. B303 (1993) 289-294.

[56] J. T. Ruderman y D. Shih, General Neutralino NLSPs at the Early LHC, JHEP 08 (2012) 159, arXiv: 1103.6083

[57] W. Beenakker, R. Hopker, y M. Spira, PROSPINO: A Program for the production of supersymmetric particles in next-to-leading order QCD, arXiv:hep-ph/9611232 [hep-ph].

[58] LEP design report. CERN, Geneva, 1983. https://cds.cern.ch/record/98881. By the LEP Injector Study Group.

[59] ATLAS Collaboration, N. Wermes y G. Hallewel, ATLAS pixel detector: Technical Design Report. Technical Design Report ATLAS. CERN, Geneva, 1998. https://cds.cern. ch/record/381263.

[60] ATLAS liquid-argon calorimeter: Technical Design Report. Technical Design Report ATLAS. CERN, Geneva, 1996.

[61] ATLAS tile calorimeter: Technical Design Report. Technical Design Report ATLAS. CERN, Geneva, 1996.

[62] ATLAS muon spectrometer: Technical Design Report. Technical Design Report ATLAS. CERN, Geneva, 1997. distribution.

[63] ATLAS Collaboration, ATLAS Computing: technical design report. Technical Design Report ATLAS. CERN, Geneva, 2005. https://cds.cern.ch/record/837738.

[64] ATLAS Collaboration, B. Lenzi, The Physics Analysis Tools project for the ATLAS experiment, Tech. Rep. ATL-SOFT-PROC-2009-006, CERN, Geneva, Oct, 2009. http://cds. cern. ch/record/1214931.

[65] P. Calafiura, W. Lavrijsen, C. Leggett, M. Marino, y D. Quarrie, The Athena Control Framework in Production, New Developments and Lessons Learned, https://cds. cern. ch/record/865624.

[66] G. Barrand, I. Belyaev, P. Binko, M. Cattaneo, R. Chytracek, G. Corti, M. Frank, G. Gracia, J. Harvey, E. Herwijnen, P. Maley, P. Mato, S. Probst, y F. Ranjard, GAUDI - A software architecture and framework for building HEP data processing applications, Computer Physics Communications 140 no. 1-2, (2001) $45-55$.

http://www.sciencedirect.com/science/article/pii/S0010465501002545. CHEP2000. 
[67] R. Brun y F. Rademakers, ROOT. An object oriented data analysis framework, Nucl. Inst. and Meth. in Phys. Research Section A 389 no. 1-2, (1997) 81-86.

http://www. sciencedirect.com/science/article/pii/S016890029700048X.

[68] https:

//twiki.cern.ch/twiki/bin/viewauth/AtlasProtected/GoodRunListsForAnalysis.

[69] ATLAS Collaboration, Preliminary Luminosity Determination in pp Collisions at $\sqrt{s}=8 \mathrm{TeV}$ using the ATLAS detector in 2012, tech. rep., Nov, 2012.

https://cds.cern. ch/record/1494059.

[70] https://twiki.cern.ch/twiki/bin/view/AtlasPublic/LuminosityPublicResults.

[71] M. Delmastro, S. Gleyzer, C. Hengler, M. Jimenez, T. Koffas, M. Kuna, K. Liu, Y. Liu, G. Marchiori, E. Petit, M. Pitt, E. Soldatov, y K. Tackmann, Photon identification efficiency measurements with the ATLAS detector using LHC Run 1 data, Tech. Rep.

ATL-COM-PHYS-2014-949, CERN, Geneva, Aug, 2014.

https://cds.cern.ch/record/1747242.

[72] D. Banfi, L. Carminati, J. del Peso, N. Kerschen, L. Mandelli, D. Nebot, y E. Paganis, The measurement of the energy of electrons and photons in the ATLAS electromagnetic calorimeters, Tech. Rep. ATL-PHYS-INT-2010-038, CERN, Geneva, Apr, 2010.

[73] Electron efficiency measurements with the ATLAS detector using the 2012 LHC proton-proton collision data, Tech. Rep. ATLAS-CONF-2014-032, CERN, Geneva, Jun, 2014. https://cds. cern. ch/record/1706245.

[74] ATLAS Collaboration, Measurements of the photon identification efficiency with the ATLAS detector using $4.9 \mathrm{fb}^{1}$ of pp collision data collected in 2011, Tech. Rep. ATLAS-CONF-2012-123, CERN, Geneva, Aug, 2012.

[75] Expected photon performance in the ATLAS experiment, Tech. Rep. ATL-PHYS-PUB-2011-007, CERN, Geneva, Apr, 2011.

[76] https://twiki.cern.ch/twiki/bin/viewauth/AtlasProtected/STACODocumentation.

[77] M. Cacciari, G. P. Salam, y G. Soyez, The Anti-k(t) jet clustering algorithm, JHEP 0804 (2008) 063, arXiv:0802.1189.

[78] W. Lampl, S. Laplace, D. Lelas, P. Loch, H. Ma, S. Menke, S. Rajagopalan, D. Rousseau, S. Snyder, y G. Unal, Calorimeter Clustering Algorithms: Description and Performance, Tech. Rep. ATL-LARG-PUB-2008-002. ATL-COM-LARG-2008-003, CERN, Geneva, Apr, 2008.

[79] ATLAS Collaboration, Jet energy measurement with the ATLAS detector in proton-proton collisions at $\sqrt{s}=7$ TeV, Eur. Phys. J. C73 no. 3, (2013) 2304, arXiv:1112.6426.

[80] E. Abat et al., Combined performance studies for electrons at the 2004 ATLAS combined test-beam, JINST 5 (2010) P11006.

[81] M. Aharrouche et al., Measurement of the response of the ATLAS liquid argon barrel calorimeter to electrons at the 2004 combined test-beam, Nucl. Instrum. Meth. A614 (2010) 400-432.

[82] P. Adragna et al., Testbeam studies of production modules of the ATLAS tile calorimeter, Nucl. Instrum. Meth. A606 (2009) 362-394. 
[83] J. Pinfold et al., Performance of the ATLAS liquid argon endcap calorimeter in the pseudorapidity region $2.5<|\eta|<4.0$ in beam tests, Nucl. Instrum. Meth. A593 (2008) 324-342.

[84] C. Cojocaru, J. Pinfold, et al., Hadronic calibration of the ATLAS liquid argon end-cap calorimeter in the pseudorapidity region $1.6<|\eta|<1.8$ in beam tests, Nuclear Instruments and Methods in Physics Research A 531 (2004) 481-514, physics/0407009.

[85] ATLAS Collaboration, Measurement of inclusive jet and dijet cross sections in proton-proton collisions at $7 \mathrm{TeV}$ centre-of-mass energy with the ATLAS detector, Eur. Phys. J. C71 (2011) 1512, arXiv: 1009.5908.

[86] ATLAS Collaboration, Data-Quality Requirements and Event Cleaning for Jets and Missing Transverse Energy Reconstruction with the ATLAS Detector in Proton-Proton Collisions at a Center-of-Mass Energy of $\sqrt{s}=7$ TeV, Tech. Rep. ATLAS-CONF-2010-038, CERN, Geneva, Jul, 2010. http://cds.cern.ch/record/1277678.

[87] Calibration of the performance of b-tagging for $c$ and light-flavour jets in the 2012 ATLAS data, Tech. Rep. ATLAS-CONF-2014-046, CERN, Geneva, Jul, 2014.

http://cds.cern. ch/record/1741020.

[88] ATLAS Collaboration, A. Collaboration, Measurement of the b-tag efficiency in a sample of jets containing muons with $5 \mathrm{fb}^{-1}$ of data from the ATLAS detector, Tech. Rep.

ATLAS-CONF-2012-043, CERN, Geneva, Mar, 2012.

[89] T. J. Khoo, M. H. Klein, H. Okawa, D. Schaefer, y S. Williams, Reconstruction and Performance of Missing Transverse Momentum in the ATLAS Detector using Proton-Proton Collisions at $\sqrt{s}=8 \mathrm{TeV}$, Tech. Rep. ATL-COM-PHYS-2015-341, CERN, Geneva, Apr, 2015. https://cds.cern.ch/record/2012749.

[90] G. Cowan, Statistical Data Analysis. Oxford Science Publications, 1998.

[91] F. James, Statistical Methods in Experimental Physics. World Scientific, 2008.

[92] G. Cowan, K. Cranmer, E. Gross, y O. Vitells, Asymptotic formulae for likelihood-based tests of new physics, The European Physical Journal C 71 no. 2, (2011). http://dx.doi.org/10.1140/epjc/s10052-011-1554-0.

[93] A. L. Read, Presentation of search results: the $C L_{s}$ technique, Journal of Physics G: Nuclear and Particle Physics 28 no. 10, (2002) 2693. http://stacks . iop.org/0954-3899/28/i=10/a=313.

[94] G. Cowan, "Discovery sensitivity for a counting experiment with background uncertainty." http://www.pp.rhul.ac.uk/ cowan/stat/notes/medsigNote.pdf, 2012.

[95] K. Cranmer, Practical Statistics for the LHC, pp. 267-308. 2014. arXiv:1503.07622.

[96] ROOT Collaboration, K. Cranmer, G. Lewis, L. Moneta, A. Shibata, y W. Verkerke, HistFactory: A tool for creating statistical models for use with RooFit and RooStats, Tech. Rep. CERN-OPEN-2012-016, New York U., New York, Jan, 2012. https://cds.cern.ch/record/1456844.

[97] M. Baak, G. Besjes, D. Côté, A. Koutsman, J. Lorenz, y D. Short, HistFitter software framework for statistical data analysis, The European Physical Journal C 75 no. 4, (2015). http://dx.doi.org/10.1140/epjc/s10052-015-3327-7. 
[98] J. Neyman y E. S. Pearson, On the Problem of the Most Efficient Tests of Statistical Hypotheses, Philosophical Transactions of the Royal Society of London A: Mathematical, Physical and Engineering Sciences 231 no. 694-706, (1933) 289-337.

[99] S. S. Wilks, The Large-Sample Distribution of the Likelihood Ratio for Testing Composite Hypotheses, Ann. Math. Statist. 9 no. 1, (1938) 60-62. http://dx.doi.org/10.1214/aoms/1177732360.

[100] A. Wald, Tests of Statistical Hypotheses Concerning Several Parameters When the Number of Observations is Large, Transactions of the American Mathematical Society 54 no. 3, (1943) pp. 426-482. http://www.jstor.org/stable/1990256.

[101] T. Sjostrand, Monte Carlo Generators, in High-energy physics. Proceedings, European School, Aronsborg, Sweden, June 18-July 1, 2006, pp. 51-74. 2006. arXiv:hep-ph/0611247. http://weblib.cern.ch/abstract?CERN-LCGAPP-2006-06.

[102] M. A. Dobbs et al., Les Houches guidebook to Monte Carlo generators for hadron collider physics, in Physics at TeV colliders. Proceedings, Workshop, Les Houches, France, May 26-June 3, 2003, pp. 411-459. 2004. arXiv: hep-ph/0403045.

http://lss.fnal.gov/cgi-bin/find_paper.pl?conf-04-183.

[103] H. Baer, Computational Tools for Supersymmetry Calculations, Adv. Ser. Direct. High Energy Phys. 21 (2010) 446-468, arXiv:0912.3270.

[104] P. Z. Skands, B. Allanach, H. Baer, C. Balazs, G. Belanger, et al., SUSY Les Houches accord: Interfacing SUSY spectrum calculators, decay packages, and event generators, JHEP 0407 (2004) 036, arXiv:hep-ph/0311123.

[105] A. Djouadi, M. Muhlleitner, y M. Spira, Decays of supersymmetric particles: The Program SUSY-HIT (SUspect-SdecaY-Hdecay-InTerface), Acta Phys.Polon. B38 (2007) 635-644, arXiv:hep-ph/0609292.

[106] A. Djouadi, J.-L. Kneur, y G. Moultaka, SuSpect: A Fortran code for the Supersymmetric and Higgs particle spectrum in the $\{M S S M\}$, Computer Physics Communications 176 no. 6, (2007) $426-455$.

[107] M. Muhlleitner, SDECAY: A Fortran code for SUSY particle decays in the MSSM, Acta Phys.Polon. B35 (2004) 2753-2766, arXiv:hep-ph/0409200.

[108] A. Djouadi, J. Kalinowski, y M. Spira, HDECAY: A Program for Higgs boson decays in the standard model and its supersymmetric extension, Comput.Phys.Commun. 108 (1998) 56-74, arXiv: hep-ph/9704448.

[109] D. Bourilkov, R. C. Group, y M. R. Whalley, LHAPDF: PDF use from the Tevatron to the LHC, in TeV4LHC Workshop - 4th meeting Batavia, Illinois, October 20-22, 2005. 2006. arXiv: hep-ph/0605240.

[110] A. Buckley, J. Ferrando, S. Lloyd, K. Nordström, B. Page, M. Rüfenacht, M. Schönherr, y G. Watt, LHAPDF6: parton density access in the LHC precision era, Eur. Phys. J. C75 (2015) 132, arXiv: 1412.7420 [hep-ph].

[111] e. a. Pavel M. Nadolsky, Implications of CTEQ global analysis for collider observation, Phys. Rev. D78 (2008) 013004, arXiv: 0802.0007. 
[112] ATLAS Collaboration, The ATLAS Simulation Infrastructure, The European Physical Journal C 70 no. 3, (2010) 823-874. http://dx.doi.org/10.1140/epjc/s10052-010-1429-9.

[113] S. A. et al., Geant4: A simulation toolkit, Nuclear Instruments and Methods in Physics Research Section A: Accelerators, Spectrometers, Detectors and Associated Equipment 506 no. 3, (2003) $250-303$.

[114] E. Richter-Was, D. Froidevaux, y L. Poggioli, ATLFAST 2.0 a fast simulation package for ATLAS, Tech. Rep. ATL-PHYS-98-131, CERN, Geneva, Nov, 1998.

[115] N. Craig, D. Green, y A. Katz, (De)Constructing a Natural and Flavorful Supersymmetric Standard Model, JHEP 1107 (2011) 045, arXiv: 1103.3708.

[116] R. Auzzi, A. Giveon, y S. B. Gudnason, Flavor of quiver-like realizations of effective supersymmetry, JHEP 1202 (2012) 069, arXiv:1112.6261.

[117] C. Csaki, L. Randall, y J. Terning, Light Stops from Seiberg Duality, Phys.Rev. D86 (2012) 075009, arXiv: 1201.1293.

[118] G. Larsen, Y. Nomura, y H. L. Roberts, Supersymmetry with Light Stops, JHEP 1206 (2012) 032 , arXiv: 1202.6339

[119] N. Craig, S. Dimopoulos, y T. Gherghetta, Split families unified, JHEP 1204 (2012) 116, arXiv: 1203.0572.

[120] M. Bahr, S. Gieseke, M. Gigg, D. Grellscheid, K. Hamilton, et al., Herwig ++ Physics and Manual, Eur.Phys.J. C58 (2008) 639-707, arXiv:0803.0883.

[121] M. Kramer et al., Supersymmetry production cross sections in pp collisions at $\sqrt{s}=7$ TeV, arXiv: 1206.2892.

[122] W. Beenakker, R. Hopker, M. Spira, y P. M. Zerwas, Squark and gluino production at hadron colliders, Nucl. Phys. B492 (1997) 51-103, arXiv: hep-ph/9610490.

[123] A. Kulesza y L. Motyka, Threshold resummation for squark-antisquark and gluino-pair production at the LHC, Phys. Rev. Lett. 102 (2009) 111802, arXiv:0807.2405.

[124] A. Kulesza y L. Motyka, Soft gluon resummation for the production of gluino-gluino and squark-antisquark pairs at the LHC, Phys. Rev. D80 (2009) 095004, arXiv:0905.4749.

[125] W. Beenakker, S. Brensing, M. Kramer, A. Kulesza, E. Laenen, y I. Niessen, Soft-gluon resummation for squark and gluino hadroproduction, JHEP 12 (2009) 041, arXiv:0909.4418.

[126] W. Beenakker, S. Brensing, M. n. Kramer, A. Kulesza, E. Laenen, L. Motyka, y I. Niessen, Squark and Gluino Hadroproduction, Int. J. Mod. Phys. A26 (2011) 2637-2664, arXiv: 1105.1110.

[127] M. Botje, J. Butterworth, A. Cooper-Sarkar, A. de Roeck, J. Feltesse, et al., The PDF $4 L H C$ Working Group Interim Recommendations, arXiv:1101.0538.

[128] T. Gleisberg et al., Event Generation With SHERPA 1.1, JHEP 10 (2009) 007, arXiv:0811.4622.

[129] S. Catani, F. Krauss, R. Kuhn, y B. Webber, QCD matrix elements + parton showers, JHEP 0111 (2001) 063, arXiv:hep-ph/0109231. 
[130] F. Krauss, Matrix elements and parton showers in hadronic interactions, JHEP 0208 (2002) 015, arXiv: hep-ph/0205283.

[131] C. Anastasiou, L. J. Dixon, K. Melnikov, y F. Petriello, High precision QCD at hadron colliders: Electroweak gauge boson rapidity distributions at NNLO, Phys. Rev. D69 (2004) 094008 , arXiv: hep-ph/0312266.

[132] J. Alwall et al., MadGraph/MadEvent v4: The New Web Generation, JHEP 09 (2007) 028, arXiv: 0706.2334

[133] T. Sjostrand, S. Mrenna, y P. Skands, PYTHIA 6.4 physics and manual, JHEP 05 (2006) 026, arXiv: hep-ph/0603175.

[134] P. Golonka y Z. Was, PHOTOS Monte Carlo: A Precision tool for QED corrections in $Z$ and $W$ decays, Eur.Phys.J. C45 (2006) 97-107, arXiv:hep-ph/0506026.

[135] N. Davidson, G. Nanava, T. Przedzinski, E. Richter-Was, y Z. Was, Universal Interface of TAUOLA Technical and Physics Documentation, Comput.Phys.Commun. 183 (2012) 821-843, arXiv: 1002.0543 .

[136] K. Melnikov, M. Schulze, y A. Scharf, QCD corrections to top quark pair production in association with a photon at hadron colliders, Phys.Rev. D83 (2011) 074013, arXiv:1102.1967.

[137] H. Group, Search for ttH production in the diphoton channel with $20.3 \mathrm{fb}^{-1}$ of data at $8 \mathrm{TeV}$, Tech. Rep. ATL-COM-PHYS-2013-866, CERN, Geneva, Jun, 2013.

[138] P. Nason, A New method for combining NLO QCD with shower Monte Carlo algorithms, JHEP 0411 (2004) 040, arXiv: hep-ph/0409146.

[139] S. Frixione, P. Nason, y C. Oleari, Matching NLO QCD computations with Parton Shower simulations: the POWHEG method, JHEP 0711 (2007) 070, arXiv:0709. 2092.

[140] S. Alioli, P. Nason, C. Oleari, y E. Re, A general framework for implementing NLO calculations in shower Monte Carlo programs: the POWHEG BOX, JHEP 1006 (2010) 043, arXiv: 1002 . 2581.

[141] W. Kilian, T. Ohl, y J. Reuter, WHIZARD: Simulating Multi-Particle Processes at LHC and $I L C$, Eur.Phys.J. C71 (2011) 1742, arXiv:0708.4233.

[142] M. Moretti, T. Ohl, y J. Reuter, O'Mega: An Optimizing matrix element generator, arXiv: hep-ph/0102195.

[143] N. Kidonakis, Two-loop soft anomalous dimensions for single top quark associated production with a W- or H-, Phys.Rev. D82 (2010) 054018, arXiv: 1005.4451.

[144] J. M. Campbell, R. K. Ellis, y C. Williams, Vector boson pair production at the LHC, JHEP 1107 (2011) 018, arXiv:1105.0020.

[145] M. H. Seymour y M. Marx, Monte Carlo Event Generators, in Proceedings, 69th Scottish Universities Summer School in Physics : LHC Phenomenology (SUSSP69), pp. 287-319. 2013. arXiv:1304.6677. http://inspirehep.net/record/1229804/files/arXiv: 1304.6677.pdf. 
[146] ATLAS Collaboration, Performance of the Electron and Photon Trigger in pp Collisions at $\sqrt{s}=7 \mathrm{TeV}$, Tech. Rep. ATLAS-CONF-2011-114, CERN, Geneva, Aug, 2011.

https://cds. cern. ch/record/1375551.

[147] D. Damazio, T. Kono, F. Monticelli, y G. Pasztor, Performance of the ATLAS Electron and Photon Triggers in pp Collisions at $\sqrt{s}=8 \mathrm{TeV}$ in 2012, Tech. Rep. ATL-COM-DAQ-2013-121, CERN, Geneva, Oct, 2013.

[148] M. Guchait y D. Sengupta, Event-shape selection cuts for supersymmetry searches at the LHC with $7 \mathrm{TeV}$ energy, Phys. Rev. D 84 (2011) 055010.

http://link.aps.org/doi/10.1103/PhysRevD.84.055010.

[149] A. Bocci, L. Shi, y Z. Liang, Measurements of the $e \rightarrow \gamma$ fake rate, Tech. Rep. ATL-COM-PHYS-2014-026, CERN, Geneva, Jan, 2014. https://cds. cern. ch/record/1643300.

[150] A. Kuhl, J. Mitrevski, y J. Nielsen, Search for photon, $b \bar{b}$ and $E_{\mathrm{T}}^{\text {miss }}$ final state in $\sqrt{s}=8 \mathrm{TeV}$ pp collisions at the LHC using the ATLAS detector, Tech. Rep. ATL-COM-PHYS-2013-1379, CERN, Geneva, Oct, 2013.

[151] L. Moneta, K. Belasco, K. S. Cranmer, S. Kreiss, A. Lazzaro, et al., The RooStats Project, PoS ACAT2010 (2010) 057, arXiv: 1009.1003.

[152] ATLAS Collaboration, Improved luminosity determination in pp collisions at $\sqrt{s}=7 \mathrm{TeV}$ using the ATLAS detector at the LHC, The European Physical Journal C 73 no. 8, (2013) 1-39. http://dx.doi.org/10.1140/epjc/s10052-013-2518-3.

[153] S. van der Meer, Calibration of the effective beam height in the ISR, Tech. Rep. CERN-ISR-PO-68-31. ISR-PO-68-31, CERN, Geneva, 1968. https://cds. cern. ch/record/296752.

[154] https://twiki.cern.ch/twiki/bin/view/AtlasProtected/PhotonID2012.

[155] https://twiki.cern.ch/twiki/bin/viewauth/AtlasProtected/EfficiencyMeasurements.

[156] https://twiki.cern.ch/twiki/bin/view/AtlasProtected/EGammaCalibrationGE020.

[157] https://twiki.cern.ch/twiki/bin/viewauth/AtlasProtected/ MCPAnalysisGuidelinesData2012.

[158] https:

//twiki.cern.ch/twiki/bin/viewauth/AtlasProtected/JetEtmissRecommendations2012.

[159] http://atlantis.web.cern.ch/atlantis/.

[160] http://hepdata.cedar.ac.uk/view/ins 1383883.

[161] https://atlas.web.cern.ch/Atlas/GROUPS/PHYSICS/CombinedSummaryPlots/SUSY/.

[162] ATLAS Collaboration, Search for photonic signatures of gauge-mediated supersymmetry in $8 \mathrm{TeV}$ pp collisions with the ATLAS detector, Phys. Rev. D 92 no. 7, (2015) 072001, arXiv: 1507.05493. 